\title{
DESARROLLO DE PROCESOS PARA FORMACIÓN DE CONTACTOS CON LÁSER PARA MÓDULOS THIN FILM CIGS PARA APLICACIONES FOTOVOLTAICAS
}

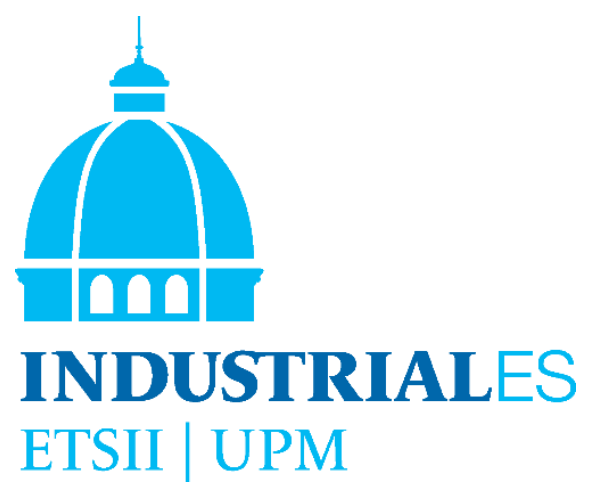

\author{
Yu Chen \\ Departamento de Física Aplicada a la Ingeniería Industrial \\ Universidad Politécnica de Madrid
}

Para la obtención de Grado de Doctor

Máster en Tecnología Láser

Noviembre 2016 
Título:

\title{
Desarrollo de procesos para formación de contactos con láser para módulos thin film
} CIGS para aplicaciones fotovoltaicas

\author{
Autor: \\ Yu Chen
}

Director de tesis:

Dr. Miguel Morales Furió

Tribunal nombrado por el Mgfco. y Excmo. Sr Rector de la Universidad Politécnica de Madrid el día 28 de octubre de 2016.

Tribunal:

\author{
Presidente: Carlos Luis Molpeceres Álvarez \\ Secretario: Sara Lauzurica Santiago \\ Vocal: Julio Cárabe López \\ Vocal: Elisa María Ruiz Navas \\ Vocal: Cristóbal Voz Sánchez \\ Suplente: Susana María Fernández Ruano \\ Suplente: Pablo Rafael Ortega Villasclaras
}

Realizado el acto de lectura y defensa de la tesis el día 14 de noviembre de 2016

Calificación de la Tesis

El presidente:

Los Vocales:

El Secretario: 


\section{AGRADECIMIENTOS}

En primer lugar, me gustaría mostrar mi mayor agradecimiento a Miguel Morales, el director de esta tesis, por su dedicación, su inestimable apoyo e infinita paciencia para encaminar este trabajo de investigación y carrera científica. También agradezco profundamente a Calos Molpeceres, el director del Centro Láser, por su ayuda que me brindó y por confianza depositada a la hora de diseñar los experimentos, aportando gran libertad. Sin ellos, este trabajo no hubiera sido posible.

Deseo expresar también mi agradecimiento al Departamento de Física Aplicada a la Ingeniería Industrial de la E.T.S.I.I de la Universidad Politécnica de Madrid y al Centro Láser U.P.M por la posibilidad de iniciar mi carrera doctorado y por los medios puestos a mi disposición para la realización de esta tesis.

Me gustaría dar las gracias a todas y cada una de los compañeros de Centro Láser que contribuyeron a que este trabajo fuera una realidad, en especial a David, Juanjo, Andrés, Jagdheesh, Alberto y a los que han seguido su carrera fuera del centro, Isabel, Manuel, Luis.

También quiero agradecer al grupo del Prof. Arnold de la Universidad de Princeton su colaboración para realizar los estudios de los regímenes de transferencia empleando técnicas de imagen de alta velocidad y al grupo de Micro y Nanotecnología de la Universidad Politécnica de Cataluña su colaboración en la caracterización de las células solares metalizadas mediante LIFT. Además, el trabajo está parcialmente financiado en el marco de los siguientes proyectos, Comisión Europea APPOLO FP7-2013-NMP-ICTFOF.609355 y MINECO proyectos SIMLASPV-MET (ENE2014-58454-R) y HELLO (ENE201348629 -C4-3-R).

Por último, me gustaría agradecer a mi familia por su aliento, comprensión y apoyo incondicional para que este trabajo saliera adelante.

Yu Chen

Madrid, agosto de 2016. 


\section{Contents}

List of Figures $\quad$ vi

$\begin{array}{lc}\text { List of Tables } & \text { xiv }\end{array}$

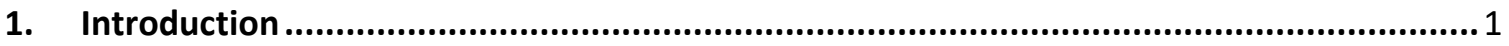

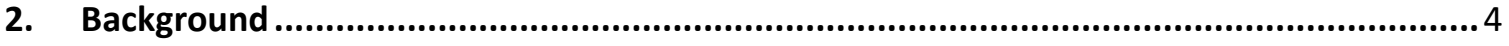

2.1 Influence of metallization process on efficiency of solar cell .................................4

2.2 Influence of metal contact geometry on power loss and series resistance ................9

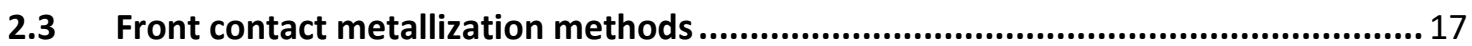

2.3.1 Photolithographically defined contacts.................................................. 19

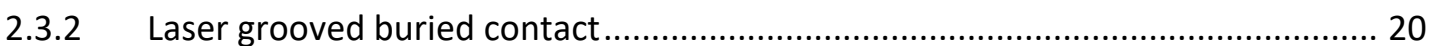

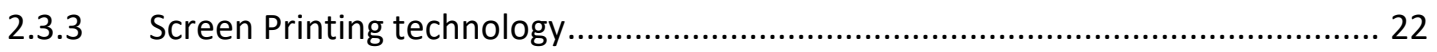

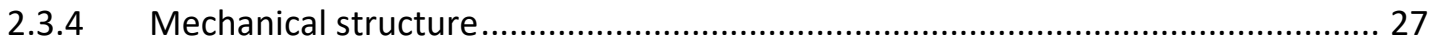

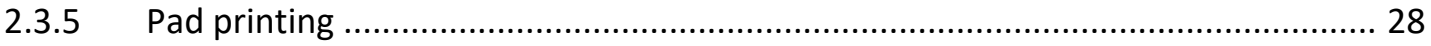

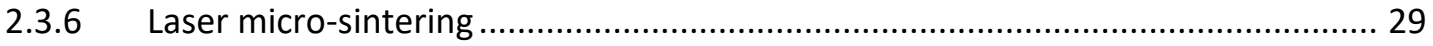

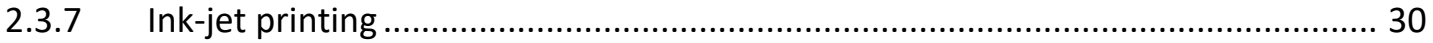

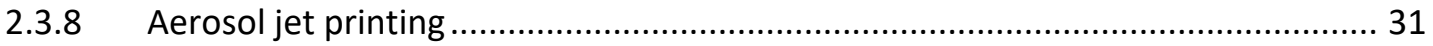

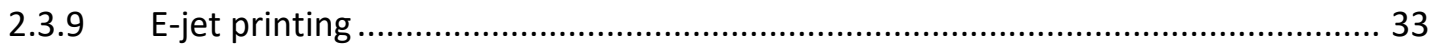

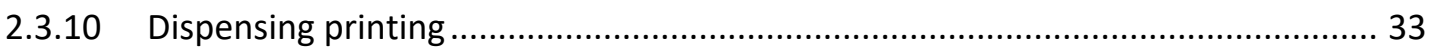

3. Laser-induced forward transfer (LIFT) ............................................................. 38

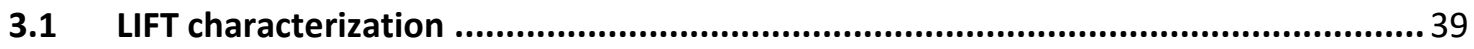

3.2 Different LIFT techniques...........................................................................40

3.2.1 LIFT of solid materials .................................................................... 43

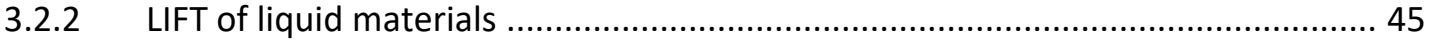

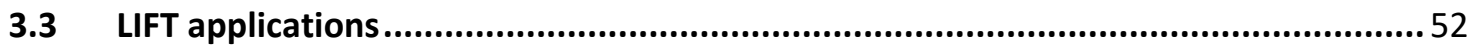

4. Experimental and characterization method .................................................... 56

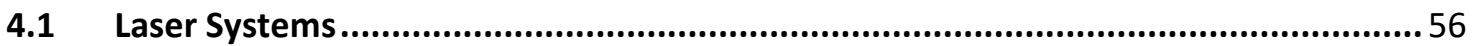

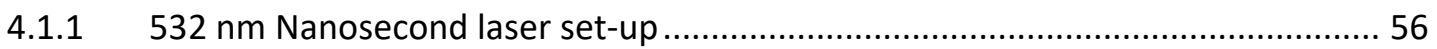

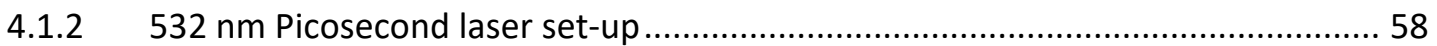

4.1.3 $1064 \mathrm{~nm}$ Picosecond laser set-up .......................................................... 59

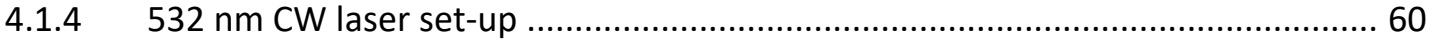

4.2 Beam waist Measurement...................................................................... 62

4.3 Donor preparation.................................................................................66 66 


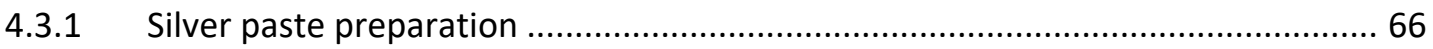

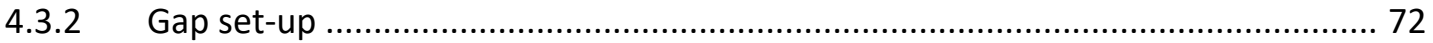

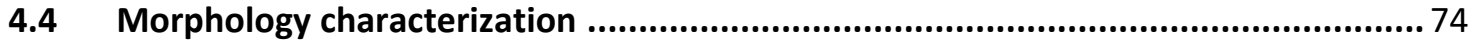

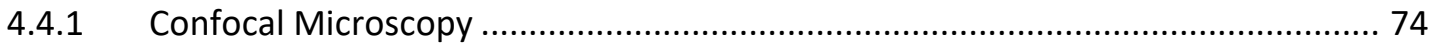

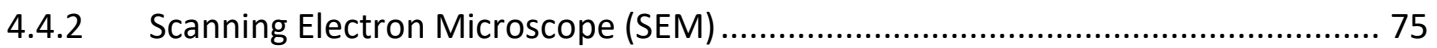

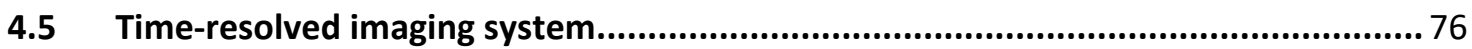

4.6 Electrical characterization method ........................................................................... 77

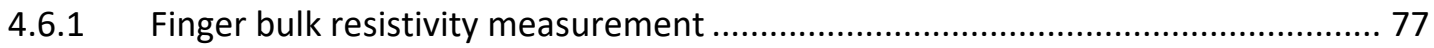

4.6.2 Busbar bulk resistivity measurement ................................................................. 80

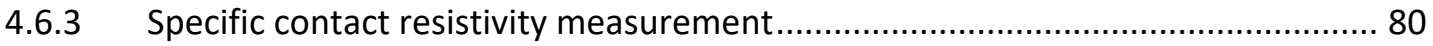

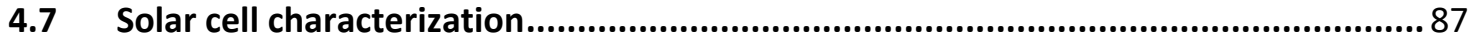

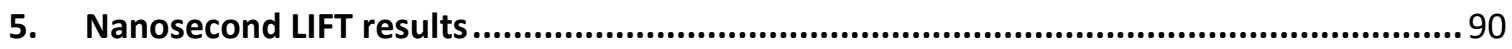

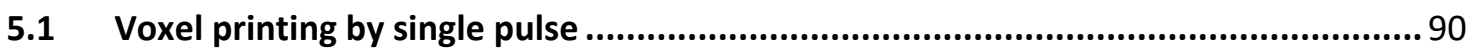

5.1.1 Morphological characterization of printed voxel ............................................... 90

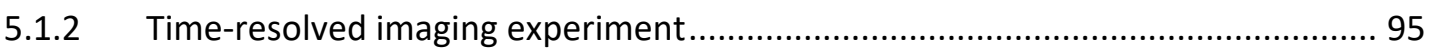

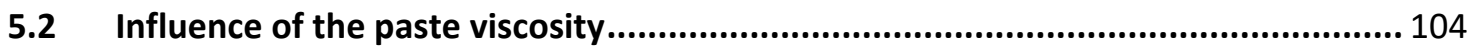

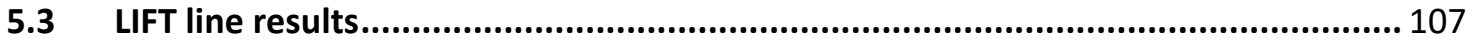

5.4 Influence of the wetting with different acceptor in LIFT ...................................... 111

5.5 Large area and design flexibility of LIFT process ................................................. 117

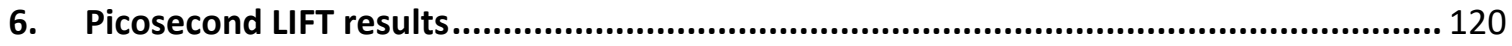

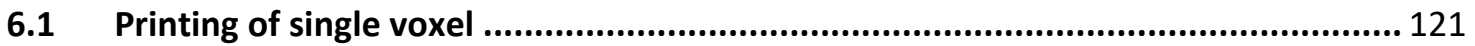

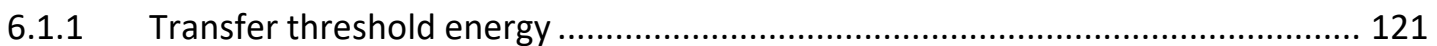

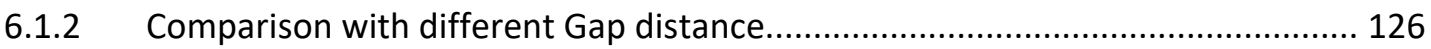

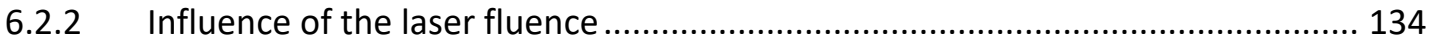

6.2.3 Lines drawn using polygon scanner ............................................................. 136

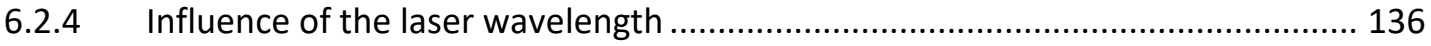

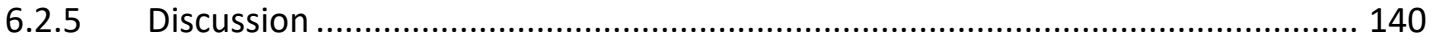

7. Laser curing/sintering/melting of the silver paste.......................................................... 146

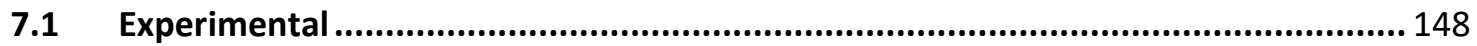

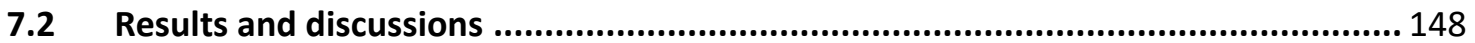

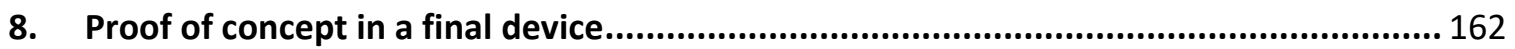

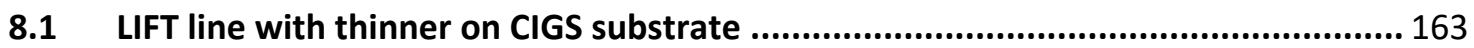

8.2 Mechanical properties of printed lines ................................................................ 165 


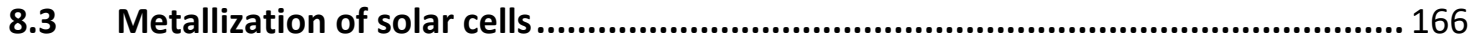

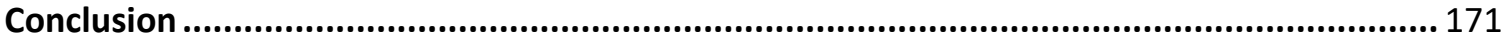

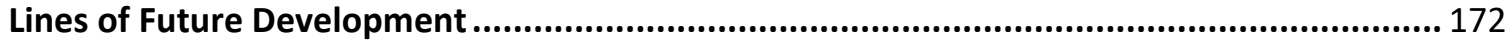

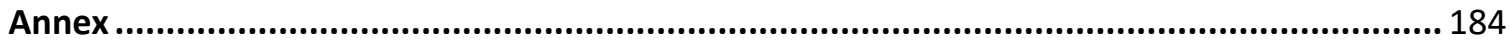

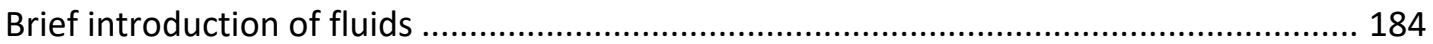




\section{List of Figures}

Figure 2.1: Solar cell circuit model with parasitic resistances. 5

Figure 2.2: Effect of series resistance on the current-voltage characteristic of a solar cell

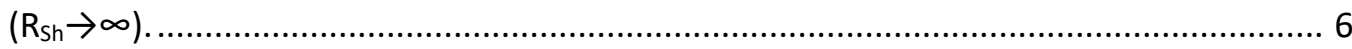

Figure 2.3: The general solar cell fill factor diagram. ...................................................... 8

Figure 2.4: Schematic diagram of series resistance distribution on solar cell. ....................... 9

Figure 2.5: Schematic diagram of a simple pattern of front contact. ................................ 10

Figure 2.6: Power loss fraction as a function of finger width in context of three different

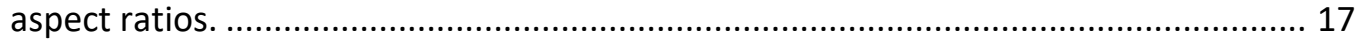

Figure 2.7: The classification of metallization method ..................................................... 19

Figure 2.8: Scheme of photolithography and metal evaporation process for the front side

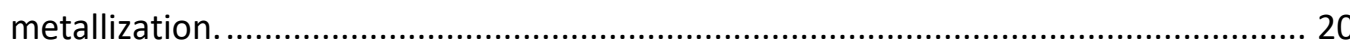

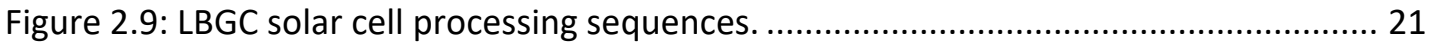

Figure 2.10: Schematic of laser grooved buried contact solar cell. ..................................... 21

Figure 2.11: Schematic of Screen Printing process. ........................................................... 22

Figure 2.12: SEM images of screen printing 360 mesh screen, $15 \mu \mathrm{m}$ wire diameter, $100 \mu \mathrm{m}$

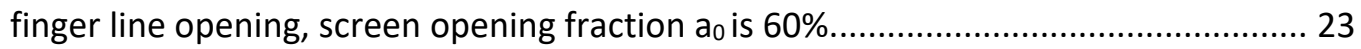

Figure 2.13: Comparisons between conventional SP (up) and DP (down) method: SEM cross section, 3D laser scan and electroluminescence images. ............................................ 25

Figure 2.14: Schematic of the light-induced plating process .............................................. 25

Figure 2.15: SEM image of the cross section of screen-printed contact by applying LIP

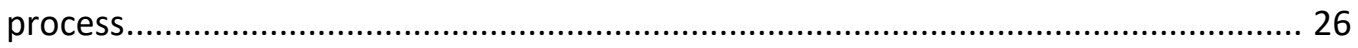

Figure 2.16: Schematic of the mechanical structure method using etching paste ............... 28

Figure 2.17: Schematic of the pad printing process ........................................................... 29

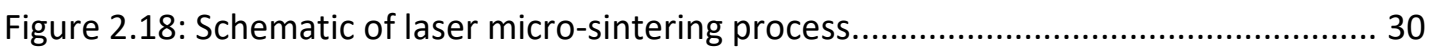

Figure 2.19: Schematic of inkjet printing system (a) drop-on-demand system with piezoelectric actuator (DOD) (b) continuous stream ink-jet system (CIJ). Adapted from

Figure 2.20: Schematic of the working principle of pneumatic and ultrasonic atomizer. .... 32

Figure 2.21: Schematic of principle of dispensing printing. ................................................ 33

Figure 2.22: Schematic of four principle nozzle-based printing techniques ....................... 34 
Figure 2.23: Cross sectional SEM image of the electrode printing by electrostatic-forceassisted dispensing printing technique with a high aspect ratio of $0.79 . . . . . . . . . . . . . . . . . . . .36$

Figure 3.1: Schematic of the traditional LIFT process and its working principle................... 38

Figure 3.2: Schematic diagram of the system used to capture high-speed video images .. 40

Figure 3.3: Working principle of Matrix-Assisted Pulsed Laser Evaporation Direct-Write (MAPLE-DW).

Figure 3.4: Working principle of laser molecular implantation (LMI) poly (butyl methacrylate, poly (ethyl methacrylate).

Figure 3.5: Scheme of blister-actuated laser-induced forward transfer process (BA-LIFT). . 42

Figure 3.6: Backscattered electron images of transferred thin tungsten pattern. The scaling bar indicates $100 \mu \mathrm{m}$. 44

Figure 3.7: High-aspect ratio examples of copper LIFT. a) $860 \mu \mathrm{m}$ long pillar close to the clearly visible edge of a microscope slide. b) Close-up of the pillar. Zooming in further shows that the pillar has a virtually homogeneous thickness: the center d) and top e) are around $4 \mu \mathrm{m}$ thick whereas the bottom diameter (e) is around $6 \mu \mathrm{m}$, due to drops landing next to the pillar

Figure 3.8: Time-resolved images of the hydrogel jet formation during LIFT at two laser fluence (a) $1.6 \mathrm{~J} / \mathrm{cm}^{2}$ and (b) $2.7 \mathrm{~J} / \mathrm{cm}^{2}$. 46

Figure 3.9: Scheme of the bubble dynamics and jet formation in LIFT process 47

Figure 3.10: Time-resolved images of liquid ejection into ambient air using (a)a 50-nm titanium absorbing layer and (b) a 7- $\mu \mathrm{m}$ polyimide absorbing layer

Figure 3.11: Time-resolved images of the hydrogel transfer on the acceptor during LIFT process with two laser influence (a) $1.6 \mathrm{~J} / \mathrm{cm}^{2}$ and (b) $2.7 \mathrm{~J} / \mathrm{cm}^{2}$. The gap distance between donor and acceptor is around $450 \mu \mathrm{m}$.....

Figure 3.12: SEM images of transferred silver nano-suspensions with different viscosities on Si acceptor substrate. (a, b) Transferred voxel with low viscosity silver nano-inks. (c, d) Transferred voxels with very high viscosity silver nanopastes. (e, f) Transferred voxels with high viscosity silver nanopastes

Figure 3.13: Still frame images obtained from the high-speed video (a) Evolution of the jet formation characteristic with low viscosity silver nano-inks. (b) Voxel transfer characteristic with very high viscosity silver nanopastes. (c) Voxel transfer characteristic with high viscosity silver nanopastes

Figure 4.1: Schematic Explore set-up for the LIFT process............................................... 57

Figure 4.2: Schematic of optical path for Ekspla Atlantic 355-60 laser set-up..................... 59

Figure 4.3: Schematic of CW 532nm laser system. 61 
Figure 4.4: Crater diameter represented as a function of laser pulse energy for $20 \mathrm{kHz}$ of repetition rate with the corresponding fitting line.

Figure 4.5: (Left) 2D and (right) 3D profile of (top) the IR beam in a pre-focus position, (bottom) VIS beam in a post-focus position. 65

Figure 4.6: Beam diameter measured with the beam profiler for the optical scanner. ........ 66

Figure 4.7: Micro-sized silver particles distributed on the CIGS substrate surface.

Figure 4.8: Silver paste viscosity showing the pseudo-plastic and thixotropic behavior. (Left) Viscosity vs time at different shear rate (Right) Equilibrium viscosity vs shear rate .... 70

Figure 4.9: Viscosity of silver paste with varying weight percentage of thinner (Left). Plateau viscosity of silver paste as a function of wt\% of thinner. 70

Figure 4.10: Different kinds of thixotropic behavior on startup of shear after various degrees of rest 71

Figure 4.11: Gap size set by Kapton ${ }^{\circledR}$ HN polyimide films used in the LIFT experiment. ...... 72

Figure 4.12: Deformation in the edges of the silver paste caused by the donor's own weight. 73

Figure 4.13: Gap set-up with Kapton ${ }^{\circledR}$ adhesive tape for silver paste donor 73

Figure 4.14: The Leica DCM3D Confocal Microscope used in the thesis. 74

Figure 4.15: The SEM Hitachi S-3000N work station. 75

Figure 4.16: Diagram of the high-speed time-resolved imaging system 76

Figure 4.17: Time-resolved images of transfers from a $10 \mu \mathrm{m}$ film of $\mathrm{N}$-methyl-2-pyrrolidone (NMP) by LIFT process 77

Figure 4.18: Four-point measurement of resistance between voltage sense connections 2 and 3. Current is supplied via force connections 1 and 4 . 78

Figure 4.19: Finger structure for bulk resistivity measurement. 78

Figure 4.20: Diagram of Four-point probe method by using KEITHLEY Model 2400 SourceMeter. 79

Figure 4.21: Diagram of bulk resistivity calculation method. 80

Figure 4.22: Diagram of busbar structure for bulk resistivity measurement. 80

Figure 4.23: Two contact resistance structure in cross section and top view. 83

Figure 4.24: Transfer length method test structure proposed by Shockley. 84

Figure 4.25: The TLM method with $\mathrm{R}_{\mathrm{T}}$ plotted as a function of uneven spacing. 85

Figure 4.26: Schematic of TLM structure with laser isolation. 86 
Figure 4.27: An example of IV curves characteristic calculation for solar cell

Figure 4.28: IV curves obtained by using a reference solar cell for the standard illumination calibration of the solar simulator in the laboratory.

Figure 4.29: Schematic of CIGS solar cell measurement with four probes 89

Figure 5.1: Schematic of the voxel printing matrix. 91

Figure 5.2: Microscope images of LIFT dots transferred using single shots of a ns laser with fluence varying from 0.6 to $5.9 \mathrm{~J} / \mathrm{cm}^{2}$ with two different donor thicknesses: (a) $30 \mu \mathrm{m}$ and (b) $50 \mu \mathrm{m}$ 92

Figure 5.3: Confocal microscope images of (a) donor films and (c) transferred dots from 1.3 $\mathrm{J} / \mathrm{cm}^{2}$ laser pulses with films of thickness (left) $30 \mu \mathrm{m}$ and (right) $50 \mu \mathrm{m}$. Profiles of both donor and dot are shown in (b) .....

Figure 5.4: Height, width and aspect ratio of the transferred dots as a function of laser peak fluence with respect to two different paste thicknesses $(30 \mu \mathrm{m}$ and $50 \mu \mathrm{m})$. 94

Figure 5.5: Time-resolved images of LIFT of silver paste from a donor film of thickness 120 $\mu \mathrm{m}$ using laser fluences of approximately (a) 8.5 and (b) $23 \mathrm{~J} / \mathrm{cm}^{2}$.

Figure 5.6: Time-resolved images of LIFT of silver paste from a film of thickness $60 \mu \mathrm{m}$ using laser fluences of approximately (a) 2.8 , (b) 4.2 , and (c) $8.5 \mathrm{~J} / \mathrm{cm}^{2}$ 98

Figure 5.7: Time-resolved images of LIFT of silver paste from a film of thickness $12 \mu \mathrm{m}$ using laser fluences of approximately (a) 0.6 , (b) 2.3 , and (b) $8.5 \mathrm{~J} / \mathrm{cm}^{2}$... 99

Figure 5.8: Time-resolved images of LIFT of silver paste with a gap of $140 \mu \mathrm{m}$ between donor and acceptor. The film thicknesses and laser fluences are (a) $35 \mu \mathrm{m}$ and 6.8 $\mathrm{J} / \mathrm{cm}^{2}$ and (b) $60 \mu \mathrm{m}$ and $8.4 \mathrm{~J} / \mathrm{cm}^{2}$.

Figure 5.9: Schematic of transfer mechanisms for varying laser peak fluences and donor thicknesses with a fixed gap distance: (a) blister formation, when the fluence is below the transfer threshold; (b) cluster-dot transfer, for fluences slightly above the threshold; (c) explosive transfer, when the peak fluence is much higher than the threshold; and (d) concrete transfer, when the donor is thick enough relative to the gap that the acceptor prevents fragmentation during transfer. The last row shows the final voxel obtained after removing the donor 102

Figure 5.10: Time-resolved images of LIFT of silver paste show the difference between the LIFT in free air and the LIFT with an acceptor substrate with a gap of $140 \mu \mathrm{m}$ between donor and acceptor. The film thicknesses and laser fluences are (a) $35 \mu \mathrm{m}$ and 8.5 $\mathrm{J} / \mathrm{cm}^{2}$ and (b) $60 \mu \mathrm{m}$ and $8.4 \mathrm{~J} / \mathrm{cm}^{2}$.

Figure 5.11: Microscope images of LIFT dots transferred using single shots of a ns laser with pulse energy of $3.2 \mu \mathrm{J}$ and donor thicknesses of $50 \mu \mathrm{m}$ (a) With $4.2 \%$ of thinner, (b) original paste. 104 
Figure 5.12: The voxel dimension represented by Height, width and aspect ratio of the transferred dots as a function of laser peak fluence with respect to original paste and the paste with $4.2 \%$ with film thickness of $50 \mu \mathrm{m}$. 105

Figure 5.13: Time-resolved images of silver paste with thinner added ( $4.2 \%$ by weight). The laser pulse energy is $70 \mu \mathrm{J}$, and the film thickness is about $60 \mu \mathrm{m}$. 106

Figure 5.14: LIFT lines of silver paste on a texturized c-Si wafer and its donor substrate. obtained with these process parameters: Paste thickness: $80 \mu \mathrm{m}$; Gap: $50 \mu \mathrm{m}$; pulse energy: $18 \mu \mathrm{J}$; Frequency: $20 \mathrm{kHz}$; Speed: $2.0 \mathrm{~m} / \mathrm{s}$.

Figure 5.15: Confocal images of the LIFT lines obtained with these process parameters:

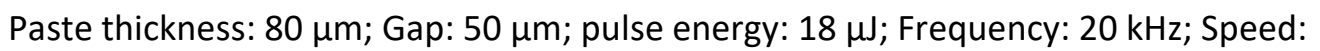
$2.0 \mathrm{~m} / \mathrm{s}$. 108

Figure 5.16: LIFT lines of silver paste on a texturized c-Si wafer obtained with these process parameters: Paste thickness: $80 \mu \mathrm{m}$; Gap: $50 \mu \mathrm{m}$; pulse energy: $18 \mu \mathrm{J}$; Frequency: 20 $\mathrm{kHz}$; Speed: $2.0 \mathrm{~m} / \mathrm{s}$.

Figure 5.17: Confocal images of the LIFT lines obtained with these process parameters: Paste thickness: $80 \mu \mathrm{m}$; Gap: $50 \mu \mathrm{m}$; pulse energy: $18 \mu \mathrm{J}$; Frequency: $20 \mathrm{kHz}$; Speed: $2.0 \mathrm{~m} / \mathrm{s}$.

Figure 5.18: Different LIFT lines confocal and SEM profiles obtained with these process parameters: Paste thickness: $80 \mu \mathrm{m}$; Gap: $50 \mu \mathrm{m}$; pulse energy: $20 \mu \mathrm{J}$; Frequency: 20 $\mathrm{kHz}$; Speed: $2.0 \mathrm{~m} / \mathrm{s}$.

Figure 5.19: SEM images of LIFT lines confocal obtained the optimal process parameters: Paste thickness: $80 \mu \mathrm{m}$; Gap: $50 \mu \mathrm{m}$; pulse energy:20 $\mu$ j; Frequency: $20 \mathrm{kHz}$; Speed: $2.0 \mathrm{~m} / \mathrm{s}$.

Figure 5.20: Height, width and aspect ratio of the transferred dots with different substrate (Polished c-Si, Textured c-Si and TCO film of CIGS) as a function of laser peak fluence with respect to two different paste thicknesses $(30 \mu \mathrm{m}$ and $50 \mu \mathrm{m})$.

Figure 5.21: Confocal images of the voxel and line printed on polished c-Si substrate with laser pulse energy of $2.7 \mu \mathrm{J}$ : (a) voxel, (b) line.

Figure 5.22: Confocal images of the voxel and line printed on CIGS substrate with laser pulse energy of $2.7 \mu \mathrm{J}$ : (a) voxel, (b) line.

Figure 5.23: Confocal images of the voxel and line printed on textured c-Si substrate with laser pulse energy of $2.7 \mu \mathrm{J}$ : (a) voxel, (b) line.

Figure 5.24: SEM micrographs of cross-section of lines printed using the same experimental conditions onto different substrates: (a) polished c-Si (b) TCO film on $\mathrm{CIGS}$, (c) textured c-Si. The contact angles between the paste and the substrate surface are respectively $20^{\circ}, 30^{\circ}$, and $75^{\circ}$.

Figure 5.25: Front grid printed with LIFT on a c-Si cell. 
Figure 5.26: Some freeform patterns printed with LIFT on texture c-Si cell.

Figure 6.1: The schematic of voxel printing with ps-pulsed laser. The laser pulse energy from $8.3 \mu \mathrm{J}$ to $57.3 \mu \mathrm{J}$ (a) width of the hole, (b) depth of the hole, (c) width of the voxel, (d) height of the voxel.

Figure 6.2: The dimension of the hole left in the donor and the voxel deposited on the acceptor as a function of laser pulse energy from $8.3 \mu \mathrm{J}$ to $57.3 \mu \mathrm{J}$ (a) width of the hole, (b) depth of the hole, (c) width of the voxel, (d) height of the voxel.

Figure 6.3: The dimension of the hole left in the donor with pulse energy varying from 0.7 $\mu \mathrm{J}$ to $8.3 \mu \mathrm{J}$.

Figure 6.4: The width of the hole left in the donor with pulse energy of 5.6 (left) and 6.9 (right) $\mu \mathrm{J}$. 125

Figure 6.5: Microscope images of picosecond LIFT silver paste with different burst counts:

(a) 10, (b) 50, (c) 500 . 126

Figure 6.6: Microscope images of LIFT dots transferred using single shots of ps laser and with fluence varying from 8.3 to $57.3 \mu \mathrm{J}$ with two different gap distance: (a) $50 \mu \mathrm{m}$ and (b) $100 \mu \mathrm{m}$.

Figure 6.7: Height, width and aspect ratio of the transferred dots as a function of fluence varying from 0.7 to $57.3 \mu \mathrm{J}$ with respect to two different gap distance (50 $\mu \mathrm{m}$ and 100 $\mu \mathrm{m})$.

Figure 6.8: The dimension of the hole left in the donor and the voxel deposited on the acceptor with GAP distance of $50 \mu \mathrm{m}$.

Figure 6.9: The morphology of the best voxel using a donor film thickness of $50 \mu \mathrm{m}, 50 \mu \mathrm{m}$ gap distance and pulse energy threshold of $8.3 \mu \mathrm{J}$.

Figure 6.10: Confocal images of the middle part of (d) transferred line and (b) donor films with $8.3 \mu \mathrm{J}$ laser pulses, $35 \mu \mathrm{m}$ film thickness, $50 \mu \mathrm{m}$ gap distance and different pitch distances: $150 \mu \mathrm{m}, 100 \mu \mathrm{m}, 80 \mu \mathrm{m}$ and $60 \mu \mathrm{m}$ (from left to right). Laser pulse and cross-sectional profile of both holes left in the donor and the transferred pastes are shown in (a) and (c).

Figure 6.11: Confocal images of the beginning part of the line (a) Laser pulse (b) donor and (d) transferred line and (c) profiles in pairs of donor and acceptor with $8.3 \mu \mathrm{J}$ pulse energy with different pitch distances: $150 \mu \mathrm{m}, 100 \mu \mathrm{m}, 60 \mu \mathrm{m}$ and $30 \mu \mathrm{m}$ (from left to right). Laser beam scanning direction is from right to left.

Figure 6.12: Confocal images of the middle part of the line (a) Laser pulse (b) donor and (d) transferred line and (c) profiles of both holes left in donor and transferred line on the acceptor with pitch distance of $100 \mu \mathrm{m}$ and different laser fluence: 5.6, 8.3, 9.7 and $11.2 \mu \mathrm{J}$ (from left to right)...... 
Figure 6.13: Cross-section profile of the printed line with $40 \mu \mathrm{m}$ film thickness, $50 \mu \mathrm{m}$ gap distance and laser fluence of $11.2 \mu \mathrm{J}$.

Figure 6.14: Microscope images of LIFT line transferred with different pulse energies by using polygon scanner. 136

Figure 6.15: Microscope images of LIFT line transferred using $1064 \mu \mathrm{m}$ ps laser with $8.8 \mu \mathrm{J}$ pulse energy with varied pitch distances from 2 to $200 \mu \mathrm{m}$.

Figure 6.16: Microscope and Confocal images of the middle part of the transferred line on the acceptor with pitch distance of $100 \mu \mathrm{m}$ and different laser fluence: 5.8, 8.8, 9.9 and $11.2 \mu \mathrm{J}$ (from left to right).

Figure 6.17: Microscope and Confocal images of the middle part of the transferred line on the acceptor with pitch distance of 100 . 139

Figure 6.18: Schematic of the laser pulse overlapping effect with short pitch distance on the donor substrate in the LIFT process.

Figure 6.19: Top view microscope images through the glass of the middle parts of laser scanning trace with pitch distance of $100 \mu \mathrm{m}, 80 \mu \mathrm{m}, 60 \mu \mathrm{m}$ and $30 \mu \mathrm{m}$ (from left to right).

Figure 6.20: False-color confocal images of LIFT lines printed using (a) ns- and (b) ps-laser (c) cross-sectional profiles of the lines.

Figure 7.1: Schematic diagram showing sintering processing parameters

Figure 7.2: SEM image of the surface of as-deposited line silver paste without laser treatment.

Figure 7.3: SEM images of the sintered region on silver paste surface with laser power of $2.7 \mathrm{~W}$ and varied speed: (a) $40 \mathrm{~mm} / \mathrm{s}$, (b) $50 \mathrm{~mm} / \mathrm{s}$, (c) $60 \mathrm{~mm} / \mathrm{s}$, (d) $70 \mathrm{~mm} / \mathrm{s}$, (e) 80

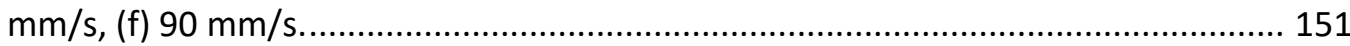

Figure 7.4: Width of the molten zone vs scan speed at laser power of $2.7 \mathrm{~W}$..................... 151

Figure 7.5: SEM images of the sintered region on silver paste surface with fixed scan speed of $40 \mathrm{~mm} / \mathrm{s}$ and varied laser power: (a) $2.7 \mathrm{~W}$, (b) $3.1 \mathrm{~W}$, (c) $3.5 \mathrm{~W}$, (d) $3.9 \mathrm{~W}$, (e) $4.4 \mathrm{~W}$, (f) $4.7 \mathrm{~W}$.

Figure 7.6: Influence of laser power on the width of the molten zone of silver particles at scan speed of $40 \mathrm{~mm} / \mathrm{s}$.

Figure 7.7: Influence of energy per unit length on the width of the molten zone of silver particles.

Figure 7.8: SEM images of the sintered region on silver paste surface with scan speed of 4 $\mathrm{mm} / \mathrm{s}$ and two different laser power: (a) $3.1 \mathrm{~W}$, (b) $5.8 \mathrm{~W}$. 
Figure 7.9: SEM images of the sintered region on silver paste surface with scan speed of $4 \mathrm{~mm} / \mathrm{s}$, laser power of $2.7 \mathrm{~W}$ and two scan path numbers: (a) $\mathrm{N}=1$, (b) $\mathrm{N}=5$. 155

Figure 7.10: Processing window for single path scan on silver paste surface. Processing conditions are scan speed $(4-90 \mathrm{~mm} / \mathrm{s})$, laser power $(2.7-5.8 \mathrm{~W})$ and scan spacing of $20 \mu \mathrm{m}$.

Figure 7.11: SEM images of the sintered region on silver paste surface with two-step treatment, first preheating with scan speed of $70 \mathrm{~mm} / \mathrm{s}$, laser power of $1.5 \mathrm{~W}$; second scan path with scan speed of $10 \mathrm{~mm} / \mathrm{s}$ and different laser power: (a) $3.1 \mathrm{~W}$, (b) $1.9 \mathrm{~W}$, (c) $1.2 \mathrm{~W}$, (d) $0.6 \mathrm{~W}$. 158

Figure 7.12: The bulk resistivity of finger line as a function of laser power with two-step sintering.

Figure 8.1: The confocal images of LIFT line shows good continuity but bad morphology with process parameters: pulse energy $(11 \mu \mathrm{J})$, scan speed $(1800 \mathrm{~mm} / \mathrm{s})$, laser frequency $(20 \mathrm{kHz})$, film thickness $(55 \mu \mathrm{m})$.

Figure 8.2: The confocal images of LIFT line shows good morphology but bad continuity with process parameters: pulse energy $(11 \mu \mathrm{J})$, scan speed $(1800 \mathrm{~mm} / \mathrm{s})$, laser frequency $(20 \mathrm{kHz})$, film thickness $(55 \mu \mathrm{m})$; (a) The line deposited by LIFT on the CIGS substrate, (b) Morphology of the printed line, (c) The morphology of the groove left in the donor.

Figure 8.3: The confocal images of LIFT line obtained by salver paste with $4.2 \mathrm{wt} \%$ of thinner. Process parameters: pulse energy $(11 \mu \mathrm{J})$, scan speed $(1800 \mathrm{~mm} / \mathrm{s})$, laser frequency $(20 \mathrm{kHz})$, film thickness $(55 \mu \mathrm{m})$; (a) Topography of the printed line in 3D vision, (b) Microscope image of the printed line, (c) The line deposited by LIFT on the CIGS substrate showing continuity, (d) The transverse profile of the line. 164

Figure 8.4: The transverse profiles of LIFT line obtained with optimal process parameters of the ns-pulse green laser on both textured c-Si and CIGS substrates......................... 165

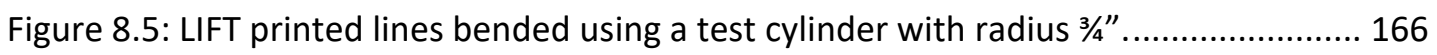

Figure 8.6: Schematic of front contact design in the final device. ................................... 166

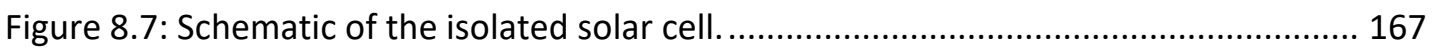

Figure 8.8: I-V curve of the CIGS solar cell sintered in the furnace ................................... 168

Figure 8.9: I-V curve of the CIGS solar cell sintered with CW laser step by step and finally in the furnace 


\section{List of Tables}

Table 2.1: Material and solar cell operating properties used in the grid geometric analysis

Table 2.2: Comparisons of geometrical and electrical parameters of the finger defined by screen-printed (SP), photolithographically defined contacts and laser grooved buried contact (LGBC).

Table 2.3: Comparison of four nozzle-based printing techniques applied metallization on solar cell.

Table 2.4: Comparisons of different front contact metallization techniques.

Table 3.1: Laser-induced forward transferred liquid material and parameters used for the studies.

Table 4.1: Explore laser performance specifications for $532 \mathrm{~nm}$ of wavelength. 56

Table 4.2: Ekspla Atlantic laser performance specifications. 58

Table 4.3: Onefive Katana-10 HP laser performance specifications for $1064 \mathrm{~nm}$ of wavelength. 60

Table 4.4: Millennia Pro s laser output characteristics. 60

Table 4.5: Experimental set-up used in the thesis. 62

Table 4.6: DuPont Solamet ${ }^{\circledR}$ PV17F properties 68

Table 4.7: The combination of three Kapton ${ }^{\circledR}$ film thicknesses; ${ }^{*}$ Repeated combination.. 72

Table 5.1: LIFT processing parameters of voxel printing on different substrate

Table 6.1: The dimension of the hole left in the donor and the deposit on the acceptor with varying pulse energy.

Table 6.2: The dimension of the hole left in the donor and the deposit on the acceptor with pulse energy of $6.9 \mu \mathrm{J}$

Table 6.3: The dimension of the hole left in the donor and the deposit on the acceptor with

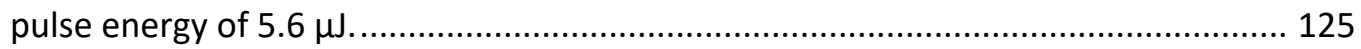

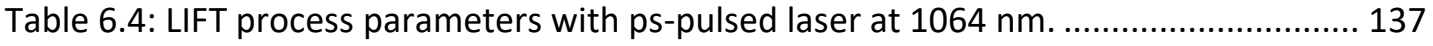

Table 6.5: Comparison of ns- and ps- LIFT process parameters and line results. 144

Table 7.1: The different temperature and process effect in the curing, sintering and melting.

Table 7.2: Process parameters for selective laser curing/sintering. 150

Table 7.3: The electrical properties of the laser treated lines. 159 
Table 7.4: The resistivity of the silver paste lines sintered using different method.

Table 8.1: Process parameters and geometry of the front contacts printed by LIFT on CIGS

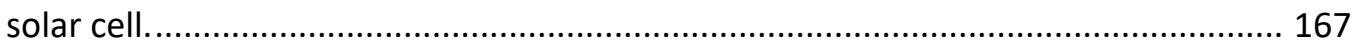

Table 8.2: CIGS solar cell characterization results with solar simulator. ............................ 168

Table 8.3: Process parameters and geometry of the front contacts printed by LIFT on CIGS

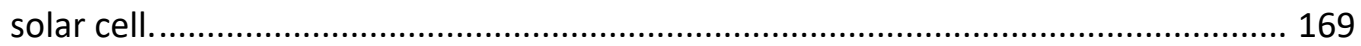

Table 8.4: CIGS solar cell characterization results with solar simulator. ............................ 170 


\section{Resumen}

La metalización es un paso clave en la fabricación de células solares, además de ser el paso con mayor impacto en el precio final de la célula. Entre las técnicas de metalización usadas en la fabricación de células solares fotovoltaicas, la serigrafía es el método más ampliamente empleado. Es un sistema robusto y económico para la impresión de los dedos conductores en la industria fotovoltaica. Sin embargo, debido a las limitaciones de la técnica, difícilmente puede imprimir líneas de pasta de plata con altura de más de 30 micras. Por lo tanto, la relación de aspecto de línea (cociente entre altura y anchura) se limita a menos de 0,4. En los últimos años, la investigación en el tema se ha centrado en el desarrollo de técnicas de metalización innovadoras y rentables para la impresión de patrones de alta resolución.

Durante las últimas décadas, las tecnologías de fabricación basadas en láser se han convertido en una herramienta esencial en la industria fotovoltaica. Entre estas técnicas, el corte por láser y el aislamiento de borde han sido ampliamente utilizados en las células solares basadas en silicio cristalino. Y en el caso de las células basadas en lámina delgada (silicio amorfo, CIGS, ...) el láser se ha empleado para realizar la interconexión monolítica de las mismas. Con el desarrollo de nuevas tecnologías y sistemas láser, los investigadores están permitiendo que las nuevas técnicas empleadas en el laboratorio para producir células solares de alta eficiencia se encuentren cada vez más maduras para su aplicación industrial.

En la presente Tesis, se propone un nuevo concepto de metalización láser de células solares en dos pasos. En un primer paso se imprime el patrón de líneas de pasta de plata, para ello se ha adaptado la técnica de transferencia de material inducida por láser (Laserinduced Forward Transfer, LIFT). El LIFT es una técnica de escritura directa con láser empleada en otras aplicaciones y que permite imprimir con gran precisión pequeños volúmenes de material. Para poder definir los contactos frontales metálicos en 
dispositivos fotovoltaicos rígidos o flexibles (en geometría plana) es necesario desarrollar esta técnica para imprimir en áreas grandes. En el segundo paso la línea depositada mediante LIFT es curada/sinterizada mediante láser. En este proceso al calentar la pasta en primer lugar se logra la eliminación del material orgánico, permitiendo que los granos de plata entren en contacto entre si, y al incrementarse más la temperatura se produce la fusión parcial de los mismos. Mediante este proceso la conductividad de la línea aumenta, y se obtienen conductividades similares a las logradas en procesos tradicionales de horno. El empleo del láser hace que el calentamiento sea local sin dañar a la célula, por lo que se puede definir como un paso a baja temperatura.

El estudio de los procesos LIFT con pastas de plata para la formación de contactos frontales, se ha realizado investigado desde la impresión de pequeños volúmenes (vóxel, del inglés volumetric pixel) con un solo pulso a la impresión de líneas mediante solapamiento de vóxeles.

Mediante una colaboración con la Universidad de Princeton ha sido posible observar, mediante técnicas de imagen de alta velocidad, cuatro regímenes de transferencia de pasta de plata (con granos de tamaño micrométrico) en el proceso de LIFT. En particular se ha observado un nuevo mecanismo de transferencia, que es totalmente diferente de los observados en estudios previos usando fluidos newtonianos o pastas con nanopartículas. En este proceso de transferencia se forma un puente de pasta estable entre el substrato donante y el substrato receptor, permitiendo la transferencia de un vóxel bien definido y con mayor relación de aspecto.

El estudio se amplía superponiendo vóxeles (empleando ejes lineales o un escáner galvanométrico) para imprimir líneas de pasta de plata con una alta relación de aspecto. Al analizar el proceso de impresión de líneas se observa la existencia de un valor umbral en el solapamiento de pulso por debajo del cual no se logra la transferencia debido a que el gas escapa por el hueco que va dejando la pasta al ser transferida y, por lo tanto, la presión aplicada se reduce. 
El proceso se ha desarrollado y optimizado para diferentes sustratos aceptores (obleas de silicio cristalino y $\mathrm{CIGS}$ sobre substratos flexibles) utilizando tantas fuentes láser en ns y ps, mostrando las ventajas y limitaciones de la técnica. Se han obtenido buenos resultados morfológicos en ambos sustratos, logrando imprimir líneas de pasta de plata con alturas de más de 60 micras y una relación de aspecto de más de 0,6.

Además de la influencia de los parámetros del proceso LIFT, la mojabilidad de la pasta de plata en el sustrato receptor juega un papel importante en la formación de líneas de alta resolución. Se ha observado que al aumentar la rugosidad del substrato se mejora la relación de aspecto de la línea transferida. Por lo que se propone en la presente Tesis la posibilidad de texturizar la superficie para mejorar la relación de aspecto de las líneas impresas.

Las líneas depositadas mediante LIFT y curadas/sinterizadas con len sustratos CIGS han mostrado una buena adherencia mecánica sometidas a ensayos de flexión y unas propiedades eléctricas mediante ensayo TLM semejantes a las obtenidas por procedimientos estándar.

Además, el estudio sistemático del proceso LIFT, ha permitido mostrar su capacidad para la impresión de cualquier patrón en áreas grandes, siendo una técnica adecuada tanto en el ámbito de los dispositivos fotovoltaicos como en dispositivos optoelectrónicos. Se ha demostrado la viabilidad del proceso realizando la metalización completa de una célula CIGS obteniendo una eficiencia superior al $11 \%$.

La presente Tesis propone un método completamente original de metalización basado en LIFT que supera algunas de las limitaciones encontradas en la serigrafía. La posibilidad de imprimir cualquier patrón en un área grande y a alta velocidad, su gran versatilidad para transferir todo tipo de materiales (tintas metálicas, nano-pastas y pastas) a transferir y sobre todo tipo de sustratos (rígidos o flexibles) hacen que esta técnica sea un buen 
candidato para su uso industrial en la fabricación de dispositivos fotovoltaicos u optoelectrónicos.

Este trabajo ha sido parcialmente financiado en el marco de los siguientes proyectos, Comisión Europea APPOLO FP7-2013-NMP-ICT-FOF.609355 y MINECO proyectos SIMLASPV-MET (ENE2014-58454-R) y HELLO (ENE2013-48629 -C4-3-R). 


\begin{abstract}
Metallization is one of the key and most expensive step in the typical solar cell fabrication. Amongst the metallization techniques of photovoltaic solar cell, the screen printing technique has achieved a great success. It is the most widely employed and robust method for finger printing in the present photovoltaic industry. However, due to the limitation of the technique, it can hardly print silver paste line with height more than 30 $\mu \mathrm{m}$. Therefore, the line aspect ratio is limited to less than 0.4. In the last few years, researchers have been focused on the development of innovative and cost-effective metallization techniques for printing high-resolution patterns.

During the last few decades, laser-based manufacturing technology has become an essential tool in the photovoltaic industrial. Among these techniques, laser cutting and edge isolation have already been widely used in the crystalline solar cell industrial manufacturing. And the laser scribing is also applied in the thin film technology. With the development in new laser technologies and system, public groups are bridging the gap between these laser techniques and high efficiency solar cells.

In this thesis work, a new concept of two-step front metallization of the solar cell is proposed aiming at adapting the Laser-induced forward transfer techniques (LIFT), a wellknown laser direct writing technique for material transfer towards 3D printing, to define the front metallic contacts onto flexible photovoltaic devices (specially in planar geometry) as the pre-metallization process. Combining another laser-based post-metallization technique, which is the selective laser curing/sintering, the deposited silver paste line can be functionalized in the direct write way by means of laser irradiation.
\end{abstract}

In the context, this Thesis proposes a completely original method, demonstrating the possibility of the formation of silver paste front contact lines with height more than 60 $\mu \mathrm{m}$ and an aspect ratio more than 0.6 by means of laser-induced forward transfer. This 
result obtained for the first time at international level in this work, breaking thought the limitation of the screen printing technique. The easy set-up and free-style pattern printing ability has revealed the feasibility for the industrial development of this technique in the future.

In this work LIFT for front contact formation process, investigated from voxel to line printing, has been developed and optimized for different acceptor substrates, demonstrating the advantages and limitations of the technique using both ns and pspulsed laser sources. Good results have been obtained in both crystalline silicon wafer and flexible CIGS thin film substrate. The lines deposited by LIFT and post-metallized by SLS in CIGS substrates has shown good mechanical adhesion under bending test and electrical properties under TLM test.

In particular, four transfer regimes of micro-sized silver paste are defined in the LIFT process. And among them a new transfer mechanism, which is totally different from those previously studies using Newtonian fluid or nanopastes, is observed and studied for the first time. This founding is highly remarkable for that the formation of silver paste bridge between donor and acceptor substrate leads to the concrete-dot voxel. By using computer controlled translation stages or galvanometric scanning mirrors, silver paste line with high aspect ratio can be formed by the overlapping of the voxel as the front contact on the photovoltaic substrate.

Moreover, in the line printing section it is found that the pitch distance for overlapping the voxels in line scanning condition exists a threshold value above which the transfer will be ceased due to the escaped pressure through the laser-induced tunnel. Apart from the influences of the LIFT process parameters, the interaction of silver paste and acceptor substrate through wetting and adhesion also play an important role in forming highresolution metal lines. From this point of view, an approach of increasing surface roughness by texturing is proposed in the thesis for the first time for preventing the spreading of the paste and keeping the line with high aspect ratio in the LIFT process. 
In addition, the work performed in the systematic study of LIFT process, which shows the high flexibility in material and acceptor substrate choosing and the ability for free-style pattern printing, is not only adapted to the scope of photovoltaic devices, but also is of highly relevance in the optoelectronics device toward 3D printing.

In the end, it is appreciated for a collaboration between Centro Laser of UPM, where the author made his work, and the department of mechanical and aerospace engineering of Princeton University, where most works of the time-resolved imaging experiment were made. This work was supported in the context of several projects with public funding, such as EUROPEAN COMISSION APPOLO FP7-2013-NMP-ICT-FOF.609355 and Spanish MINECO projects SIMLASPV-MET (ENE2014-58454-R) and HELLO (ENE2013-48629- C4-3R) and support from the NSF MRSEC program through the Princeton Center for Complex Materials (grant DMR-0819860) and the Rutgers-Princeton NSF IGERT on Nanotechnology for Clean Energy. 



\section{Introduction}

Metallization is regarded as one of the key but second most expensive step in the typical solar cell fabrication. Several different techniques [1-6] have been developed to overcome the limitations of the conventional screen-printing, which is the wellestablished and robust method to deposit metal contacts in solar cell industrial production. In this case, one of the aims of solar cell researchers and manufacturers is to find technologies leading to an increase in the efficiencies of solar cells while keeping costs low. Specifically, procedures capable of making better contacts by improving the aspect ratio and decreasing contact losses are sought.

Among those novel metallization techniques, LIFT performed with ns- or ps-pulses is a well-known technology to generate structured metallization onto substrates, but it has not yet been used to create the front contact fingers for a photovoltaics device in a single step, although a two-step approach has been developed recently . Laser-induced forward transfer (LIFT) is a direct-write laser technique, capable of transferring a variety of materials (especially metallic solid materials or material dissolved in an assisting matrix) in different sizes onto a number of substrates[7].

LIFT uses laser pulses to push thin disks of material from a donor film onto an acceptor substrate. The film coats a transparent substrate, and the laser beam is focused in the donor substrate/film interface. Throughout the duration of the pulse, the laser energy is deposited at the laser spot into the interface, vaporizing a small amount of the material and generating the expansion of the remaining material, accelerating the non-vaporized part of the donor film towards the acceptor substrate [8]. Attractive features of the LIFT process such as easy setup, high flexibility in choice of printed contents and costeffectiveness have even made an industrial implementation feasible [9]. It is thus possible to use LIFT for printing metallic contacts [10] and patterning solder paste for microelectronics [11] , or microwave interconnects in coplanar waveguides [12]. In those cases, inks or pastes with silver nanoparticles are used as donor materials. 
LIFT has been proved for the metallization of the front side of solar cells [13]. The feasibility of printing long, high-aspect-ratio lines of a commercial silver paste onto c-Si cells has been demonstrated in this work. The parametric window to achieve good results was narrow and sensitive to small changes in the parameters.

In order to control this printing process, it is desirable to understand the effects of the LIFT process parameters (laser pulse energy, silver paste donor thickness, and gap distance between donor and acceptor substrates) on the morphology of the transferred paste.

The effect of these experimental parameters on the mechanisms for material transfer using LIFT have been widely discussed in the case of solids [14, 15], Newtonian fluids [16, $17]$, and pastes $[12,18,19]$. However, the particular rheology, high viscosity, and particle size of the silver paste used strongly affect the transfer process and the shape and quality of the printed voxel, distinguishing its mechanisms from those observed previously. This thesis investigates mainly on the LIFT process of high viscosity micro-sized silver paste, which consists of micron-sized particles and is normally used for screen printing and offer an alternative method to the metallization of front contact on solar cell.

The main objective of this doctoral thesis is to the study, design and develop reliable schemes for direct writing of electronics and photovoltaics in planar geometry, by means of Laser Induced Forward Transfer (LIFT) and post deposit firing/curing techniques. And the proof of the concept will be realized on a final device of CIGS solar cell. In this research, both nanosecond and picosecond lasers have been implemented to heat a thin silver paste layer from the backside of its optically transparent glass substrate. The incident laser heat diffusion is partially absorbed by the glass slide and the rest were transferred to the silver pastes and propel them to the wafer surface. The silver paste form droplet then lifts off from paste/glass interface, forming voxel on the acceptor. Then by overlapping the laser pulses with a proper pitch distance, fine line with high aspect ratio will be directly printed. In addition, we develop a laser-based process for firing (sintering) the metallic pastes predeposited by LIFT, avoiding the time consuming standard step of a 
thermal cofiring of the silver paste. For that process, standard CW lasers can be used to design a solution industrially viable to integrate in the same machine the LIFT process and the laser firing process.

The outline of this thesis is as follows. In addition to the brief introduction in this chapter, chapter 2 reviews the background of metallization process by addressing the influence on solar cell and the several front contact metallization methods is discussed. To the end, an overview of LIFT based on the transferred material is given. Chapter 3 gives a review of Laser-induced forward transfer (LIFT) technique. Chapter 4 introduces the experimental and characterization method used to investigate the experimental results of the LIFT process in this thesis work. The experimental set-up and electrical characterization method is presented.

Chapter 5 addresses the experimental result with nanosecond laser from voxel printing to line printing. Influences of process parameter like laser fluence, film thickness are discussed in detail. Moreover, the transfer mechanism of the high viscosity silver paste is revealed by the time-resolved imaging study. Chapter 6 focuses on the picosecond laser experimental results, especially the influence of the laser energy, gap distance, and the pitch distance of line printing. In the end, a comparison of ns- and psLIFT results is discussed. In chapter 7, the sintering results with CW lasers are studied for the functionalization of the LIFT line. And the objective of the proof of metallization using LIFT on the final device, which is the CIGS solar cell, is presented in chapter 8 . Chapter 9 concludes the experimental findings and further research works and development is provided. 


\section{Background}

\subsection{Influence of metallization process on efficiency of solar cell}

Metallization is regarded as one of the key but second most expensive step in the typical solar cell fabrication. In a solar cell structure, metallization process consists of front side metallization and back side metallization. Gridlines (fingers and busbars) and back contact has to be well structured in order to achieve a high efficiency solar cell. High quality contact is required to improve all fill factor (FF) related parameters, such as the series $\left(R_{s}\right)$ and shunt resistance $\left(R_{s h}\right)$, ideality factor $(n)$ which typically has a value between 1 and 2 , and dark saturation current $\left(I_{0}\right)$. From the electrical point of view, we can analysis the influence of these parameters on the efficiency of a solar cell. Equation (2.1) is the general expression for the current-voltage $(I-V)$ characteristic of a typical solar cell with parasitic resistance according to the circuit model (see Fig. 2.1),

$$
I=I_{S C}^{\prime}-I_{o}\left(e^{q\left(V+\frac{I R_{S}}{n k T}\right)}-1\right)-\frac{\left(V+I R_{S}\right)}{R_{S h}}
$$

where $I_{S C}^{\prime}$ is the short-circuit current when there are no parasitic resistances, $I$ is current, $V$ is the voltage, $R_{S}$ is the series resistance, $R_{S h}$ is the shunt resistance, $k$ is Boltzmann constant, $T$ is the cell temperature, and $q$ is the absolute value of the electron charge [20]. 


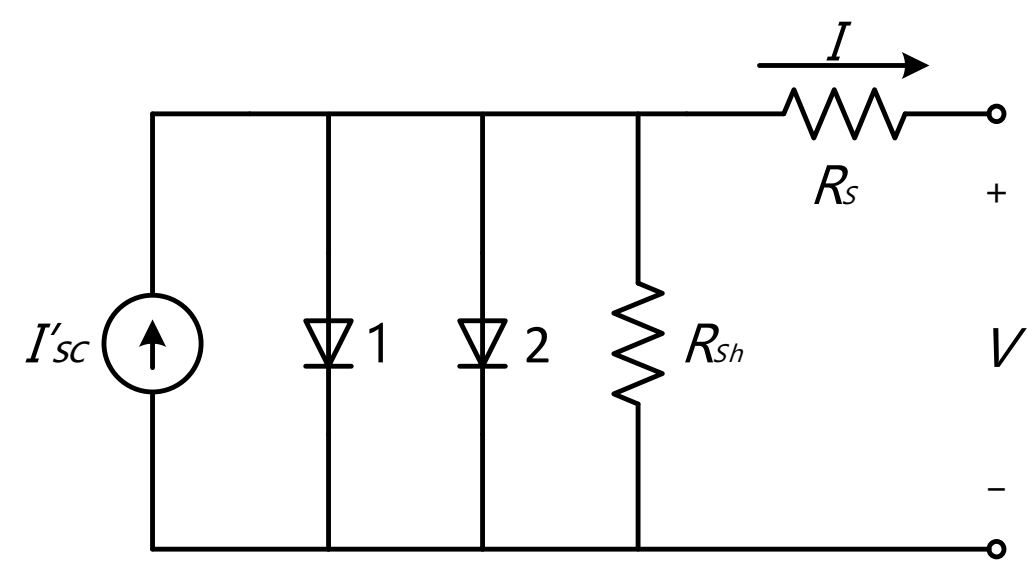

Figure 2.1: Solar cell circuit model with parasitic resistances [20].

At short-circuit, Equation (2.1) becomes

$$
I_{S C}=I_{S C}^{\prime}-I_{o}\left(e^{\frac{q I_{S C} R_{S}}{n k T}}-1\right)-\frac{I_{S C} R_{S}}{R_{S h}}
$$

As can be seen from Equation 2.2, increase series resistance will not affect the opencircuit voltage but reduce the short-circuit current. The effect of the parasitic resistances on the $I-V$ characteristic is shown in Fig. 2.2 [20]. 


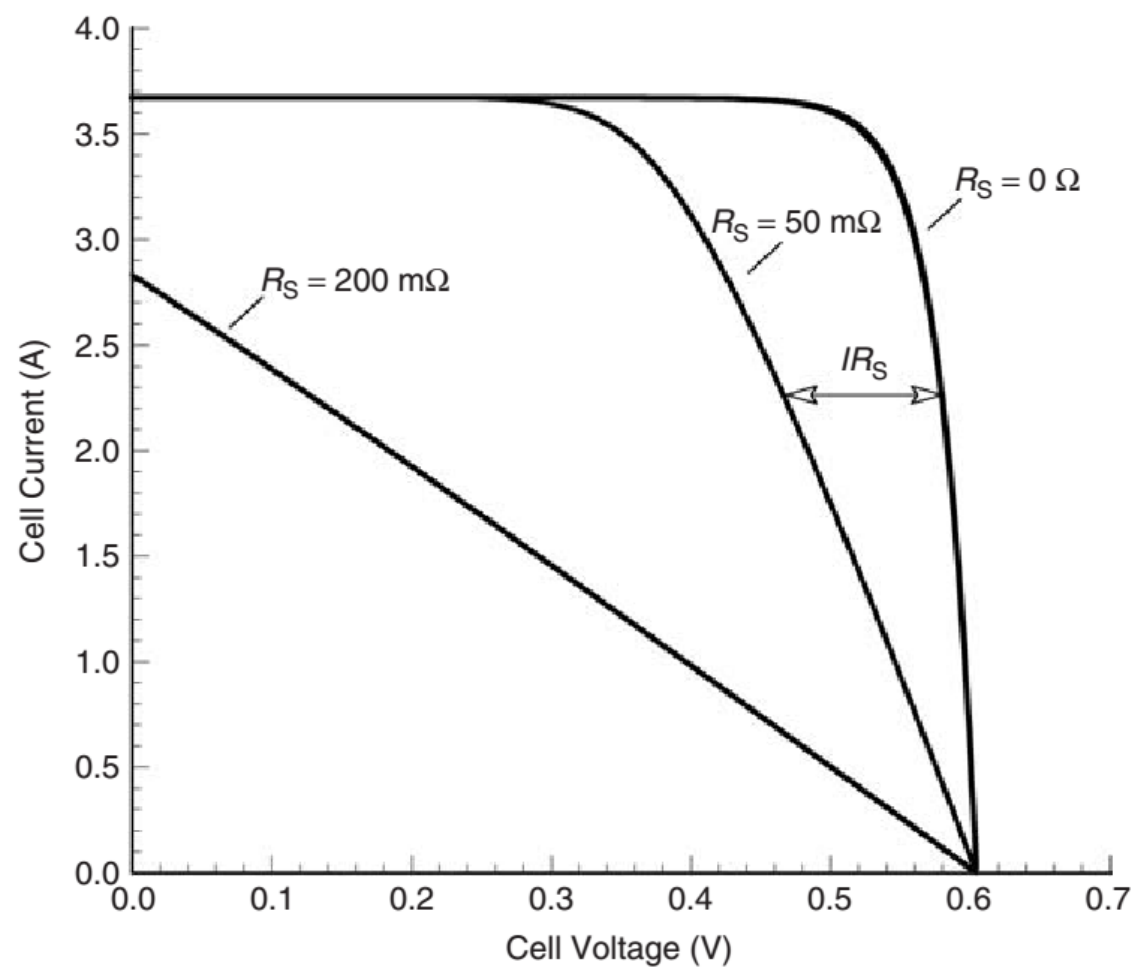

Figure 2.2: Effect of series resistance on the current-voltage characteristic of a solar cell $\left(R_{S h} \rightarrow \infty\right)[20]$.

And at open-circuit, it becomes,

$$
0=I_{S C}^{\prime}-I_{o}\left(e^{\frac{V_{o C}}{n k T}}-1\right)-\frac{V_{o c}}{R_{S h}}
$$

where $V_{o c}$ is the open-circuit voltage. By combine Equation (2.1) and (2.2), (2.3) can been obtained in the regime where series resistance is important

$$
I_{S C} R_{S}=\frac{n k T}{q} \ln \left(\frac{I_{0} e^{q V_{o c} / n k T}-I_{S C}}{I_{0}}\right)
$$


Here $r_{s}$ is defined as the normalized series resistance by $R_{s} / R_{c h}, R_{c h}$ is the "characteristic resistance" of a cell defined as the ratio $V_{o c} / I_{S C}$, and $v_{O C}$ is defined as the normalized voltage by $q V_{o c} / n k T$. Thus, dividing the left side of the Equation (2.4) by $V_{o c}$, the $r_{S}$ can be expressed by

$$
r_{S}=\frac{I_{S C} R_{S}}{V_{o c}}=\frac{1}{v_{o c}} \ln \left(\frac{I_{0} e^{q V_{o c} / n k T}-I_{S C}}{I_{0}}\right)
$$

For a cell which the shunt resistance $R_{s h}$ is very large to be negligible, $R_{S}$ appears to be more important. And the ideal fill factor $F F_{0}$ in the absence of series resistance, can be expressed with a semi-empirical expression [20] as

$$
F F_{0}=\frac{V_{o c}-\ln \left(q V_{o c} / \mathrm{kT}+0.72\right)}{V_{o c}+\mathrm{kT} / \mathrm{q}}
$$

Considering the effect of $r_{S}$ on $F F$, the approximate and empirical expression for the effect of this resistance $F F_{S}$ can be allowed by writing

$$
F F_{S}=F F_{0}\left(1-r_{s}\right)
$$

As discussed before and can been seen from Equation (2.4) and (2.5), increase $R_{S}$ leads to the decrease of $I_{S C}$ and cause the increase of $r_{S}$. And $V_{O C}$ will not be affected so that $F F_{0}$ will not change, which results in reducing the fill factor value. 


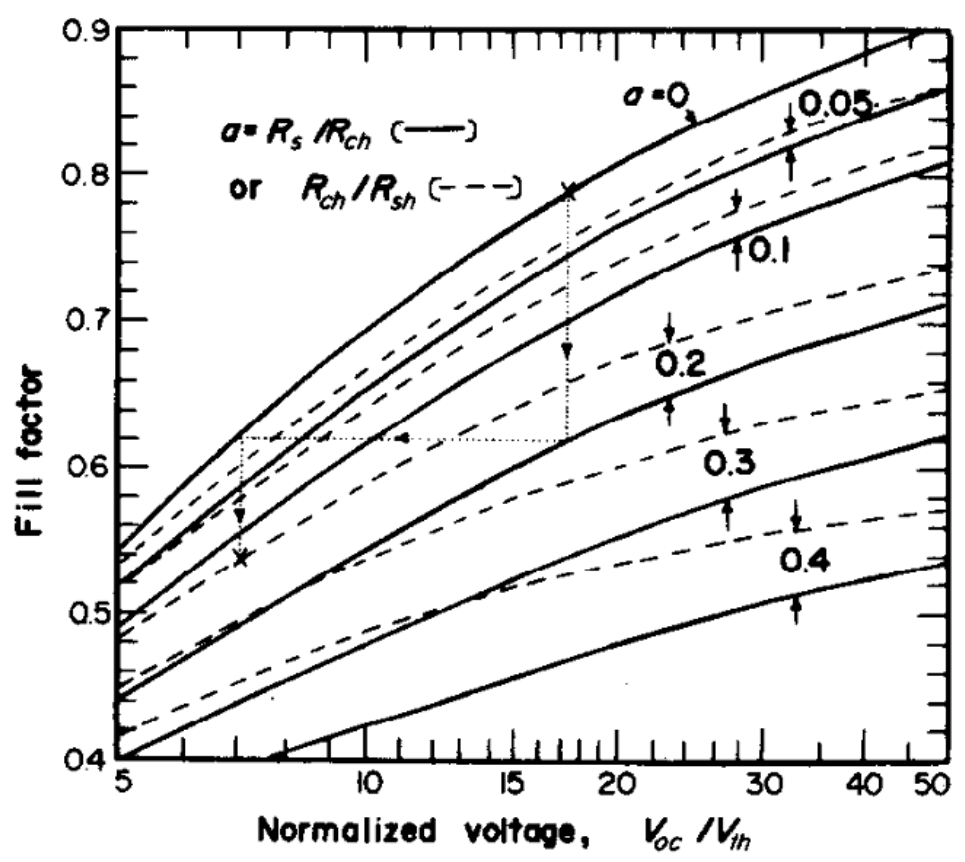

Figure 2.3: The general solar cell fill factor diagram. $\mathrm{V}_{\text {th }}$ is $\mathrm{nkT} / \mathrm{q}$ [21].

And from this figure of merit, a further understanding of the influence of series resistance to solar cell efficiency can be obtained from the efficiency equation as

$$
\eta=\frac{P_{\max }}{P_{\text {in }}}=\frac{F F \cdot V_{o c} \cdot I_{S C}}{P_{\text {in }}}
$$

The incident power $P_{\text {in }}$ is determined by the light spectrum incident upon the solar cell. With increasing the series resistance, the maximum power of the solar cell $\left(P_{\max }\right)$ will be decreased by the products of $I_{S C}, V_{o c}$ and $F F$.

Hence, it can be concluded that series resistance has a great electrical affect upon short-circuit current $\left(I_{S c}\right)$, fill factor $(F F)$ and cell efficiency $(\eta)$. And the cell efficiency is by far the most important figure of merit. 


\subsection{Influence of metal contact geometry on power loss and series resistance}

Power loss is caused by series resistance in the cell, related with the current travels though the diffused sheet, across the metal-semiconductor interface, along the grid fingers and busbar, and through the base of the cell.

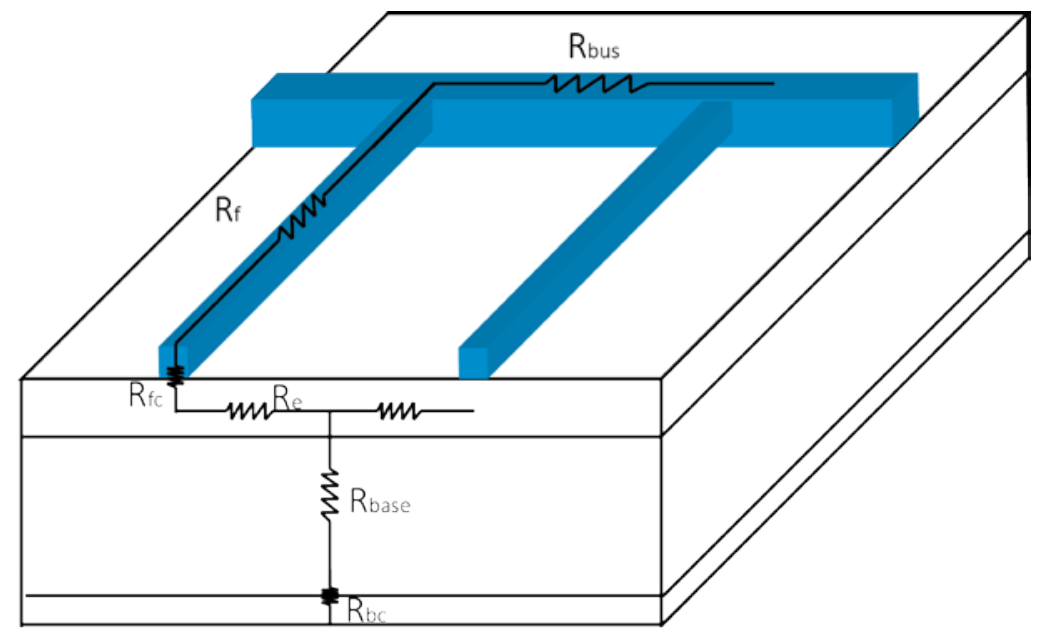

Figure 2.4: Schematic diagram of series resistance distribution on solar cell.

The total series resistance is then the sum of all the mentioned terms, expressed as

$$
R_{s}=R_{b u s}+R_{f}+R_{f c}+R_{e}+R_{b a s e}+R_{b c}
$$

and the series resistance related with the front contact metallization is (2.10)

$$
R_{\text {sfront }}=R_{\text {bus }}+R_{f}+R_{f c}
$$

In order to study the front contact influence, a simple contact grid structure [21] can be made to quantify the various power loss components and evaluate the contact parameters design of cell by the derived expressions. 


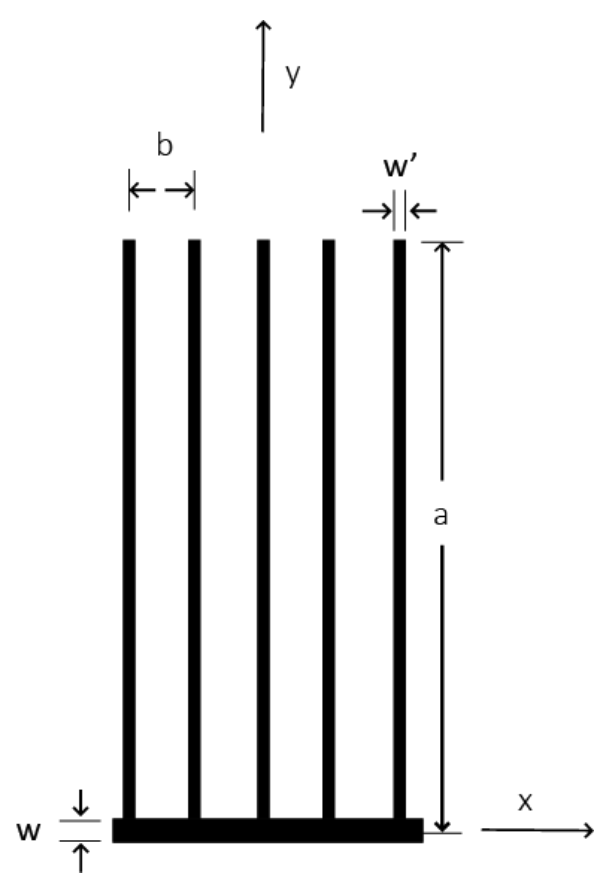

Figure 2.5: Schematic diagram of a simple pattern of front contact. Adapted from [21].

Fig. 2.5 shows the simple contact grid pattern, which is applied to a solar cell with $L$ units long and $n S$ units wide, where $n$ is the number of grid fingers and $S$ is the finger spacing. Current flows from the contact pad at the center of the bus bar. In this model, the power loss associated with the current travelling along the diffused layer can be written as follows [21]:

$$
P_{\text {sheet }}=2 n \int_{0}^{b} I^{2}(x) d R
$$

$d R$ is the resistance of $d x$ wide and $\mathrm{L}$ units high at position $x, I(x)$ is the current as a function of $x$ in the same strip, these can be expressed by (2.12)

$$
d \mathrm{R}=\left(\rho_{E} d x / \mathrm{L}\right)
$$




$$
I(y)=\int_{x}^{b} J_{m p p} L d x^{\prime}=J_{m p p}(\mathrm{~b}-x) L
$$

where $\rho_{E}$ is the semiconductor sheet resistivity and $J_{L}$ is light-generated current density. Substituting (2.12) and (2.13) into (2.11) we can obtain

$$
P_{\text {sheet }}=\frac{n \mathrm{~L} S^{3} J_{m p p}^{2} \rho_{E}}{12}
$$

In the grid fingers, power loss can be expressed as

$$
P_{\text {finger }}=n \int_{0}^{a} I^{2}(y) d R
$$

$I(y)$ is the accumulated current in the fingers at location $y$,

$$
I(y)=\int_{a}^{y} J_{L} b d y^{\prime}=J_{m p p}(y-a) b
$$

$d R_{f}$ is the element of grid finger which is represented by

$$
d R_{f}=\left(\frac{\rho_{f}}{A_{f}}\right) d y
$$

where $\rho_{f}$ is the resistivity of the grid is finger material and $A_{f}$ is the cross-sectional area of the finger. Substituting (2.16)and (2.17) into (2.15) we can obtain

$$
P_{\text {finger }}=\frac{n L^{3} S^{2} J_{m p p}^{2} \rho_{f}}{3 h w}
$$


where $h$ and $w$ are the height (thickness) and width of the grid finger.

The power loss associated with busbar can be treated similarly to the grid fingers. Although the current enters the busbar in discrete quantities from individual grid lines, it can be treated as continuous current from grid, collected in the busbar and flows along busbar and enters in the contact pad at center of busbar.

$$
\begin{gathered}
P_{\text {busbar }}=\int_{0}^{n S} I_{b}^{2}(x) d R_{b} \\
I_{b}^{2}(x)=\int_{n S / 2}^{x} J_{L} a d x=J_{m p p} a(x-n b / 2) \\
d R_{b}=\left(\frac{\rho_{f}}{A_{b}}\right) d x
\end{gathered}
$$

substituting (2.20) and (2.21) into (2.19) gives

$$
P_{\text {busbar }}=\frac{n^{3} L^{2} S^{3} J_{m p p}^{2} \rho_{f}}{12 h^{\prime} w^{\prime}}
$$

and the power loss resulting from the shadowing effect by the fingers and busbars is

$$
P_{\text {shadow }}=J_{m p p} V_{m p p} n\left(L w+S w^{\prime}\right)
$$

The power loss associated with contact resistance is

$$
P_{\text {contact }}=2 n I^{2} R_{c}
$$




$$
\begin{array}{r}
I=\frac{J_{L} L S}{2} \\
R_{C}=\frac{\left(\rho_{c} \rho_{S}\right)^{\frac{1}{2}}}{L}
\end{array}
$$

substituting (2.25) and (2.26) into (2.24) yields

$$
P_{\text {contact }}=\frac{J_{m p p}^{2} n L S^{2}\left(\rho_{c} \rho_{s}\right)^{\frac{1}{2}}}{2}
$$

where $\rho_{c}$ is the contact resistivity $R_{c}$ is the contact resistance and $R_{S}$ is the sheet resistance.

Hence the total power loss caused by the front contact metallization can be expressed by

$$
\begin{gathered}
P_{\text {front }}=P_{\text {finger }}+P_{\text {busbar }}+P_{\text {shadow }}+P_{\text {contact }}+P_{\text {sheet }} \\
=J_{m p p} V_{m p p} n L w+\frac{n L^{3} S^{2} J_{m p p}^{2} \rho_{f}}{3 h w}+J_{m p p} V_{m p p} n S w^{\prime}+\frac{n^{3} L^{2} S^{3} J_{m p p}^{2} \rho_{f}}{12 h^{\prime} w^{\prime}} \\
+\frac{J_{m p p}^{2} n L S^{2}\left(\rho_{c} \rho_{s}\right)^{\frac{1}{2}}}{2}+(1 / 12) n L S^{3} J_{m p p}^{2} \rho_{E}
\end{gathered}
$$

From Equation (2.28), it can be seen that the power loss of the front line is related to the numbers of the grid finger $n$, the length of the grid finger $L$, the finger spacing $S$, the contact line width $w$ and $w^{\prime}$, and the contact line height $h$ and $h^{\prime}$. If the gridline width is a constant, increasing the contact height will decrease the power loss. On the other hand, if the height is fixed, increase the contact line width could give rise to the power loss caused by shadowing. The competition between shading effect and line resistivity can yield a minimum value of the power loss expression of front contact. Considering solely 
the defining of front contact geometry, we can regard the line width as variable and other parameters as constants, the minimum power loss expression yields.

$$
\begin{gathered}
P_{\text {front }(\text { min })}=P_{\text {finger }(\text { min })}+P_{\text {busbar }(\text { min })}+P_{\text {contact }} \\
=\frac{2 \sqrt{3}}{3} \mathrm{n} L^{2} S J_{L} \sqrt{\frac{J_{m p p} V_{m p p_{L}} \rho_{f}}{h}+\frac{\sqrt{3}}{3} n^{2} L S^{2} J \sqrt{\frac{J_{m p p} V_{m p p} \rho_{f}}{h^{\prime}}}} \\
+\frac{J_{m p p}^{2} n L S^{2}\left(\rho_{c} \rho_{s}\right)^{\frac{1}{2}}}{2} \\
=\frac{\sqrt{3 \eta P_{L} \rho_{f}}}{3} n L S J_{m p p}(2 L+n S)\left(\sqrt{\frac{1}{h}}+\sqrt{\frac{1}{h^{\prime}}}\right)+\frac{J_{m p p}^{2} n L S^{2}\left(\rho_{c} \rho_{s}\right)^{\frac{1}{2}}}{2} \\
w_{\min }=L S J_{m p p} \sqrt{\frac{\rho_{f}}{3 \eta P_{L}}} \sqrt{\frac{1}{h}} \\
w_{\min }^{\prime}=\frac{n}{2} L S J_{m p p} \sqrt{\frac{\rho_{f}}{3 \eta P_{m p p}}} \sqrt{\frac{1}{h^{\prime}}}
\end{gathered}
$$

By defining aspect ratio of the grid line $\Phi=h / w$, the height and cross-sectional area can be expressed by

$$
\begin{gathered}
h=w \Phi \\
A=w h=w^{2} \Phi
\end{gathered}
$$

and the Substituting (2.32) into (2.29), (2.30) and (2.31) yields

$$
w_{\text {min }}=\sqrt[3]{\frac{L^{2} S^{2} J_{m p p}^{2} \rho_{f}}{3 \eta P_{L} \Phi}}
$$




$$
\begin{gathered}
w_{\text {min }}^{\prime}=\sqrt[3]{\frac{n^{2} L^{2} S^{2} J_{m p p}^{2} \rho_{f}}{12 \eta P_{L} \Phi}} \\
P_{\text {front (min })=} \sqrt[3]{\frac{1}{\Phi} \frac{\sqrt{3 \eta P_{L} \rho_{f}}}{3}} n L S J_{L}(2 L+n S)\left(\sqrt[6]{\frac{3 \eta P_{L}}{L^{2} S^{2} J_{L}^{2} \rho_{f}}}+\sqrt[6]{\frac{12 \eta P_{L}}{n^{2} L^{2} S^{2} J_{L}^{2} \rho_{f}}}\right) \\
+\frac{J_{L}^{2} n L S^{2}\left(\rho_{c} \rho_{s}\right)^{\frac{1}{2}}}{2}
\end{gathered}
$$

Hence increasing the aspect ratio of gridline can decrease the minimum value of both line width and power loss, and the corresponding $w_{\min }$ is also need to be decreased. If $w>$ $w_{\text {min }}$, minimize the width and increase the aspect ratio is the efficient way to reduce the power loss caused by front contacts.

The series resistance, normalized to unit cell area ( $\mathrm{LLS})$, can be derived by

$$
r_{S}=\frac{P_{\text {total }}}{J_{L}^{2} n L S}
$$

Hence the $r_{s}$ (finger) and $r_{s}$ (busbar) can be expressed by

$$
\begin{gathered}
r_{S}(\text { finger })=\frac{n L^{2} S J_{L}^{2} \rho_{f}}{3 h w}=\frac{n L^{2} S J_{L}^{2} \rho_{f}}{3 w^{2} \Phi} \\
r_{S}(\text { busbar })=\frac{n^{2} L S^{2} J_{L}^{2} \rho_{f}}{12 h^{\prime} w^{\prime}}=\frac{n^{2} L S^{2} J_{L}^{2} \rho_{f}}{12 w^{\prime 2} \Phi}
\end{gathered}
$$

Increasing the cross-sectional area can reduce the series resistance of front contact. As the gridline widths are confined by the shadowing to the $w_{\min }$ and $w_{\min }^{\prime}$, the effective 
way to reduce the minimum series resistance of front contact is also to increase the aspect ratio.

The total power loss terms can be normalized to obtain the fractional power loss of each loss component. The total power generated by the model is expressed as

$$
P_{\text {total }}=n J_{m p p} V_{m p p} S L
$$

The power loss due to the finger can be normalized to (2.42) resulting in a fractional power loss due to the finger.

$$
\begin{gathered}
\Delta P_{F, \text { total }}=\frac{P_{\text {finger }}+P_{\text {busbar }}+P_{\text {shadow }}+P_{\text {contact }}+P_{\text {sheet }}}{P_{\text {total }}} \\
=\left(J_{m p p} V_{m p p} n L w+\frac{n L^{3} S^{2} J_{m p p}^{2} \rho_{f}}{3 h w}+J_{m p p} V_{m p p} n S w^{\prime}+\frac{n^{3} L^{2} S^{3} J_{m p p}^{2} \rho_{f}}{12 h^{\prime} w^{\prime}}\right. \\
\left.+\frac{J_{m p p}^{2} n L S^{2}\left(\rho_{c} \rho_{s}\right)^{\frac{1}{2}}}{2}+\left(\frac{1}{12}\right) n L S^{3} J_{m p p}^{2} \rho_{s}\right) / J_{m p p} V_{m p p} n S L \\
=\frac{w}{S}+\frac{L^{2} S J_{m p p} \rho_{f}}{3 V_{m p p} h w}+\frac{w^{\prime}}{L}+\frac{n^{2} \rho_{f} J_{m p p} L S^{2}}{12 V_{m p p} h^{\prime} w^{\prime}}+\frac{J_{m p p} S\left(\rho_{c} \rho_{s}\right)^{\frac{1}{2}}}{2 V_{m p p}}+\frac{J_{m p p} S^{2} \rho_{s}}{12 V_{m p p}}
\end{gathered}
$$

To apply this model, the parameters related with the materials and electrical characterizations should be specified for the assumption. The values applied in the study can be seen in Table 2.1.

\begin{tabular}{|c|c|c|c|c|c|c|c|c|c|}
\hline $\mathrm{S}$ & $\mathrm{L}$ & $\mathrm{n}$ & $w^{\prime}$ & $\Phi$ & $\rho_{f}$ & $\rho_{c}$ & $\rho_{s}$ & $J_{m p p}$ & $V_{m p p}$ \\
\hline $0.2 \mathrm{~cm}$ & $3 \mathrm{~cm}$ & 2 & $0.2 \mathrm{~cm}$ & 0.1 & $6 \mu \Omega \cdot \mathrm{cm}$ & $\begin{array}{c}20 \\
\mathrm{~m} \Omega \cdot \mathrm{cm}^{2}\end{array}$ & $60 \Omega / \square$ & $25 \mathrm{~mA} / \mathrm{cm}^{2}$ & $0.5 \mathrm{~V}$ \\
\hline
\end{tabular}

Table 2.1: Material and solar cell operating properties used in the grid geometric analysis [20]. 


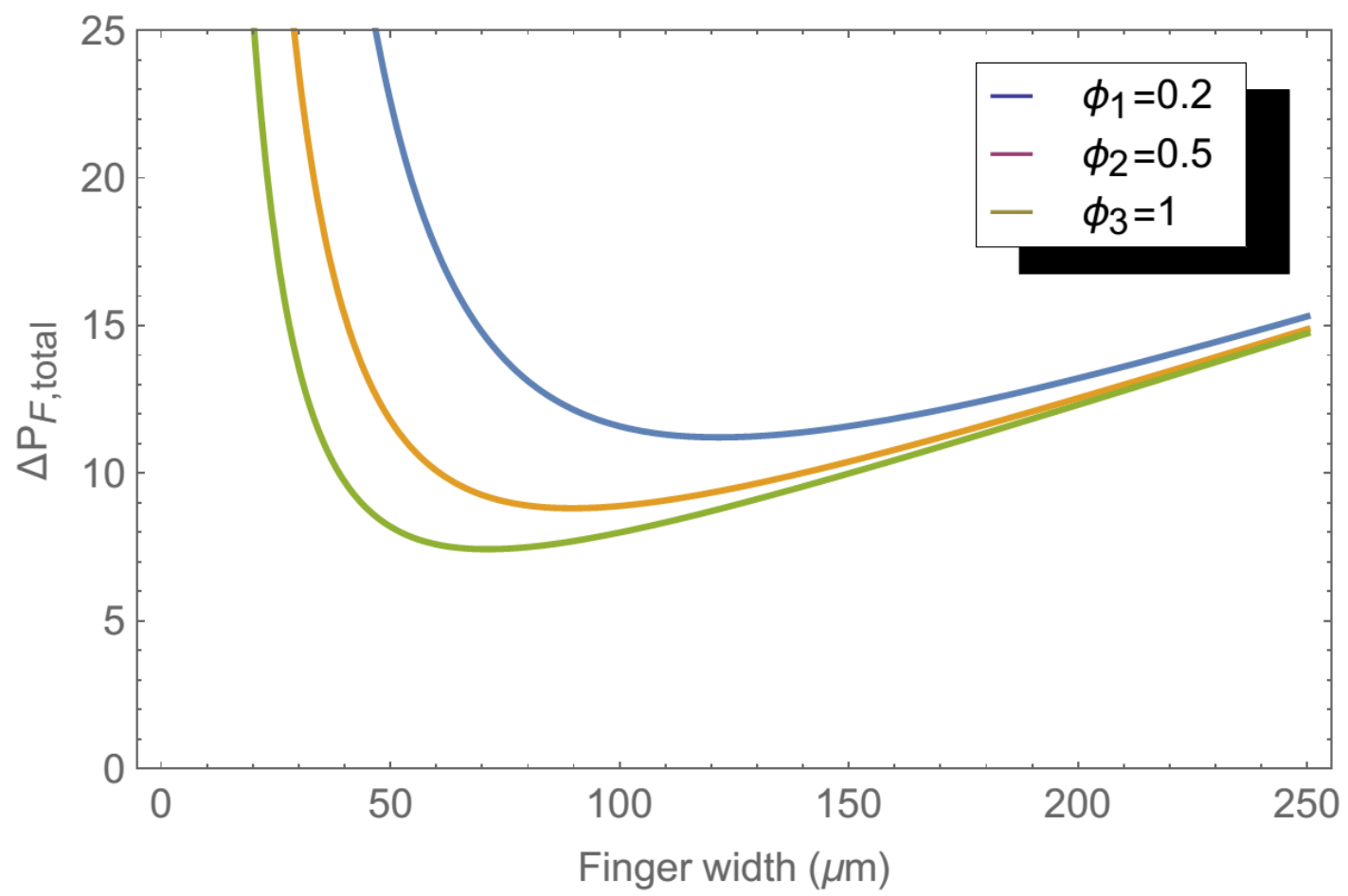

Figure 2.6: Power loss fraction as a function of finger width in context of three different aspect ratios.

\subsection{Front contact metallization methods}

The front contact metallization, which has the proved competence to enhance the efficiency and reduce the cost, is widely under investigation. The metallization process has both optical and electrical impact to the performance of solar cell. The finger line width contributes to the shading effect, which present a smaller short circuit current. And electrically both front and rear contact influence the series resistance through contact and finger line resistance, which corresponds to the fill factor, as was presented previously. 
In industrial cell production nowadays, screen-printing provides a well-established, robust and rapid method to deposit metal contacts. It has been developed for decades and efforts have been made to understand the contact mechanisms to improve the cell efficiency in this mature technique since 1970s. Although various advanced screen printing techniques have been evolved to overcome the drawback of the conventional one [22-25], like the high grid width (more than $100 \mu \mathrm{m}$ ), poor conductivity and aspect ratio which confine the fill factor to not more than $77 \%$. Despite the limitations, because of its simplicity, high productivity and cost effectiveness screen printing is the most commonly implemented metallization technique in industry till date.

On the other hand, with laboratory processes such as photolithography, the defined contacts present the best performance like high aspect ratio, high resolution and high line conductivity which finally contributes to obtain a high efficiency solar cell [26]. But the complex steps and expensive cost result in a non-manufacturable technique for mass production.

Hence, some non-contact printing processes with cost-effective feature tend to be preferable for fine line metallization. Several different printing techniques have been developed to abridge the enormous gap between high-efficiency laboratory technique and the industrial implementation. Metallization process can be further classified into two steps [27]: pre-metallization and subsequent metallization. The pre-metallization refers to the selective patterning of dielectric layer and the development of the metal seed layer, while the subsequent metallization is commonly applied in a two-step metallization process aiming at functionalizing the metal contact by drying, curing, firing or sintering, and improving the line conductivity by thickening the line, using plating process for providing sufficient lateral current transport [27]. According to the deposition method, there are two routes of front contact pre-metallization: contact and non-contact methods. Contact method refers to the mechanical or chemical contact during the metal seed growth step which bring the residues or byproducts to the substrate. 
The classification of pre-metallization and its sub-classification are depicted in Fig. 2.7. And in principle, each process and the comparison of different method are discussed in the respective section.

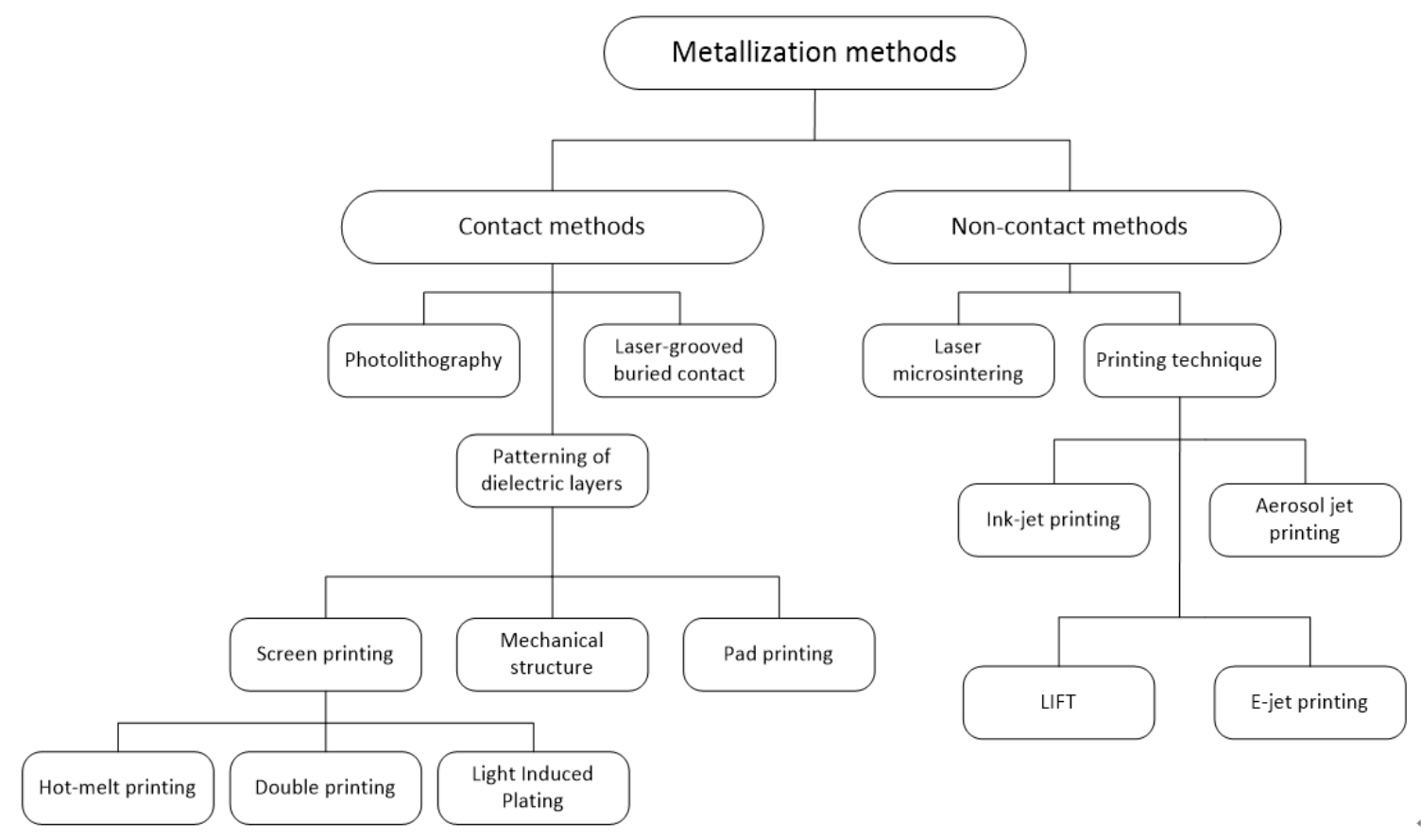

Figure 2.7: The classification of metallization method. Adapted from [27]

\subsubsection{Photolithographically defined contacts}

Photolithographical definition technology presents the best contact performance in the PERL structure of high-efficiency solar cell. By using this method Jianhua et al. [28] obtained an efficiencies of $24.7 \%$ on single crystalline silicon and $20.3 \%$ on cast multicrystalline silicon. Like the typical procedure of photolithography, a positive photoresist layer is firstly deposited by spin-coating on the AR layer of the Si solar cell. After drying off the solvent, a mask of the designed front contact pattern is applied on top of the photoresist and then exposed to UV light. The illuminated area becomes soluble and is washed out in the photographic developer. After the exposure, typically an additional post develop bake (or "hard bake") of the photoresist pattern is applied for 
stabilizing the printed feature for the following wet chemical etch process of AR layer. The formed pattern groove is then deposited by high vacuum evaporation process of pure metal or a stack layer of metal. The stack metal system typically consists of titanium, palladium and silver. Titanium stays at bottom for its low contact resistance of less than $10^{-5} \Omega \cdot \mathrm{cm}^{2}$ to the $\mathrm{n}$-doped emitter and providing a good adhesion contact. Palladium serves as a diffusion barrier and intermediate layer for promoting adhesion between titanium and silver. And the silver is used for transporting current for its low bulk resistivity. Finally, the excess of metals and photoresist are removed by a solvent, like acetone, which chemically alter the adhesion of the photoresist to the substrate. The evaporated thin metal layer is then thickened by a plating step and sintered.

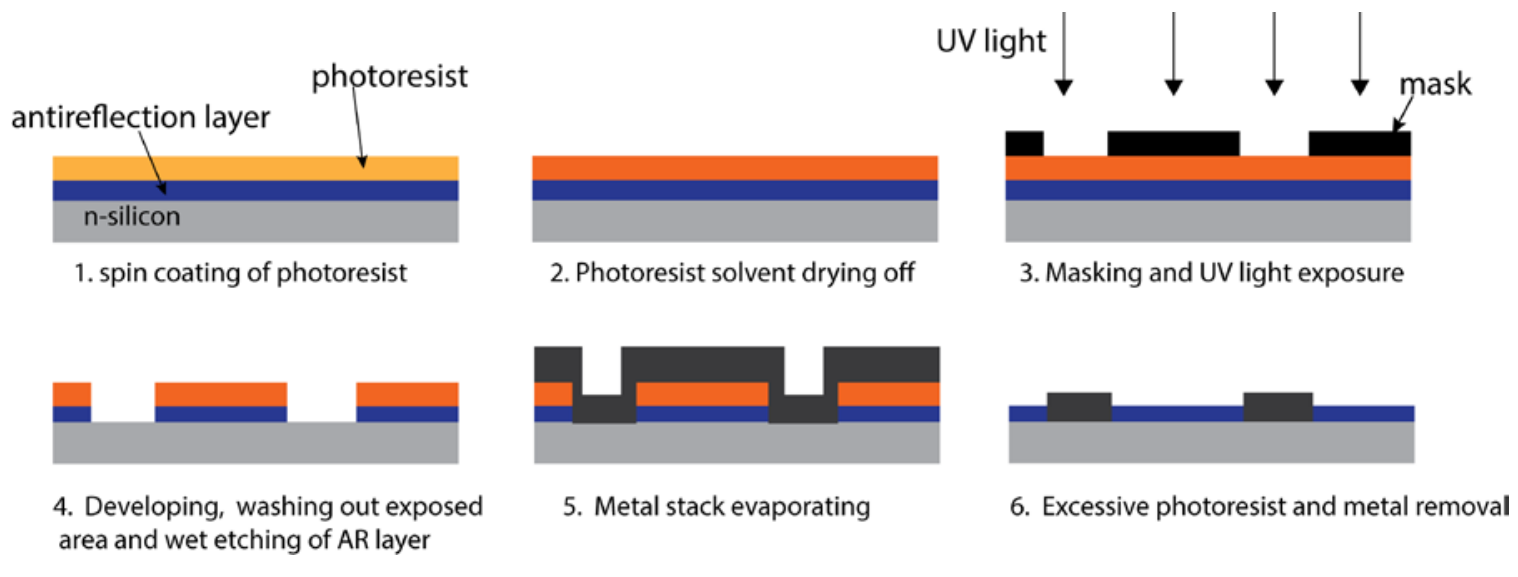

Figure 2.8: Scheme of photolithography and metal evaporation process for the front side metallization.

\subsubsection{Laser grooved buried contact}

The laser grooved buried contact (LGBC, see Fig. 2.9) technology was developed in the 1985s at University of New South Wales to produce fine lines with high aspect ratios and high line conductivity for the high efficiency solar cell, saving complex fabrication steps (Fig. 2.8) and expensive sacrificing materials cost like photoresist and masks. The grooves on the silicon substrate opened by laser scribing serve as mask for groove diffusion and electroless nickel and copper plating. The groove can be scribed with a width ranging from 
20 to $50 \mu \mathrm{m}$ and a depth of 10 to $60 \mu \mathrm{m}$. This allows reducing the gridline spacing to 1.25 $\mathrm{mm}$ hence minimizing the shading loss.

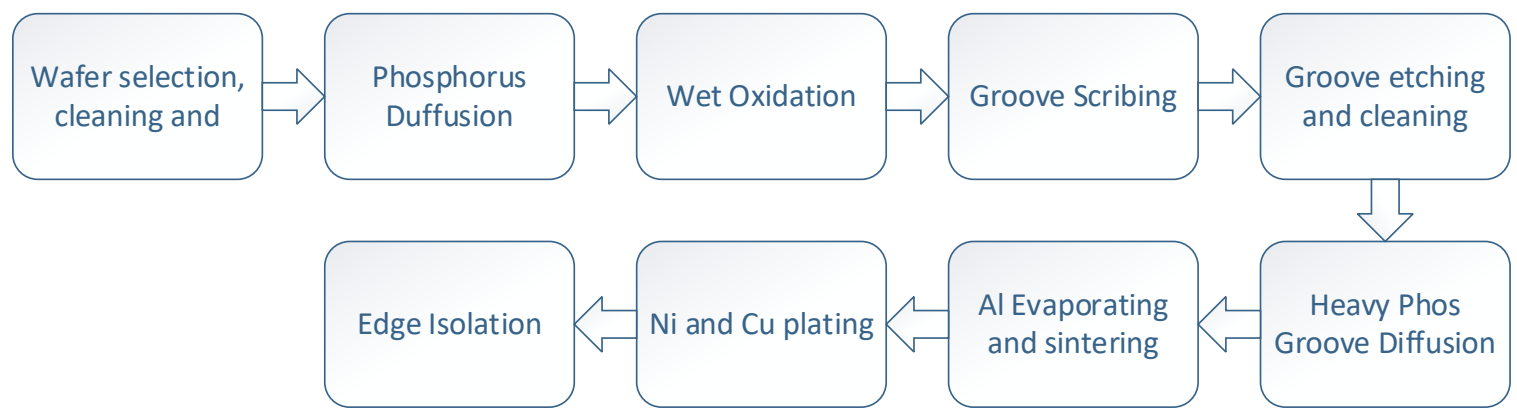

Figure 2.9: LBGC solar cell processing sequences.

The LGBC solar cell has the flexibility in choosing substrate surface texturing. This kind of cell structure also achieves many excellent features like good ohmic contacts, low shading losses (<5\%) and high cell efficiency ( $20.6 \%$ with small area cell and $19.7 \%$ with commercial area cell) [29]. Whereas, the low-quality material like multicrystalline Si and the plating process will be hard for this technique.

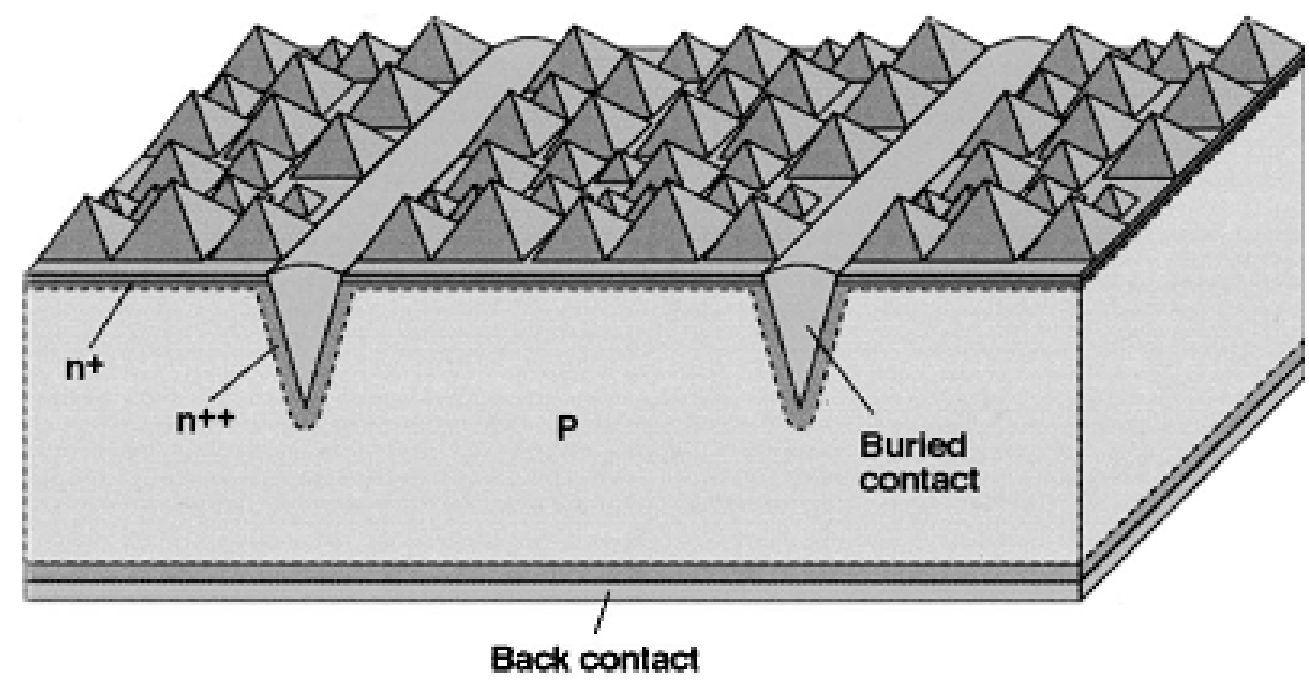

Figure 2.10: Schematic of laser grooved buried contact solar cell [30]. 


\subsubsection{Screen Printing technology}

Screen printing is the most well-established and robust technology for the front grid as well as back contact metallization process in solar cell industrial production. It is a onestep printing process, including the wafer alignment and transfer, for depositing the metal paste with the designed front grid structure in a very fast speed (less than 3 seconds) in a single industrial screen-printing line.

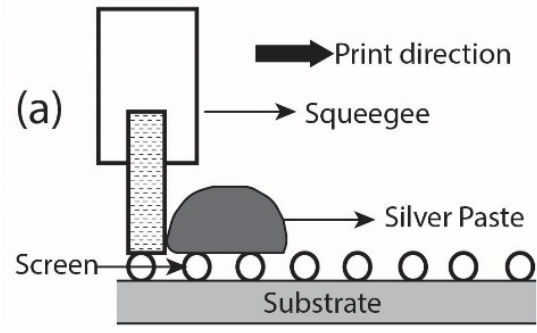

(b)

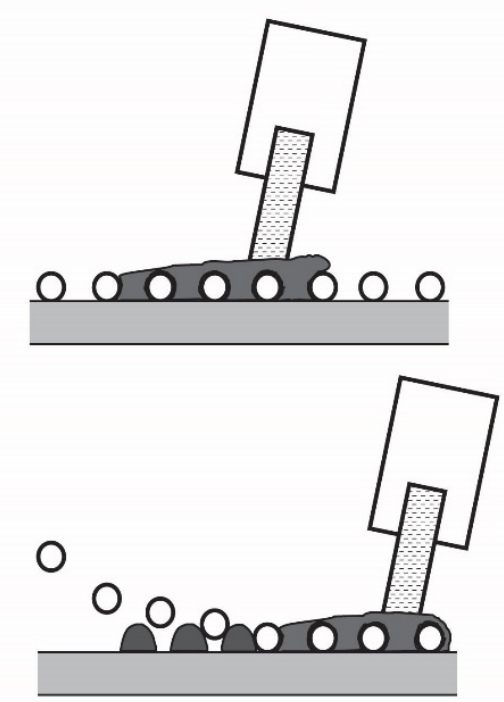

Figure 2.11: Schematic of Screen Printing process.

Fig 2.11 illustrates the schematic of the screen-printing process. As a first step, the silver pastes are flooded in front of the squeegee (floodbar) over the screen (see Fig. 2.11a).

By applying a vertical force to the printing squeegee, the screen is pressed in close contact with the substrate and the paste is squeezed through the stencil onto the surface of the 
wafer (see Fig. 2.11b). With the adhesion force, the paste sticks to the substrate surface. When releasing the screen in the final phase, the paste is released from the screen and forms the prescribed pattern (see Fig. 2.11c).

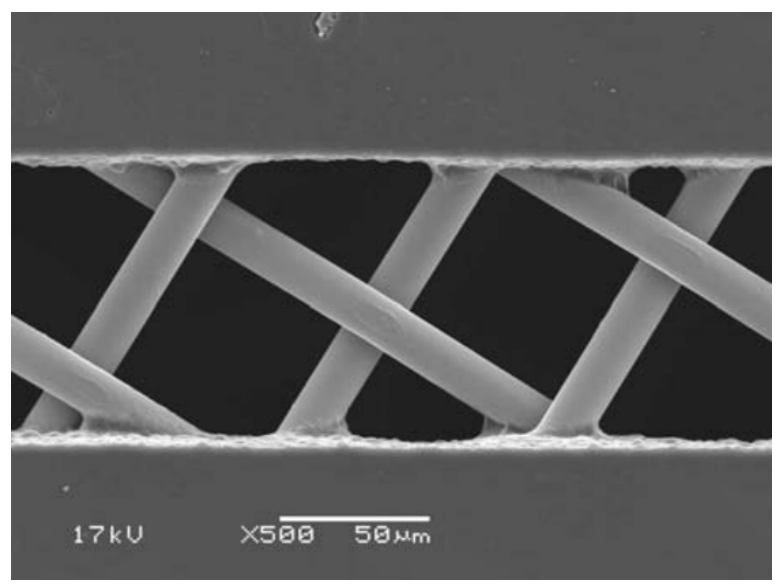

Figure 2.12: SEM images of screen printing 360 mesh screen, $15 \mu \mathrm{m}$ wire diameter, 100 $\mu \mathrm{m}$ finger line opening, screen opening fraction $\mathrm{a}_{0}$ is $60 \%$ [24].

Both screen and paste are the parameters that affect the printing quality. The screen consists of a frame made of aluminum, a mesh of steel wires, which are clamped to the frame and an emulsion layer. The emulsion layer is prescribed with the desired printing pattern. The screen opening fraction $a_{0}$, is the ratio of the screen opening area to the total area defined by

$$
a_{0}=\frac{d^{2}}{(c+d)^{2}}
$$

where $c$ is the wire thickness or diameter and $d$ is the wire separation distance. A high screen opening fraction is needed when printing fine lines. The minimum line width can be expressed by an empirical equation defined by Scheer [31] 


$$
w_{\text {fmin }}=\frac{c(2 c+d) \sqrt{2}}{d}=\sqrt{2}\left(\frac{2 c^{2}}{d}+c\right)
$$

For printing a very fine finger line of about $30 \mu \mathrm{m}$, it needs the $c$ of $14 \mu \mathrm{m}$ and $\mathrm{d}$ of 54 $\mu \mathrm{m}$. Hence, the screen utilized wire diameter is the key factor for the printing resolution. The current meshes wires diameter can be reduced below $20 \mu \mathrm{m}$ (see Fig. 2.12). The wire diameter limits the minimum mesh opening and thus the printing resolution. An alternative method for screen printing is to use stencil, which has a non-wear advantage and can printing lines with higher definitions down to $30 \mu \mathrm{m}$ [31].

Based on the screen printing technology, there are several derivation methods for improving the line quality from the point of view of aspect ratio or conductivity. Hotmelted printing is one of these methods involving a preheated print chuck for enhancing the substrate temperature during the screen printing process of the solar cell. This enables a reduced line width $(52 \mu \mathrm{m})$ and an additional gain of $1.1 \%$ relative in cell efficiency. Brirger et al. used a designed hot-melt screen printer comprising heated screen, squeegees and print tables to deposit metal paste with point of $50-80^{\circ} \mathrm{C}$. The Hot-melted screen printing shows a grid finger width of $60 \mu \mathrm{m}$ with aspect ratios ranging from 0.2 to $0.26[25]$.

By applying a second layer of paste using screen printing on the first deposited "seed" layer with high alignment accuracy, the Double Printing (DP) method can produce line with aspect ratios can be increased from 0.24 to 0.36 , resulting in a $0.22 \%$ absolute increase of cell efficiency. DP method produces fingers with very fine width (36 $\mu \mathrm{m})$ without interruption even at very narrow finger openings and high processing yield. This enables the adoption of DP method in production (see Fig. 2.13). 

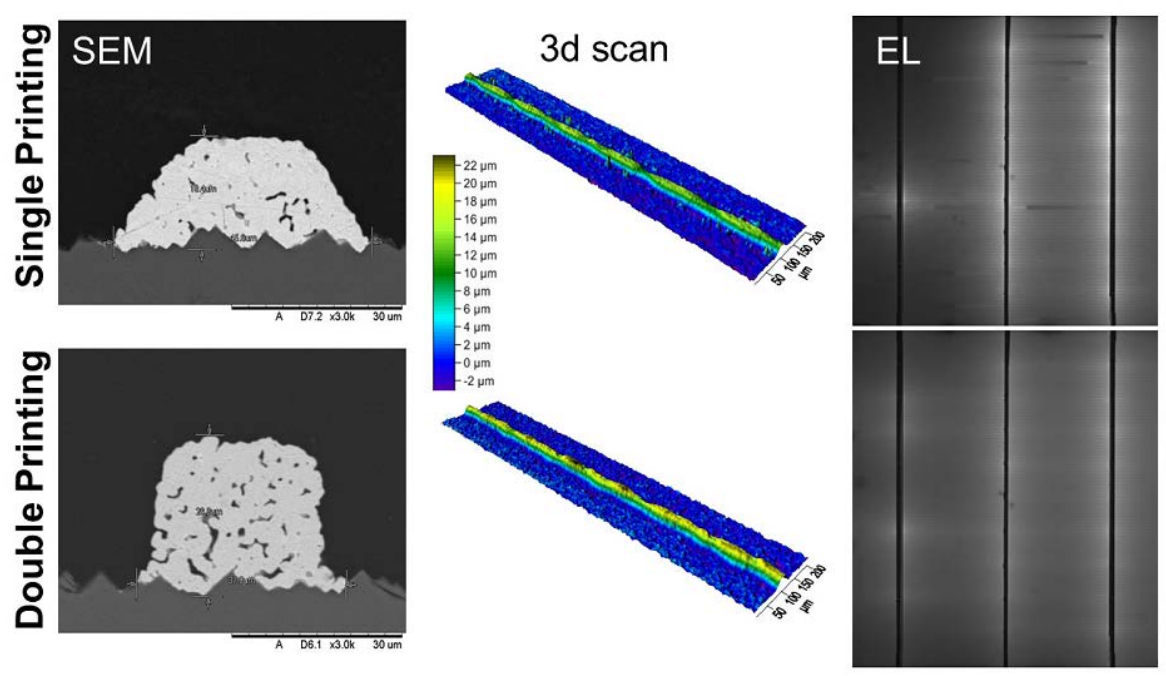

Figure 2.13: Comparisons between conventional SP (up) and DP (down) method: SEM cross section, 3D laser scan and electroluminescence images [23].

Another technique for improving the finger conductivity called light-induced plating (LIP) also works as an additional step adding a second layer on the first "seed" layer deposited by screen printing. It is a fast method for galvanizing metal contact on $\mathrm{n}$-doped material homogeneously and an inexpensive way for the reduction of using the screenprinting paste, which has been developed and optimized for years at Fraunhofer ISE [1] .

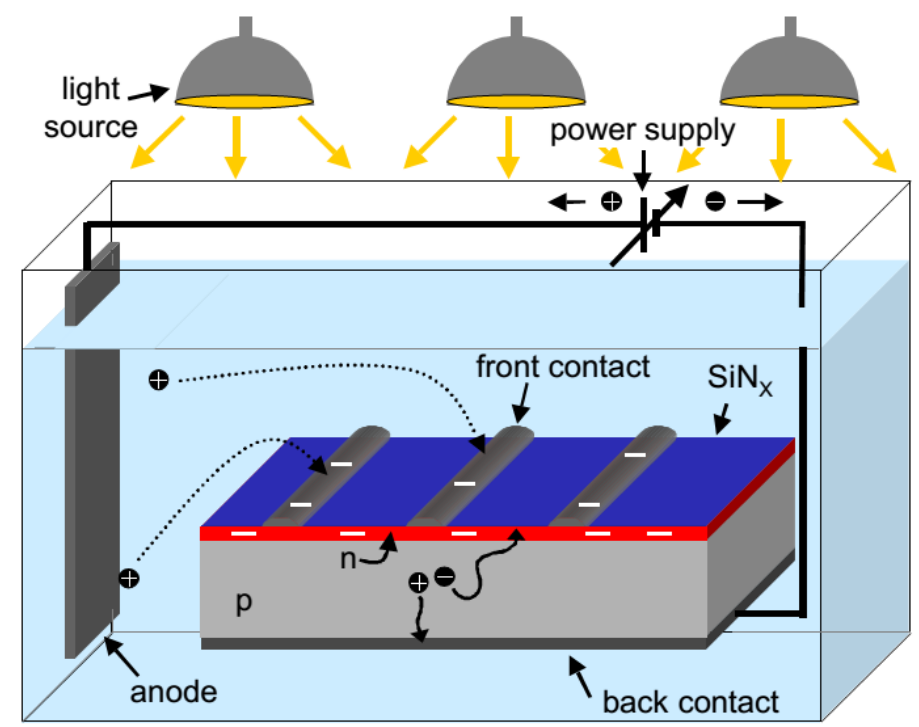

Figure 2.14: Schematic of the light-induced plating process [1]. 
In principle, the solar cell is immersed in an electroplating bath. When applying an external irradiation of light, the emitter and front silver grid itself develop a negative voltage and serve as cathode which attracts positively charged ions from the bath and thus thicken the screen-printed "seed" layer (see Fig. 2.15). By applying an external voltage, the backside of solar cell, which serves as the metal anode, will be neutralized and thus is protected from being dissolved.

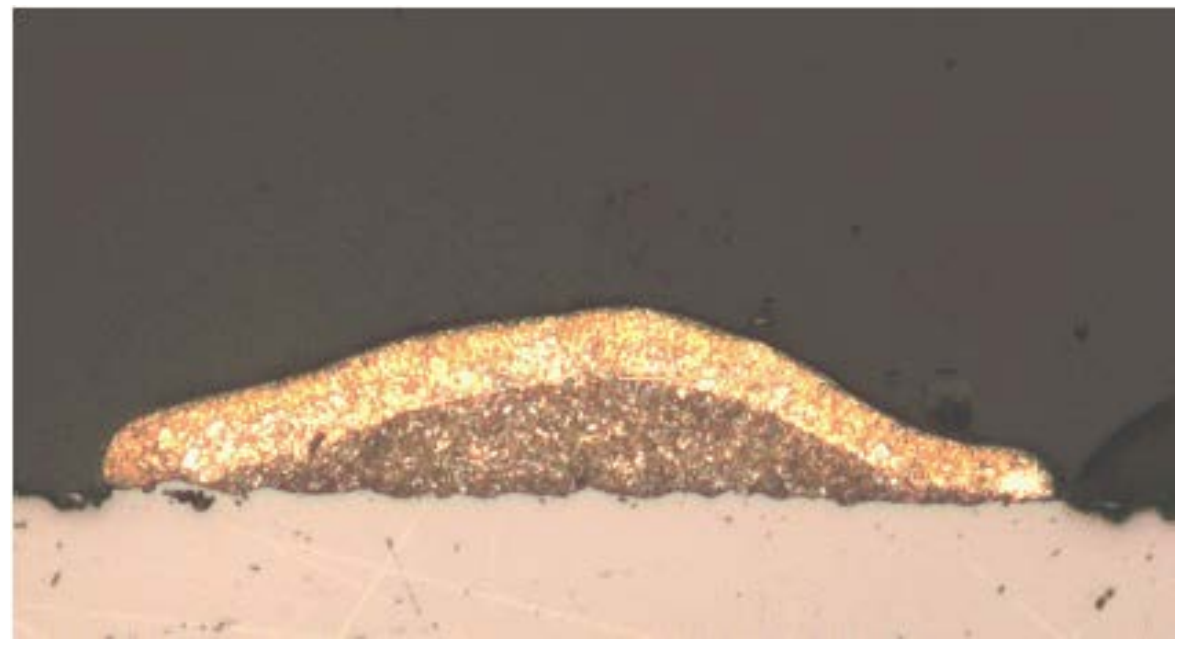

Figure 2.15: SEM image of the cross section of screen-printed contact by applying LIP process [32].

Compared to the common electroplating processes, the LIP method provides an efficient way for depositing a very uniform silver layer. With this method, is achieved an enhancement of $0.3-0.4 \%$ absolute in the cell efficiency on both mono- and multicrystalline screen-printed solar cells [32]. Table 2.2 lists a comparison of geometrical and electrical parameters of the finger defined by screen-printed (SP), photolithographically defined contacts and laser grooved buried contact (LGBC). 


\begin{tabular}{|c|c|c|c|}
\hline Pechniques & Screen printing & $\begin{array}{c}\text { Photolithograph } \\
\text { ical defined } \\
\text { contact }\end{array}$ & $\begin{array}{c}\text { Laser grooved } \\
\text { buried contact }\end{array}$ \\
\hline Parameters & & & \\
\hline finger width & $30-150 \mu \mathrm{m}$ & $2-30 \mu \mathrm{m}$ & $50 \mu \mathrm{m}$ \\
\hline finger height & $10-15 \mu \mathrm{m}$ & $8-15 \mu \mathrm{m}$ & 2.5 \\
\hline finger separation & $0.1-0.26$ & $0.3-0.5$ & $1.25 \mathrm{~mm}$ \\
\hline Finger resistivity & $3.0-3.5 \cdot 10^{-8} \Omega \cdot \mathrm{m}$ & $1.6-1.9 \cdot 10^{-8} \Omega \cdot \mathrm{m}$ & $3.0 \cdot 10^{-8} \Omega \cdot \mathrm{m}$ \\
\hline Contact resistivity & $>1 \mathrm{~m} \Omega \cdot \mathrm{cm}^{2}$ & $<0.3 \mathrm{~m} \Omega \cdot \mathrm{cm}^{2}$ & $3 \mu \Omega \cdot \mathrm{cm}^{2}$ \\
\hline Fill factor & $0.74-0.77$ & $0.81-0.82$ & $0.78-0.79$ \\
\hline Max Cell efficiency & $21.2 \%$ & $24.7 \%$ & $20.6 \%$ \\
\hline Process complexity & $1 \mathrm{~mm}$ & $\mathrm{high}$ & $\mathrm{middle}$ \\
\hline
\end{tabular}

Table 2.2: Comparisons of geometrical and electrical parameters of the finger defined by screen-printed (SP), photolithographically defined contacts and laser grooved buried contact (LGBC).

\subsubsection{Mechanical structure}

The mechanical structure is a patterning method for depositing the etching paste onto the wafer surface. By applying the paste onto a grid pattern designed over a mechanical structure and contacting it with the AR side of the solar cell, the paste is printed and heated by the mechanical structure after the transfer for functionalization. The temperature is controlled at $390^{\circ} \mathrm{C}$ for $90 \mathrm{~s}$. During the heating step, the pastes etches the AR coating layer and the residues left on the surface are removed by deionized water and an ultrasonic bath. 


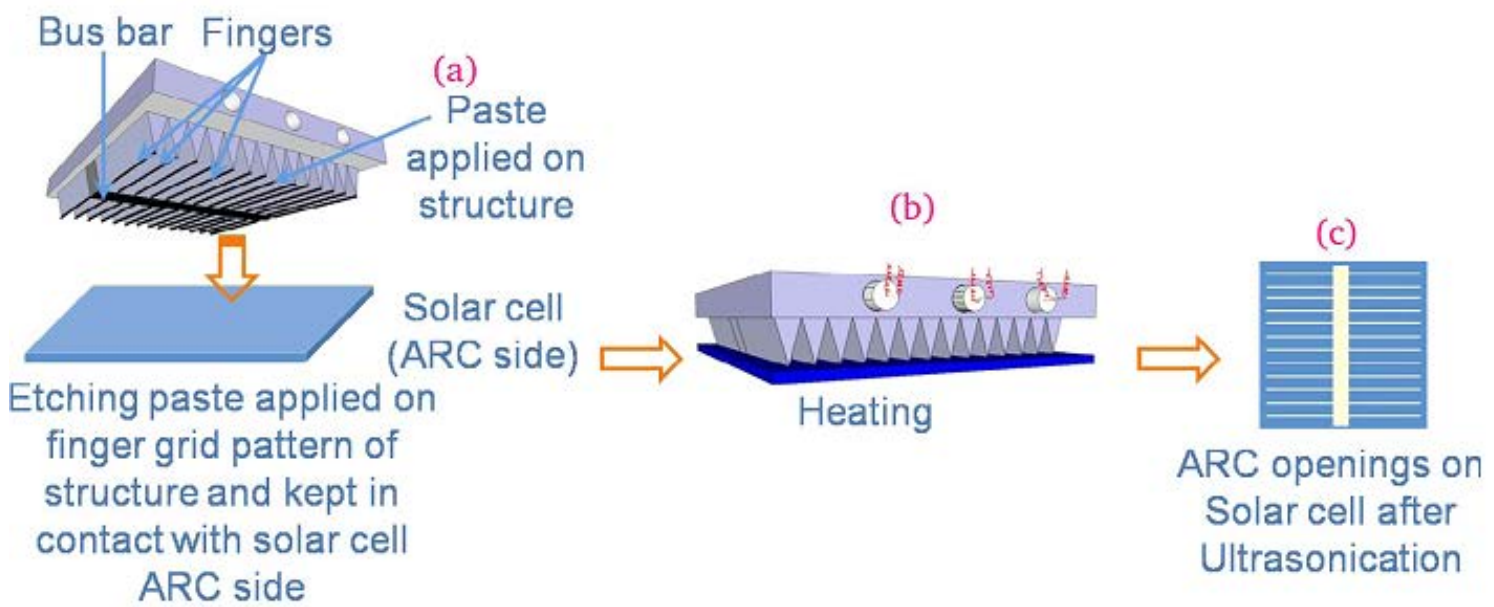

Figure 2.16: Schematic of the mechanical structure method using etching paste [27].

\subsubsection{Pad printing}

The pad-printing is an economical method for front side metallization which has been studied over years at Fraunhofer ISE since 1998 [33]. The process stars with spreading inks by squeegee (doctor blade) from the paste reservoir over an etched surface of the photopolymer plate. By moving the blade back, the redundant pastes on the surface are retracted to the reservoir. As the paste evaporates, the surface become sticky, then the pad moves down and upwards on the surface and the pastes are stack to the surface from the etched area. The next step is to move away the plate revealing the substrate underneath and move the pad downward again onto the substrate sticking the paste to the surface of wafer. In the final step the pad is lift up and the paste is released from it. The process is illustrated in Fig. 2.17. 

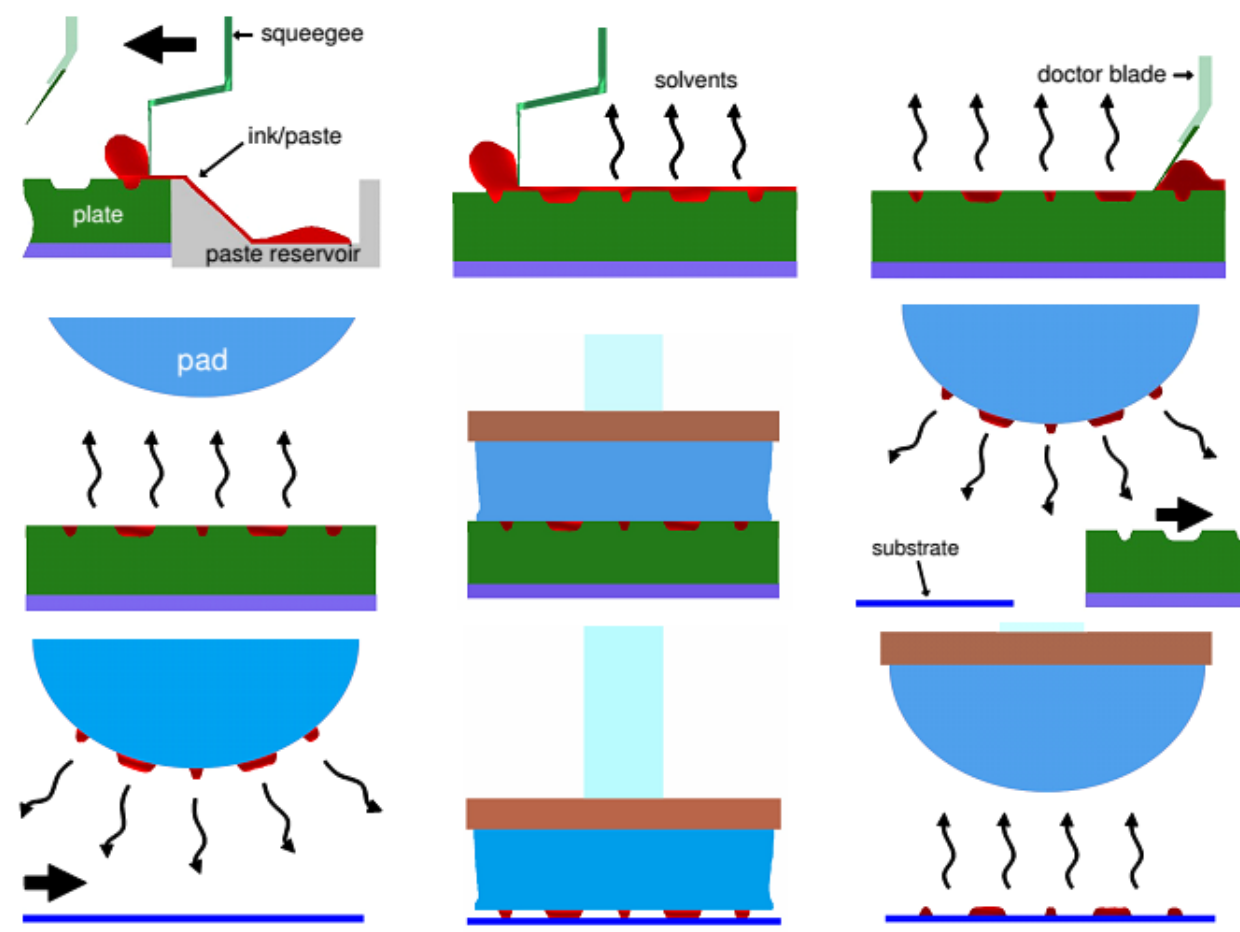

Figure 2.17: Schematic of the pad printing process [32].

In this process, the paste has a similar composition as the screen printing ones with slightly lower viscosity and a resin is added for increasing the adhesion. This transfer padprinting process has shown a technological and economical advantage for solar cell metallization. However, considering an industrial mass production, the rotary padprinting machine can be used instead for the higher throughput rate.

\subsubsection{Laser micro-sintering}

The laser micro-sintering (LMS) technique is a non-contact way for the front grid metallization since during the layer-by-layer process the finger line is directly formed on the substrate by laser-fused powder material of micron size without any mechanical contact of the deposition equipment. As Fig. 2.18 illustrated, a thin metal powder is spread on the AR layer in a grid pattern and by scanning the laser beam over it, the metal particles are sintered and adhered to the AR coating surface due to the condensation of the powder caused by immediate expansion and non-equilibrium pressure from the 
melting. And the formed sintered layer is then thickened by a light-induced plating process [33].

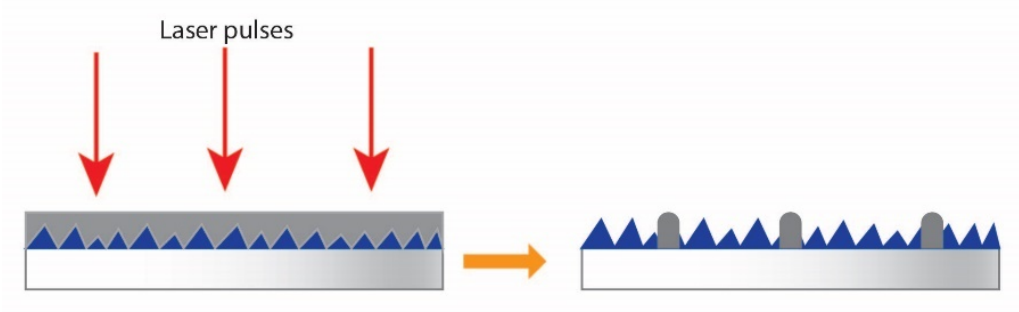

Figure 2.18: Schematic of laser micro-sintering process.

Almán et al. [33] has reported a solar cell with LMS technique achieving $14.5 \%$ cell efficiency. Very fine "seed" layer with only $15 \mu \mathrm{m}$ width is produced using tungsten powders. The difficulty of this technique is to find proper laser processing parameters for melt and sinter the metal powder without damaging the underlying thin doped emitter.

\subsubsection{Ink-jet printing}

The nozzle printing technology provides a non-contact and direct-write way for highdefinition line deposition independent of substrate morphology. The line width with $<20$ $\mu \mathrm{m}$ [34] even < $1 \mu \mathrm{m}$ [35] can be achieved by using this technology which is ultra-thin compared to the minimum line width obtained by screen printing. Ink-jet technology was originated and extensively developed in the 1950's for the desktop inkjet printer. The first application using ink-jet technology for solar cell metallization was done by Teng et al. in 1988 [36] and it is still under investigation and development by several institute such as the National Renewable Energy Laboratory (NREL) for improving the printing resolution and quality.

Ink-jet can be classified into two types: continuous ink jet (CIJ) and drop-on-demand (DOD). The latter mode has been developed for metallizing solar cells using both metalorganic and nanoparticle inks [36-38]. The DOD system uses a piezoelectric transducer, which can generate on-demand droplet that are ejected through a nozzle toward the 
substrate surface. While the CIJ system ejects a stream of droplets, which are charged by an electrode and using a high voltage plate can be directed onto substrate, while the waste droplets are deflected into a gutter for the recirculation (see Fig. 2.19).

(a)

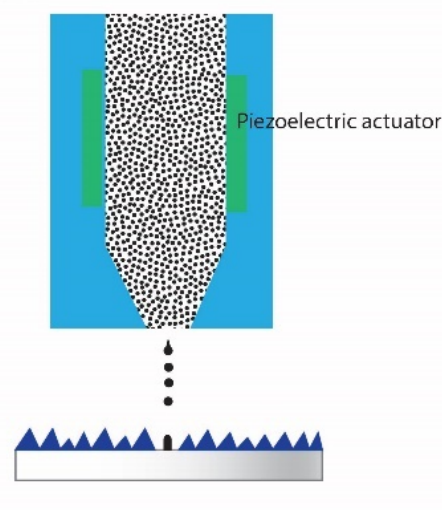

(b) Piezoelectric crystal

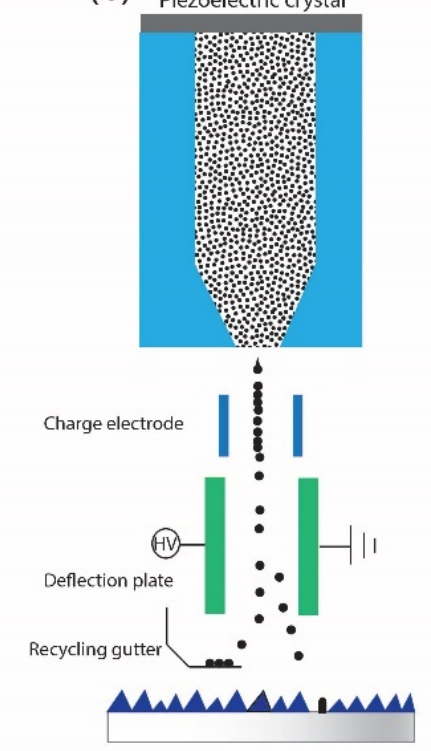

Figure 2.19: Schematic of inkjet printing system (a) drop-on-demand system with piezoelectric actuator (DOD) (b) continuous stream ink-jet system (CIJ). Adapted from [33].

The drawback of inkjet printing system is limited to low aspect ratio due to the low viscosity of the ink in a single-pass printing. Thus, a subsequent plating step of multiplepass printing is needed to thicken the finger line. The geometric requirement of the finger line is compromised with the electrical and mechanical properties. The printing speed is also constrained to tens of $\mathrm{mm} / \mathrm{s}$ owing to the parallel nature of the ink jet, which affects a lot the industrial throughput.

\subsubsection{Aerosol jet printing}

Like the inkjet printing, the metal aerosol jet printing is also a nozzle based printing technique developed by Optomec Inc., USA. And both techniques have shown a significant advantage over the conventional metallization deposition on silicon and thin 
film solar cells [38]. Instead of directly printing metal-containing ink, the ink is atomized to aerosol jet pneumatically or ultrasonically, entrained in a gas stream and then transported to a heating tube and nozzle, which is the key part of the printer (see Fig. 2.20).

The inks used in pneumatic and ultrasonic atomizer differ from each other mainly due to the maximum particle size. The ultrasonic atomizer adopts metal organic and nano particle inks which have a particle size smaller than $50 \mathrm{~nm}$ with viscosity between 0.7 and $30 \mathrm{cP}$ while the pneumatic atomizer can be used for particle size up to $500 \mathrm{~nm}$ with viscosity range up to $1000 \mathrm{cP}$. Hence, the pneumatic atomizer can be regarded as a potential candidate for industrial application due to the wide range of ink selection.
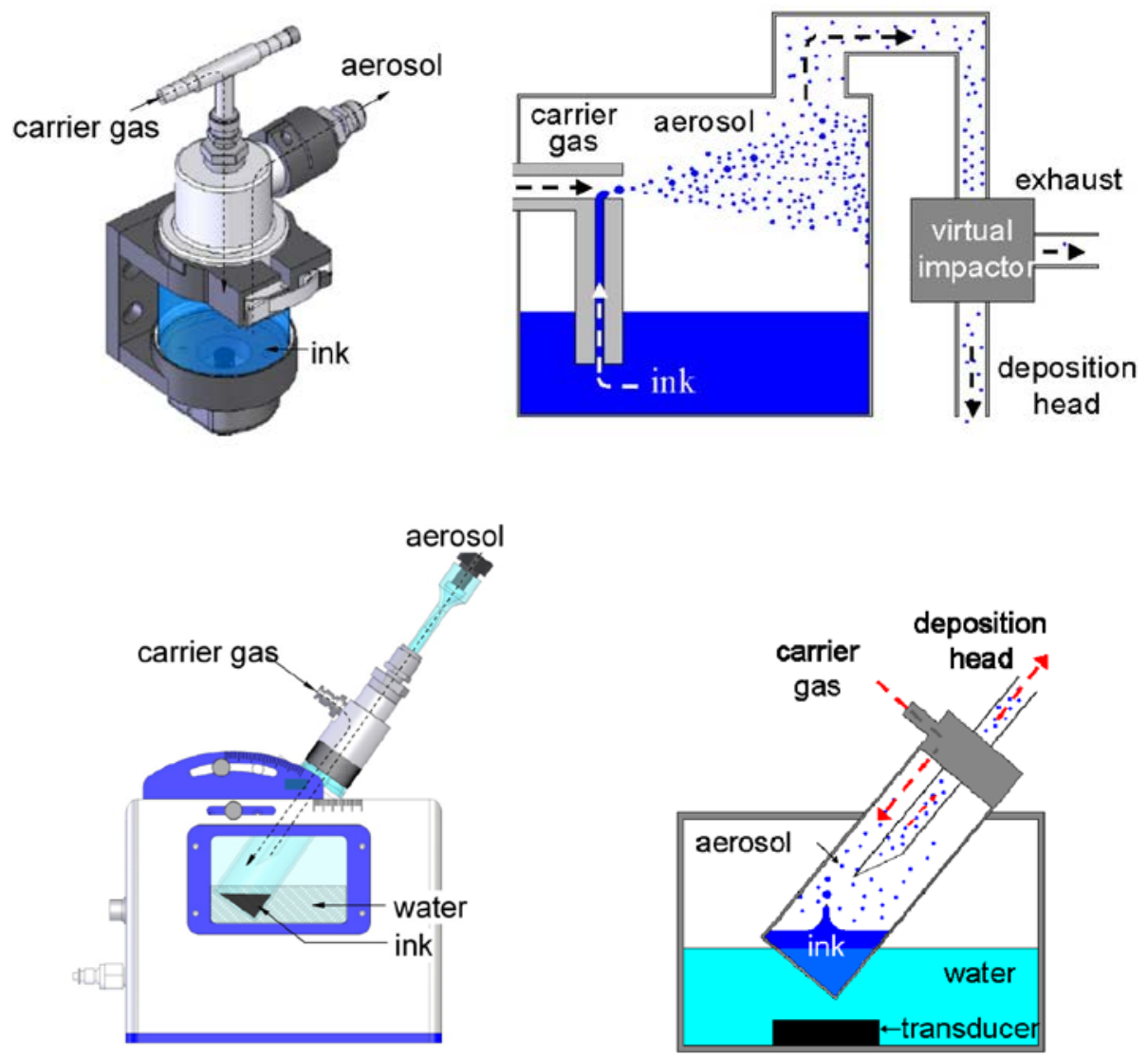

Figure 2.20: Schematic of the working principle of pneumatic and ultrasonic atomizer [32]. 


\subsubsection{E-jet printing}

Electrohydrodynamic jet printing (E-jet printing) technique aims at constructing ultrafine electrodes (<10 $\mu \mathrm{m}$ width) by applying a high electric field between the nozzle and a conducting support substrate, which generates an electro-hydrodynamic effect that pulls out the silver paste from the small nozzle forming a Taylor cone shape [39]. The nozzle opening diameter plays a critical role in the high-resolution printing process. Park JangUng et al. demonstrated the ability of printing lines with width as small as $700 \mathrm{~nm}$ using a nozzle with an internal diameter between 0.3 and $30 \mu \mathrm{m}$ [39]. Nonetheless, the silver paste used in this method lacks yield stress, which helps to construct high-aspect ratio electrodes by preventing the paste from spreading and collapsing.

\subsubsection{Dispensing printing}

The dispensing printing technique has been explored as a single-pass and non-contact printing techniques. It has the capability of printing high viscous silver paste, which forms high-aspect-ratio electrodes. Fig. 2.21 illustrates the principle of dispensing printing. The silver paste rapidly recovers in yield stress at a zero strain rate after pushed on the substrate through a tip, and stops spreading reaching the final shape. In the dispensing process, the distance between tip and substrate needs to be controlled precisely and the particle size should be less than seven times the nozzle tip's diameter.

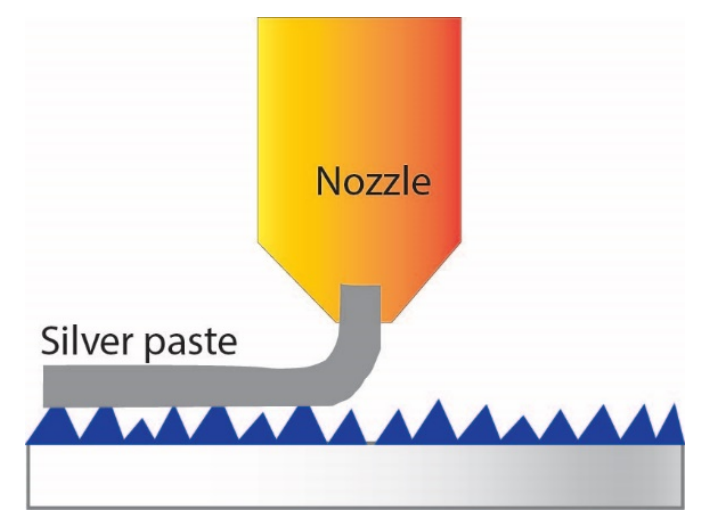

Figure 2.21: Schematic of principle of dispensing printing. 
The conventional time-pressure dispenser can print the high yield stress silver paste. However, when the paste gets contacted with the textured surface of a crystalline silicon solar cell, the paste stops wetting the surface, i.e. it cannot fully flow into the texturized gap to contact with the substrate. Therefore, the mechanical interlocking will be reduced.

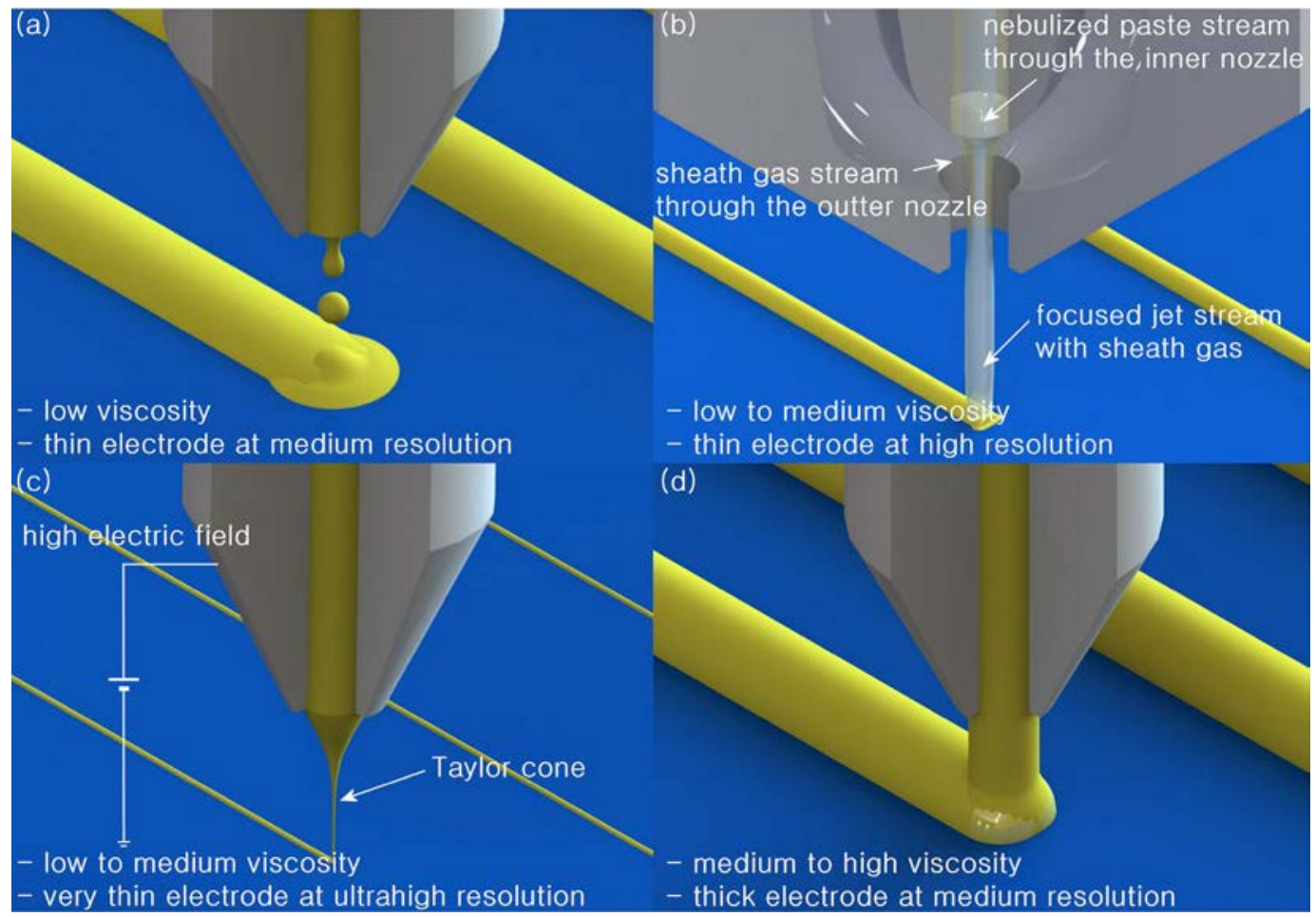

Figure 2.22: Schematic of four principle nozzle-based printing techniques [3].

\begin{tabular}{|c|c|c|c|c|}
\hline $\begin{array}{r}\text { Printing } \\
\text { Techniques }\end{array}$ & $\begin{array}{c}\text { Inkjet } \\
\text { printing }\end{array}$ & $\begin{array}{c}\text { Metal aerosol } \\
\text { jet printing }\end{array}$ & E-jet printing & $\begin{array}{c}\text { Dispensing } \\
\text { printing }\end{array}$ \\
\hline Parameters & $<200 \mathrm{~nm}$ & $\begin{array}{c}<50 \mathrm{~nm} \text { or } \\
<500 \mathrm{~nm}\end{array}$ & $100 \mathrm{~nm}$ & $<10 \mu \mathrm{m}$ \\
\hline Minimum finger & $34 \mu \mathrm{m}$ & $66 \mu \mathrm{m}$ & $30 \mu \mathrm{m}$ & $30 \mu \mathrm{m}$ \\
\hline
\end{tabular}




\begin{tabular}{|c|c|c|c|c|}
\hline $\begin{array}{c}\text { Maximum finger } \\
\text { height }\end{array}$ & $3 \mu \mathrm{m}$ & $22 \mu \mathrm{m}$ & $34 \mu \mathrm{m}$ & $34 \mu \mathrm{m}$ \\
\hline Aspect ratio & $<0.1$ & 0.33 & 0.79 & 0.79 \\
\hline Contact resistivity & & & $0.98 \mathrm{~m} \Omega \cdot \mathrm{cm}^{2}$ & \\
\hline Fill factor & 0.72 & 0.81 & & \\
\hline Cell efficiency & $19.7 \%$ & $18.3 \%$ & $17.2 \%$ & $17.2 \%$ \\
\hline
\end{tabular}

Table 2.3: Comparison of four nozzle-based printing techniques applied metallization on solar cell.

Hence, a new printing technique called electrostatic-force-assisted dispensing is proposed by D Y. Shin et al. aiming at producing fine line with high aspect ratio. This technique combines both characteristics and advantages of dispensing and E-jet printing techniques by using a nozzle enabling printing a highly viscous silver paste and applying an electric filed between the nozzle and the crystalline silicone solar cell substrate (see Table. 2.3). In addition, a 2 wt\% fluorosurfactanct is added into the silver paste which reduces the surface energy for wetting the substrate surface but simultaneously decreases the adhesion force. By applying this method, the printed electrodes stay firmly to the textured antireflection layer surface after the co-firing process. The electrostaticforce-assisted dispensing printing technique produced electrodes with a width as thin as $30.4 \mu \mathrm{m}$ and an aspect ratio of 0.79 . The contact resistivity of the electrodes was $0.98 \pm$ $0.92 \mathrm{~m} \Omega \cdot \mathrm{cm}^{2}[3]$. 


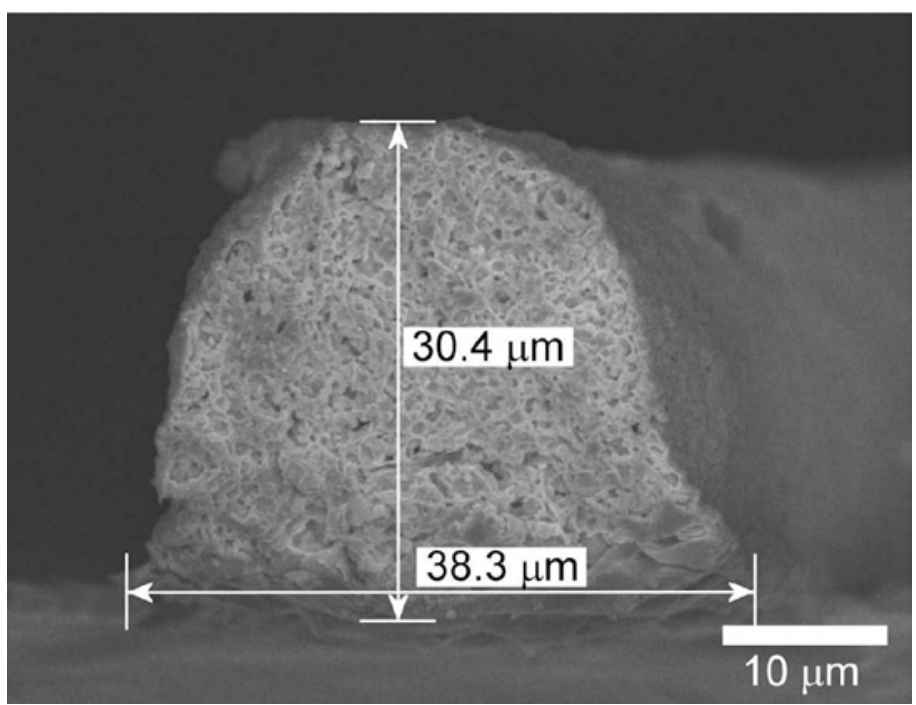

Figure 2.23: Cross sectional SEM image of the electrode printing by electrostatic-forceassisted dispensing printing technique with a high aspect ratio of 0.79 [3].

Table 2.24 shows a comparison of all front contact metallization techniques. It can be seen that although photolithographical defined contact provide the highest cell efficiency the process sequence is also the most expensive and complex. Nozzle-based metallization techniques can provide high cell efficiency with direct-write method. However, the materials that can be printed using small-orifice nozzles are limited by the particle size and viscosity to avoid clogging. LIFT metallization method can print both solid material like metal films and metallic ink and paste without special preparation. This amplify the choices of the metallization material and the results shows that LIFT conductive metallic line can get very high aspect ratio and therefore obtain a high cell efficiency for the solar cell. 


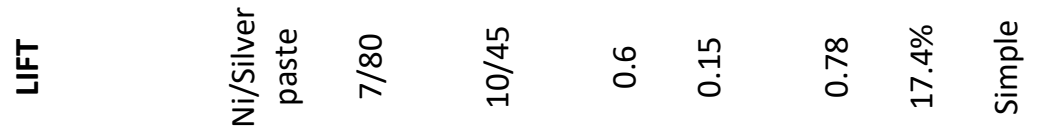

空

离芯芯

端言紊

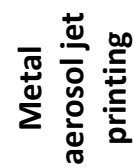

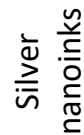

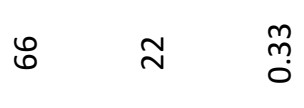

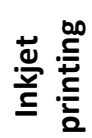

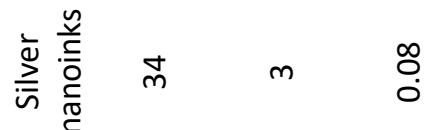

$\stackrel{0}{0}$

$\begin{array}{lll}-1 & \stackrel{0}{\infty} & \frac{0}{0} \\ 0 & \stackrel{\infty}{\rightarrow} & \text { i }\end{array}$

ঠ்

$\stackrel{2}{\stackrel{2}{\circ}} \stackrel{\frac{0}{\circ}}{\stackrel{2}{\xi}}$

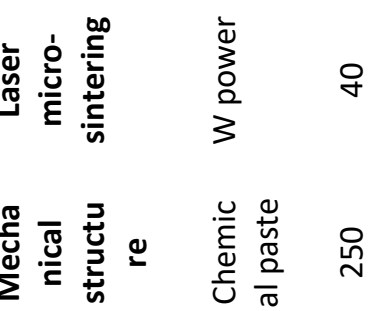

离

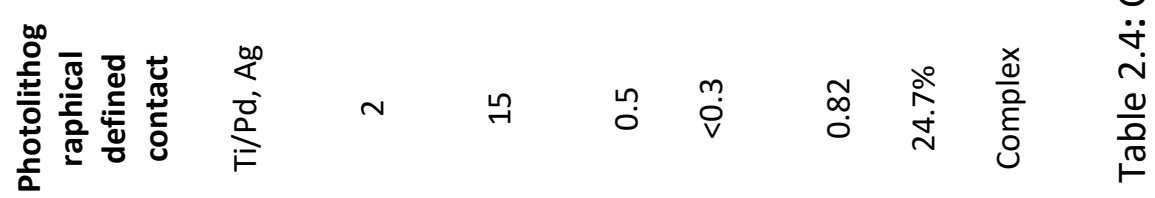

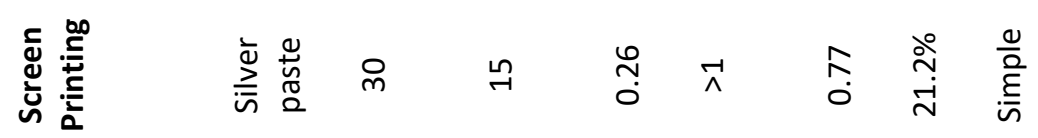

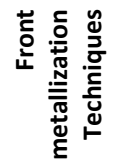

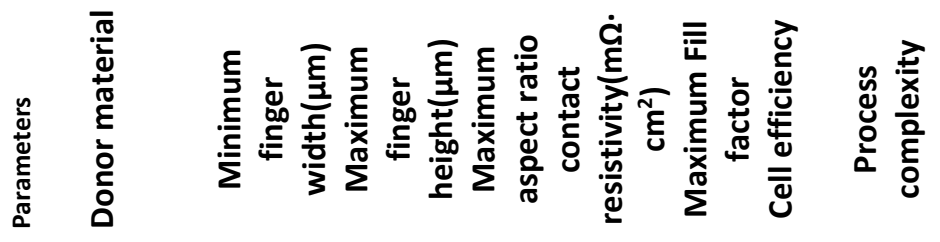




\section{Laser-induced forward transfer (LIFT)}

Laser-induced forward transfer (LIFT) is a direct-write laser technique, capable of transferring a variety of materials (especially metallic solid materials or material dissolved in an assisting matrix) in different sizes onto a number of substrates [7].

In principle, a portion of thin film material coated on a non-absorbing, transparent carrier substrate is propelled by the laser-generated vapor formed on the interface on which the laser beam is focused, and ejected from the donor to an acceptor substrate which is placed underneath, in close proximity to the film (see Fig. 3.1) [8].

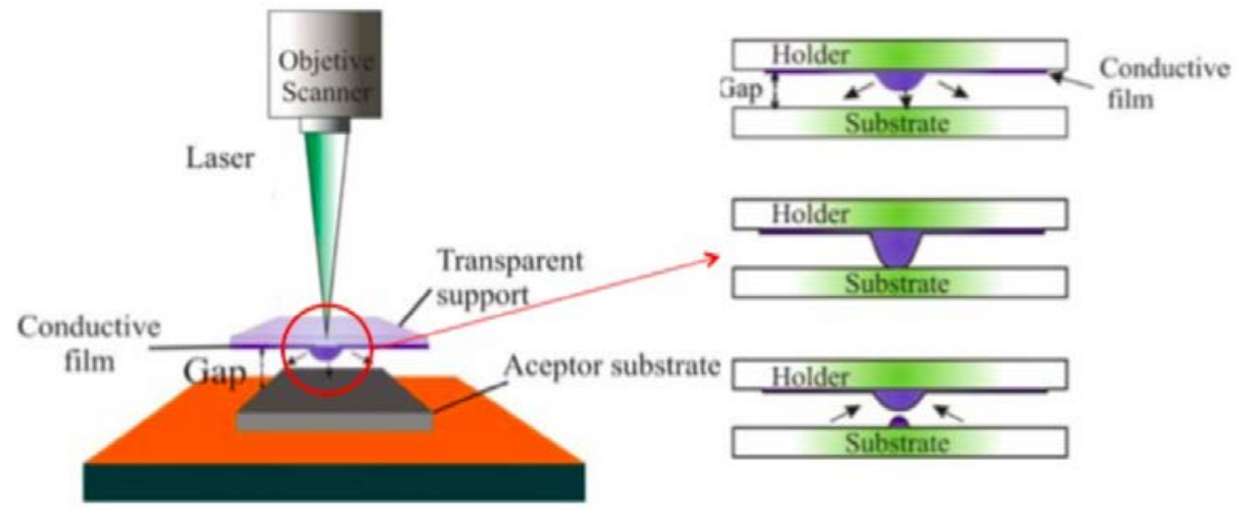

Figure 3.1: Schematic of the traditional LIFT process and its working principle [13].

The LIFT comprises a complex laser-matter interaction mechanism, which involves energy conversion and phase transitions. Therefore, it is difficult to describe the process using fundamental models. Diverse process parameters, mainly laser parameter such as wavelength, pulse shape, pulse duration, focal spot size, pulse energy (or fluence), affect the final transferred process. Apart from the laser parameters, the donor-acceptor mechanism also play an important role in the transferred voxel formation, such as film thickness, roughness, gap distance between donor and substrate. For a successful, smooth and high-resolution deposition, process parameters of LIFT need to be specifically controlled and optimized.

Comparing to the previously mentioned nozzle-based techniques in chapter 2 like screen printing, inkjet printing, dispensing, Aerojet printing and E-jet printing, LIFT provides a non-contact, nozzle-free, direct-write and mask-free method for transferring 
complex materials, which has no special limitations and can be directly formed from the transfer process [8].

High-resolution patterns can be directly printed for the fabrication of microstructure without consumption of the expensive photoresist layer and a sequence of complex and numerous wet-etching processing steps which are required in the photolithography technique.

\subsection{LIFT characterization}

The characterization of LIFT processes has been done by two main methods. One is the morphological study by optical microscopy, confocal microscopy or atomic force microscopy etc. of the transferred films on the acceptor and film on the donor as a function of the process conditions including laser parameters, material properties and gap distance.

The other method for characterizing the LIFT process is by using the imaging system. One imaging system is to use the high-speed video for recording consecutive images of the LIFT process. The schematic diagram of the apparatus used to the high-speed video is illustrated in Fig. 3.2. By this method, the complete view of the LIFT process can be recorded by the video camera [40].

The other imaging system is made for obtaining the time-resolved images from a side view by strobing a flash lamp after a specified time delay to the laser pulse, set by a digital delay generator. The detail of this setup will be given in the next chapter. 


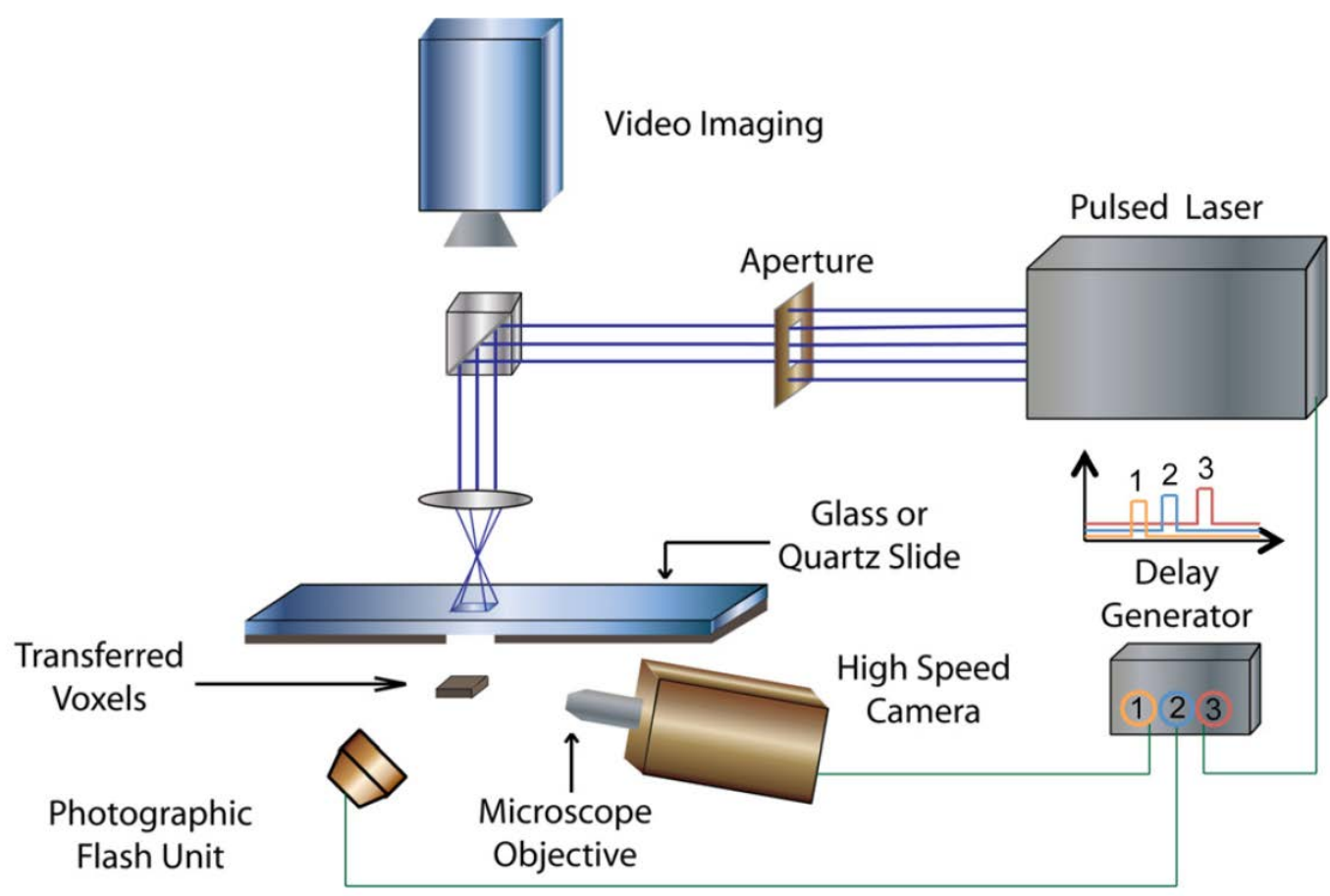

Figure 3.2: Schematic diagram of the system used to capture high-speed video images [40].

\subsection{Different LIFT techniques}

In the basic LIFT set-up, the laser directly irradiates on the donor film, heats and vaporize the material in rapid, resulting in a high-pressure vapor cavity. However, there are some modified LIFT methods depending on the type of absorption of laser and transfer the energy into mechanical dynamic energy for the liquid ejection.

One of the variation of LIFT is the Matrix-Assisted Pulsed Laser Evaporation DirectWrite (MAPLE-DW). In MAPLE-DW, the donor material can be powders, particles or even viable cells, which is enclosed in an evaporable matrix system, like frozen solvents or volatile organic binder, with the suspension or dispersion as transparent carrier coated on the donor substrate. The principle of MAPLE-DW is illustrated in Fig. 3.3. 


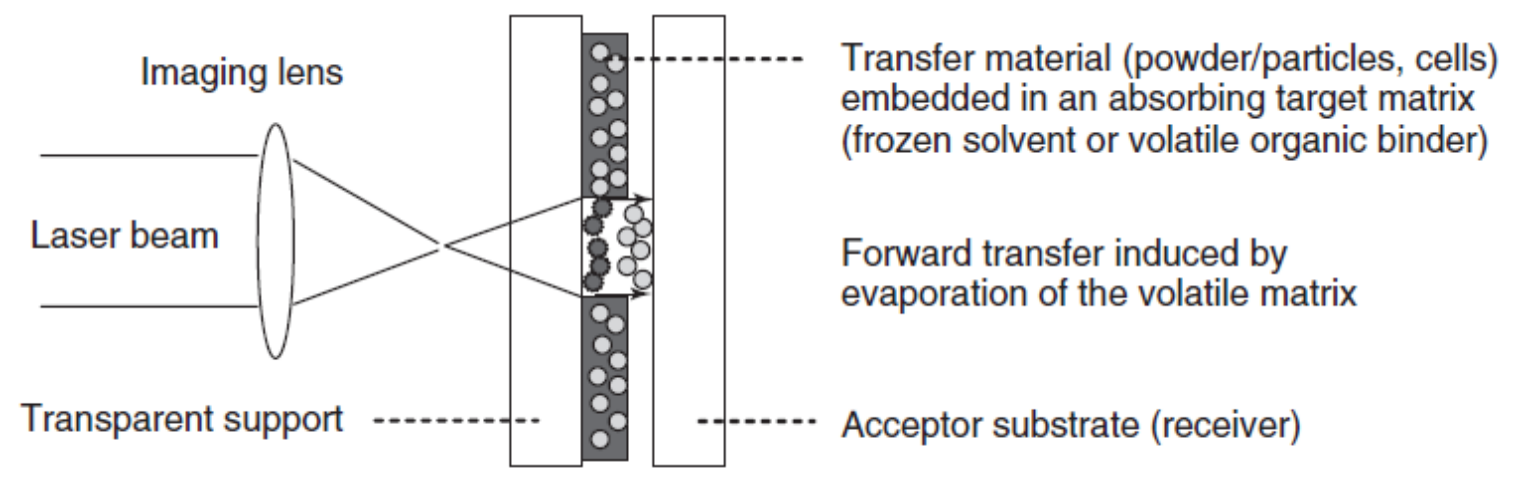

Figure 3.3: Working principle of Matrix-Assisted Pulsed Laser Evaporation Direct-Write (MAPLE-DW) [8].

In MAPLE-DW process, the laser irradiation is absorbed by the volatile components of the matrix, which is vaporized to form the pressure for propel the transfer material to the acceptor. This method was originally developed for rapidly prototype mesoscopic passive electronic device components, like resistor and capacitors [41-43]. However, the "liquid transfer vehicle" as matrix makes MAPLE-DW a promising method for biogenic material transfer applied in the fabrication of biodevices and sensors. ArF excimer lasers were employed in the process since most organic compound absorb in the short UV range [44].
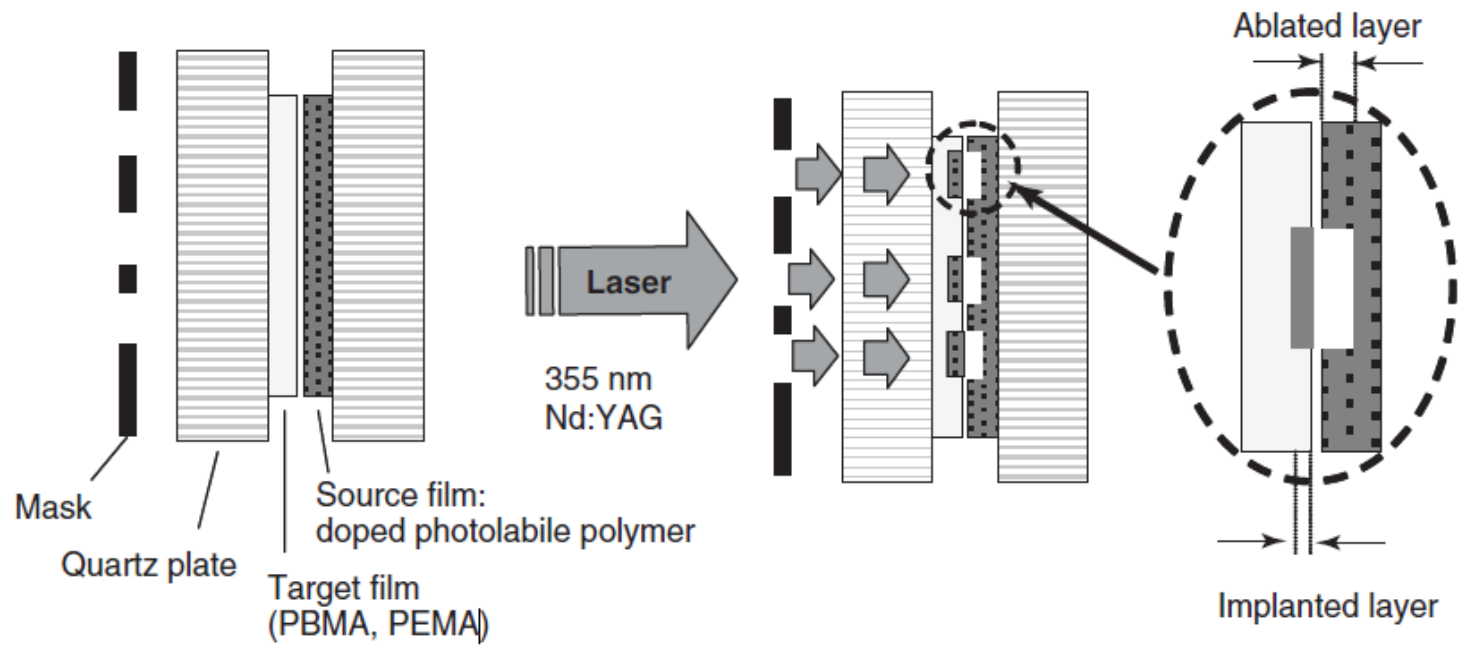

Implanted layer

Figure 3.4: Working principle of laser molecular implantation (LMI) poly (butyl methacrylate, poly (ethyl methacrylate) [8]. 
Laser Molecular Implantation (LMI) is a technique for laser transfer of molecular compounds. In principle, a laser beam passes a nonabsorbing polymer layer through the transparent substrate, irradiating an absorbing polymer film consisting of molecules, such as pyrene [45] or photochromic molecules [46] is put in contact with the target film (see Fig. 3.4). The absorbing polymer matrix is decomposed by the laser beam into small fragments which implants the embedded probe molecules into the nonabsorbing polymer layer. The donor substrate must be a glass with high transparency and low glass transition temperature, since the pulse laser induced phase transition makes a controlled softening of the target film and makes the implantation more efficient.

As one of the most important variations of the LIFT, dynamic release layer-LIFT (DRLLIFT) involves an intermediary, laser-absorbing layer for shielding the donor material from direct interaction with laser beam (see Fig. 3.5). This prevents photolytic and pyrolytic damage to the transfer material and enables printing of many sensitive materials. The DRL layer can be metal film like Al film, gold films, titanium film or $\mathrm{Cr}$ films [47]. Thin polymer film has been also as an absorbing layer for transfer damage-free inks without contamination of the polymer in Arnold' group [48]. They investigate on a so-called blister-actuated laser-induced forward transfer process (BA-LIFT). BA-LIFT involves the rapidly expanding, sealed blister which can deform the polymer film and drive flow in the surround fluid. The trapped hot gases cool and keep trapped inside the blister cavity, therefore it is isolated from the transfer material.

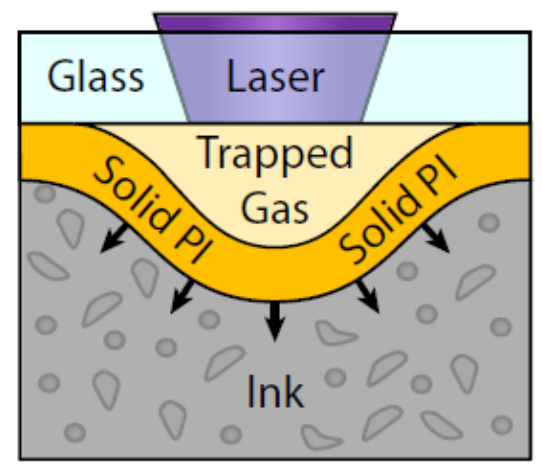

Figure 3.5: Scheme of blister-actuated laser-induced forward transfer process (BA-LIFT) [48]. 
As LIFT process involves mainly two kinds of material: solid and liquid. LIFT process can be classified depending on its donor film material.

\subsubsection{LIFT of solid materials}

For solid thin film deposition, LIFT can transfer both inorganic materials such as metal, oxide, ceramics etc. and organic matters such as polymer, carbon nanotubes and biomaterials etc. This solvent-free (or "dry") direct-write deposition method has been developed for the fabrication of microdevices with versatile functional materials.

For instance, the LIFT micro-sized metal pattern allows the electrical conducting path for electronic circuits. Bohandy performed the first attempt of metal deposition using single ns pulses (15 ns pulse length) of a UV excimer laser (ArF, $193 \mathrm{~nm}$ wavelength). The donor used was a fused silica carrier coated with $400 \mathrm{~nm}$-thick metal film. And the gap distance was set at several micrometers. This setup allowed them to print, in a highvacuum chamber environment, copper and silver lines with a width of 50 and $15 \mu \mathrm{m}$ respectively. This study was regarded as the first systematic LIFT transfer study and application for direct-write process of conductive line in electronic devices. Kantor et al. studied the LIFT deposition of high melting point metal films like $\mathrm{Ti}, \mathrm{Cr}$ and $\mathrm{W}$ on glass by using a ruby laser (694 nm, $20 \mathrm{~ns}$ pulse duration) [49]. The results demonstrated that the film removal mechanism was a thermomechanical blow off in the solid phase that was affected by the solid-solid poor adherence (see Fig. 3.6). Other LIFT-deposited metals like aluminum [50], nickel [51], gold [51-53], palladium [54] etc. have also been investigated by various groups. 


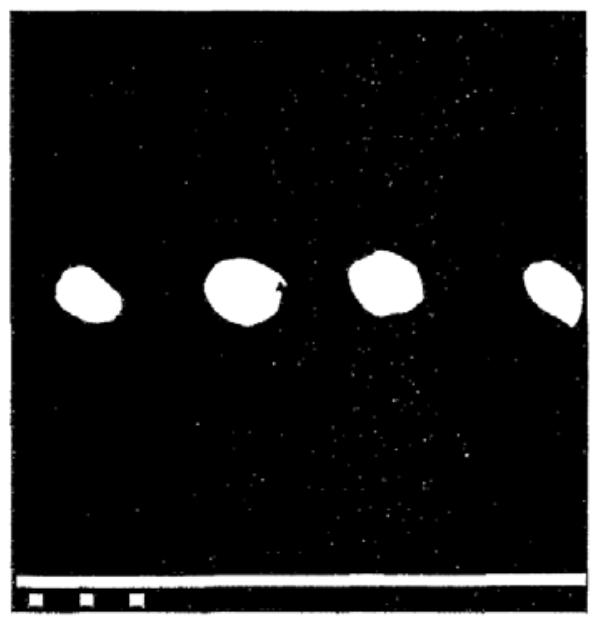

Figure 3.6: Backscattered electron images of transferred thin tungsten pattern. The scaling bar indicates $100 \mu \mathrm{m}$ [49].

Recently Claas W.Visser et al. manufactured a pure-metal pillar by stacking micrometer-sized disk-shaped copper and gold drops using a ps laser (515 nm, 6.7ps) [55]. This enable the 3D metal printing by LIFT for constructing high-aspect ratio and high resolution microstructure (see Fig. 3.7).

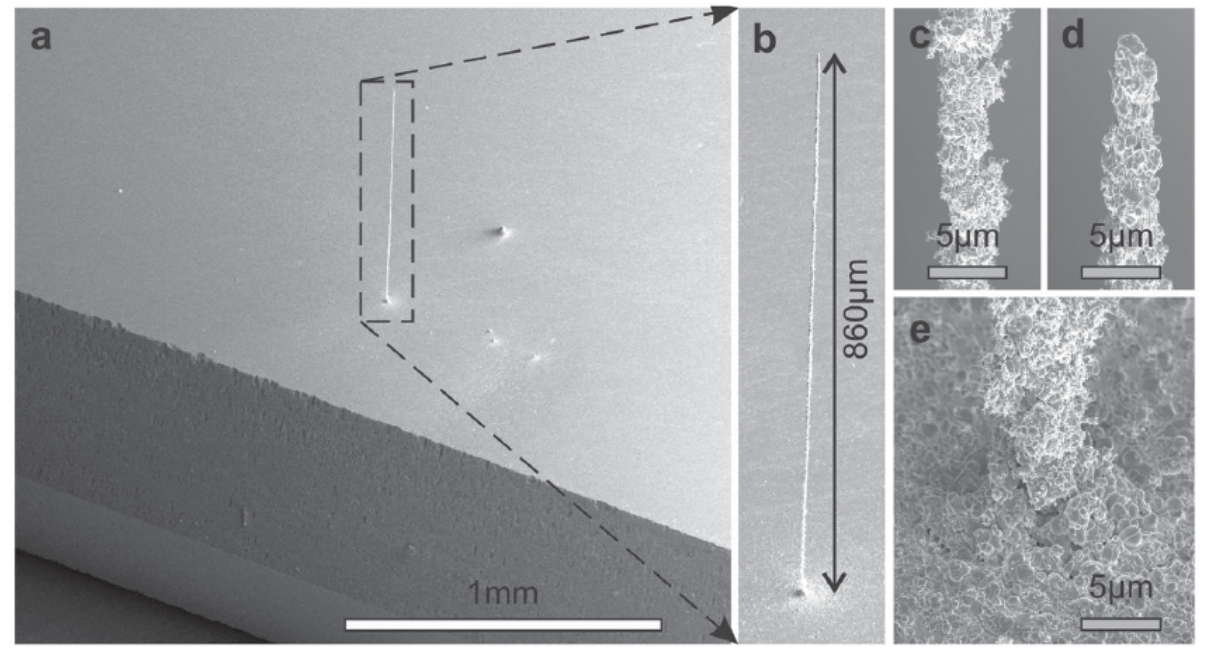

Figure 3.7: High-aspect ratio examples of copper LIFT. a) $860 \mu \mathrm{m}$ long pillar close to the clearly visible edge of a microscope slide. b) Close-up of the pillar. Zooming in further shows that the pillar has a virtually homogeneous thickness: the center d) and top e) are around $4 \mu \mathrm{m}$ thick whereas the bottom diameter (e) is around $6 \mu \mathrm{m}$, due to drops landing next to the pillar [55]. 
For organic materials targeted by LIFT studies, small organic molecules, carbon nanotubes, polymer films have been investigated for developing a microdevice system. In these studies, a sacrificial propellant in the form of a supporting matrix in MatrixAssisted Pulsed Laser Evaporation-Direct Write (MAPLE-DW) or a dynamic release layer $(D R L)$ is often used as a protective material for the active material during transfer. The direct LIFT transfer of conductive polymer PEDOT:PSS of $300 \mathrm{~nm}$ thick film, which is used as an electro-coating material in electronic thin-film devices fabrication, were performed by different laser wavelength by $\mathrm{B}$. Thomas et al. [56]. Owing to the thermal effects and sensitivity of the polymeric materials, it is very difficult to obtain LIFT deposition of polymer films in a direct ablation way without photochemical degradation by a UV radiation or destructive side effect of the physical property like electrical conductivity. However, with a 25 or $50 \mathrm{~nm}$ metallic dynamic release layer (DRL) and two UV laser systems (a $248 \mathrm{~nm} \mathrm{KrF}$ excimer laser with $35 \mathrm{~ns}$ pulse duration and a $355 \mathrm{~nm}$ Nd:YAG with 50 ps pulses), well-defined deposits concerning pixel cohesion, homogeneity and surface roughness can be obtained.

\subsubsection{LIFT of liquid materials}

For the fabrication of electronic micro devices and printed electronics, LIFT provides a direct-write way for printing the conductive metallic interconnection lines by using conductive metallic inks or high viscous nano-paste. By tuning the composition of the ink to an appropriate viscosity, the fluid dynamics of the ejection can be adapted for a highresolution printing of a single-droplet. And continuous patterns can be printed by overlapping of single-droplets. Highly viscous pastes can be directly, homogenously and smoothly transferred.

LIFT can also print with high resolution a wide range of sensitive biomaterials including nucleic acids, proteins, biocells, enzyme, microorganism and DNA for the fabrication of biosensor. Although these biomaterials are solid, we classify them into liquid category since normally the donor film is prepared with the mixture of biomaterials and solutions in suspension status. 
Depending on the rheological behavior, LIFT used liquid donor film can be classified into two categories: Newtonian fluid and non-Newtonian fluid. Table 3.1 summarizes Laser-induced forward transferred of liquid material in the context of Newtonian fluid and non-Newtonian fluid and process parameters used for the studies.

Most of the studies related with LIFT of Newtonian fluid involve the jet formation, which is critical for predicting the material transfer. The jet formation in laser-induced forward transfer is quite different from that in inkjet printing. In the case of laser-induced forward transfer of fluid, such as barium titanate ink [57], water glycerol mixture [16] or bovine serum albumin (BSA) protein solution [58], the transferring process involve the jet formation.

Claudia et.al. use spatial and temporal high-resolved stroboscopic imaging for investigating the liquid jet (alginate-based hydrogel) formation, development of jet and impingement process with collector in the laser-induced forward transfer (LIFT) [59].
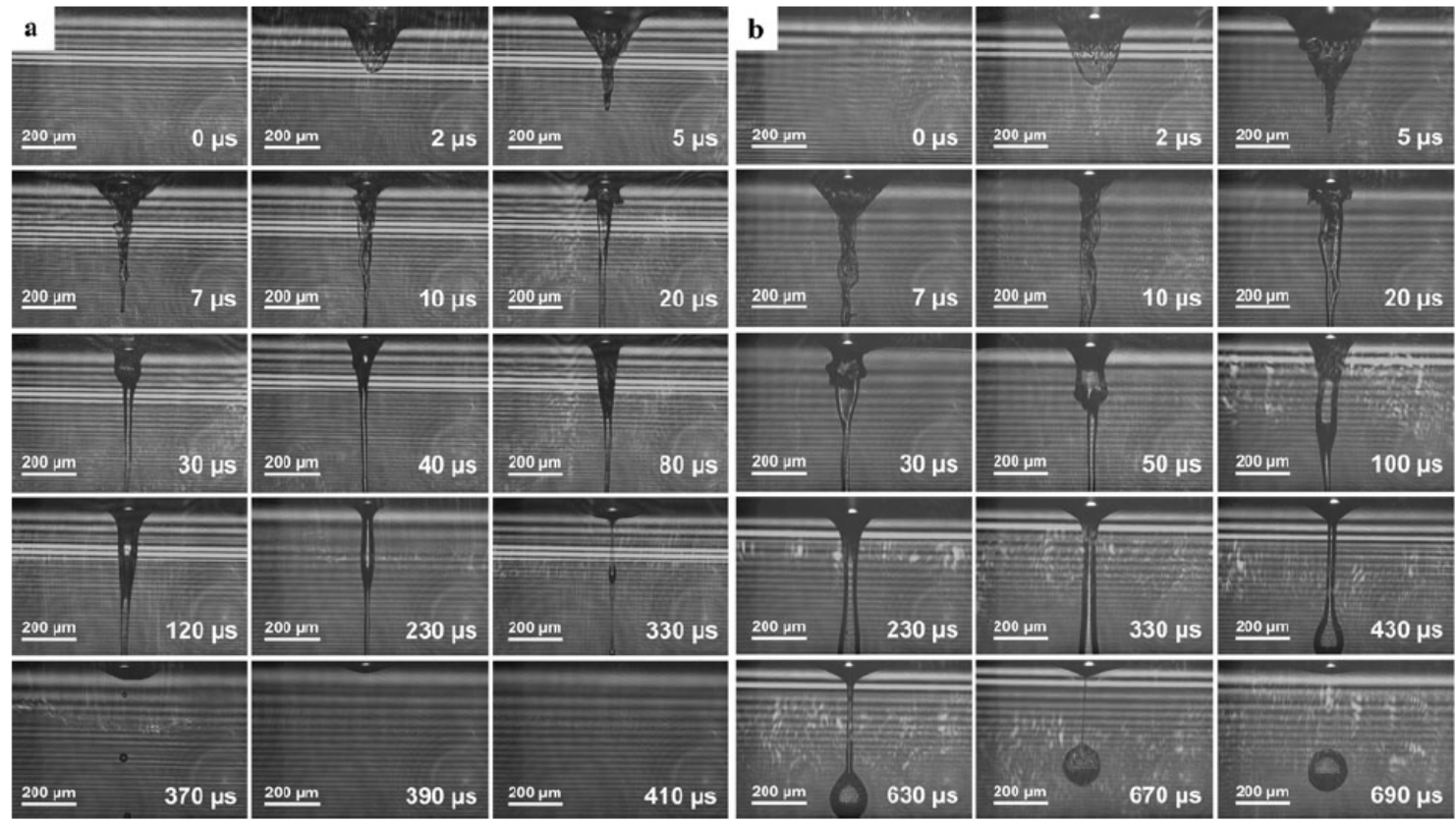

Figure 3.8: Time-resolved images of the hydrogel jet formation during LIFT at two laser fluence (a) $1.6 \mathrm{~J} / \mathrm{cm}^{2}$ and (b) $2.7 \mathrm{~J} / \mathrm{cm}^{2}[59]$. 
The time-resolved image series is shown in Fig. 3.8. It can be observed that there are characteristic transfer stages. And the temporal occurrence and jet formation of the liquid are fluence-dependent. Initially, the laser irradiates on the absorbing release layer (in this case is gold layer) evaporates the local material and forms hot gases on the gold-liquid interface. The liquid absorbs the energy and generates a rapidly expanding vapor pocket blocked inside the liquid (see Fig. 3.9a and Fig. 3.10a). The adjacent liquid shows resistance against the bubble enlargement and results in an elongated bubble. The contact angle of the bubble to the glass slide increase from $90^{\circ}$ until it detaches from the glass slide (Fig. 3.9b-g). When the internal pressure is smaller than the external atmospheric pressure plus surface tension, the bubble keeps expanding outward, increasing in volume but decreasing in pressure. Due to the inertia of the bubble tip moves at a high speed than the rest point of the bubble. At this point, the jet formation is initiated by the inertia, surface tension and the bubble collapse. After the collapsing of the vapor bubble, the lateral liquid with low velocity is pull together.
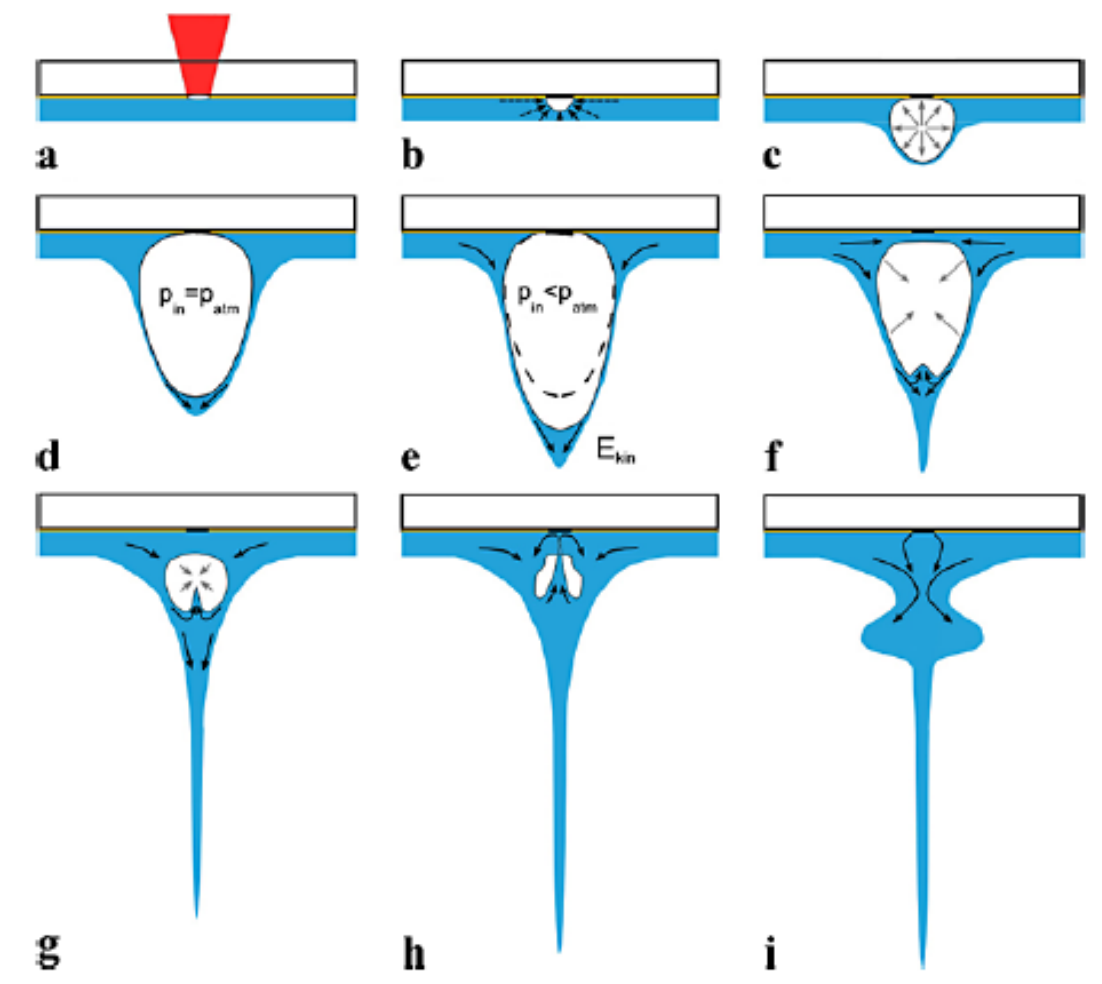

Figure 3.9: Scheme of the bubble dynamics and jet formation in LIFT process [59]. 
This kind of jet formation and development are commonly discussed in several studies $[16,60]$. Despite the difference of absorbing release layer material and its thickness, the ejection dynamics occurs nearly same in spatial and temporal evolution of the features formed, which indicates the same gas-actuated mechanism for the jet formation [59]. For the final application of LIFT technique, the impingement characteristics of the liquid droplet onto solid surface have been widely studied by several groups [61-65]. In general, the critical Weber number $W_{c}$ is applied to indicate the splashing threshold of the liquid. It is given in the expression of the ratio between inertial force and surface tension:

$$
W e_{c}=\frac{\rho v^{2} l}{\sigma}
$$

Where $\rho$ is the liquid density, $v$ is the velocity, $l$ is the characteristic length corresponding to the droplet diameter and $\sigma$ is the surface tension.

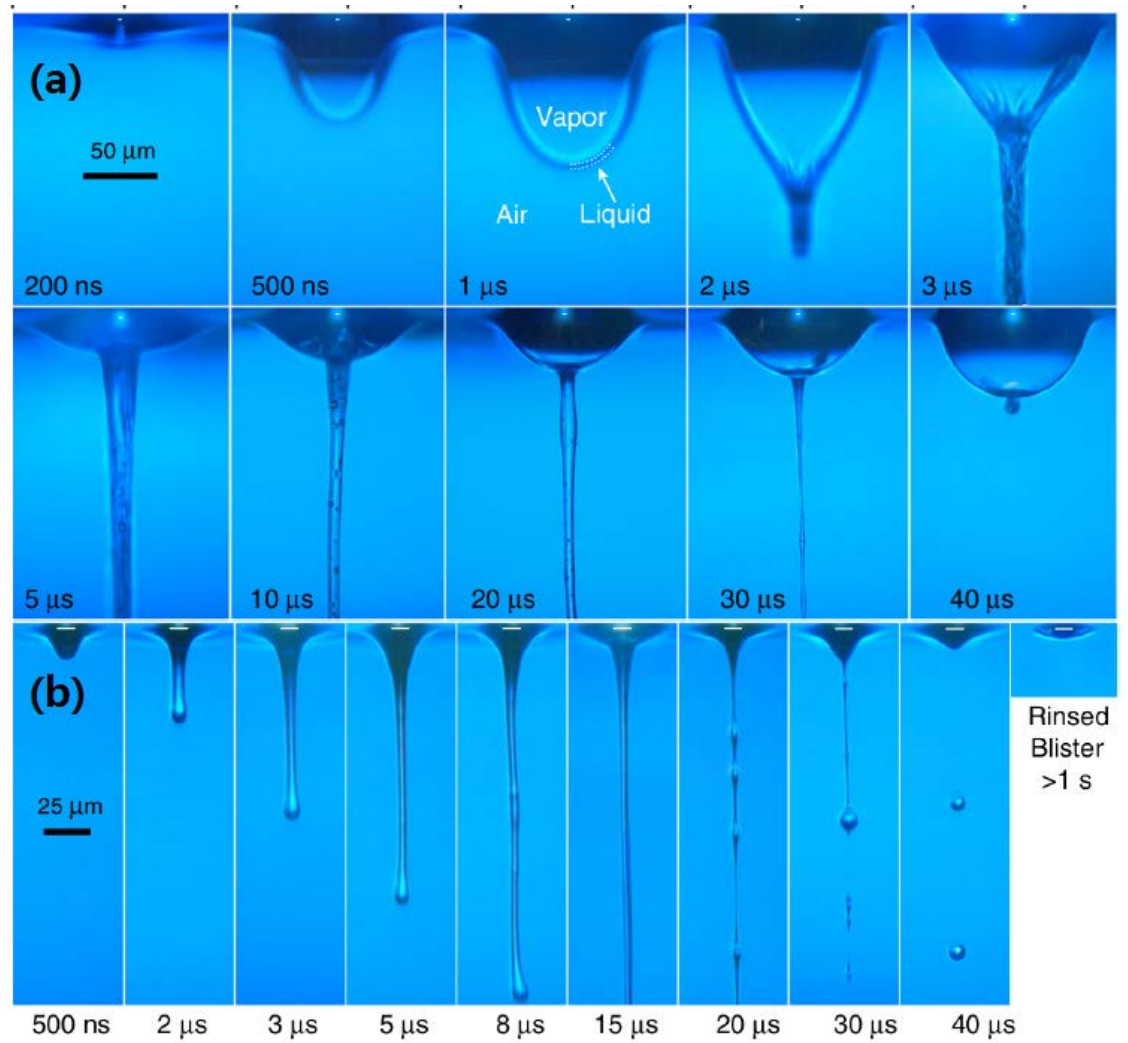

Figure 3.10: Time-resolved images of liquid ejection into ambient air using (a)a 50-nm titanium absorbing layer and (b) a 7- $\mu$ m polyimide absorbing layer [66]. 

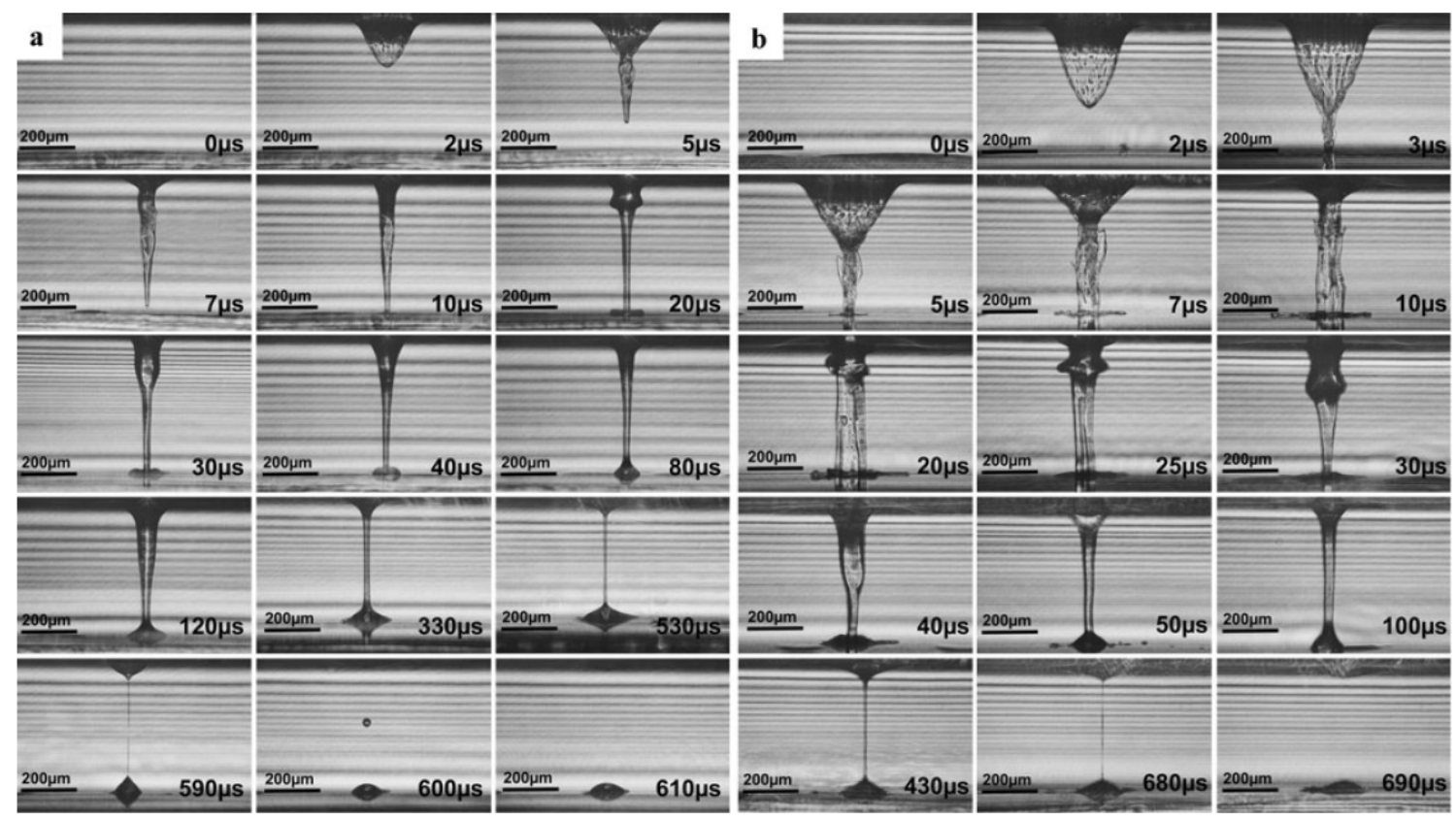

Figure 3.11: Time-resolved images of the hydrogel transfer on the acceptor during LIFT process with two laser influence (a) $1.6 \mathrm{~J} / \mathrm{cm}^{2}$ and (b) $2.7 \mathrm{~J} / \mathrm{cm}^{2}$. The gap distance between donor and acceptor is around $450 \mu \mathrm{m}$ [59].

Weber number indicates that with low impact velocities the droplet spreads on the solid surface, while with higher velocities the droplet initiate splashing and satellite droplet are generated (see Fig. 3.10). However, the droplet size over time not only depends on the inertial forces and surface tension but on wettability and viscosity as well.

Duocastella et. al performed a systematic study of the main LIFT process parameters by printing nanoparticle suspensions, including laser pulse energy, laser beam dimension, gap distance for the understanding of the parameters in the printing process [67]. It has been found that the LIFT process is very sensitive to laser fluence and gap separation. The spot-like transfer or well-defined voxel can be obtained within a narrow fluence range with small gap distance.

When LIFT is applied to high viscosity paste, the voxel can be printed with controllable size and shape by the laser pulse. This process is referred to the laser decal transfer (LDT), which allows the thin-film-like structure for a wide range of applications. 
Mathews et. al. studied the behavior of fluid by high-speed video camera from low viscosity silver nano-particle inks (Newtonian fluid with viscosity $<1 \mathrm{mPa} \cdot \mathrm{s}$ ) to very high viscosity silver nanopaste (non-Newtonian fluid with viscosity $>500 \mathrm{~Pa} \cdot \mathrm{s}$ ) using LDT process [68].

It is observed in the study that the fluid behavior in the low viscosity and very high viscosity regimes are consistent with the results previously reported DRL-LIFT technique [60] with water glycerol mixture. At low viscosity range $(<1 \mathrm{mPa} \cdot \mathrm{s})$, the transfer voxel has three regimes depending on the bubble and jet formation as a function of laser fluence. And the transferred voxel lost the detail of the distribution of the laser pulse due to the surface tension of the ink (see Fig. 3.12a, b).
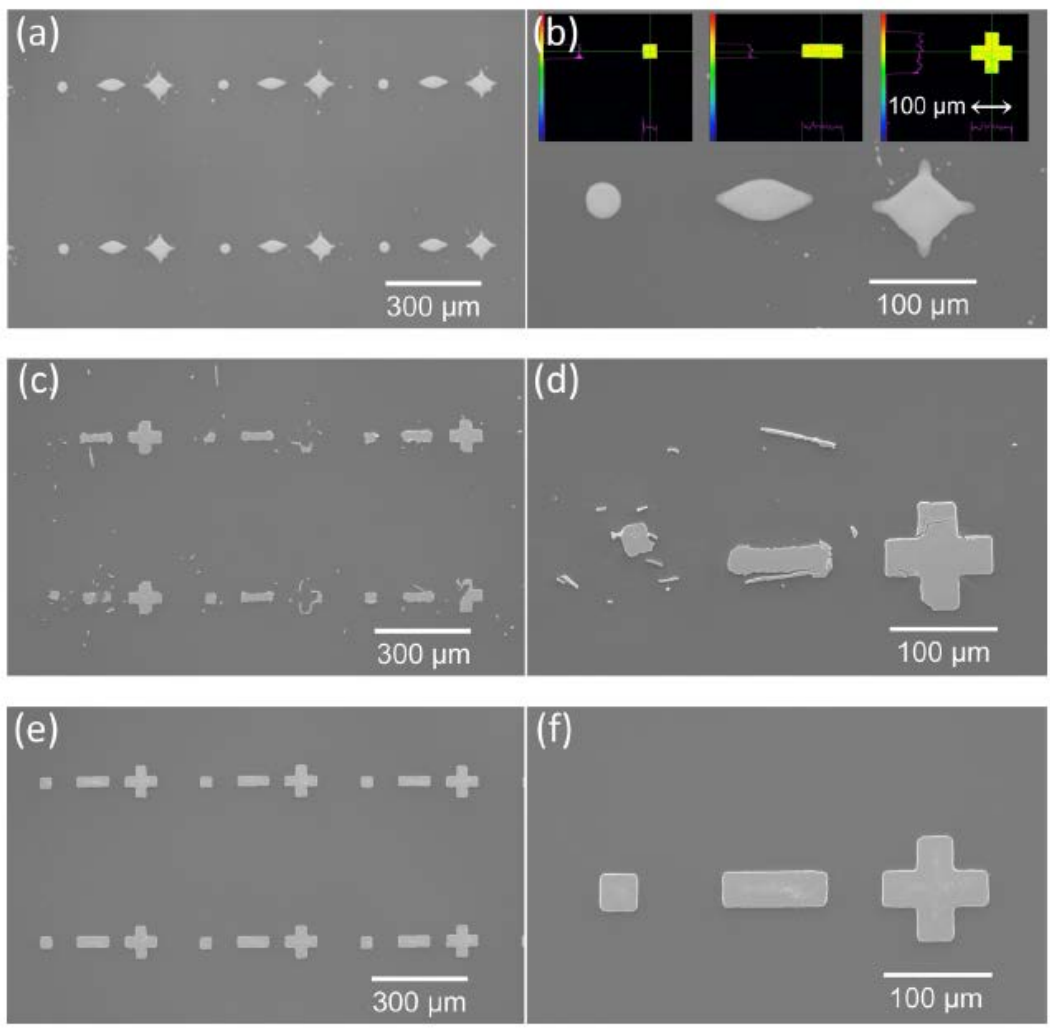

Figure 3.12: SEM images of transferred silver nano-suspensions with different viscosities on Si acceptor substrate. (a, b) Transferred voxel with low viscosity silver nano-inks. (c, d) Transferred voxels with very high viscosity silver nanopastes. $(e, f)$ Transferred voxels with high viscosity silver nanopastes [68]. 
While when using very high viscosities paste (>500 Pa.s), which is a non-Newtonian fluid, the transferred voxel exhibits a solid-like behavior which consistent with the previous studies for DRL-LIFT of metal films [40]. As seen in Fig. 3.12c, d, although the transferred voxel can maintain the shape of the laser pulse, the laser fluence need to separate the voxel from the donor brings about the fracture of the voxel, which is related with the fragmentation and explosion in the transfer (see Fig. 3.13b).

For a high viscosity paste, which has a limited range between 90 and $150 \mathrm{~Pa} \cdot \mathrm{s}$, the laser decal transfer (LDT) process can be performed successfully. The transferred voxels are concrete and congruent with the shape of laser pulse. The voxel rotates during the transfer process when it detaches from the donor substrate (see Fig. 3.12c). This indicates that a successful congruent transfer of the voxel needs a reduced gap distance, i.e. placing the acceptor substrate in close proximity to the donor substrate for avoiding the rotation.

This study concludes several advantages of the LDT process. The LDT doesn't need a dynamic release layer, which avoids the contamination of the residue from the DRL material. And the high viscosity paste can be transferred with well-formed pattern avoiding the surface wetting and the resolution loss. And the voxels transfer at a low speed on the order of $1 \mathrm{~m} / \mathrm{s}$, which reduces the aerodynamic forces and minimizing rotation of the voxel. In addition, LDT allows the congruent transfer of voxel with size from micro to millimeter. However, the process also has a limitation of aspect ratio $1<0.2$ for a congruent transfer). And it has a requirement of high uniformity of the donor ink layer and the spatial uniformity of the laser pulse. 

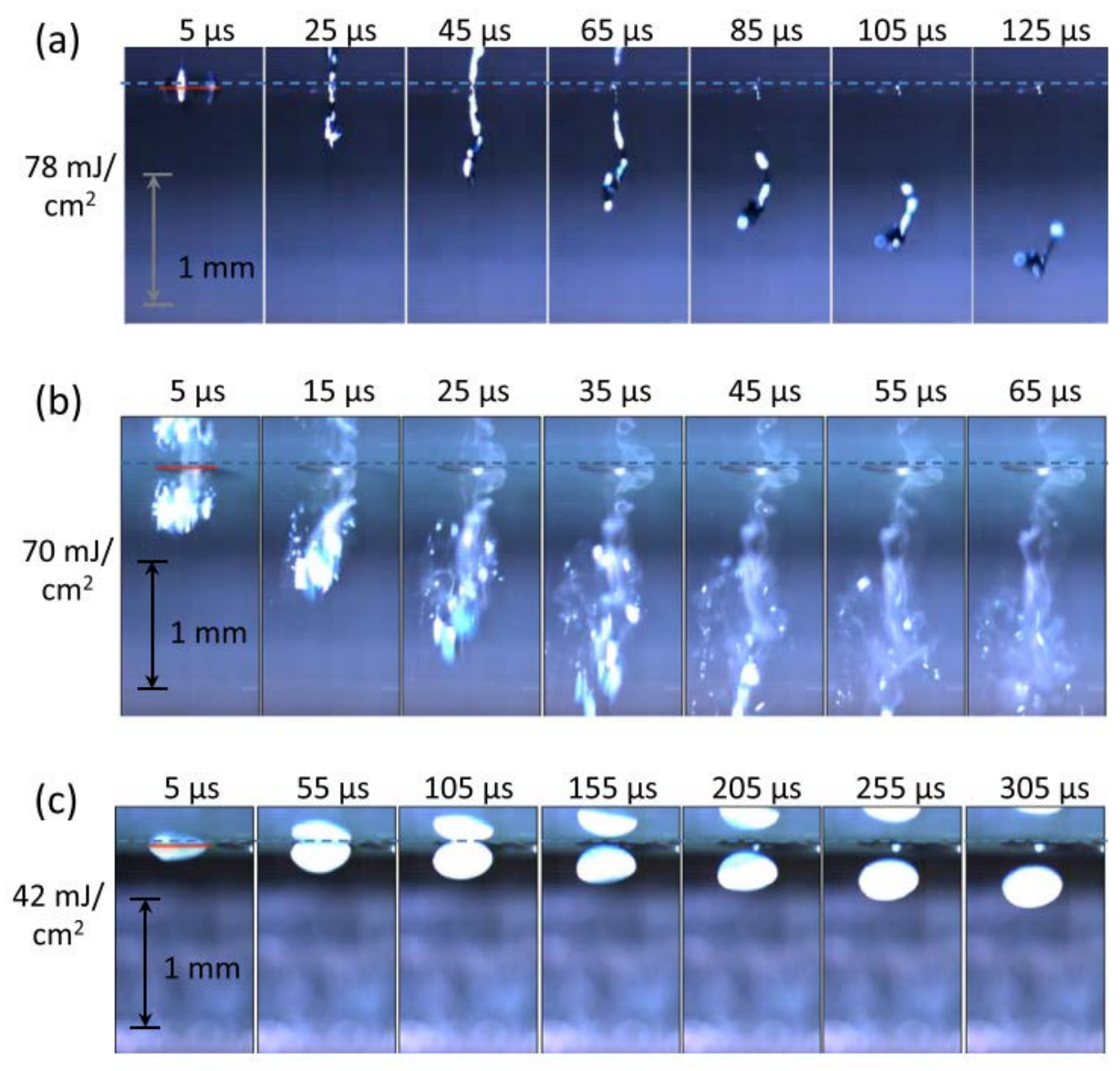

Figure 3.13: Still frame images obtained from the high-speed video (a) Evolution of the jet formation characteristic with low viscosity silver nano-inks. (b) Voxel transfer characteristic with very high viscosity silver nanopastes. (c) Voxel transfer characteristic with high viscosity silver nanopastes [40].

\subsection{LIFT applications}

The feasibility for printing a wide variety of materials has made the LIFT technique capable for diverse application. LIFT has been used to print materials as diverse as DNA for sensors [69], ITO nanoparticles for thin film solar cells [70], organic semiconductors for organic light emitting diodes (OLEDs) [71], bis-pyrene compound for organic think film transistors (OTFT) [72], organic polymer and silver thin films for transducer or sensing device [73], amorphous silicon films for silicon heterojunction solar cell [74], congruent silver 
nanopaste transfer for double split-ring resonator and interconnection for connecting discrete devices in circuits [75] etc.

Attractive features of LIFT process such as easy setup, high flexibility in choice of printing contents and cost-effectiveness even make it feasible for a further industrial implementation. Thus, it is possible to apply LIFT for printing metallic contacts onto flexible optoelectronics devices for the flex/3D electronics industry or for the metallization of the front side of solar cells (fingers and busbars), which also arouses the interest of solar cell researcher and manufacture to adapt this method for the new concept of metallization $[10,76,77]$.

As a key process in the typical solar cell fabrication, the front silver metallization has been probed to improve the efficiency and reduce the cost with competence [27]. Several different printing techniques has been developed to overcome the limitation of the conventional screen-printing $[5,18,37,74,78]$, which is the wel-established and robust method to deposit metal contacts in solar cell industrial production. Compare to the screen-printing technique, LIFT makes it competitive to print a tens of micrometer width of the contact fingers with enhanced aspect radio (height/width), resulting in reducing the shading losses on the cell surface and series resistance but higher sheet resistance and conductivity, which finally leads to an increasing efficiency of solar cell.

The electrical properties like conductivity, contact resistivity of the LIFT line are not only depending on the material property itself but also on the morphology of the line. In the metallization process, LIFT process is mainly in charge of constructing the morphology of the conductive metallic line. The new concept of the metallization in this thesis is to use LIFT of high viscous silver paste combined with a post-procedure of curing/sintering/firing in a two-step way for obtaining high efficient solar cells.

As discussed before, the high performance of the solar cell requires good LIFT lines formation with high aspect ratio and good electrical behavior for front contact metallization. It is not only correlated with the process parameters, but also depends on the paste printability, which is a time-consuming process that corresponds with the paste 
rheology. Like in the screen printing and dispensing techniques, to fulfill the technical printing behavior, the metal paste must feature a thixotropic behavior combined with a high elastic module and fast recovery time of the paste for reducing spreading and slumping on the substrate to achieve better aspect ratio line [79].

In the LIFT process, the silver paste changes its rheological behavior in three steps. When laser irradiates on the donor film a vapor-pocket is generated inside. The laserinduced vapor pressure acts on the surrounding paste and changes the solid regime of the paste. In these regimes, the silver paste deforms and flows with its viscosity rapidly decreased. After the silver paste dropped from the donor film and transferred to the substrate, there is no pressure applied to the silver paste and the paste starts to recover to the solid regime with time. As the yield stress recovers at a zero strain rate the paste finally stops spreading and keep its morphology.

And the thickness of the final deposit of the silver paste is proportional to the yield stress. For a small yield stress, the surface tension effects dominate the stoppage condition. Therefore, for the LIFT line with high aspect ratio, high yield stress of the silver paste is a necessary factor. However, the high yield stress may lead to another problem in the contact formation. Especially it occurs when the silver paste is printed on the textured surface of crystalline solar cell, since the high yield stress prevents the silver paste from wetting the textured surface and consequently the adhesion is decreased [2]. Moreover, the high yield stress paste also shows greater thixotropy since the more energy for the damage the microstructure, the longer the time needed to reform its structure. 


\begin{tabular}{|c|c|c|c|c|c|c|c|c|}
\hline $\begin{array}{r}\text { Donor } \\
\text { liquid } m\end{array}$ & $\begin{array}{l}\text { ilm } \\
\text { terial }\end{array}$ & Component & $\begin{array}{l}\text { Viscosi } \\
\text { ty of } \\
\text { the } \\
\text { fluid(c } \\
\text { P) }\end{array}$ & $\begin{array}{c}\text { Film } \\
\text { thickness( } \\
\mu \mathrm{m})\end{array}$ & $\begin{array}{c}\text { Substr } \\
\text { ate }\end{array}$ & $\begin{array}{l}\text { Mini } \\
\text { mum } \\
\text { featu } \\
\text { re } \\
\text { size( } \\
\mu \mathrm{m}) \\
\end{array}$ & $\begin{array}{c}\text { Laser }(\lambda, \\
\text { pulse } \\
\text { duration } \\
1\end{array}$ & $\begin{array}{l}\text { Refere } \\
\text { nce } \\
\text { numbe } \\
r\end{array}$ \\
\hline Newtoni & $\begin{array}{l}\text { Silver } \\
\text { nano } \\
\text { inks }\end{array}$ & $\begin{array}{l}\text { Silver nano- } \\
\text { particle inks } \\
\text { with }\end{array}$ & $\begin{array}{c}1- \\
50000 \\
0\end{array}$ & $0.5-10$ & $\begin{array}{l}\text { Polish } \\
\text { ed } \\
\text { silicon } \\
\text { wafer }\end{array}$ & 2 & $\begin{array}{c}\mathrm{Nd}: \mathrm{YVO}_{4} \\
\text { laser } \\
(355 \\
\mathrm{nm}, 30 \\
\mathrm{~ns}) \text { and } \\
\mathrm{Nd}: \mathrm{YAG} \\
\text { laser } \\
(266 \\
\mathrm{nm}, 10 \\
\mathrm{~ns}) \\
\end{array}$ & {$[68,80]$} \\
\hline an fluid & $\begin{array}{l}\text { Aque } \\
\text { ous } \\
\text { soluti } \\
\text { on }\end{array}$ & $\begin{array}{l}\text { mixture of } \\
\text { glycerol and } \\
\text { water with } \\
\text { Triton X-100 } \\
\text { surfactant or }\end{array}$ & 7.8 & 5 & $\begin{array}{l}\text { poly- } \\
\text { Llysine- } \\
\text { coated } \\
\text { glass } \\
\text { slide }\end{array}$ & 25 & $\begin{array}{c}\mathrm{Nd}: Y A G \\
\text { laser } \\
\text { (355 } \\
\mathrm{nm}, 10 \\
\mathrm{~ns})\end{array}$ & $\begin{array}{l}{[16,17,} \\
60,66, \\
81,82]\end{array}$ \\
\hline & $\begin{array}{l}\text { Orga } \\
\text { nic } \\
\text { ink(N } \\
\text { MP) }\end{array}$ & $\begin{array}{l}\text { N-methyl-2- } \\
\text { pyrrolidone }\end{array}$ & $\begin{array}{l}0.5- \\
53.8\end{array}$ & $1-10$ & & & $\begin{array}{c}\mathrm{Nd}: Y A G \\
\text { laser } \\
\text { (355 } \\
\mathrm{nm}, 20 \\
\mathrm{~ns})\end{array}$ & [83] \\
\hline & $\begin{array}{l}\text { Silver } \\
\text { nano } \\
\text { paste }\end{array}$ & NPS & $\begin{array}{c}10000 \\
0\end{array}$ & $1-5$ & $\begin{array}{l}\mathrm{Au} / \mathrm{Pol} \\
\text { yimide }\end{array}$ & $<10$ & $\begin{array}{c}\mathrm{Nd}: \mathrm{YVO}_{4} \\
\text { laser } \\
(355 \\
\mathrm{nm}, 30 \\
\mathrm{~ns}) \\
\end{array}$ & {$[11,84]$} \\
\hline $\begin{array}{l}\text { Non- } \\
\text { Newtoni } \\
\text { an fluid }\end{array}$ & $\begin{array}{l}\text { Silver } \\
\text { paste }\end{array}$ & $\begin{array}{l}\text { Silver micro } \\
\text { particles }\end{array}$ & $\begin{array}{c}28000 \\
0- \\
40000 \\
0\end{array}$ & $30-50$ & $\begin{array}{l}\text { Polish } \\
\text { ed } \\
\text { silicon } \\
\text { wafer }\end{array}$ & 100 & $\begin{array}{l}\mathrm{Nd}: Y A G \\
\text { laser } \\
(532 \\
\mathrm{nm}, 20 \\
\mathrm{~ns})\end{array}$ & $\begin{array}{c}{[13,19,} \\
76,85, \\
86]\end{array}$ \\
\hline & $\begin{array}{l}\text { Solde } \\
r \\
\text { paste }\end{array}$ & $\begin{array}{c}\text { type } 6 \text { and type } 7 \\
\mathrm{~Pb} \text {-free solder } \\
\text { pastes with a } \\
\text { composition of } \mathrm{Sn} \\
96.5 / \mathrm{Ag} \\
3.0 / \mathrm{Cu} 0.5 \\
\text { (weight percent) }\end{array}$ & & $10-25$ & $\begin{array}{l}\text { Polish } \\
\text { ed } \\
\text { silicon } \\
\text { wafer }\end{array}$ & 25 & $\begin{array}{c}\text { Nd:YVO } \\
4 \text { laser } \\
\text { (355 } \\
\text { nm,65 } \\
\text { ns) }\end{array}$ & [11] \\
\hline
\end{tabular}

Table 3.1: Laser-induced forward transferred liquid material and parameters used for the studies. 


\section{Experimental and characterization method}

\subsection{Laser Systems}

Four DPSS lasers have been employed in the experimental work. In the LIFT study, a 532 $\mathrm{nm}$ nanosecond laser (Explore Spectra Physics Explorer) is the most applied one in the ns LIFT process study and front grid fingers and busbar printing on the CIGS solar cell. Two ps-pulsed lasers (Ekspla Atlantic and Onefive Katana) have been used in the work to study the ps LIFT process. The detailed laser system is described as below. CW green laser (Millennia Pro s-Series $15 \mathrm{~S} \mathrm{~J}$, Spectra-Physics) is employed for make curing/sintering experiment. The laser used in the time-resolved imaging system in Princeton University is a frequency-tripled $\mathrm{Nd}: \mathrm{YVO}_{4}$ laser (Coherent AVIA) emitting at $355 \mathrm{~nm}$ with a pulse duration of 20 ns.

\subsection{1 $532 \mathrm{~nm}$ Nanosecond laser set-up}

The system consists of a laser head and a L08 power supply. The laser source used in the system is a diode pumped, ns-pulsed, solid state $\mathrm{Nd}: \mathrm{YVO}_{4}$ laser (Explore Spectra Physics Explorer), emitting at $532 \mathrm{~nm}$ with a pulse duration of $15 \mathrm{~ns}$. The Explore laser performance specifications are listed in Table 4.1.

\begin{tabular}{|c|c|}
\hline \multicolumn{2}{|c|}{ General Characteristics } \\
\hline Wavelength & $532 \mathrm{~nm}$ \\
\hline Output power & $20-150 \mathrm{kHz}$ at $50 \mathrm{kHz}$ \\
\hline Pulse Repetition rate & $<15 \mathrm{~ns}$ at $50 \mathrm{kHz}$ \\
\hline Pulse width (FWHM) & $\mathrm{Nd}: \mathrm{YVO}_{4}$ \\
\hline Gain Medium & \\
\hline \multicolumn{2}{|c|}{ Beam Characteristics } \\
\hline \hline Spatial mode & TEMo0 \\
\hline \hline Quality (M ${ }^{2}$ r & $<1.3$ \\
\hline
\end{tabular}

Table 4.1: Explore laser performance specifications for $532 \mathrm{~nm}$ of wavelength. 
The laser system is operated as a stand-alone system from a PC by a LabView-based graphical user interface (L-Win software) for monitoring and controlling the features of the Explore laser frequency such as laser frequency and pump current, which corresponds to the final pulse energy output. An alternative way to control the output power with more stability is to use a programmable laser beam attenuator (AT4040 OPTEC).

The beam is directed to the working stage by passing a beam expander, three steering mirrors and via an attenuator AT4040, an iris for cutting the outer part of the beam before entering the optical scanner (Scanlab HurrySCAN II) with an f-theta lens ( $250 \mathrm{~mm}$ focal length). Finally, the beam is focused into the interface between glass and silver paste to initiate transfers (see Fig. 4.1). The beam waist at the focus is $12.5 \mu \mathrm{m}$, which has been measured both directly using a beam profiler (Ophir Spiricon) and indirectly by means of the Liu method.

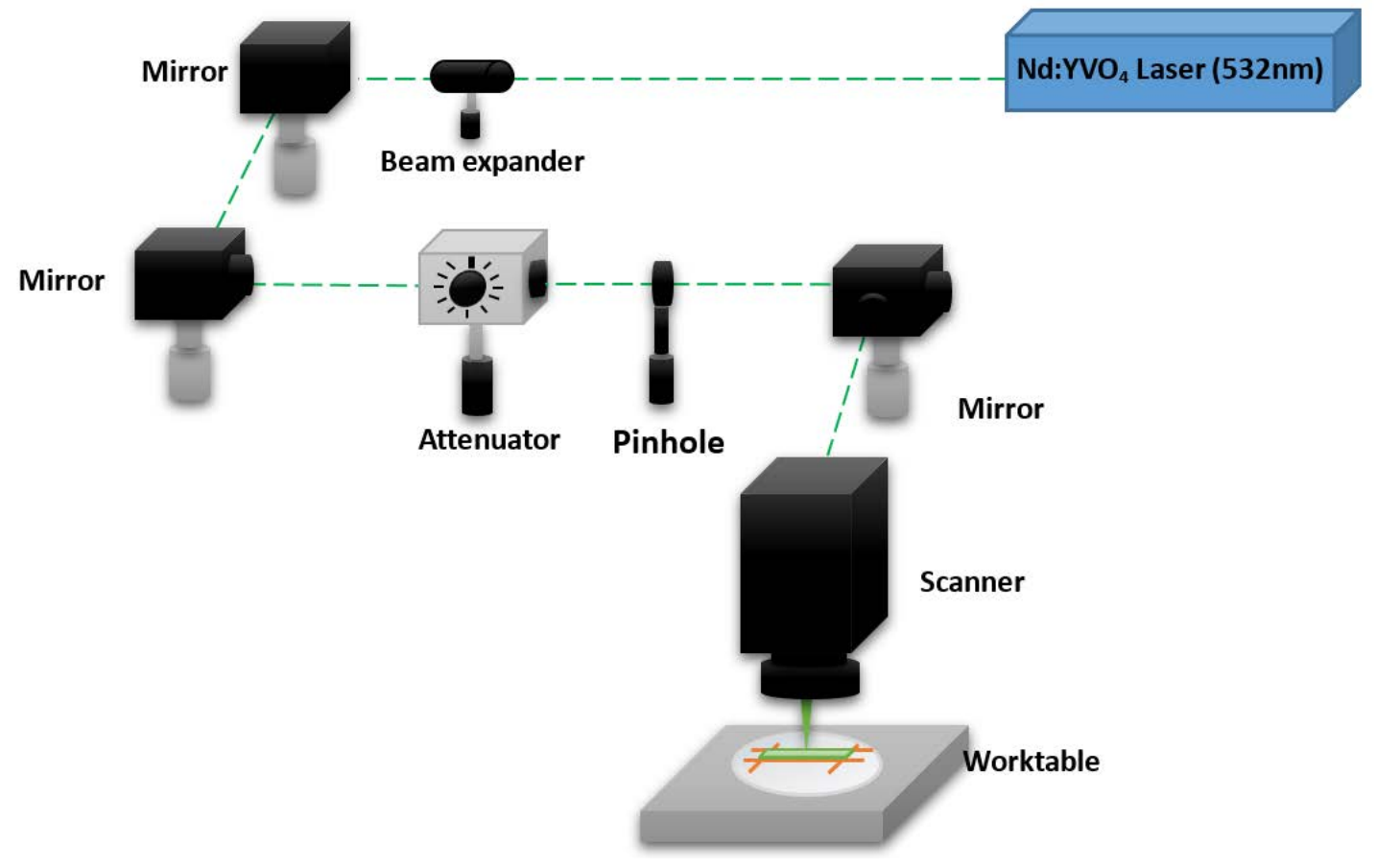

Figure 4.1: Schematic Explore set-up for the LIFT process. 


\subsection{2 $532 \mathrm{~nm}$ Picosecond laser set-up}

The picosecond laser source of $532 \mathrm{~nm}$ used in this thesis work is an EKSPLA uab laser (Atlantic 355-60, serial number DPL037), emitting in three different wavelengths: 1064 (IR), 532 (VIS), and 355 (UV) nm.

The laser source has been mounted onto a test bench which consists of a Granite base, a XY translation stage with working area of $500 \times 500 \mathrm{~mm}$ and speed up to $2 \mathrm{~m} / \mathrm{s}$, an optical system mounted onto an optical honeycomb breadboard, and an industrial PC for controlling the positioning system and the laser. The optical path comprises four high power mirrors and a fixed focusing lens (Linos Focus-Ronar) with focal length $58 \mathrm{~mm}$ and a Polygon scanner (NextScan LSE170). The polygon scanner can provide 100 to 400 line scans per second and speed from 25 to $100 \mathrm{~m} / \mathrm{s}$. The utilization of this kind of scanner is to test the capability of LIFT process in the very high speed from the industrial fabrication point of view. Table 4.2 shows the technical data of the Ekspla Atlantic laser performance. The path for IR/VIS included also a beam expander 5X (Sill Optics). Fig. 4.2 shows the schematic of the optical path for Ekspla Atlantic 355-60 laser set-up.

\begin{tabular}{|c|c|}
\hline \multicolumn{2}{|c|}{ General Characteristics } \\
\hline Wavelength & $532 \mathrm{~nm}$ \\
\hline Average Output power & $>35 \mathrm{~W} @ 400 \mathrm{kHz}$ \\
\hline Pulse Repetition rate range & $400-1000 \mathrm{kHz}$ \\
\hline Pulse duration (FWHM) & $<13 \mathrm{ps}$ \\
\hline Gain Medium & $\mathrm{Nd}: \mathrm{YVO}_{4}$ \\
\hline Spatial mode & $\mathrm{TEM}_{00}$ \\
\hline Spatial mode & $<1.3$ \\
\hline \hline Quality (M ${ }^{2}$ Beam Characteristics & $\mathrm{TEM}_{00}$ \\
\hline \hline
\end{tabular}

Table 4.2: Ekspla Atlantic laser performance specifications. 


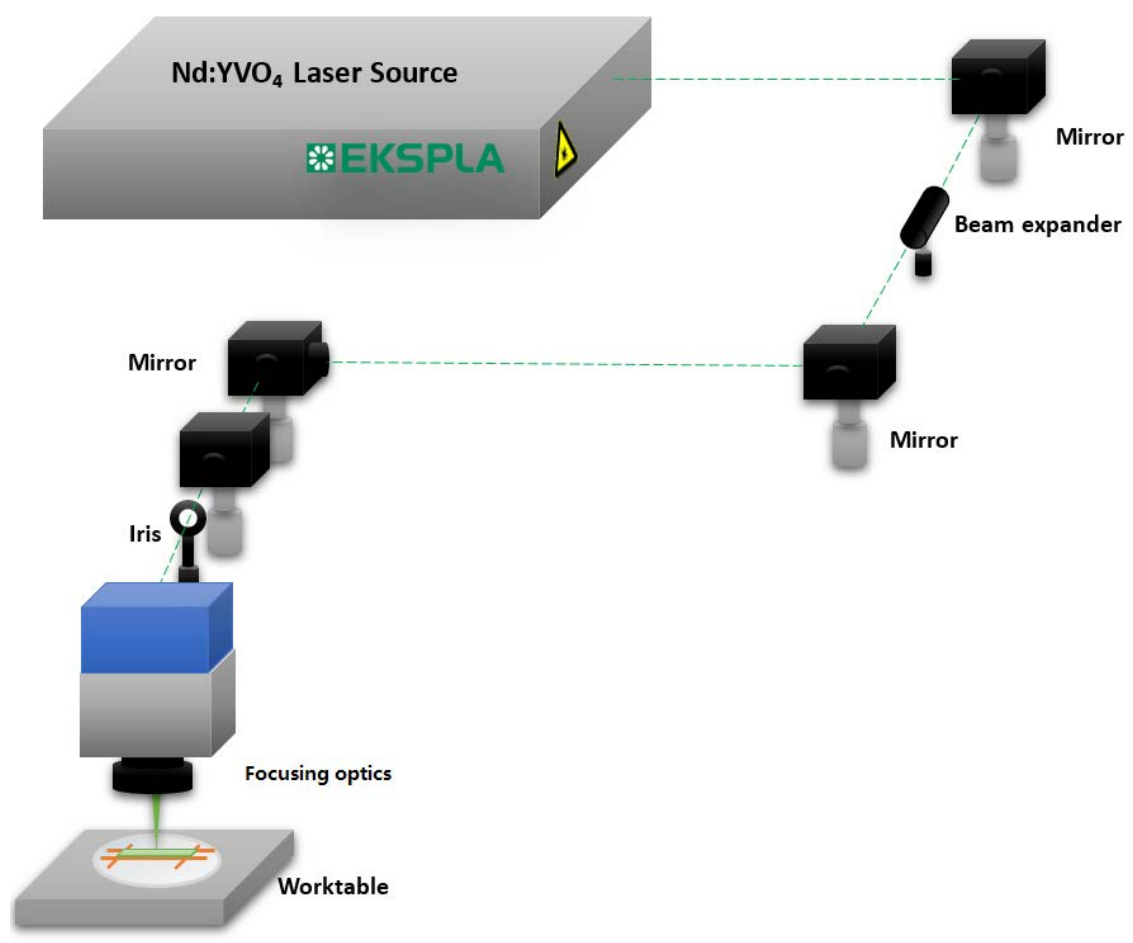

Figure 4.2: Schematic of optical path for Ekspla Atlantic 355-60 laser set-up.

\subsection{3 $1064 \mathrm{~nm}$ Picosecond laser set-up}

For the comparison of the effect on wavelength of LIFT experiment, a picosecond laser (Onefive, Katana-10 HP, serial number 826), emitting in $1064 \mathrm{~nm}$ wavelength is employed. Table 4.3 shows the standard specifications and the factory tested data of the laser, as shown in the equipment manual.

The laser source has been mounted onto the same test-bench. And the optical path comprises four high power mirrors, a beam expander 2X (Sill Optics), and a 2D laser beam deflection unit (Raylase SS-II-15) which allows high speed (up to $10 \mathrm{~m} / \mathrm{s}$ ) with marking area of $180 \times 180 \mathrm{~mm}$.

\begin{tabular}{|c|c|}
\hline \multicolumn{2}{|c|}{ General Characteristics } \\
\hline \hline Wavelength & $1064 \mathrm{~nm}$ \\
\hline Average Output power & $16 \mathrm{~W} @ 2000 \mathrm{kHz}$ \\
\hline Pulse Repetition rate range & $25-2000 \mathrm{kHz}$ \\
\hline
\end{tabular}




\begin{tabular}{|c|c|}
\hline Pulse duration & $35 \mathrm{ps}$ \\
\hline Gain Medium & $\mathrm{Nd}: \mathrm{YVO}_{4}$ \\
\hline Spatial mode & TEM $_{00}$ \\
\hline \multicolumn{2}{|c|}{ Beam Characteristics } \\
\hline Spatial mode & TEM $_{00}$ \\
\hline Quality $\left(\mathrm{M}^{2}\right)$ & $<1.3$ \\
\hline
\end{tabular}

Table 4.3: Onefive Katana-10 HP laser performance specifications for $1064 \mathrm{~nm}$ of wavelength.

\subsection{4 $532 \mathrm{~nm} \mathrm{CW}$ laser set-up}

For the purpose of functionalization of the deposited metal line by LIFT process, a CW green laser (Millennia Pro s-Series 15S J, Spectra-Physics) is applied for the laser curing/firing/sintering process. The laser system consists of a water-cooled laser head, a controller and a Model J80 air-cooled power supply.

\begin{tabular}{|c|c|}
\hline \multicolumn{2}{|c|}{ General Characteristics } \\
\hline \hline Wavelength & $532 \mathrm{~nm}$ \\
\hline Power & $0.2-15 \mathrm{~W}$ \\
\hline Gain Medium & $\mathrm{Nd}: \mathrm{YVO}_{4}$ \\
\hline \multicolumn{2}{|c|}{ Beam Characteristics } \\
\hline Spatial mode & TEM $_{00}$ \\
\hline \hline Quality (M ${ }^{2}$ ) & $<1.3$ \\
\hline
\end{tabular}

Table 4.4: Millennia Pro s laser output characteristics.

The laser source of Millennia and its optical path share the same positioning system with the Explore laser and the schematic of the CW laser system is illustrated in Fig. 4.3 


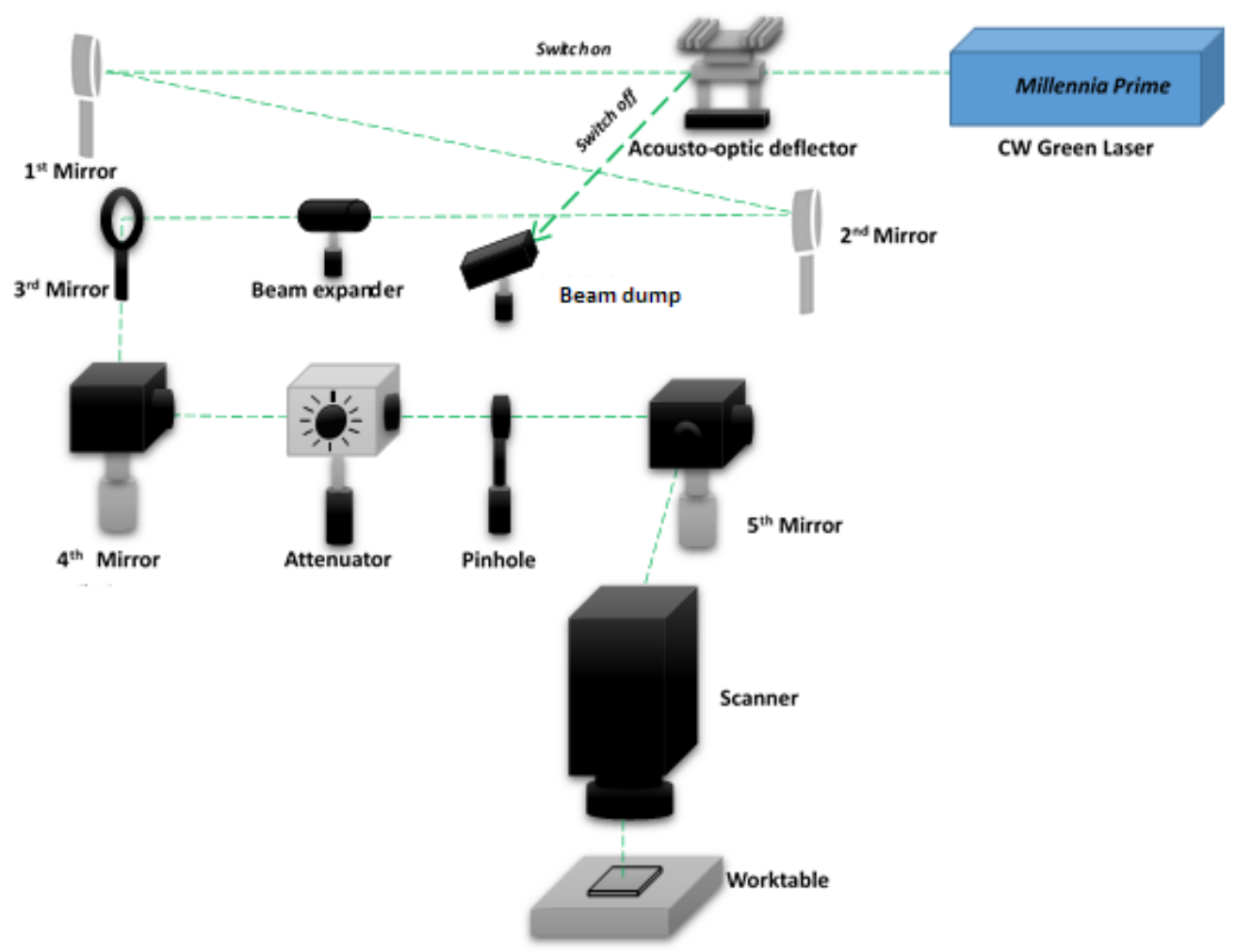

Figure 4.3: Schematic of CW 532nm laser system.

The positioning system consists of three parts: motor system, 3-axis worktable, controlling software. Instead of moving the scanner, it allows the worktable to move in three dimensions. The worktable can be moved precisely inside the tracks which are connect to the controller in the $\mathrm{X}, \mathrm{Y}$ plane and $\mathrm{Z}$ axis for positioning the sample. In these experiments, the sample should be put under the focus position of the laser spot. The motor system consists of the Parker 6k4 4-axis servo/stepper controller, MW power supply and Aries servo drives. It can be programed with the Motion Planner software to setup the object-oriented code and routine task. In this thesis work, only the $z$ axis is employed.

The four laser set-ups are listed in Table 4.5. 


\begin{tabular}{|c|c|c|c|c|c|c|}
\hline $\begin{array}{c}\text { Experimental set- } \\
\text { up }\end{array}$ & $\begin{array}{l}\text { Spectra- } \\
\text { Physics } \\
\text { Explore }\end{array}$ & \multicolumn{2}{|c|}{$\begin{array}{l}\text { Ekspla } \\
\text { Atlantic }\end{array}$} & \multicolumn{2}{|c|}{$\begin{array}{l}\text { Onefive } \\
\text { Katana }\end{array}$} & $\begin{array}{l}\text { Spectra- } \\
\text { Physics } \\
\text { Millennia }\end{array}$ \\
\hline Focusing optics & $\begin{array}{c}\text { Galvo } \\
\text { scanner } \\
\text { (Scanlab } \\
\text { HurrySCAN } \\
\text { II) }\end{array}$ & $\begin{array}{l}\text { Fix lens } \\
\text { (Linos } \\
\text { Focus- } \\
\text { Ronar) }\end{array}$ & $\begin{array}{l}\text { Polygon } \\
\text { scanner } \\
\text { (NextScan } \\
\text { LSE170) }\end{array}$ & $\begin{array}{l}\text { Fix lens } \\
\text { (Linos } \\
\text { Focus- } \\
\text { Ronar) }\end{array}$ & $\begin{array}{c}\text { Galvo } \\
\text { scanner } \\
\text { (Raylase } \\
\text { SS-II-15, } \\
\text { Linos F- } \\
\text { Theta- } \\
\text { Ronar) }\end{array}$ & $\begin{array}{c}\text { Galvo } \\
\text { scanner } \\
\text { (Scanlab } \\
\text { HurrySCAN } \\
\text { II) }\end{array}$ \\
\hline $\begin{array}{l}\text { Repetition rate } \\
\qquad(\mathrm{kHz})\end{array}$ & 20 & 396 & 598 & 30 & 30 & - \\
\hline $\begin{array}{c}\text { Maximum Power } \\
\text { (W) }\end{array}$ & $0.7 \pm 0.01$ & $22.7 \pm 0.1$ & $11.5 \pm 0.1$ & $0.33 \pm 0.1$ & $0.68 \pm 0.01$ & $7.5 \pm 0.1$ \\
\hline $\begin{array}{l}\text { Wavelength } \\
\text { (nm) }\end{array}$ & 532 & & 32 & & & 532 \\
\hline $\begin{array}{c}\text { Pulse } \\
\text { duration(FWHM) }\end{array}$ & $\begin{array}{c}<15 \mathrm{~ns} \text { at } \\
50 \mathrm{kHz}\end{array}$ & & 3 ps & & ps & - \\
\hline $\begin{array}{c}\omega_{0}(\mu \mathrm{m}) \text { (by Liu } \\
\text { Method) }\end{array}$ & $12.5 \pm 1$ & $4 \pm 1$ & $14 \pm 1$ & $8 \pm 1$ & - & $17.5 \pm 1$ \\
\hline
\end{tabular}

Table 4.5: Experimental set-up used in the thesis.

\subsection{Beam waist Measurement}

For measuring the laser beam waist after focusing lens. Two methods have been employed. The Liu's method [87] has been used for rapid determination of the spot size. 
This method is also widely applied as an experimental way to calculate the damage threshold.

For a general Gaussian laser beam, the spatial and temporal beam profile is given by the follow Equation

$$
I(r, t)=I_{0} e^{-\frac{r^{2}}{\omega_{0}^{2}}} e^{-\frac{t^{2}}{\tau^{2}}}
$$

where $I_{0}$ is the laser peak intensity, $\omega_{0}$ is the spatial radius and $\tau$ is the temporal pulse width at $1 / \mathrm{e}^{2}$ intensity contour, $r$ is the radial coordinate distance from the propagation axis.

And the spatial distribution of the energy fluence is given by integration of Equation (4.4),

$$
\Phi(r)=\int_{-\infty}^{\infty} I(r, t) d t=\Phi_{0} e^{\frac{-2 r^{2}}{\omega_{0}^{2}}}
$$

where $\Phi_{0}=\sqrt{\pi} \tau I_{0}$ is the peak fluence at the beam center. $\Phi_{t h}$ is define by damage threshold fluence for the material. Then the diameter as a function of the fluence is obtained by Equation

$$
D^{2}=2 \omega_{0}^{2} \ln \left(\frac{\Phi_{0}}{\Phi_{t h}}\right)
$$

By substituting the peak fluence as a function of the pulse energy, $\Phi_{0}=\frac{2 E_{p}}{\pi \omega_{0}^{2}}, \Phi_{t h}=$ $\frac{2 E_{t h}}{\pi \omega_{0}^{2}}$, Equation (4.5) is given by 


$$
D^{2}=2 \omega_{0}^{2} \ln \left(\frac{E_{p}}{E_{t h}}\right)=2 \omega_{0}^{2} \ln \left(\frac{2 E_{p}}{\pi \omega_{0} \Phi_{t h}}\right)
$$

Hence, the diameter of the crater is measured and plotted as a linear function of $\ln E_{p}$, as Fig. 4.4 shows, and the slope of the plot $\varphi$ is then $2 \omega_{0}^{2}$. Then the beam waist is given by

$$
\omega_{0}=\sqrt{\frac{\varphi}{2}}
$$

And the propagation error of $\Delta \omega_{0}$ is given by

$$
\Delta \omega_{0}=\frac{\Delta \varphi}{2 \sqrt{2 \varphi}}
$$

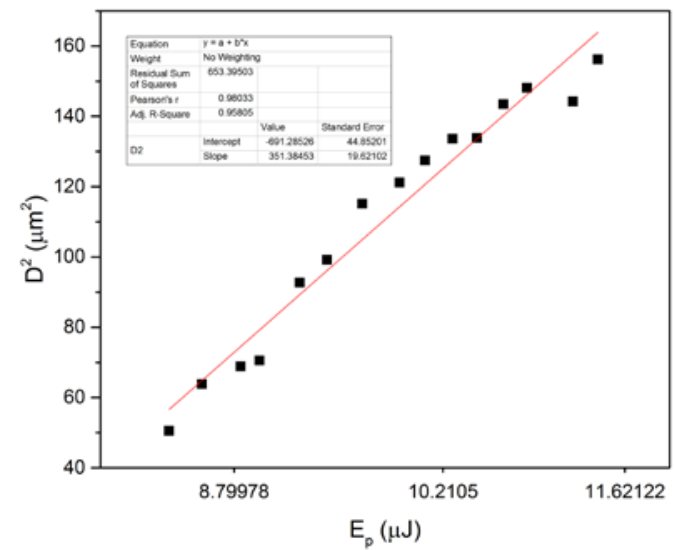

Figure 4.4: Crater diameter represented as a function of laser pulse energy for $20 \mathrm{kHz}$ of repetition rate with the corresponding fitting line. 
The other method for the beam waist determination is to use a beam profiler. (Spiricon SP620U). Images of a strongly attenuated laser beam have been recorded at different distances $(z)$ to the focal position
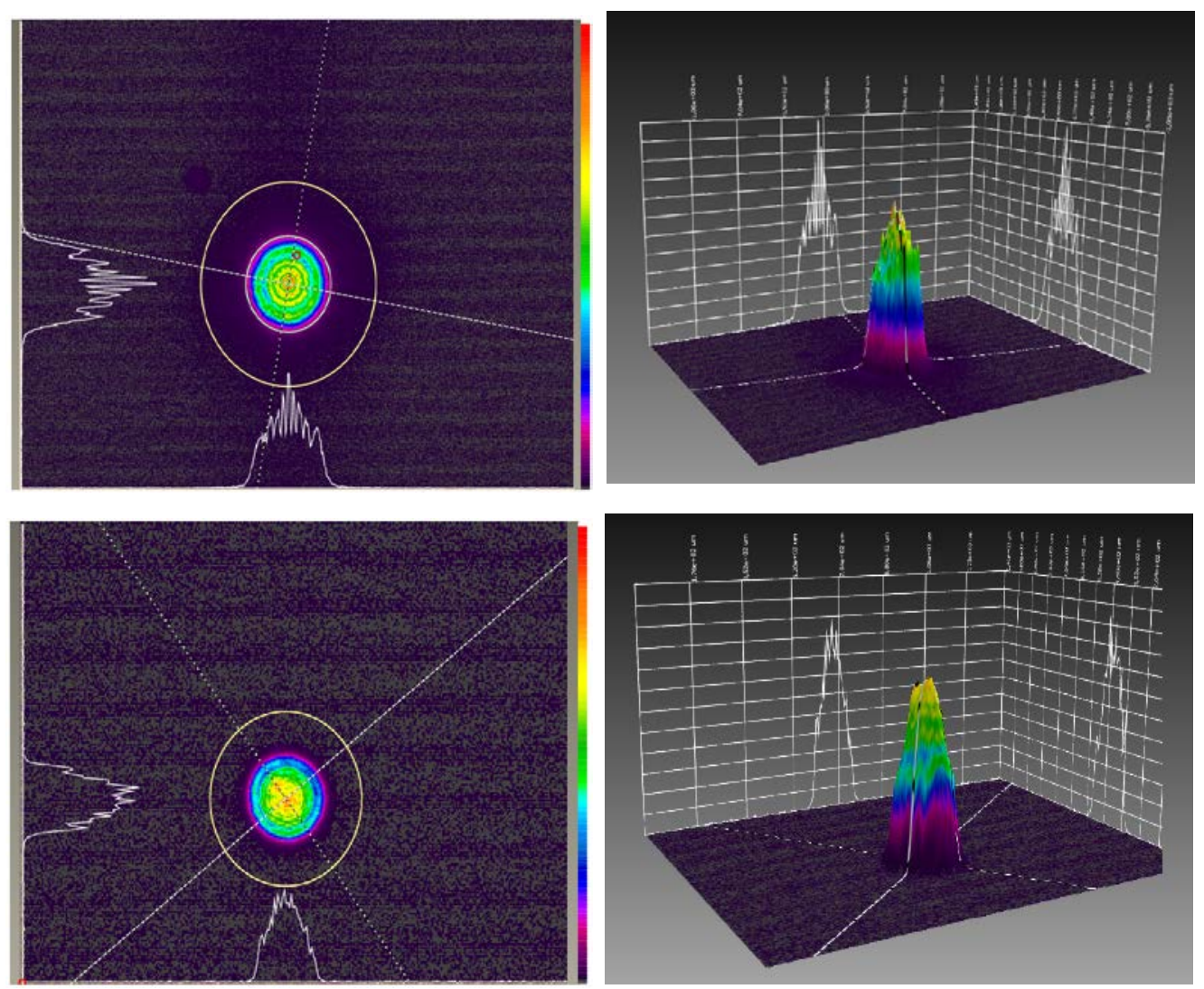

Figure 4.5: (Left) 2D and (right) 3D profile of (top) the IR beam in a pre-focus position, (bottom) VIS beam in a post-focus position.

Beam diameters at different $z$ positions have been measured using the software included with the camera (Spiricon BeamMic), which integrates the energy distribution (see Fig. 4.5) and calculates the beam diameter. An example of beam diameter of Ekspla laser measured with the beam profiler for the optical scanner are shown in Fig. 4.6. $\omega_{0}$ has been determined as the smallest beam waist by fitting of the beam diameter data to a hyperbola. 


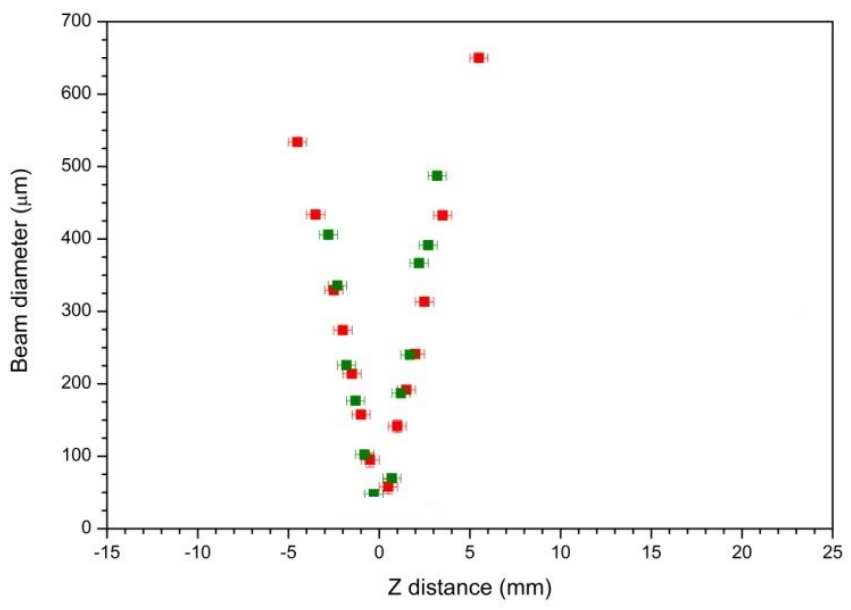

Figure 4.6: Beam diameter measured with the beam profiler for the optical scanner.

\subsection{Donor preparation}

The donor substrate used in the LIFT experiment consists of a laser-transparent carrier, which is the glass slide, and the silver paste as transfer material.

\subsubsection{Silver paste preparation}

Silver pastes with high conductivity, designed for rapid dry and spike firing, are commonly used for the standard screen printing process in the photovoltaic metallization [88-91]. A good silver paste should provide the final printed contacts featured with high line conductivity, low contact resistance to the underlying emitter, a high aspect ratio cross sectional profile and a good mechanical adhesion to the cell substrate surface. The systematic combination of multiple inorganic and organic components with uniform dispersion in the paste distinguish the features of one from another. Nevertheless, the fundamentally functional constituents are relatively similar [90]. The organic solvent serves as the temporary carrier, which will be evaporated and decomposed during the drying/firing process and leave only inorganic components like silver powder and glass frit on the wafer. Combined with binding agents the organic solvents prevent silver 
particles from interaction and affects the rheological behavior to obtain the optimum printing quality.

As the highest proportion of solid fraction of silver paste, silver powder provides conductivity in the front metal contact. And the glass frit helps to etch the antireflection layer to form good ohmic contact and serves as well as binder to enhance the adherence with substrate in the crystalline silicon solar cell [92]. The glass frit can also influence the contact formation process, the melting point of the silver and conductivity of the finger.

A commercial silver paste (DuPont Solamet PV17F) was used as the donor material for LIFT. It is a highly conductive silver paste that provides excellent efficiency, reliable soldered adhesion, low lay down, rapid dry, and very fast firing. Although it is designed for screen-printing, its excellent electrical and curing/sintering properties make it a good candidate for LIFT metallization. Table 4.6 shows the physical and chemical properties of the paste provided by the manufacturer. Unlike the thin liquid films such as inks or with other Newtonian fluid, silver paste has high viscosity and its different rheology can be observed in the LIFT process. Nevertheless, such behavior results in an increased height in the printed lines, which is required for extending the cross-section area of conductive traces with low line resistance in the contact fingers.

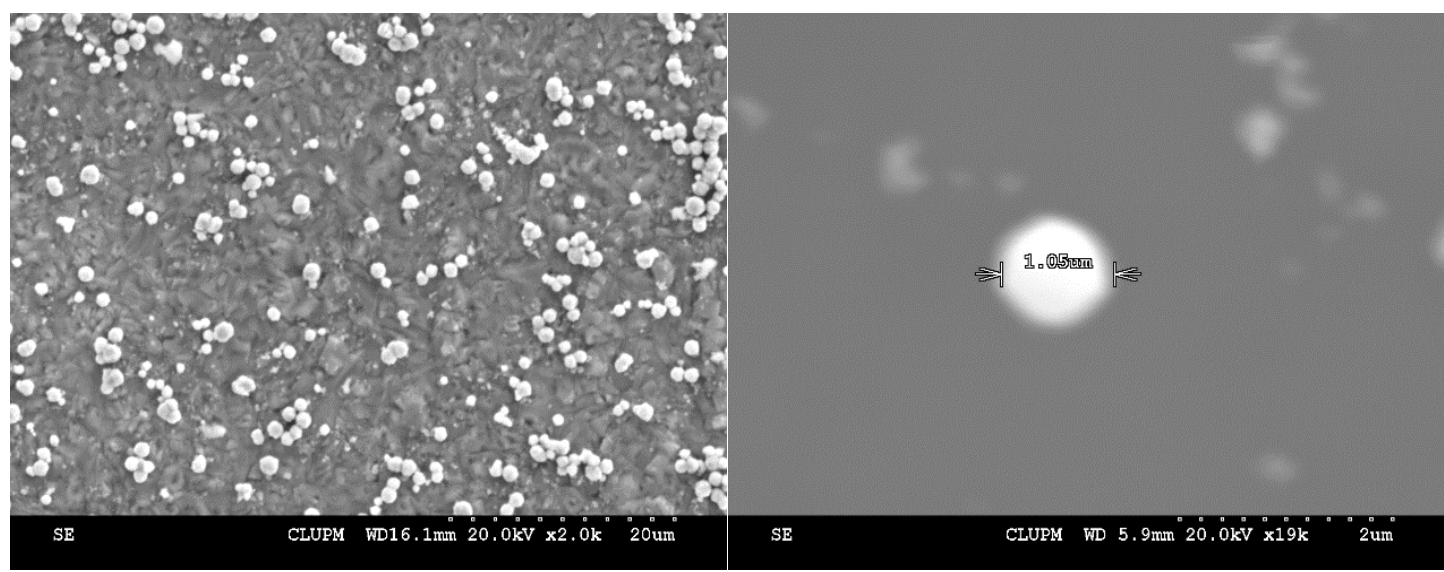

Figure 4.7: Micro-sized silver particles distributed on the CIGS substrate surface 


\begin{tabular}{|c|c|}
\hline $\begin{array}{l}\text { Viscosity } \\
\text { (Brookfield HBT, } 10 \text { rpm SC4-14/6R } \\
\text { utility cap and spindle, 25ㅇ) }\end{array}$ & $280-400 \mathrm{~Pa} \cdot \mathrm{s}$ \\
\hline Solid Content at $750 \stackrel{\circ}{C}$ & $89.5-91.0 \%$ \\
\hline Resistivity & $<5 \mathrm{~m} \Omega / \mathrm{sq} / \mu \mathrm{m}$ \\
\hline Silver grain & $1-4 \mu \mathrm{m}$ \\
\hline Organic carrier & $\begin{array}{l}\text { N,N'-Ethane-1,2-diylbis(decanamide) } \\
\text { 12-Hydroxy-N-[2-[1- } \\
\text { oxydecyl)amino] ethyl]octadecanamide } \\
\text { N,N'-Ethane-1,2-diylbis(12- } \\
\text { hydroxyoctadecanamide) }\end{array}$ \\
\hline Thinner & 9450 \\
\hline
\end{tabular}

Table 4.6: DuPont Solamet ${ }^{\circledR}$ PV17F properties [93]

The silver particles of PV17F paste have a size of 1-4 $\mu \mathrm{m}$ with fine spherical form for achieving a large contact area and high packing density. It can be also observed several agglomerations of the silver particles, which is usually regarded as an issue for the screen printing because it can produce clogging due to the screen opening size. However, in the LIFT process the high-viscosity silver paste voxel is freeform-released by the laser pulsed induced pressure filed without any spatial limitation.

In order to make the LIFT line functionalized, the subsequent treatment of sintering or firing need to be applied. In the thesis work, in order to have a comparison, a furnace is used for the laser curing/sintering process. The printed line is commonly treated in a firing furnace Due to the data sheet of the Solamet ${ }^{\circledR}$ PV17F silver paste, to get the best electrical contact performance the fast firing process can be applied in the furnace with the thermal budget above $600^{\circ} \mathrm{C}$ should be kept to minimum, ideally smaller than 8 seconds and the silver paste should be fired at a peak temperature around $800^{\circ} \mathrm{C}$ [93]. 
Whereas, in the other case with the CIGS substrate, since the top layer is a TCO film which is a conductive layer, there is no need for the fast firing process for the entire cell structure. In addition, the CIGS substrate can sustain a maximum temperature of $200^{\circ} \mathrm{C}$, hence if applied a furnace treatment, the LIFT lines formed on the substrate can be only treated with the curing process. After the heat treatment, the wafer can be directly cooled down to room temperature or kept at an elevated temperature for a short period as an annealing process for improve the finger conductivity. In this case, the CIGS substrate with the deposited LIFT lines were put in the furnace at $200^{\circ} \mathrm{C}$ for 1 hour. And then directly cooled down to room temperature.

According to PV17F paste datasheet, it has a viscosity between 280 and $400 \mathrm{~Pa} \cdot \mathrm{s}$. In order to investigate the silver paste fluid behavior and its influence for the LIFT process, the viscosity value of the paste needs to be measured. Due to the small sample volume $(5 \mathrm{~mL})$, the rheological analysis was accomplished by a cone spindle \& plate viscometer (Wells/Brookfiel Viscometer HBDV-II; Cone spindle CPE-52). The viscometer shear rate was set at 2, 5, 10, 20, $40 \mathrm{~s}^{-1}$ and the viscosity readings were recorded in $60 \mathrm{~min}$. The silver paste exhibits non-Newtonian thixotropic fluid with pseudoplastic (viscosity decreases with increasing shear rate until an equilibrium value is reached) and thixotropic (viscosity at a constant shear rate decreases with eventually steady out to an equilibrium value) behavior (see Fig. 4.8).

Due to the large influence of the viscosity on the quality of the printing line resolution $[94,95]$, the exact viscosity value of the paste was measured before every experiment. If aging, due to organic evaporation, is detected (showing a higher viscosity) then some thinner must be added to obtain the viscosity of reference, which is $250 \pm 30 \mathrm{~Pa} \cdot \mathrm{s}$ [93].

Hence, a study of viscosity behavior with modification of wt\% of thinner was performed in order to adjust the viscosity of paste. The thinner addition is varied by its weight percentage from $2,4,5,6,8$ to $10 \%$ of $15 \mathrm{~g}$ silver paste using a fixed shear rate of $5 \mathrm{~s}^{-1}$. 

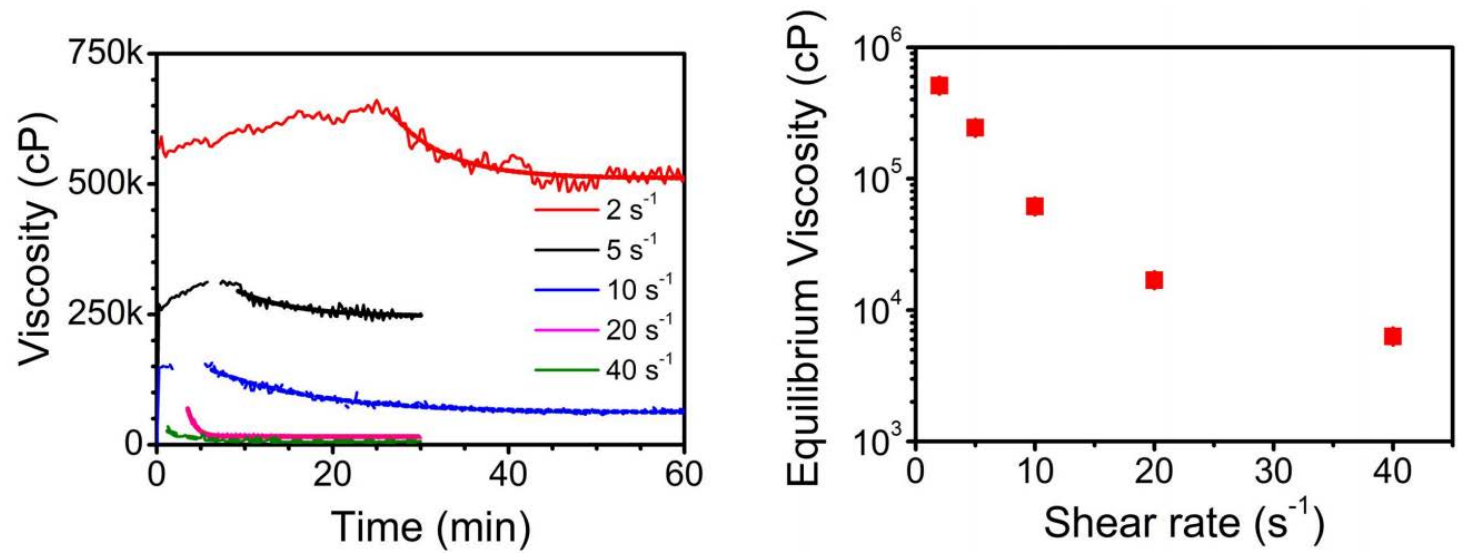

Figure 4.8: Silver paste viscosity showing the pseudo-plastic and thixotropic behavior. (Left) Viscosity vs time at different shear rate (Right) Equilibrium viscosity vs shear rate [13].

Fig. 4.9 shows the viscosity curve with thinner addition of different weight percentage. And with more wt\% of thinner adding to the silver paste, the viscoelastic response period reduces, saying plateau value is obtained takes place more fast. By plotting the plateau viscosity value of the samples, it is observed clearly that viscosity decreases with increasing weight percentage of thinner.
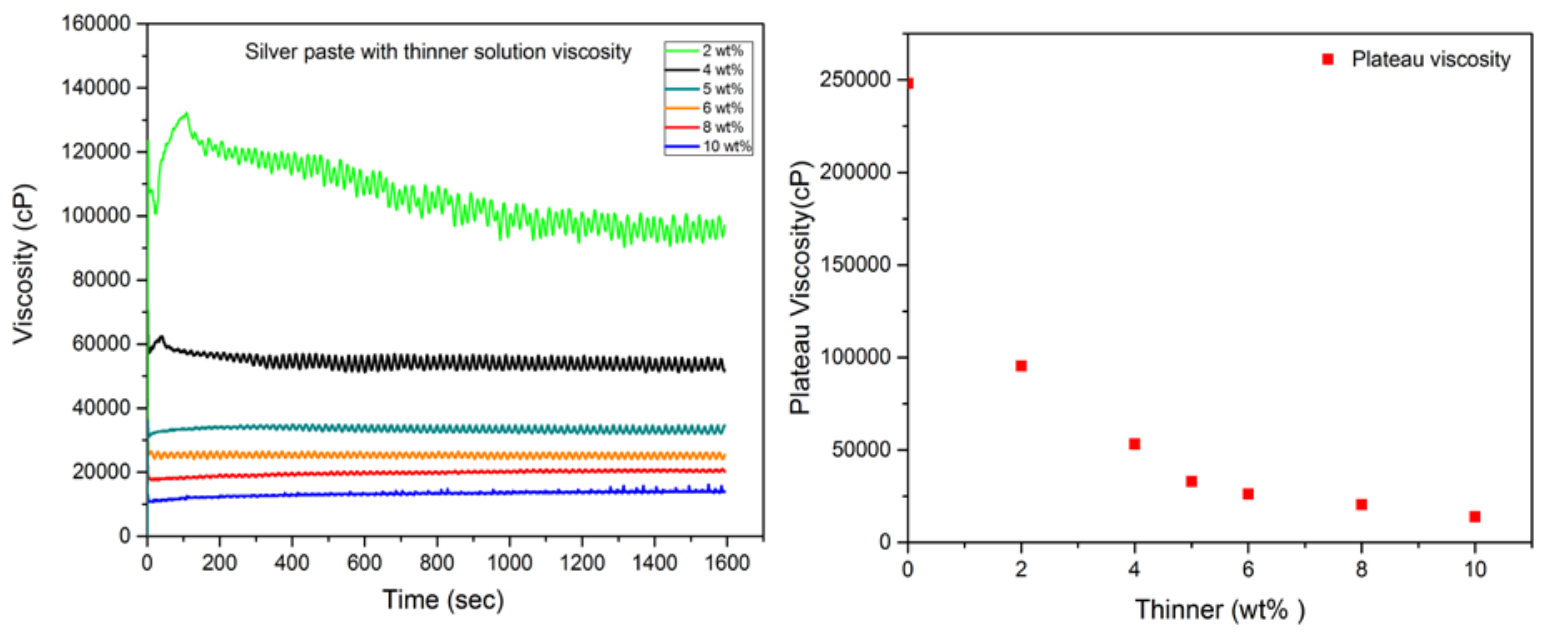

Figure 4.9: Viscosity of silver paste with varying weight percentage of thinner (Left). Plateau viscosity of silver paste as a function of wt\% of thinner. 


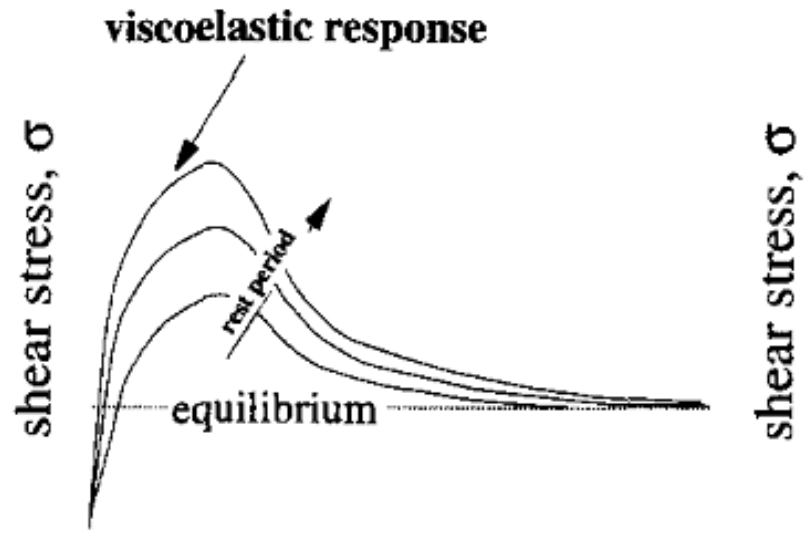

shearing time, $\mathrm{t}$

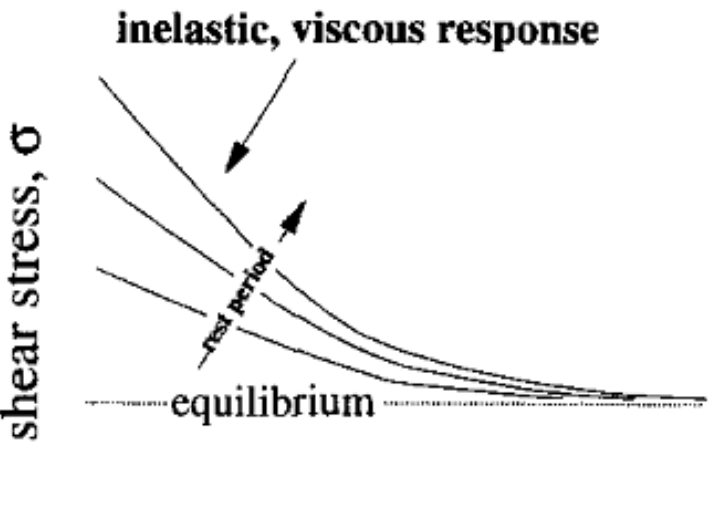

shearing time, $\mathrm{t}$

Figure 4.10: Different kinds of thixotropic behavior on startup of shear after various degrees of rest [96].

The silver paste needs to be measured before every experiment and before using it one should gently stirred it for 5 minutes to attain the equilibrium viscosity due to its thixotropic behavior. As Fig. 4.10 illustrates, it is practically important that with longer rest period, the recovered viscosity will be initially higher. Hence, after the silver paste preparation, it is better to initialize the LIFT experiments as soon as possible within the period of viscous response of the paste.

The silver paste is then coated onto glass slides by using a commercial blade coater (Control Coater model 101, RK PrintCoat Instruments Ltd). Firstly, the glass slide is fixed on the coating bed by Kapton adhesive tapes with a defined thickness for leaving the margins on the edges of the glass slide. Then by adjusting two micrometer thumbscrews of the film applicator head, the gap distance between the blade and the glass slide surface can be controlled in $10 \mu \mathrm{m}$ increments. The thickness was measured in each donor sample before and after LIFT experiments using confocal microscopy. 


\subsubsection{Gap set-up}

In the LIFT experiment, "Gap" is referred to the distance between the acceptor substrate surface plane and the donor film (silver paste) surface plane. As the first beginning, the gap size is set by the Kapton $\mathrm{HN}^{\circledR}$ polyimide films with defined thickness. By sticking two film strips at the silver paste surface and overlapping the others two strips on top of the previous ones, the gap size can be varied by the combinations of different Kapton film thickness (see Fig. 4.11).
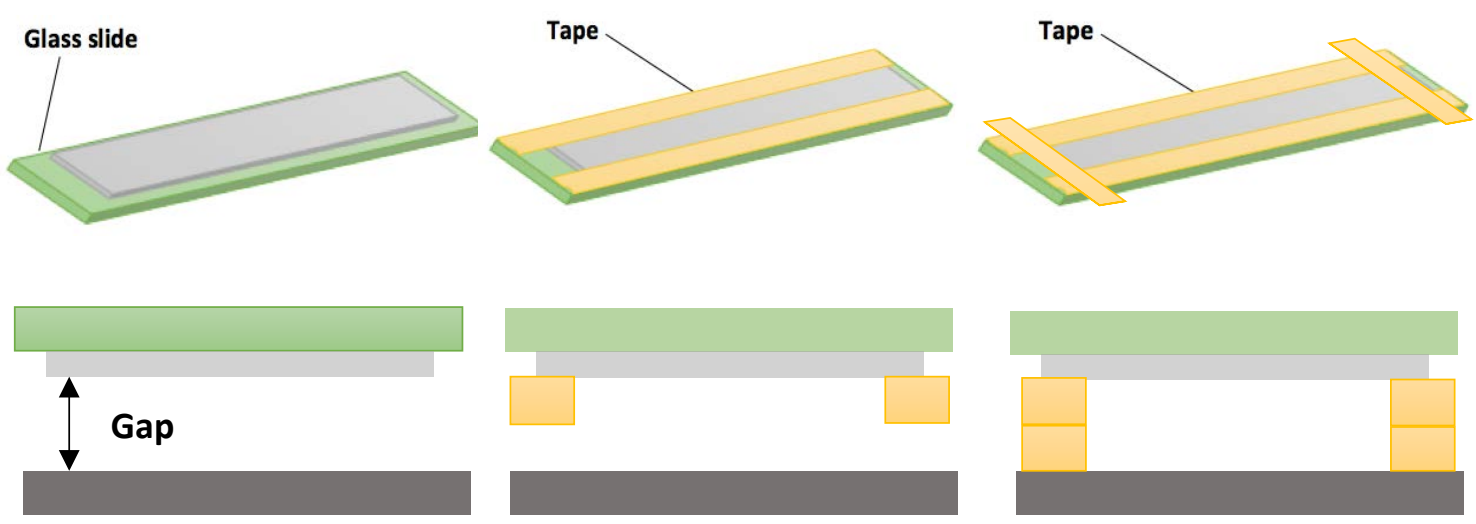

Figure 4.11: Gap size set by Kapton ${ }^{\circledR} \mathrm{HN}$ polyimide films used in the LIFT experiment.

Three Kapton films with thickness of 13, 25 and $50 \mu \mathrm{m}$ were used as the gap set-up. Hence, 6 different gap size $d_{g a p}$ are obtained by the sum of two film thickness, see Table 4.7.

$$
d_{g a p}=t_{1}+t_{2}
$$

\begin{tabular}{|c|c|c|c|}
\hline & $\mathbf{1 3}$ & $\mathbf{2 5}$ & $\mathbf{5 0}$ \\
\hline $\mathbf{t}_{\mathbf{2}}$ & $\mathbf{t}_{\mathbf{1}}$ & & \\
\hline $\mathbf{1 3}$ & 26 & $*$ & $*$ \\
\hline $\mathbf{2 5}$ & 38 & 50 & $*$ \\
\hline $\mathbf{5 0}$ & 63 & 75 & 100 \\
\hline
\end{tabular}

Table 4.7: The combination of three Kapton ${ }^{\circledR}$ film thicknesses; * Repeated combination. 
Gap setting by Kapton films is an easy, rapid and efficient way for most of the LIFT experiments. However, it is also a rough method for define the gap size which and has a high uncertainty since it is reduced by the deformation in the edges of silver paste caused by the donor's own weight (see Fig. 4.12).

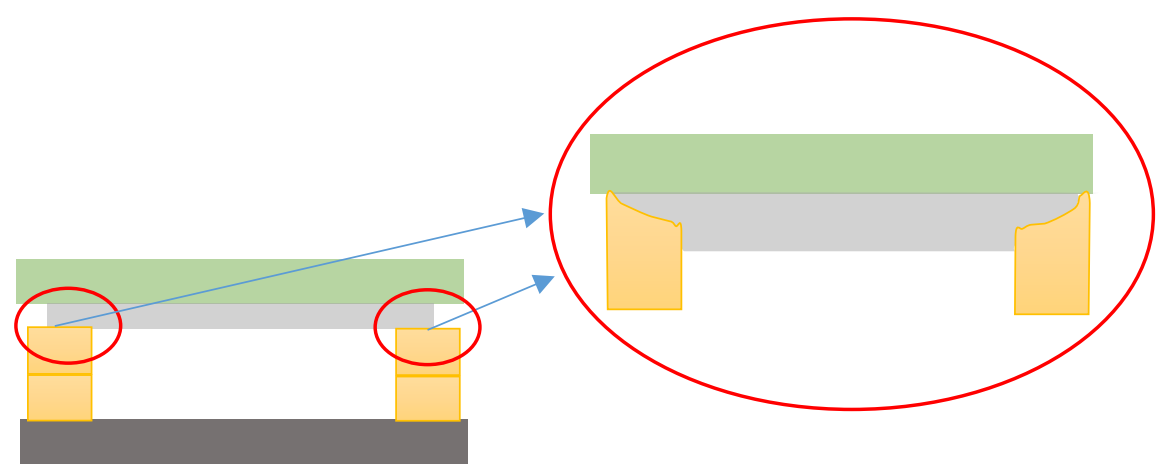

Figure 4.12: Deformation in the edges of the silver paste caused by the donor's own weight.

An improved method can be implemented by using two Kapton adhesive tapes stuck on the margin of the glass side (see Fig. 4.13). With this method, the gap size $d$ is expressed as

$$
d_{\text {gap }}=t_{\text {tape }}-t_{\text {paste }}
$$

where $t_{\text {tape }}$ is the total thickness of the Kapton tape and $t_{\text {paste }}$ is the thickness of the silver paste.

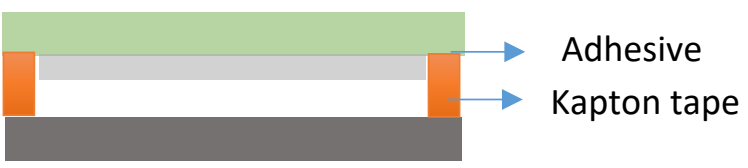

Figure 4.13: Gap set-up with Kapton ${ }^{\circledR}$ adhesive tape for silver paste donor. 
This method avoids the deformation caused by the polyimide film contact with the silver paste which is a non-Newtonian fluid. However, the gap size is constrained to some fixed values which are the total thickness of the Kapton tapes.

\subsection{Morphology characterization}

\subsubsection{Confocal Microscopy}

In the thesis work, the counter 2D images, topographical 3D images and profiles of the metal lines deposited by LIFT metallization process and cured lines have been characterized by a Confocal Microscope (Leica DCM 3D). Confocal microscopy provides a direct, non-contacting and non-destructive method for obtaining topographical images and surface profile of the sample.

By scanning a focused laser beam through a light source aperture and an objective lens into a sample, the detector can collect the reflective light, which is coming from the focal point, and obstruct the out-of-focus ones by the pinhole. Moving the focal plane at various depths with small steps in the $z$-direction, the $z$ stacks images can be processed by the computer software and provide information of the 3D reconstruction of the interior of the sample nondestructively.

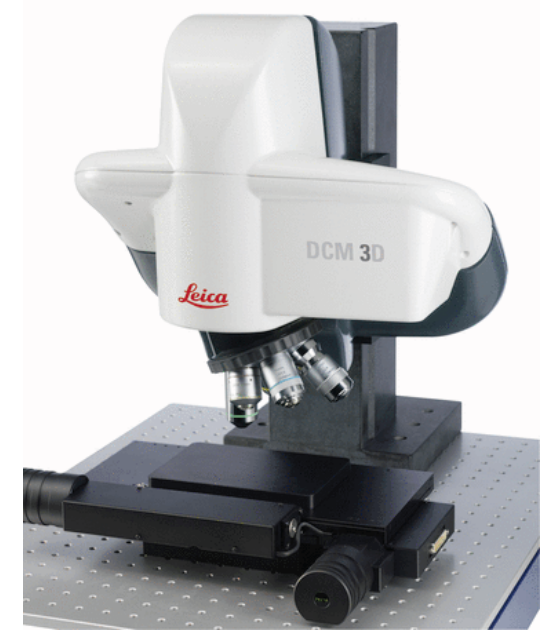

Figure 4.14: The Leica DCM3D Confocal Microscope used in the thesis. 
In the thesis work, the Leica DCM3D Confocal Microscope (see Fig. 4.14) has been used to study the morphology of the laser induced forward transferred voxel, the profile of the voxel per pulse or the cross-sectional profile data can be plotted for obtaining the information of the width, height, aspect ratio, cross section area etc.

\subsubsection{Scanning Electron Microscope (SEM)}

For the observation of the microstructures of the deposited voxel or lines by LIFT, a Hitachi S-300N SEM is employed (see Fig. 4.15). The system allows users to analyze sample at voltages between 1-30 kV and pressures from $<1 \mathrm{~Pa}$ to $270 \mathrm{~Pa}$. In this thesis work, SEM images gives a good view of the microstructure change when sintering takes place. It also plays an important role in the study of the wetting phenomena of the silver paste.

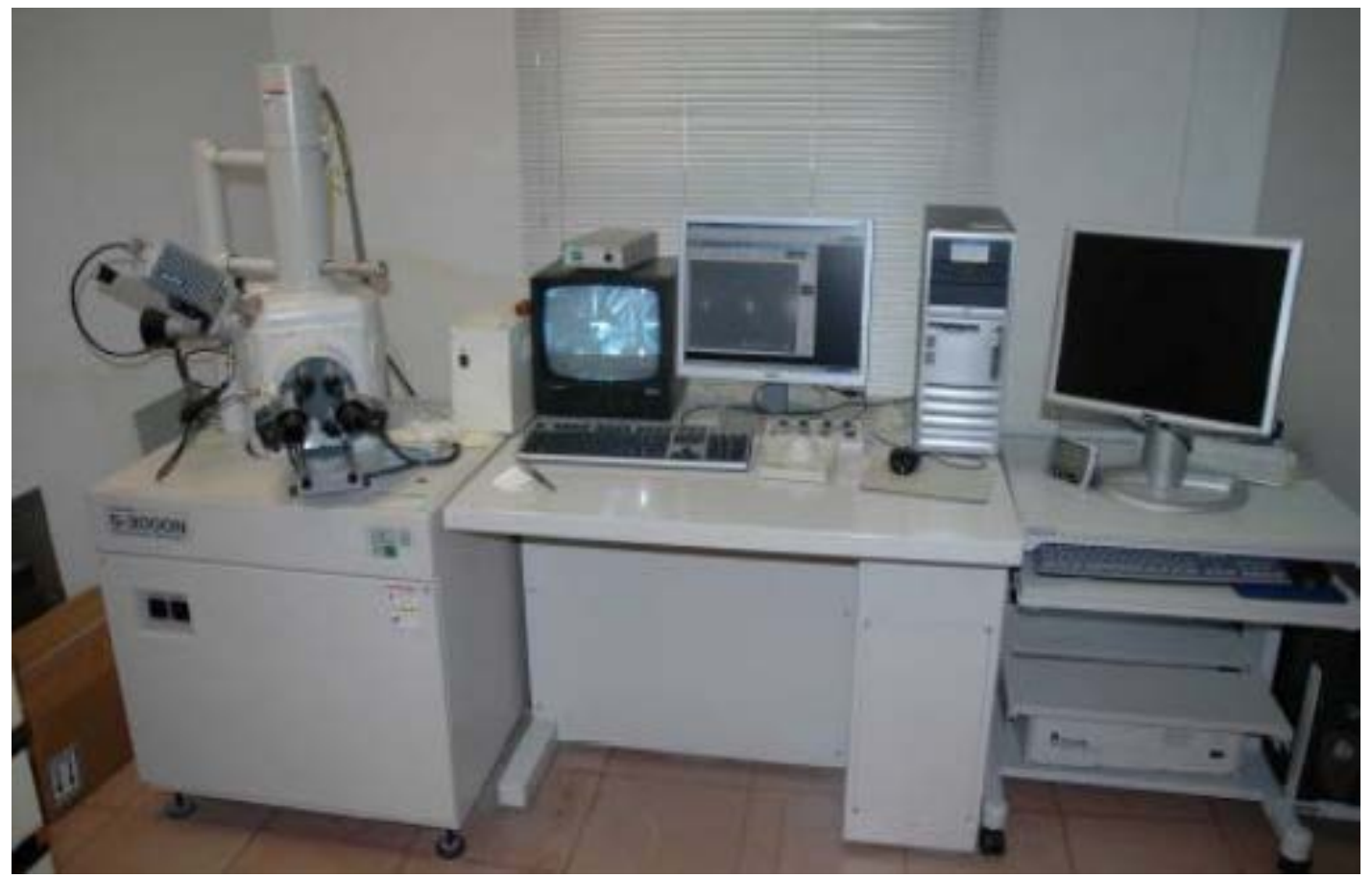

Figure 4.15: The SEM Hitachi S-3000N work station 


\subsection{Time-resolved imaging system}

In order to reveal the nature of the LIFT mechanism and explain the hydrodynamic behavior of the transfer process, a time-resolved imaging system is employed in cooperating with Professor Arnold from Princeton University [81].

The high-speed images system usually consists of several elements. A frequency-tripled $\mathrm{Nd}: \mathrm{YVO}_{4}$ laser (Coherent AVIA) emitting at $355 \mathrm{~nm}$ with a pulse duration of $20 \mathrm{~ns}$, was employed for initiating the LIFT process. The optical path consists of two dielectric mirrors and a neutral-density filter and a $10 \times$ laser objective (NA=0.25). The beam has approximately Gaussian shape. And the laser spot size is about $20 \mu \mathrm{m}$ which refers to the $1 / \mathrm{e}^{2}$ diameter of the beam.

The illuminating source is a 25 -ns plasma-discharge flash lamp (HSPS Nanolite) which sets the time resolution of the process and is strobed after a specified time delay relative to the laser pulse by a digital delay generator (SRS DG535). A magnification system composed of a microscope objective (InfiniTube with a Mitutoyo 50x or 10x Long-WD Objective) attached to a detector CCD camera. The scheme of the imaging system is illustrated in Fig. 4.16. The time-resolved images of LIFT from a $10 \mu \mathrm{m}$ film of $\mathrm{N}$-methyl2-pyrrolidone (NMP) is shown in Fig. 4.17 [97].

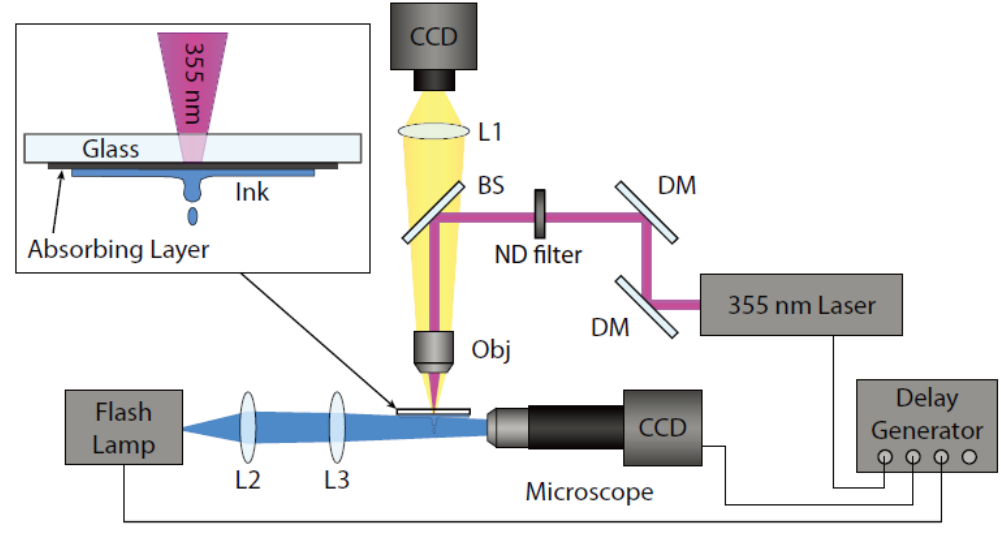

Figure 4.16: Diagram of the high-speed time-resolved imaging system [81]. 


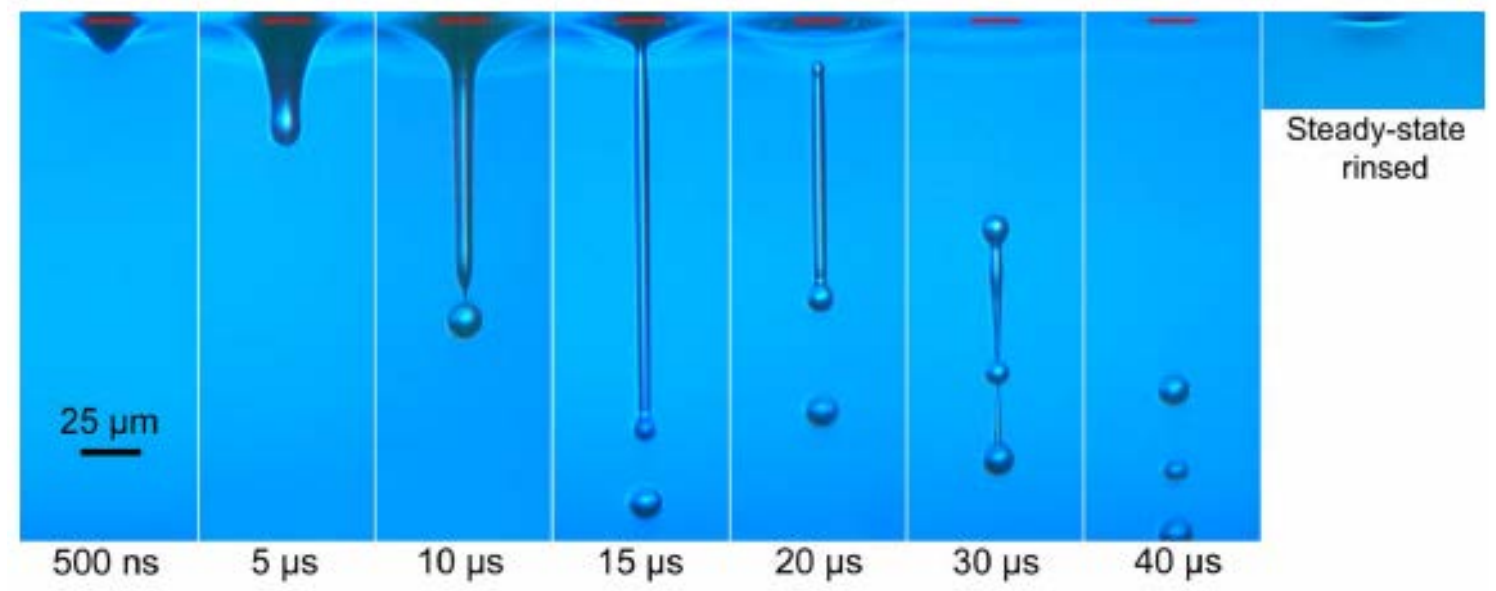

Figure 4.17: Time-resolved images of transfers from a $10 \mu \mathrm{m}$ film of $\mathrm{N}$-methyl-2pyrrolidone (NMP) by LIFT process [97].

\subsection{Electrical characterization method}

The electrical properties of LIFT deposited silver contacts are essential for optimizing the solar cell performance. As previously demonstrated in Chapter 2, both bulk resistivity and specific contact resistance play important roles in the serial resistance. Hence, for design and optimize the grid and bus structure, it's useful to quantify the bulk resistivity of the deposited silver line and the specific contact resistance between the silver line and the substrate.

\subsubsection{Finger bulk resistivity measurement}

The resistivity of the silver paste line depends upon several factors, including the material structure, doping, processing, and environmental factors such as temperature and humidity. To study the characteristics of the LIFT processed metal contacts, a multimeter is used in the tests. However, the four-point probe technique is a better method of measuring thin, flat materials. It involves four probes in contact with the material. Fig. 4.18 illustrates the four-point probe setup with circuit resistances. The contact resistance $R_{C}$ is about several hundred to a thousand times higher than the resistance of the sample which is noted by $R_{s}$. And the voltmeter only measures the voltage drop value between 2 
and 3 through which the current generated by 1 and 2 flows. Hence, by separating of voltage and current electrodes four probes method eliminates measurement errors due to the probe resistance, the spreading resistance under each probe, and the contact resistance between each metal probe and the measured material.

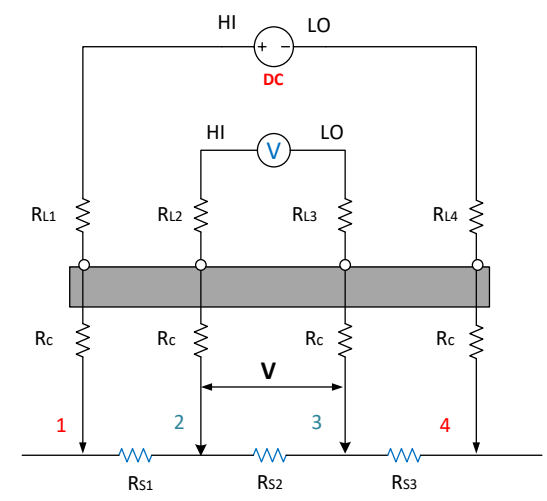

Figure 4.18: Four-point measurement of resistance between voltage sense connections 2 and 3 . Current is supplied via force connections 1 and 4.

In order to measure the bulk resistivity of the deposited contact easily, a structure with small $1 \mathrm{~mm} \times 1 \mathrm{~mm}$ square pads which are added to the ends of the fingers to improve probe-finger contacting in electrical characterization (see Fig. 4.19) is designed in the tests. The resistance in the pad doesn't affect the measurement.

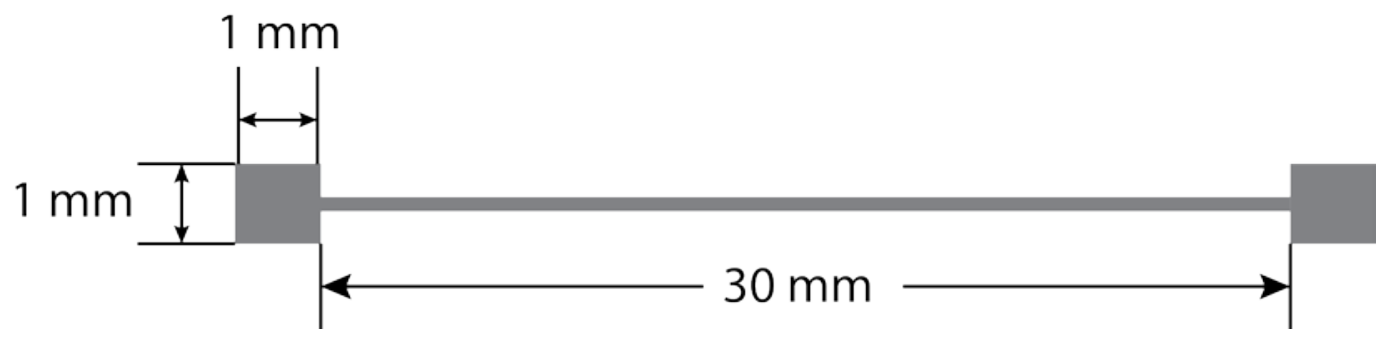

Figure 4.19: Finger structure for bulk resistivity measurement.

The resistance of finger bulk resistivity is measured using KEITHLEY Model 2400 SourceMeter (see Fig. 4.20). In this method, two contacts probe 1 and 2 of the measuring device are placed at one square pad of the finger $(x=0 \mathrm{~mm})$ with a voltage for causing a current to flow, while the other two probes 3 and 4 are placed at the opposite pad $(x=33$ 
$\mathrm{mm}$ ) to measure the voltage difference between the pads. Different voltages (e.g. from $1 \mathrm{~V}$ to $1 \mathrm{~V}$ in $0.04 \mathrm{~V}$ steps) are applied to measure the relative currents. By plotting a V-I curve and performing a linear fit, the ohmic behaviour, $I=V / R$, can be assumed by the slope of the linear fit, which is the inverse of the resistance along the finger.

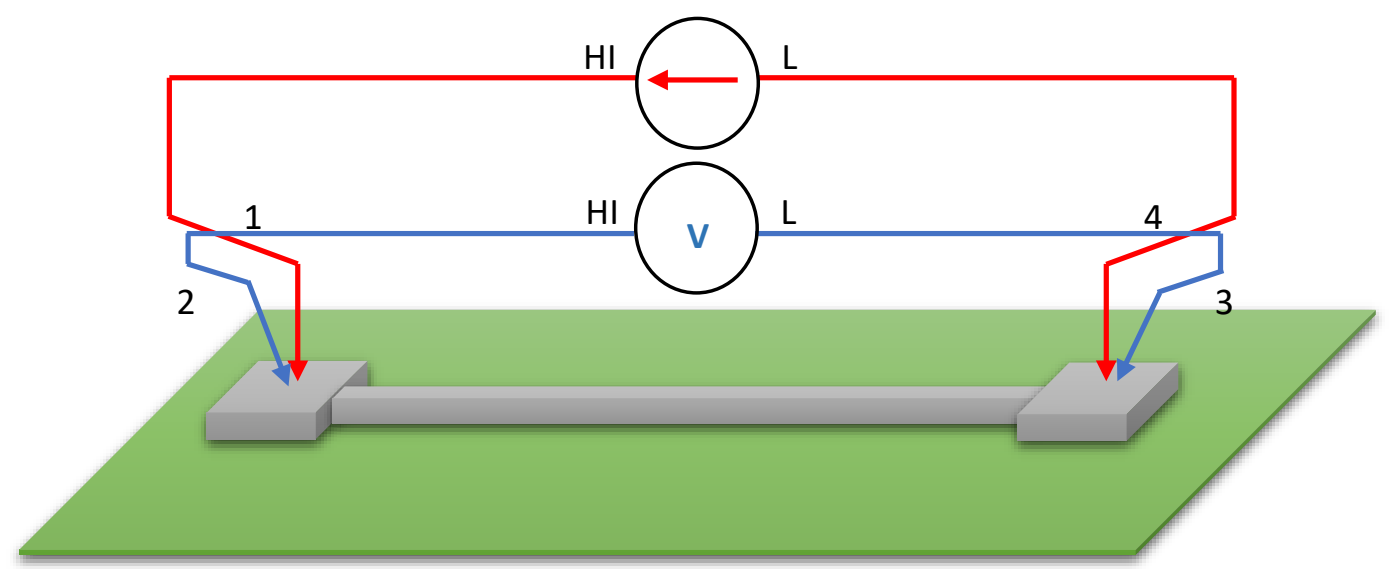

Figure 4.20: Diagram of Four-point probe method by using KEITHLEY Model 2400 SourceMeter.

The bulk resistivity can be obtained by multiplying the resistance by the cross-section area of the body of the finger (i.e. without the cross-section area of the square pads) and divide it by its length. Since the cross-sectional area along the line are not uniform and the measurement data are dispersed, 5 measurements of the cross-sectional profiles of a deposited line was taken by using confocal microscopy for calculating an average cross sectional area (see Fig. 4.21). Each cross-sectional area can be obtained by integrating numerically the extracted profile data by OriginLab or MATLAB using trapezoid rule. 


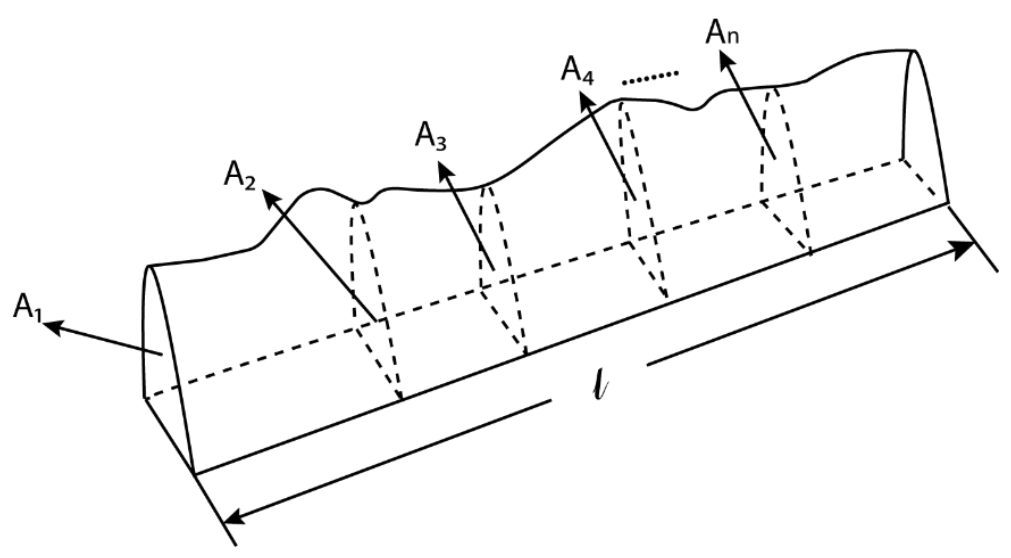

Figure 4.21: Diagram of bulk resistivity calculation method.

From the bulk resistivity equation, we know

$$
R=\rho \frac{l}{A}
$$

\subsubsection{Busbar bulk resistivity measurement}

The busbar structure designed for measuring the resistivity is illustrated in Fig. 4.22. The four-point probe method is also used here for busbar bulk resistivity measurement. The testing procedure is the same as in finger bulk resistivity measurement.

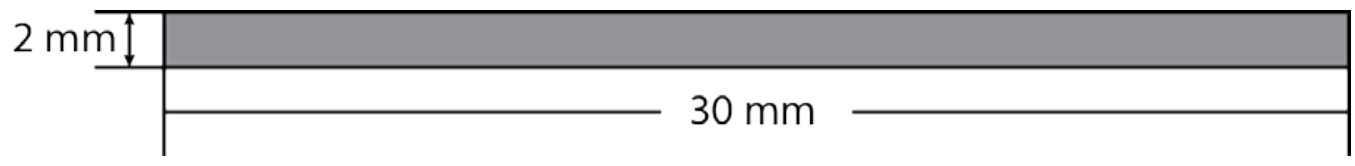

Figure 4.22: Diagram of busbar structure for bulk resistivity measurement.

\subsubsection{Specific contact resistivity measurement}

Specific contact resistivity $\rho_{c}$ is a very useful electrical parameter for characterizing the ohmic contacts of various sizes since it is independent of the contact area. Theoretically 
it is defined as the barrier resisting the current flow in the interfacial which under zero applied bias is given by

$$
\rho_{c}=\left.\frac{\partial V}{\partial J}\right|_{V=0}
$$

where $V$ is the applied voltage across the junction and $J$ is the current density across the metal-semiconductor interface.

It can be also defined as the resistance of a unit area of the metal-semiconductor interfacial layer as

$$
\rho_{c}=\left.\frac{\partial V}{\partial J}\right|_{A \rightarrow 0}
$$

Specific contact resistivity is determined by the measured contact resistance. If consider a small region in the vicinity of the contact

$$
R_{c}=\rho^{\prime} \frac{\Delta x}{A_{C}}
$$

where $A_{C}$ is the contact area, $\rho^{\prime}$ is the material resistivity,

$$
\rho_{c}=\lim _{\Delta x \rightarrow 0}\left(\rho^{\prime} \Delta x\right)=R_{C} A_{C}
$$

The specific contact resistivity has unit of $\Omega \cdot \mathrm{cm}^{2}$. Typical values range from $10^{-3}$ to $10^{-8} \Omega \cdot \mathrm{cm}^{2}$. 
Now consider the two contacts geometry shown in Fig. 4.23. The measured total resistance consists three components

$$
R_{T}=2 R_{m}+2 R_{c}+R_{\text {semi }}
$$

where $R_{m}$ is the resistance of the contact metal, $R_{C}$ is the metal/semiconductor interface contact, and $R_{\text {semi }}$ is the semiconductor resistance between two pads. Since in most cases, $R_{c} \gg R_{m}$, and $R_{s e m i}=R_{s h} L / Z$, Equation (4.14) becomes

$$
\begin{aligned}
R_{T} & =R_{\text {semi }}+2 R_{c} \\
& =R_{\text {sh }} \frac{L}{Z}+2 R_{c}
\end{aligned}
$$

As current flows from semiconductor to the metal contact, it passes through the resistance $\rho_{c}$ and $R_{s h}$, selecting the path of least resistance. The transfer length $L_{T}$ is defined as the " $1 / e$ " distance of the voltage curve, which can be regarded as the distance over which most of current transfer from one material to the other.

$$
L_{T}=\sqrt{\rho_{c} / R_{s h}}
$$

The contact resistance $R_{c}$ is usually referred to the contact front resistance at which $\mathrm{V}$ is measured between two contact at $x=0$,

$$
R_{c}=R_{c f}=\frac{\sqrt{R_{s h} \rho_{c}}}{Z} \operatorname{coth}\left(\frac{L}{L_{T}}\right)=\frac{\rho_{c}}{L_{T} Z} \operatorname{coth}\left(\frac{L}{L_{T}}\right)
$$




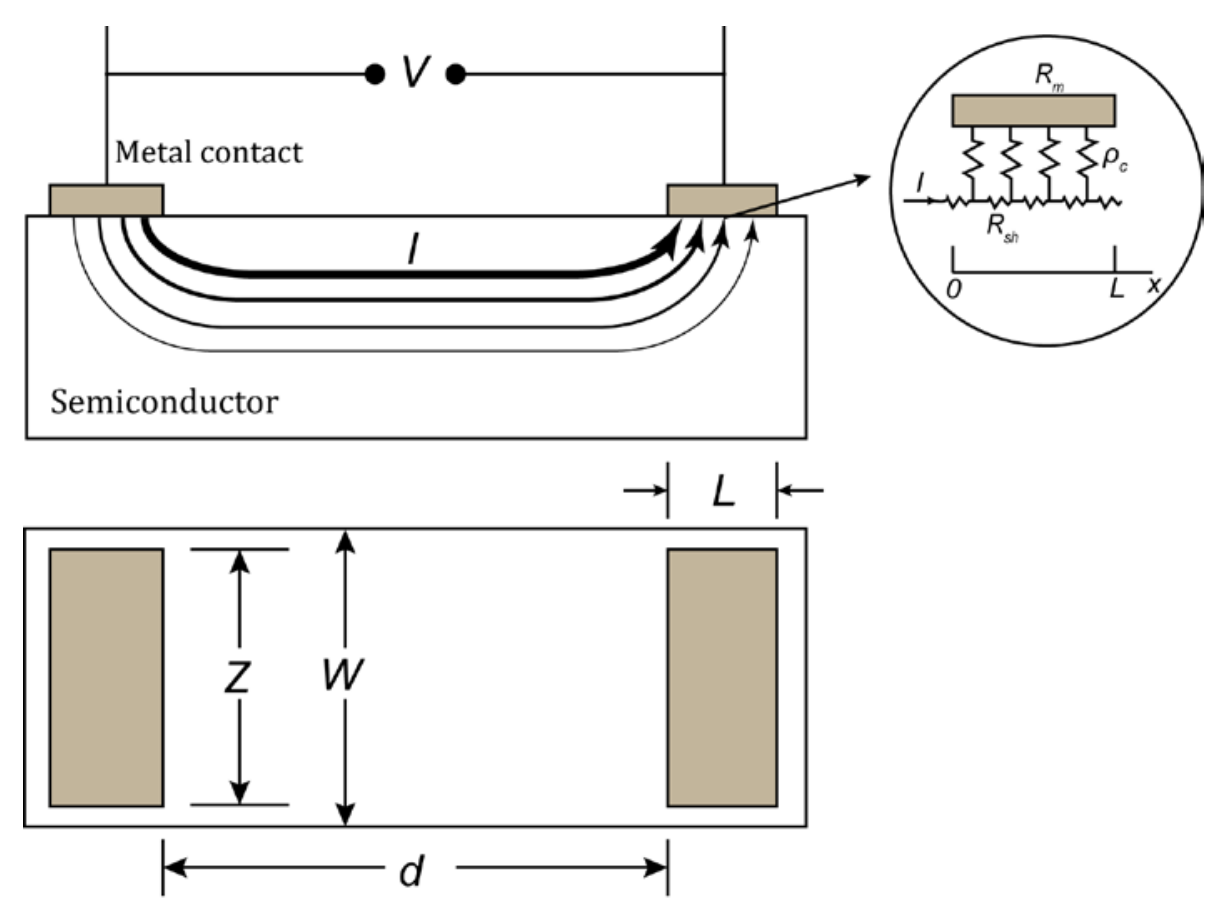

Figure 4.23: Two contact resistance structure in cross section and top view.

The contact resistance can be simplified according to the relationship between the physical length $L$ and the transfer length $\left(L_{T}\right)$. For $L \leq 0.5 L_{T}$ or $L_{T} \geq 2 L$, $\operatorname{coth}\left(L / L_{T}\right) \approx L_{T} / L$, then

$$
R_{c} \approx \frac{\rho_{c}}{L Z}
$$

And for $\mathrm{L} \geq 1.5 L_{T}, \operatorname{coth}\left(L / L_{T}\right) \approx 1$ and

$$
R_{c} \approx \frac{\rho_{c}}{L_{T} Z}
$$

In the first case, the effective contact area is the physical contact area. However, in the second case, the effective area can be smaller than the actual contact area. That is to 
say, the effective contact area can be smaller than the actual physical contact area which is determined by the physical length and width of the contact [98].

The TLM test structure was originally proposed by Shockley in 1964 . The specific contact resistivity measurement was performed by the ladder TLM method. As Fig. 4.24 shows, the method is based on a ladder structure with equally spaced pairs of contact. Two large contact pad in the ending serve as the entry and exit of the current. By applying a known current, the potential difference can be measured and plotted linearly as a function of the spaced distance between the one of the large contact and each of the successive narrow contact. And extrapolating the line to zero potential difference, the transfer length can be obtained.

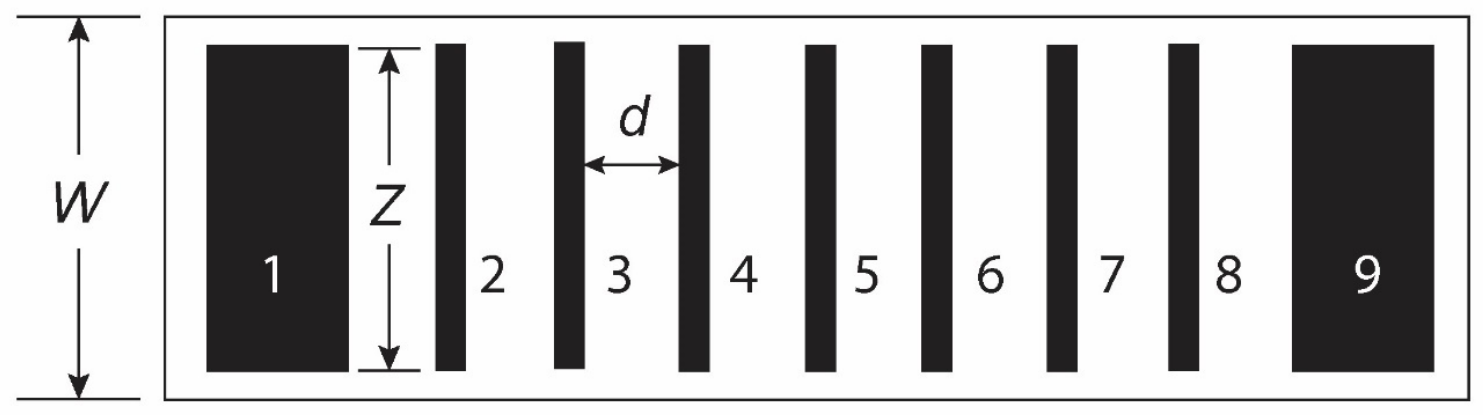

Figure 4.24: Transfer length method test structure proposed by Shockley.

In this structure the current flow may be perturbed by the contacts in between the measured two ending point. For $L \ll L_{T}$, the current flow doesn't penetrate into the metal contact. Hence the contacts in between the ending probes will not affect much the measurement. However, if the narrow width of the contact becomes comparable with the transfer length, saying $L \gg L_{T}$, the current will flow into the contacts. The shunting of the current makes the uncertainty of the potential measurement, which also influences the transfer length determination.

This model was modified and improved by separating each pair of contacts with an uneven distance, which leave only bare semiconductor between the measured probes. 
Hence in the case of $\mathrm{L} \geq 1.5 L_{T}$,

$$
R_{c} \approx \frac{\rho_{c}}{L_{T} Z}=\frac{R_{s h} L_{T}}{Z}
$$

Equation (4.20) can be written as

$$
\begin{aligned}
R_{T} & =R_{\text {semi }}+2 R_{c} \\
& =R_{s h} \frac{L}{Z}+2 \frac{R_{s h} L_{T}}{Z} \\
& =\frac{R_{s h}}{Z}\left(L+2 L_{T}\right)
\end{aligned}
$$

Hence the total resistance $R_{T}$ can be represented as a linear function of the spaced length between two contacts. By plotting the function, the sheet resistance $R_{s h}$ can be derived from the slope, which is equal to $R_{s h} / W$. And by extrapolating back to the horizontal and vertical axis, the transfer length $L_{T}$ and contact resistance $R_{c}$ can be obtained (see Fig. 4.25).
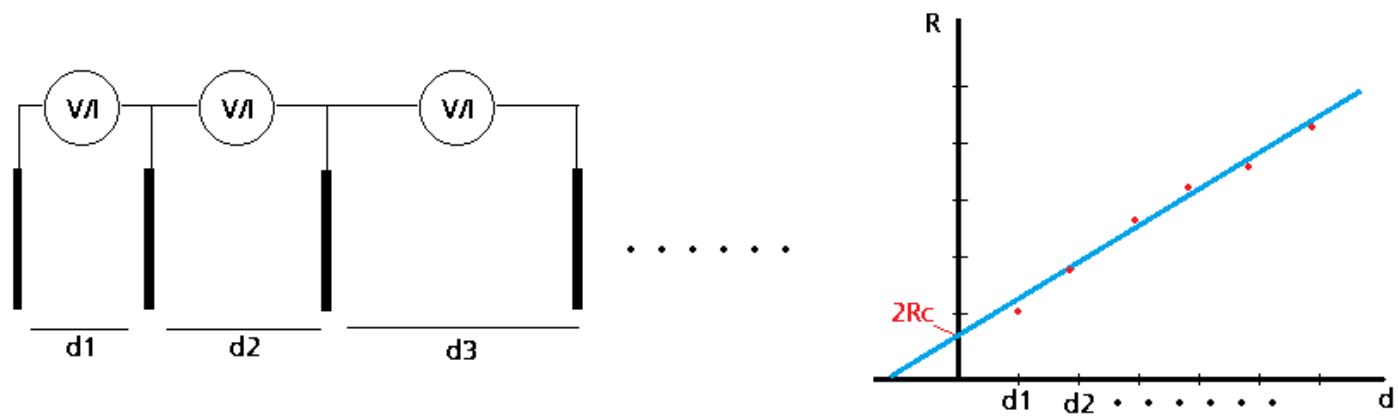

Figure 4.25: The TLM method with $\mathrm{R}_{\mathrm{T}}$ plotted as a function of uneven spacing.

The TLM structure for the LIFT deposited line specific contact resistivity test was illustrated in Fig. 4.26. The structure consists of nine lines, spaced starting from $870 \mu \mathrm{m}$ with spacing increasing by $290 \mu \mathrm{m}$ for each successive finger. A $0.5 \mathrm{~mm} \times 0.5 \mathrm{~mm}$ square pads added to the ends for improving probe-finger contact in the electrical 
characterization. For the sake of making a major assumption that one-dimensional current flow takes place, it is critical to reduce the adjacent area of the finger ends where the current flows around, with $(W-Z) \ll Z$. The LIFT deposited contact length $L$ is supposed to be in the range between $100 \mu \mathrm{m}$ and $200 \mu \mathrm{m}$, so the contact width $Z$ is made of $1 \mathrm{~cm}$ to fulfill the condition that $Z \gg L$ to ensure one-dimensional current flow. The small $L$ value, if designed with $L \ll L_{T}$, can bring the benefit that all the physical contact area of the finger is utilized in transferring the current. After the LIFT deposition of the TLM structure, the laser isolation was performed as near as possible to the ends of the fingers, namely with $W \cong Z$.

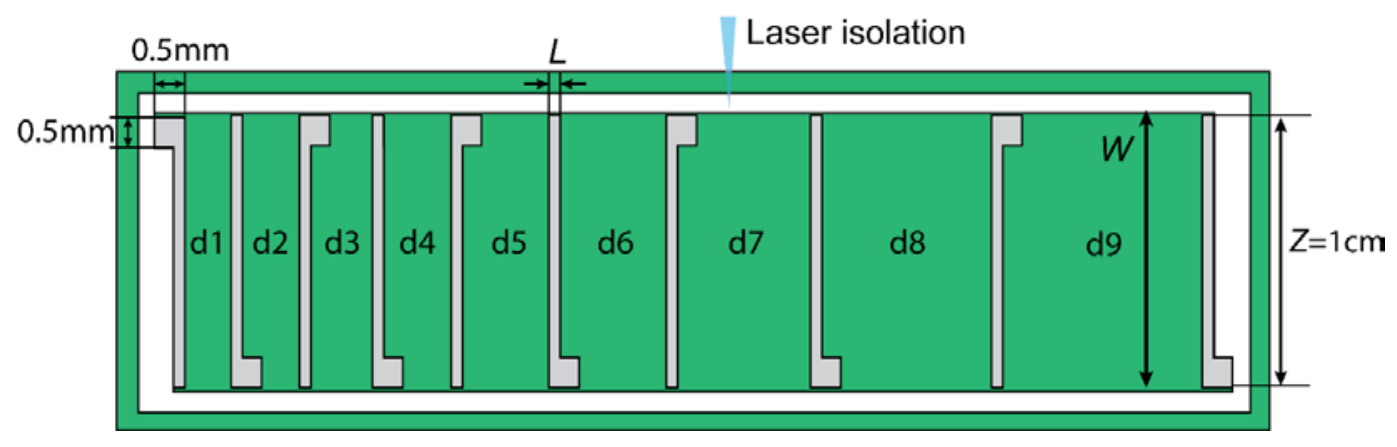

Figure 4.26: Schematic of TLM structure with laser isolation.

The total resistance $R_{T}$ was measured using four-probe method, which is same method mentioned in the bulk resistivity measurement section. Then by comparing transfer length $L_{T}$ with contact length $L$, the specific contact resistance can be obtained by

$$
\begin{gathered}
\rho_{c}=R_{c} L Z ; \text { if } \mathrm{L} \geq 1.5 L_{T} \\
\rho_{c}=R_{c} L_{T} Z ; \text { if } \mathrm{L} \leq 0.5 L_{T} \\
\rho_{c}=R_{c} L_{T} Z \tanh \left(\frac{L}{L_{T}}\right) ; \text { if } 0.5 L_{T} \ll \mathrm{L} \leq 1.5 L_{T}
\end{gathered}
$$




\subsection{Solar cell characterization}

The solar cell device characterization (IV measurement) has been performed with the four probes method with a system composed by a computer-programmed Keithley 2420 source/meter and a Solar simulator (Newport Oriel class A with research Arc Lamp Housing Model 67005) for providing the illumination. The fundamental principle of this method is discussed in the section 4.6. The light source was calibrated with a calibrated reference solar cell (see Fig. 4.28) and meter (Newport ORIEL 91150V) for simulating the irradiance in sun unit, one sun condition, which corresponds to standard illumination at Airmass 1.5 Global Reference (AM 1.5G) (100 mW. $\mathrm{cm}^{-2}$ ) (see Fig. 4.26). In this work, the solar cell characterization has also been cooperated with Universidad Politécnica de Catalonia for a comparison. The solar cell characterization method used there is the same four-point probe method with Keithley 2420 source/meter and a solar simulator (Newport ORIEL 94021A) under 1 sun illumination condition $\left(100 \mathrm{~mW} \cdot \mathrm{cm}^{-2}\right)$. The schematic of the solar cell characterization method is depicted in Fig. 4.29.

By set the swept voltage in the source/meter from 0 to $2 \mathrm{~V}$, four main photovoltaic parameters, namely short-circuit current $\left(\mathrm{I}_{\mathrm{sc}}\right)$, open circuit voltage $\left(\mathrm{V}_{\mathrm{oc}}\right)$, Fill Factor $(\mathrm{FF})$ and cell efficiency $(\eta)$, which characterize the performance of the solar cell device can be determined from the measured IV curve data of the illuminated solar cell device. The short circuit $\left(\mathrm{I}_{\mathrm{sc}}\right)$, open voltage $\left(\mathrm{V}_{\mathrm{oc}}\right)$, maximum power point $\left(P_{\max }\right)$ can be directly obtained from the IV curve data (see Fig. 4.27).

The fill factor $(F F)$, which is an essential parameter of the measure of quality of the

solar cell, can calculated by comparing the maximum power to the theoretical power that would be output at the open circuit voltage and short circuit current together (see Equation 4.25 below).

$$
F F=\frac{I_{m p} \cdot V_{m p}}{I_{s c} \cdot V_{o c}} \times 100
$$




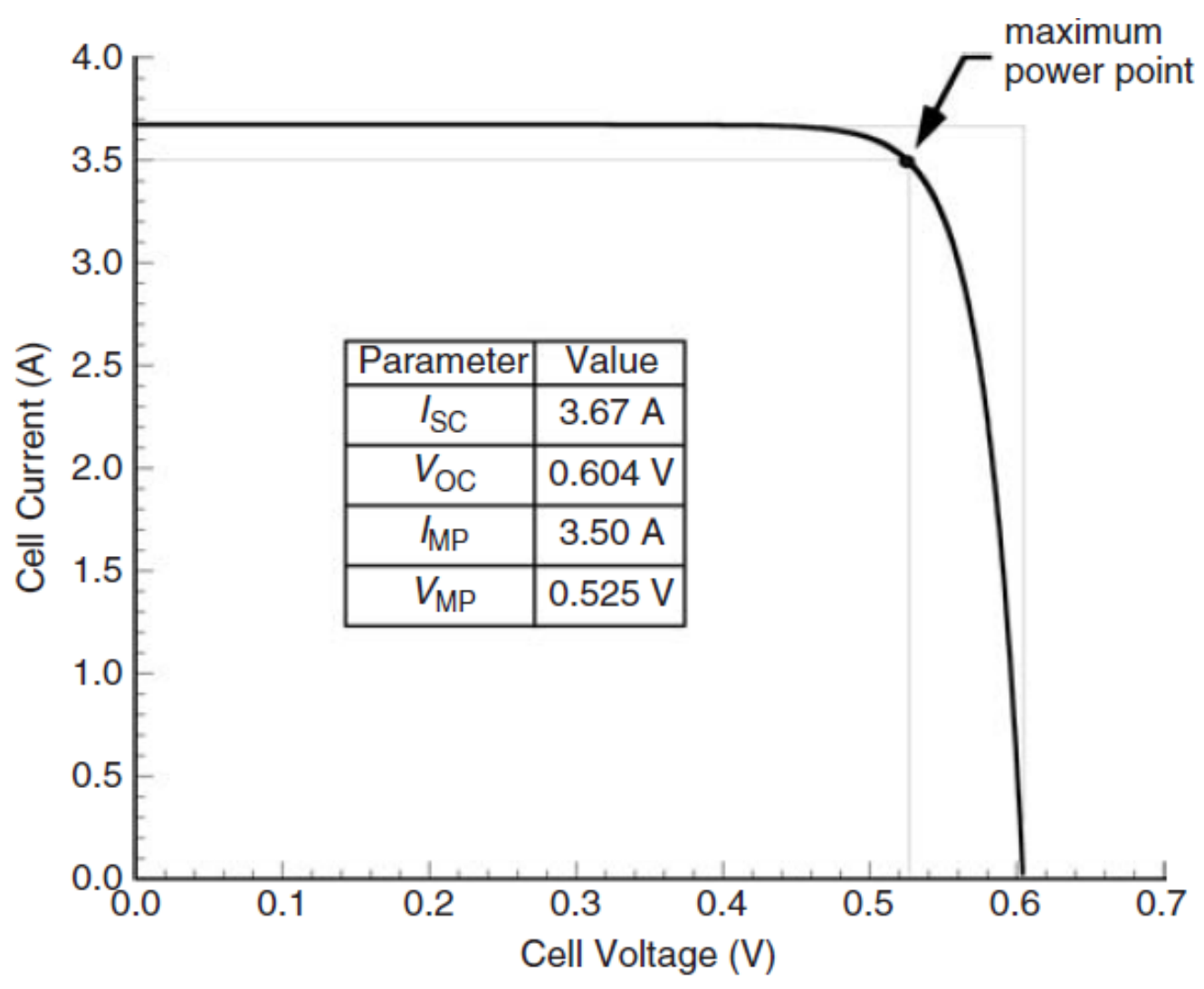

Figure 4.27: An example of IV curves characteristic calculation for solar cell [20].

Cell efficiency $(\eta)$ is the ratio fo the electrical power output $P_{\text {out }}$, compared to the solar power input, $P_{\text {in }} . P_{\text {out }}$ can be taken to the $P_{\max }$. By dividing the illuminated area $\left(2.2 \times 1.1 \mathrm{~cm}^{2}\right)$ in both numerator and denominator, $I_{m p}$ is replaced by current density $\left(U_{m p}\right)$ and $P_{i n}$ is replaced by the standard input irradiance $\left(E_{i n}\right)$ of one-sun condition in the measurement which is $100 \mathrm{~mW} \cdot \mathrm{cm}^{-2}$ and, see Equation 4.26 below,

$$
\eta=\frac{P_{\text {max }}}{P_{\text {in }}}=\frac{I_{m p} \cdot V_{m p}}{P_{\text {in }}} \times 100=\frac{J_{m p} \cdot V_{m p}}{E_{\text {in }}} \times 100
$$




\section{Curve Newport 10510-0050}

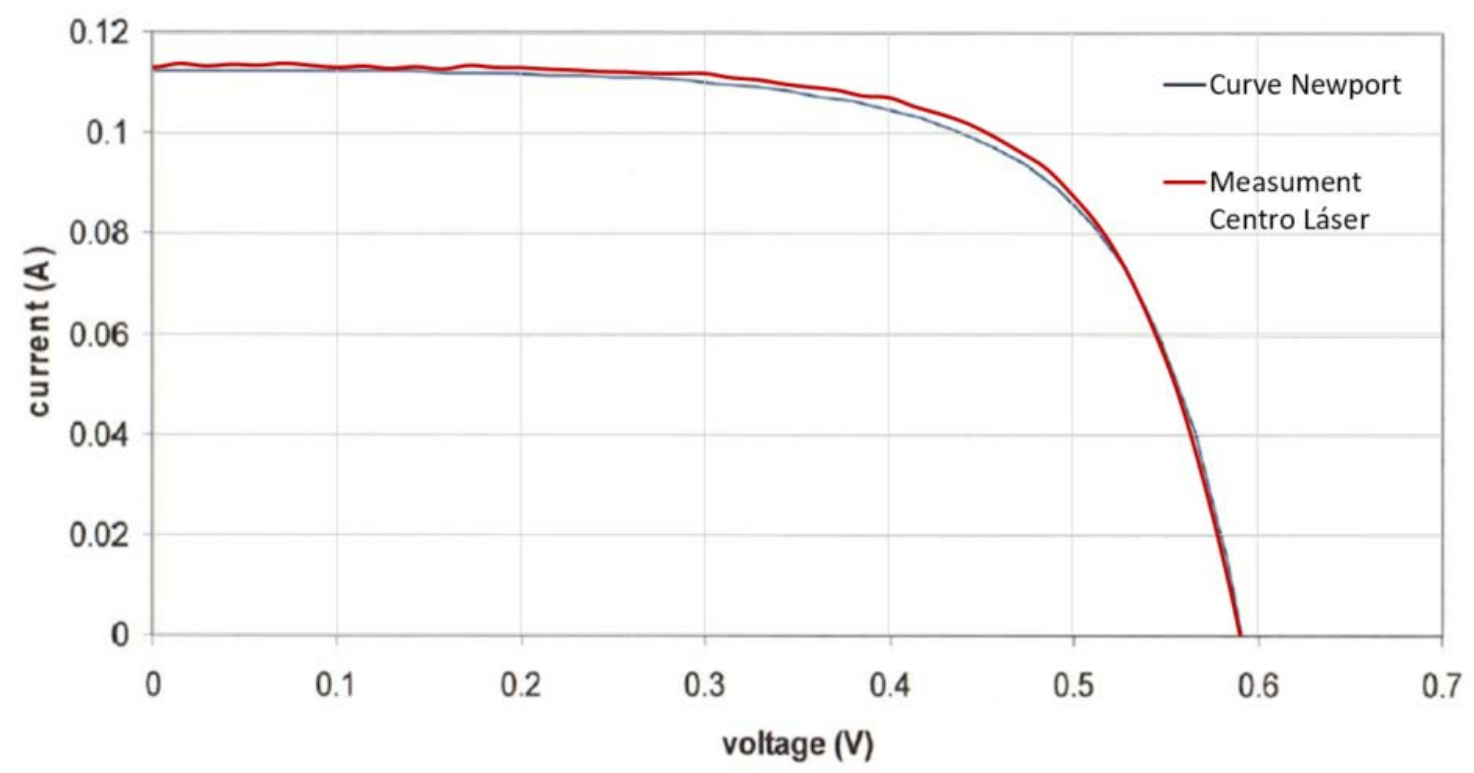

Figure 4.28: IV curves obtained by using a reference solar cell for the standard illumination calibration of the solar simulator in the laboratory.
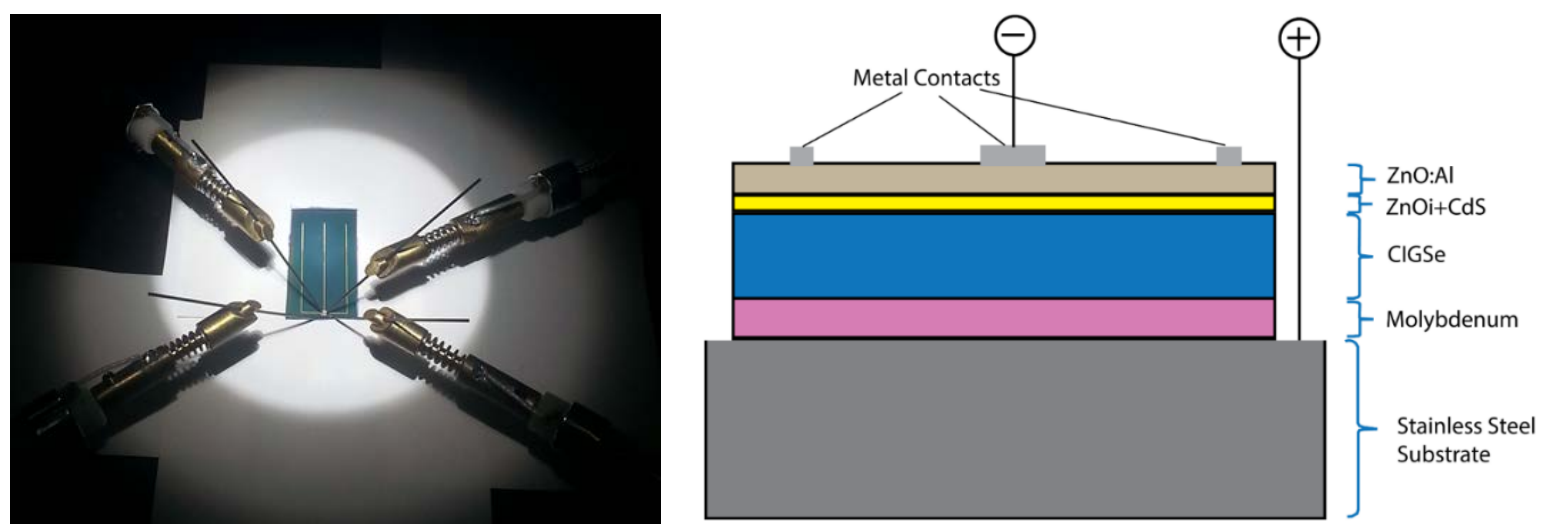

Figure 4.29: Schematic of CIGS solar cell measurement with four probes 


\section{Nanosecond LIFT results}

\subsection{Voxel printing by single pulse}

In this section, a study of individual dots of silver paste printed by laser-induced forward transfer (LIFT) is performed using the ns-pulsed DPSS Nd:YVO 4 laser with a wavelength of $532 \mathrm{~nm}$. Single Gaussian laser pulses with spot size of $25 \mu \mathrm{m}$, which is on the order of the donor film thickness, are employed to print voxels with large aspect ratios. The morphology of the printed voxel on the acceptor substrate and the holes left in the film on the donor substrate in pairs are characterized by scanning confocal microscopy and discussed in terms of the transfer mechanisms. In addition, a time-resolved imaging study is presented to enable a detailed explanation of the transfer dynamics during the transfer with respect to the process parameters, mainly film thickness and pulse energy. It explained the influence of process parameters on the structure of transferred dots phenomenologically and analytically.

Depending on the experimental conditions, different transfer regimes with similarities to those reported for LIFT of Newtonian fluids and Nano-pastes were observed. However, the multiphase and non-Newtonian rheology and thicker films used lead to noticeable differences. In addition, a new transfer mechanism is demonstrated. When the paste film is thick enough and the energy is optimum, the protruding jet impacts the acceptor substrate to form a continuous and stable pillar, which remains connected to the donor substrate, and broken once the donor substrate is taken out. This mechanism leads to transferred dots with large aspect ratios, which is desirable for the printing of contacts of solar cells.

\subsubsection{Morphological characterization of printed voxel}

In this voxel printing experiment, the laser employed is the ns-pulsed DPSS Nd:YVO 4 green laser (Explore, 532nm) and the donor film involved is the commercial micro-sized silver paste (DuPont Solamet PV17F). The donor preparation and experimental setup is descripted in the chapter 4 . The dotting experiment was implemented with a matrix in 
which the 5 dots were separated $150 \mu \mathrm{m}$ apart and fired in each group with the same laser pulse energy varied from $0.5 \mu \mathrm{J}$ to $35.3 \mu \mathrm{J}$, corresponding to the peak fluences from 0.2 to $14.4 \mathrm{~J} / \mathrm{cm}^{2}$ (see Fig. 5.1). Then the donor was removed from the acceptor substrate leaving transferred silver paste dots. The morphology of the printed voxels and holes in the donor substrate were then studied using confocal microscopy.

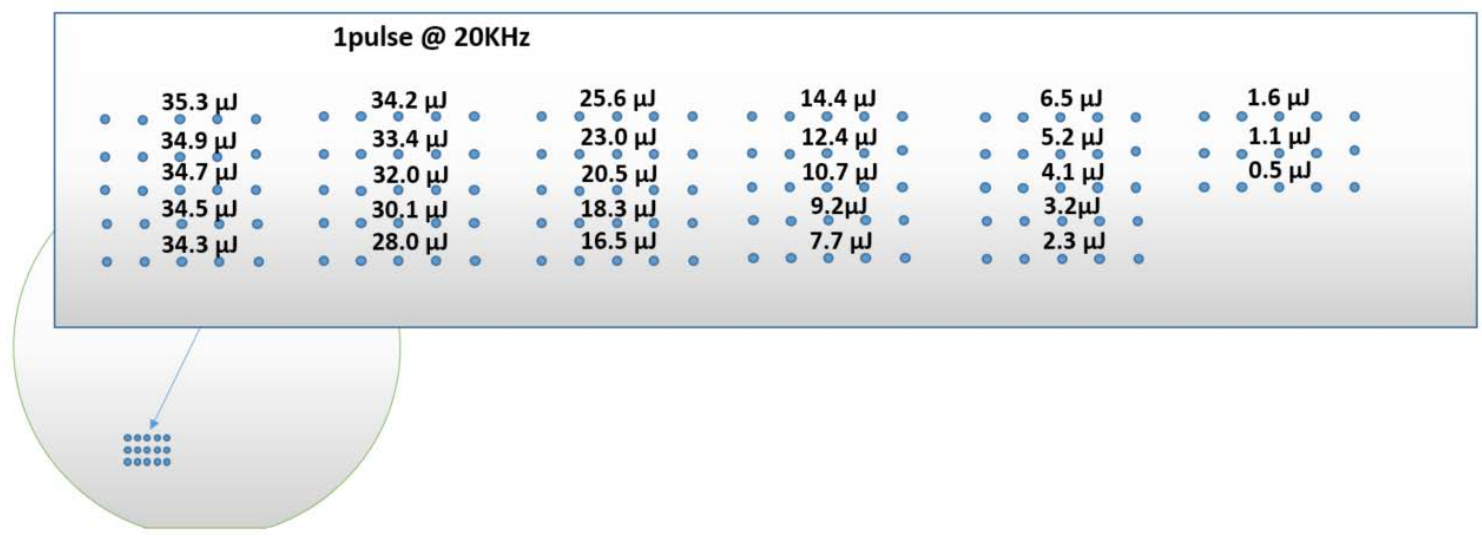

Figure 5.1: Schematic of the voxel printing matrix.

Microscope images of voxels printed with single laser pulse using four different fluences and the two donor thicknesses are represented Fig. 5.2. Below the lowest fluence $\left(0.6 \mathrm{~J} / \mathrm{cm}^{2}\right)$, there is nothing transferred for the $50-\mu \mathrm{m}$-thick donor, while small clusters of paste formed on the acceptor substrate with $30-\mu \mathrm{m}$-thick donor. It indicates that there is an energy transfer threshold depending on the donor thickness. This is consistent with previous studies of LIFT using Newtonian fluids [17, 66, 99, 100], and can be understood by the observation that more material must be accelerated in the case of a thicker donor, requiring more energy. When the pulse energy increases there is paste transfer using both thicknesses, although in the case of the $30 \mu \mathrm{m}$-thick donor films the voxel always exhibits splashing, with finer and more numerous droplets at higher energies. The $50 \mu \mathrm{m}-$ thick donor leads to concrete transfer, with a single paste dot that shows no splashing or disaggregation of the voxel. The size of the dot increases as energy increases. At the largest fluence $\left(5.9 \mathrm{~J} / \mathrm{cm}^{2}\right)$, the concrete dot is surrounded by clearer debris that could be due to organic residue. 


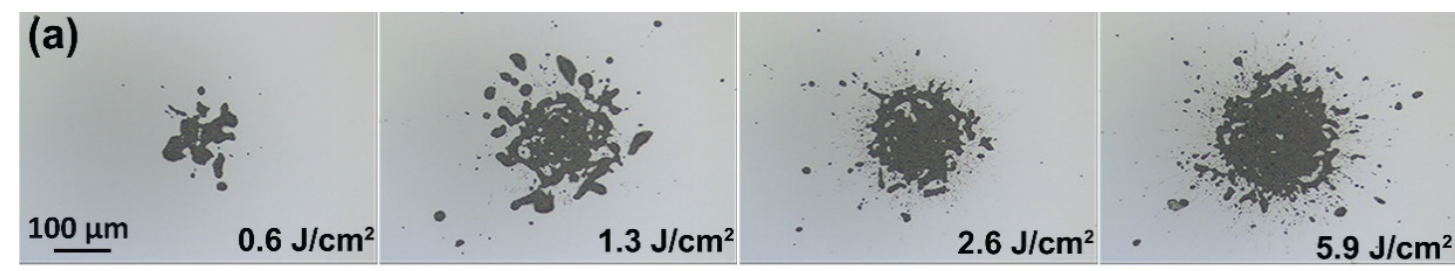

(b)

\section{$0.6 \mathrm{~J} / \mathrm{cm}^{2}$}

$1.3 \mathrm{~J} / \mathrm{cm}^{2}$

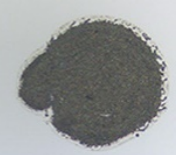

$2.6 \mathrm{~J} / \mathrm{cm}^{2}$

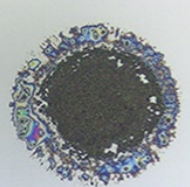

$5.9 \mathrm{~J} / \mathrm{cm}^{2}$

Figure 5.2: Microscope images of LIFT dots transferred using single shots of a ns laser with fluence varying from 0.6 to $5.9 \mathrm{~J} / \mathrm{cm}^{2}$ with two different donor thicknesses: (a) $30 \mu \mathrm{m}$ and (b) $50 \mu \mathrm{m}[19]$.

The morphology of the voxels on the acceptor substrate are characterized using confocal microscopy (Leica DCM 3D). And due to the high viscosity of the silver paste holes will be left in the film on the donor substrate and can be also studied by confocal microscopy. Fig. 5.3 shows the false color height map of both donor (Fig. 5.3a) and acceptor (Fig. 5.3c) substrates for the two thicknesses. It also shows the corresponding cross-section profiles (Fig. 5.3b). The reference straight horizontal line in the profiles indicates the interface between the silver paste and the glass slide.

For the donor substrates, the holes reached the glass slide, with no silver paste remaining in the bottom of the hole in either case. The hole left in the thicker donor is smaller in diameter and the inside wall is steeper compared to the thinner-donor case. A crustal annular bulge formed surrounding the crater is observed in both cases. Furthermore, the width and the shape of the holes are congruent with those of the transferred voxels. Widths of holes and voxels are similar and on the order of $100 \mu \mathrm{m}$, much larger than the laser beam spot. However, there is a large difference in the height of the voxels and the depth of the hole, which indicates that a significant portion of the paste affected by the laser pulse is not transferred but displaced to the border of the hole. 


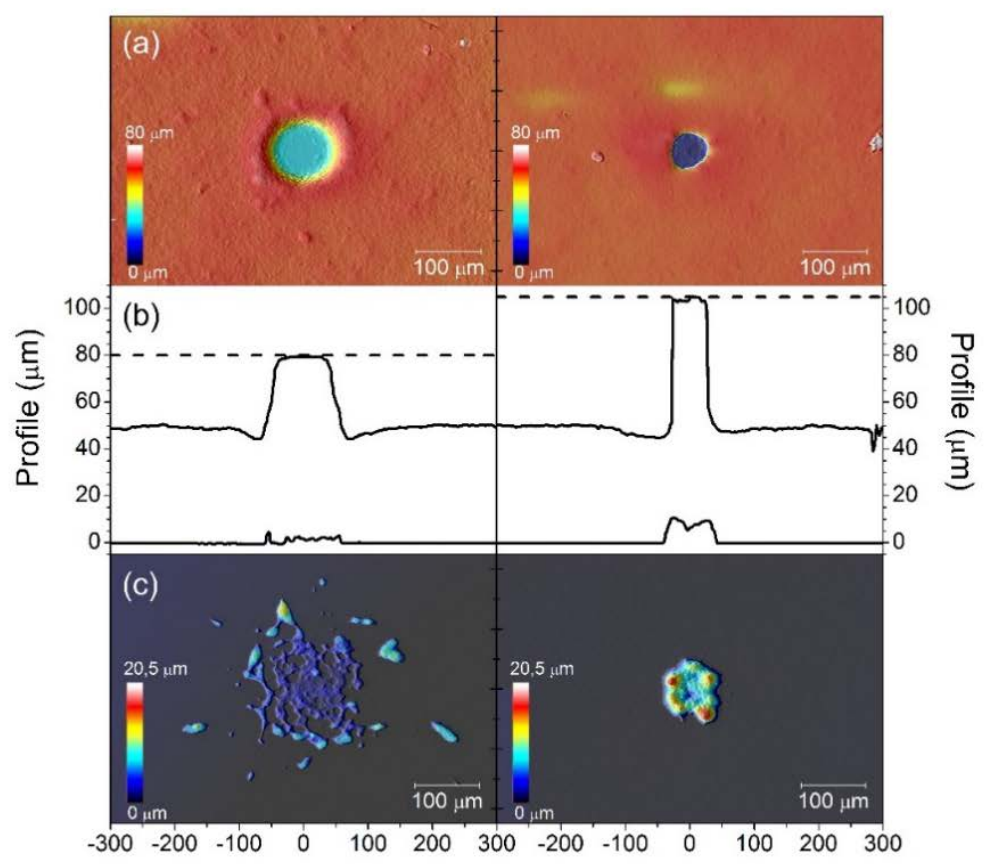

Figure 5.3: Confocal microscope images of (a) donor films and (c) transferred dots from $1.3 \mathrm{~J} / \mathrm{cm}^{2}$ laser pulses with films of thickness (left) $30 \mu \mathrm{m}$ and (right) $50 \mu \mathrm{m}$. Profiles of both donor and dot are shown in (b) [19].

Figure 5.4 represents the voxel size (height, width and aspect ratio) as a function of the laser peak fluence. Since the aim of this work is to use LIFT to metalize devices, the aspect ratio, calculated as the ratio of height to width, can be considered a suitable figureof-merit of the process: higher widths result in lower electrical resistances of transferred lines, while lower widths lead to a smaller area occupied by the line in the final device. The width of the voxel is defined as the diameter of the compact circular-like region excluding the surrounding scattered clusters, and the height is defined as the average value of the measured profile. Each data point in Fig. 5.4 represents the average of 10 measurements, with error bars denoting one standard deviation. The width of the dots is difficult to define in the low-energy regime for the 30- $\mu \mathrm{m}$-thick donor (between 0.6 and $2.0 \mathrm{~J} / \mathrm{cm}^{2}$ ), where the dots are made up of large clumps of paste (Figure 5.2a). In the case of the $30-\mu \mathrm{m}$-thick donor, the height of the voxels remains almost constant as energy increases, while in the case of the 50 - $\mu$ m-thick donor, the height decreases with energy. 
Using both donor thicknesses, the width of the voxels increases as a function of laser fluence in a range between 0.6 and $7.4 \mathrm{~J} / \mathrm{cm}^{2}$. Thus, the decrease of aspect ratio with fluence is revealed as a common trend. Using higher laser fluences (beyond $8 \mathrm{~J} / \mathrm{cm}^{2}$ ), which are not shown, the voxel size has a downward trend and finally saturates. This phenomenon could be due to the absorption of the focused pulse in the glass substrate exceeding the damage threshold of glass. In any case, this fluence regime is not suitable for transferring voxels with large aspect ratios, so it is not considered. The optimal transferred voxel with the largest aspect ratio is obtained using the $50-\mu \mathrm{m}$-thick donor and the threshold fluence for transfer. As the energy increases, more fluid can be moved until there is no more left to move. If the energy keeps increasing the pressure is expected to be higher and the paste will have more speed and will spread more increasing the width and reducing the height.

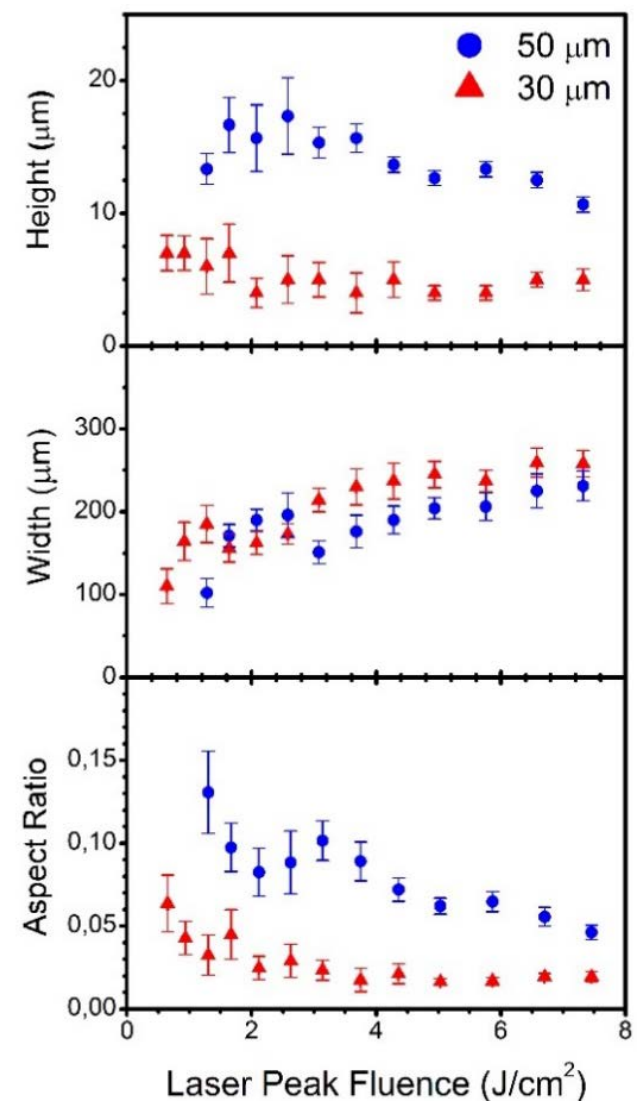

Figure 5.4: Height, width and aspect ratio of the transferred dots as a function of laser peak fluence with respect to two different paste thicknesses (30 $\mu \mathrm{m}$ and $50 \mu \mathrm{m})$ [19]. 
The evolution of the transferred dot morphology as a function of laser pulse energy indicates a classification of four distinctive transference regimes, which is shown in the two series of representative images of transferred dots with respect to two different silver paste thickness. To summarize, the experimental results on voxel transfer show that four different regimes can be considered depending on the laser peak fluence and the donor thickness:

- No transfer. When using laser fluences below a threshold value that depends on the donor thickness, no transfer is observed.

- Cluster transfer. In the thin-donor case at low fluences, the voxel consists of non-continuous clusters of paste. The voxel width increases as fluence increases, while the height remains small.

- Explosive transfer. At high laser fluences, the transfer resembles a splash, with larger and relatively uniform clusters of paste in the center of the spot and a large quantity of small droplets of paste further from the center. The aspect ratio is very small (small height and large width).

- Concrete transfer. In the thick-donor case, a single, concrete dot of paste is observed for the whole range of fluences studied. As the laser fluence increases, the height decreases and the width increases, leading to large aspect ratios for energies just above the transfer threshold. Thus, this is the mechanism of technological interest, because it allows for the successful transfer of large aspect ratio and well-formed lines of paste using LIFT.

\subsubsection{Time-resolved imaging experiment}

To gain insight into the transfer mechanisms responsible for the various regimes observed earlier, side-view images of the LIFT process were obtained with time-resolved optical microscopy. The experimental setup used is similar to that of the previous section, with differences highlighted here. Pulses from a frequency-tripled $\mathrm{Nd}: \mathrm{YVO}_{4}$ laser (Coherent AVIA) emitting at $355 \mathrm{~nm}$ with a pulse duration of $20 \mathrm{~ns}$ are focused by a 10x laser objective $(N A=0.25)$ down to a beam waist of about $15 \mu \mathrm{m}$ to initiate transfer of silver 
paste. Time-resolved images of the transfer from a side view are obtained with a microscope (InfiniTube with a Mitutoyo 50x Long-WD Objective) attached to a CCD camera (SPOT Insight IN1820). Illumination is provided by strobing a 25-ns plasmadischarge flash lamp (HSPS Nanolite) after a specified time delay relative to the laser pulse, set by a digital delay generator (SRS DG535) [97].

Although the experimental system used has a different wavelength and spot size, the objective is to find similar transfer conditions to those shown in section 5.1.1. Silver paste used as donor material is absorbent in the two wavelengths. Therefore, the physics underlying the process should be the same in both cases, apart from minor differences in the particular fluence thresholds. In section 5.1.2.1, the acceptor substrate is not included in order to make illumination easier, following previous time-resolved imaging studies [66, 101]. Later, in section 5.1.2.2, an acceptor is included to investigate its effect on transfer. The donor assembly is moved with a translation stage after each pulse, and a top-down camera (EO-5012C) is used to ensure pulses are fired far enough away from previous holes in the donor film.

\subsubsection{Effects of film thickness and pulse energy}

Sequences of images at increasing time delays are presented for various donor thicknesses and laser pulse energies. Fig. 5.5 shows sequences for a silver-paste donor of thickness $120 \mu \mathrm{m}$ at two laser pulse energies. It is immediately clear that the transfer of silver paste is significantly different from that of Newtonian liquids observed in most timeresolved imaging studies of LIFT $[66,101,102]$; rather than forming a smooth jet that breaks up into droplets, the action of the laser on the silver paste forms a coherent blister shape that then fragments into clumps of paste. At the laser peak fluence of $8.5 \mathrm{~J} / \mathrm{cm}^{2}$ used in Fig. 5.5a, the protrusion fragments into a few clumps, with a larger primary clump propelled away from the donor. Increasing the peak fluence to $23 \mathrm{~J} / \mathrm{cm}^{2}$, the transfer in Fig. 5.5b resembles an explosion, with a more uniform distribution of clumps expanding outward over time. In both cases, a crown-like feature is observed above which the displaced paste remains intact, and below which fragmentation occurs. 

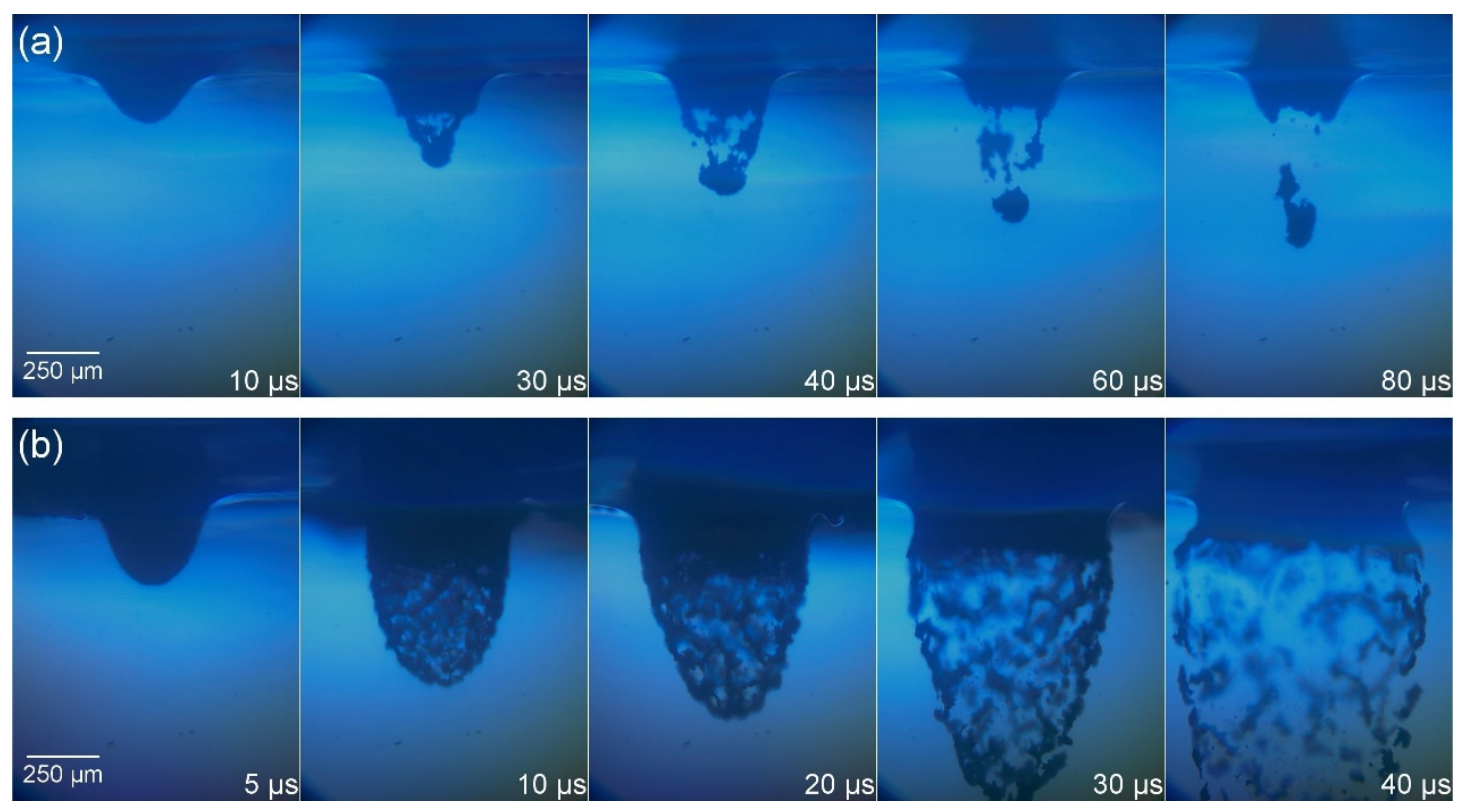

Figure 5.5: Time-resolved images of LIFT of silver paste from a donor film of thickness $120 \mu \mathrm{m}$ using laser fluences of approximately (a) 8.5 and (b) $23 \mathrm{~J} / \mathrm{cm}^{2}$ [19].

Similar mechanisms of transfer are observed for thinner donors. Fig. 5.6 shows sequences of images for three different energies for a donor with a thickness of approximately $60 \mu \mathrm{m}$. The lowest fluence $\left(2.8 \mathrm{~J} / \mathrm{cm}^{2}\right)$ is below the threshold for transfer, resulting in the formation of a stable blister, shown in Fig. 5.6a. This behavior is similar to the blister formation achieved by focusing a laser pulse into a solid polymer film [97, 103] and used for blister-actuated LIFT $[48,67]$. Above the transfer threshold, a regime of transferring a few clumps is again observed for $4.2 \mathrm{~J} / \mathrm{cm}^{2}$ pulses in Fig. $5.6 \mathrm{~b}$, and more explosive transfers are observed at the higher peak fluence $8.5 \mathrm{~J} / \mathrm{cm}^{2}$ in Fig. $5.6 \mathrm{c}$. Interestingly, these laser fluences are about half as large as the corresponding values generating the same behavior in the thick-donor case, and the donor thickness is also half that of the thick-donor case. The time scales for transfer in the thin-donor case are also roughly half that of the thick-donor case. 


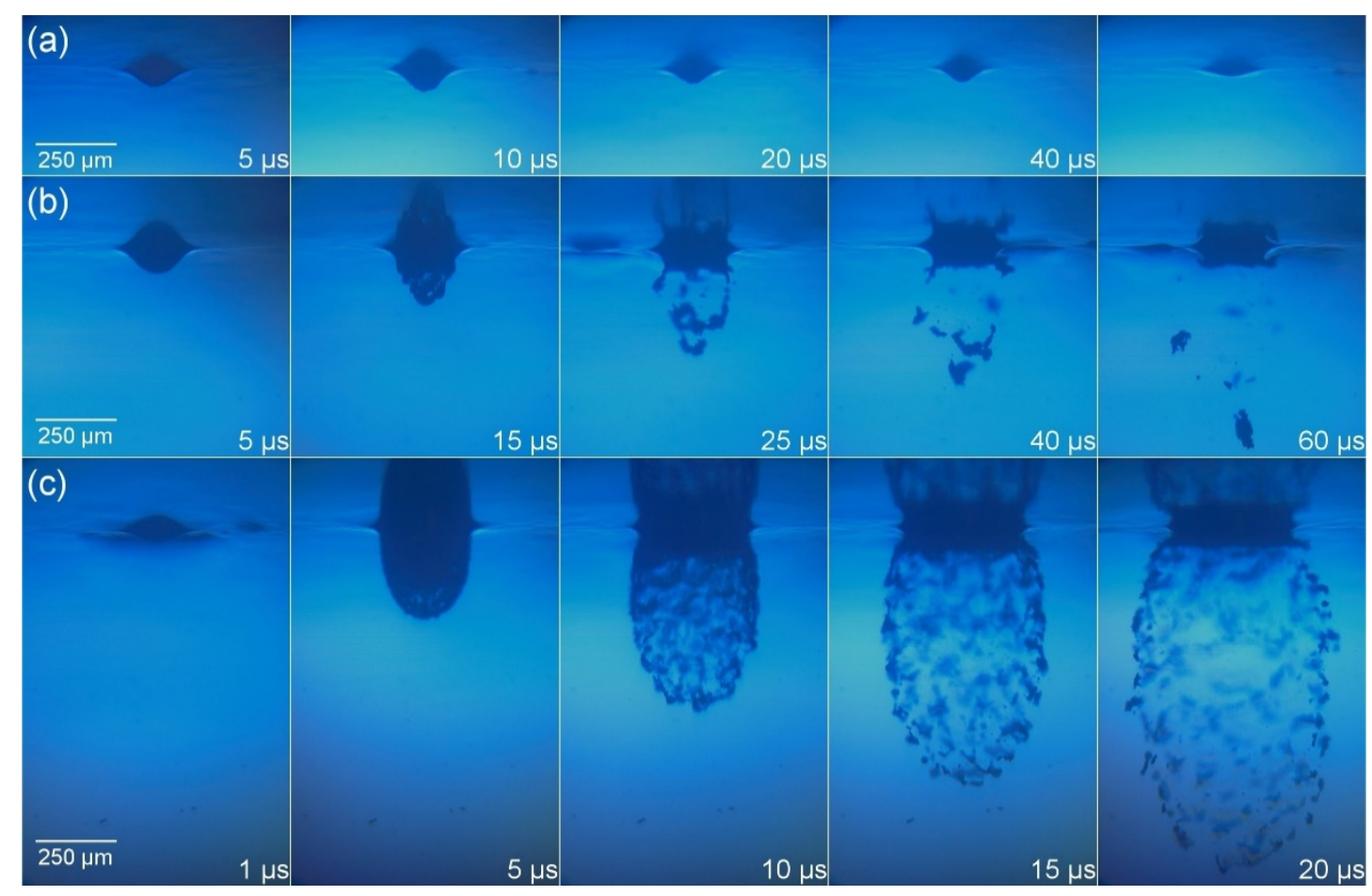

Figure 5.6: Time-resolved images of LIFT of silver paste from a film of thickness $60 \mu \mathrm{m}$ using laser fluences of approximately (a) 2.8 , (b) 4.2 , and (c) $8.5 \mathrm{~J} / \mathrm{cm}^{2}$ [19].

Finally, Fig. 5.7 represents sequences of images for three energies with an even thinner donor of thickness $12 \mu \mathrm{m}$. The laser fluences leading to transfer are much lower $\left(0.6\right.$ and $\left.2.3 \mathrm{~J} / \mathrm{cm}^{2}\right)$ than for thicker donors, and the time scale of the whole process is shorter as well. In this thin-donor case, even the lowest fluence imaged, $0.6 \mathrm{~J} / \mathrm{cm}^{2}$, leads to explosive transfers. The transition from no transfer to a few fragmented clumps to a porous explosion appears to become more compressed with thinner donors.

The observation of transfers with a small number of clumps just above the threshold fluence and more uniform explosions for higher fluences correlates well with the transfer regimes of cluster dot transfer and explosion dot transfer observed earlier, respectively. To observe the concrete dot transfer regime, an acceptor substrate must be included in the imaging setup. 

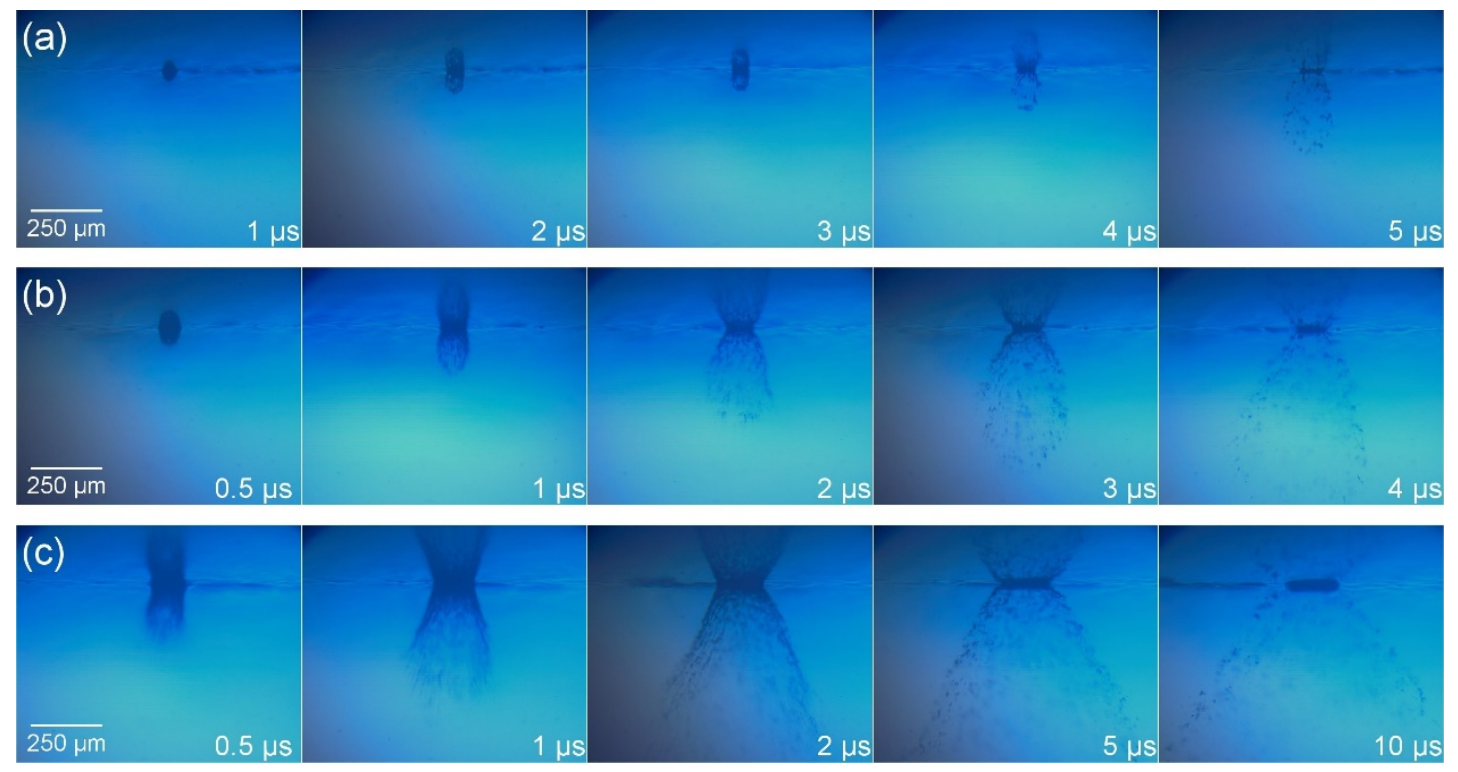

Figure 5.7: Time-resolved images of LIFT of silver paste from a film of thickness $12 \mu \mathrm{m}$ using laser fluences of approximately (a) 0.6 , (b) 2.3 , and (c) $8.5 \mathrm{~J} / \mathrm{cm}^{2}$ [19].

\subsubsection{Influence of an acceptor substrate}

The presence of an acceptor substrate can dramatically affect the transfer, depending on the spacing between the donor and acceptor. For large enough gaps, the transfers will be similar to those imaged in 5.1.2.1, but as the gap gets small enough and of the order of the donor thickness, confinement can play a large role.

Fig. 5.8 shows sequences of images taken with an acceptor substrate approximately $140 \mu \mathrm{m}$ from the donor film for two donor thicknesses and similar laser fluences. First, in Fig. 5.8a, transfers from a donor of thickness $35 \mu \mathrm{m}$ with a peak fluence of $6.8 \mathrm{~J} / \mathrm{cm}^{2}$ are shown to reach the acceptor while exploding, leaving a thin resulting deposit which has splashed to cover a large area. Fig. $5.8 \mathrm{~b}$ shows that for the thicker $60-\mu \mathrm{m}$ donor with 8.4 $\mathrm{J} / \mathrm{cm}^{2}$ transfers, the confinement of the acceptor prevents the paste from exploding, instead forming a stable, connected bridge akin to liquid bridges. The final shape of the voxel is therefore not obtained until the donor substrate is removed from the acceptor. 
The donor thickness and peak fluence of Fig. $5.8 \mathrm{~b}$ are equivalent to the values used to obtain the exploding transfers in Fig. 5.8c, so it is clear that the acceptor substrate plays a crucial role in preventing fragmentation. The donor-thickness dependence is consistent with the trend observed in 5.1.2.1, that pulses of a given energy are more likely to cause explosive transfers as the donor gets thinner.
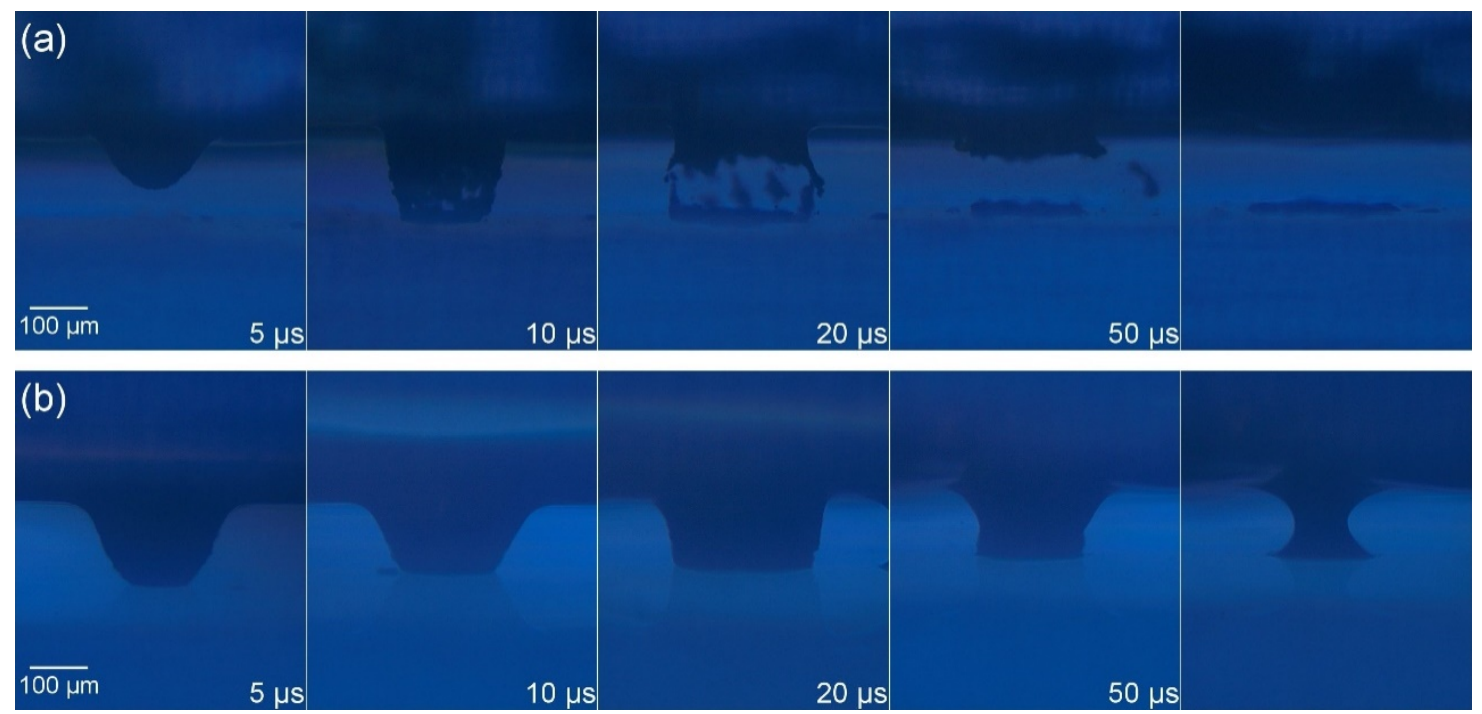

Figure 5.8: Time-resolved images of LIFT of silver paste with a gap of $140 \mu \mathrm{m}$ between donor and acceptor. The film thicknesses and laser fluences are (a) $35 \mu \mathrm{m}$ and $6.8 \mathrm{~J} / \mathrm{cm}^{2}$ and (b) $60 \mu \mathrm{m}$ and $8.4 \mathrm{~J} / \mathrm{cm}^{2}[19]$.

The voxel transfer and imaging results can be understood in terms of the effects of the experimental parameters on the internal dynamics of the paste, similar to previous works on Newtonian fluids [60,66, 99]. As described above, four transfer regimes have been observed, both in the morphology of the transferred voxels and in the time-resolved images: no transfer, cluster-dot transfer, explosive transfer, and concrete transfer. Three important experimental parameters considered here are the laser peak fluence $(F)$, donor thickness $(h)$, and gap distance $(d)$. Fig. 5.9 shows a schematic of the LIFT process according to the results shown above. First, the incident laser pulse is focused onto the glass/paste interface, where it generates a plasma, which rapidly heats and vaporizes the organic component of the silver paste, forming a high-pressure and high-temperature 
vapor bubble [66]. For a given donor thickness $\left(h_{1}\right)$, smaller than $d$, it is possible to define a threshold laser peak fluence $\left(F_{t h}=F_{t h}(h)\right)$ for transfer of material $[17,99]$. The energy threshold of the silver paste is attributable to the internal and external force balance. Using pulse energies below $F_{t h}$, the bubble grows to only a modest size before collapsing, which results in the formation of a blister in the paste (Fig. 5.9a). Above $F_{t h}$, the bubble expands to a larger size due to the larger internal pressure overcoming the ambient air pressure and surface tension force (Fig. 5.9b). The deceleration of the bubble leads to a low-pressure region where the paste protrudes, between the bubble and the paste-air interface, so the silver paste surrounding the bubble flows toward this low-pressure region. The flow and stretching of this protrusion of paste eventually leads to fragmentation into clusters of paste that are transferred to the acceptor substrate. For fluences that are close to $F_{t h}$, most of the kinetic energy in the paste goes into separating these clusters, so that there are relatively few of them and they travel at low velocities (see Fig. 5.5a and Fig. 5.6b). For higher fluences, larger and more violent vapor bubbles are produced, which results in more kinetic energy for fragmentation. The radially outward motion induced by bubble expansion leads to fragmentation into a larger number of smaller pieces that move faster, exploding outwards. The subsequent transfer contains finer droplets and has a larger diameter along with the hole in the donor substrate. The remaining silver paste recoil forms the crustal annular bulge of the crater left on the donor film as seen in Fig. 5.3. 
(a)

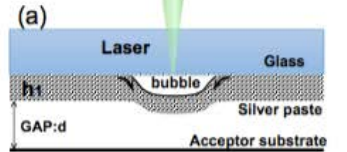

(b)
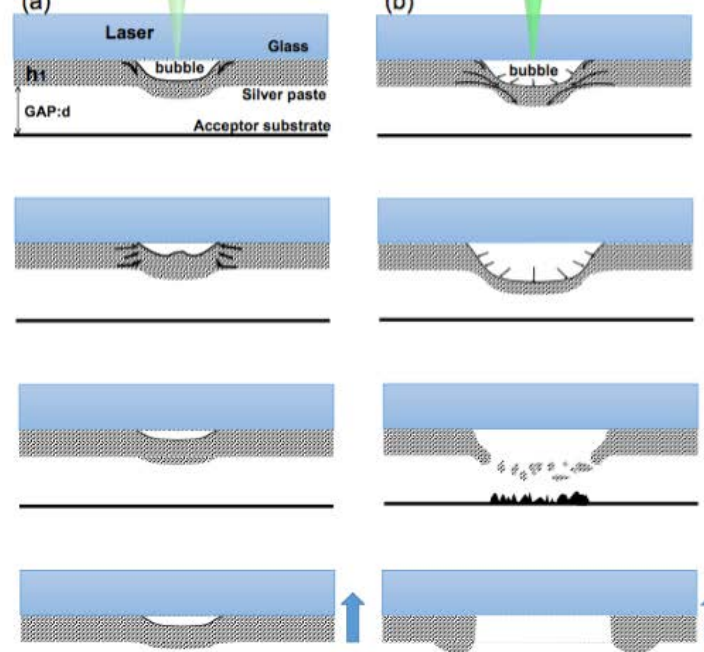

(c)
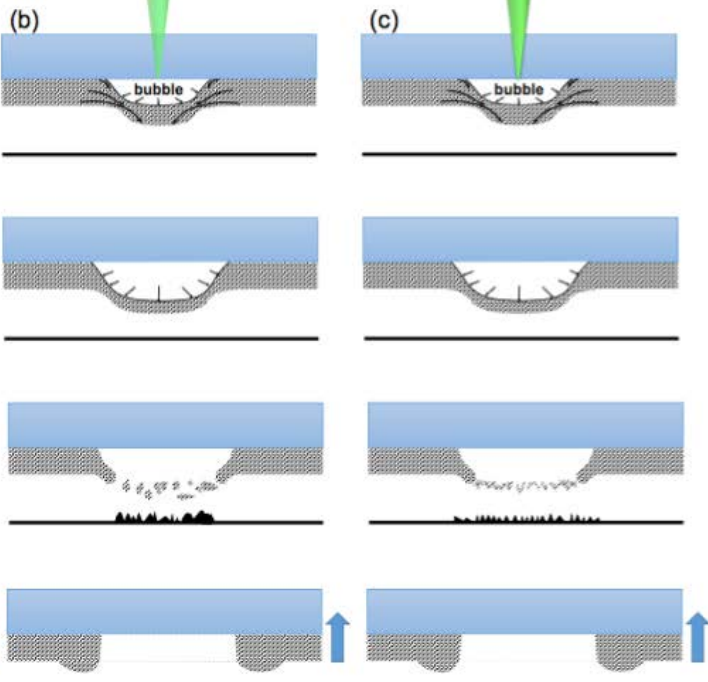
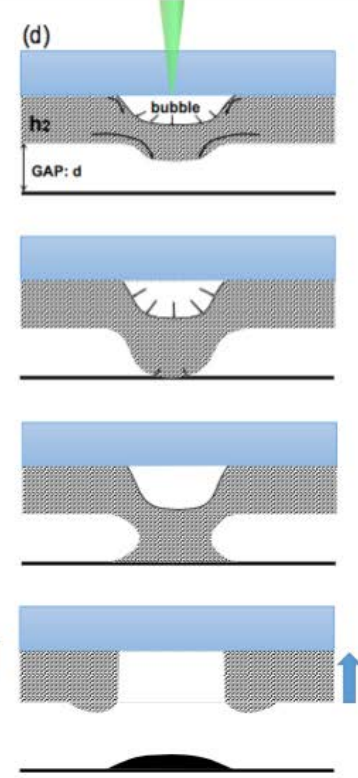

Figure 5.9: Schematic of transfer mechanisms for varying laser peak fluences and donor thicknesses with a fixed gap distance: (a) blister formation, when the fluence is below the transfer threshold; (b) cluster-dot transfer, for fluences slightly above the threshold; (c) explosive transfer, when the peak fluence is much higher than the threshold; and (d) concrete transfer, when the donor is thick enough relative to the gap that the acceptor prevents fragmentation during transfer. The last row shows the final voxel obtained after removing the donor [19].

The progression from being below the threshold for transfer to cluster-dot transfer and eventually explosive transfer as laser fluence increases is similar to that of LIFT with Newtonian liquids, where the most confined and reproducible transfers are obtained just above the threshold and splashing is observed for higher fluences [67]. The distinction between a small number of relatively large clusters at lower fluences and explosions of finer clusters at high fluences (Figs. 5.2a, 5.5 and 5.6) is a new result that arises from the multiphase rheology of the silver paste, however. 


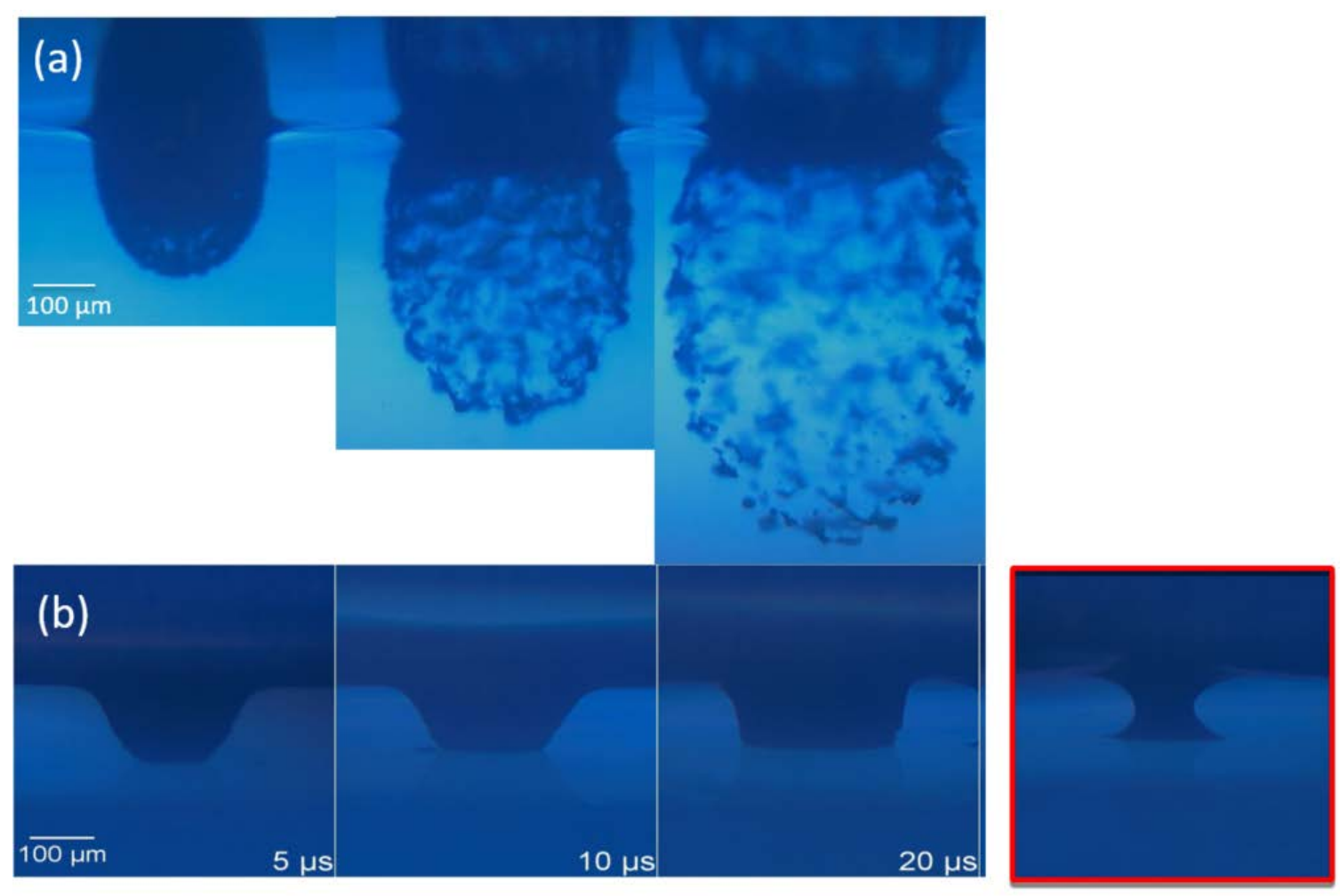

Figure 5.10: Time-resolved images of LIFT of silver paste show the difference between the LIFT in free air and the LIFT with an acceptor substrate with a gap of $140 \mu \mathrm{m}$ between donor and acceptor. The film thicknesses and laser fluences are (a) $35 \mu \mathrm{m}$ and $8.5 \mathrm{~J} / \mathrm{cm}^{2}$ and (b) $60 \mu \mathrm{m}$ and $8.4 \mathrm{~J} / \mathrm{cm}^{2}[19]$.

Fig. 5.10 directly demonstrates that a different transfer mechanism occurs when the donor thickness $h$ is large enough relative to the gap $d$ that the protruding paste pillar reaches the acceptor while connected to the donor substrate. Once the pillar front impacts the acceptor substrate, confinement prevents the paste from fragmenting into pieces, stopping the expansion and stretching of the paste being transferred. Eventually, the laser-induced vapor bubble collapses to an equilibrium shape, leaving a concave pillar taking the form of a liquid bridge wetting both the donor and acceptor. This pillar is stable due to the high viscosity and non-Newtonian character of the paste, inhibiting the spreading and thinning of the impacting pillar and effectively solidifying it. By contrast, in LIFT experiments with Newtonian liquids, jets that impact an acceptor substrate are 
unstable and break up, with some liquid retracting back to the donor film and the rest transferring to a droplet on the acceptor [60].

When the donor substrate is pulled off from the acceptor, the pillar breaks, leaving a single concrete dot of paste on the acceptor substrate. This mechanism explains the lack of splashing in the transfers in Figure $1 \mathrm{~b}$, where the $50-\mu \mathrm{m}$ film is thick enough relative to the 50- $\mu \mathrm{m}$ gap distance so that the pillar impacts the acceptor without breaking up. This effect was suggested by the authors [13] when printing lines with heights larger than the gap distance. Previous LIFT experiments with nanoparticle inks also revealed spikes in the donor film due to remnants of collapsing filaments in the case of nanoparticle inks [15], but this is the first demonstration of stable columns between donor and acceptor substrates.

\subsection{Influence of the paste viscosity}

From the previous experiments, it is known that the thinning of the silver paste can lead to a thin jet. In this section, a thinner is used in order to reduce the viscosity, making the transfer process more liquid-like. A mixture with $4.2 \mathrm{wt} \%$ of the DuPont 9450 thinner is prepared. The voxels are printed by each single laser pulse with varying the pulse energy from 3.2 to $16.5 \mu \mathrm{J}$.
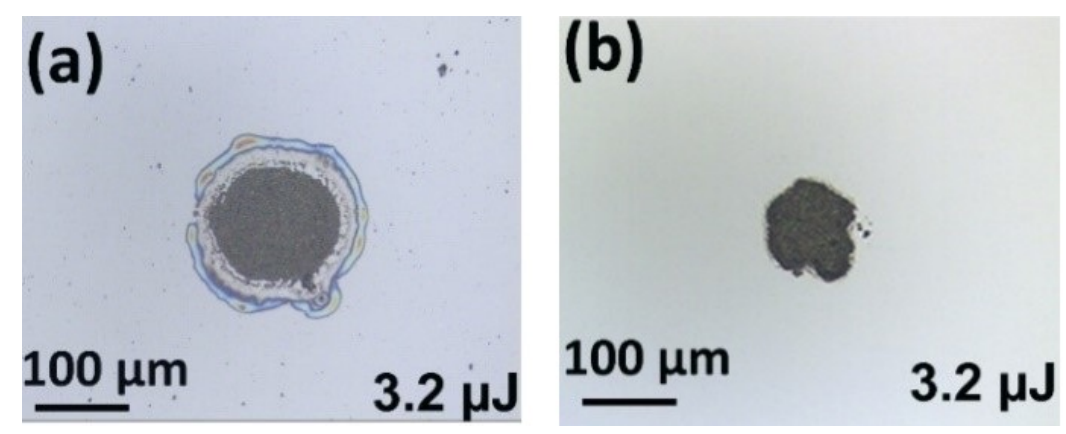

Figure 5.11: Microscope images of LIFT dots transferred using single shots of a ns laser with pulse energy of $3.2 \mu \mathrm{J}$ and donor thicknesses of $50 \mu \mathrm{m}$ (a) With $4.2 \%$ of thinner, (b) original paste. 
Fig. 5.11 represents microscope images of LIFT dots transferred using single shots of a ns laser with pulse energy of $3.2 \mu \mathrm{J}$ and donor thicknesses of $50 \mu \mathrm{m}$ with $4.2 \%$ of thinner and original paste. There is an obvious ring of thinner residue left around the concrete dot. It is also observed tiny paste particles dispersed between the thinner circular and the central concrete part. As a comparison, the voxel with original paste has a smooth edge with only solid component compacted in the center.

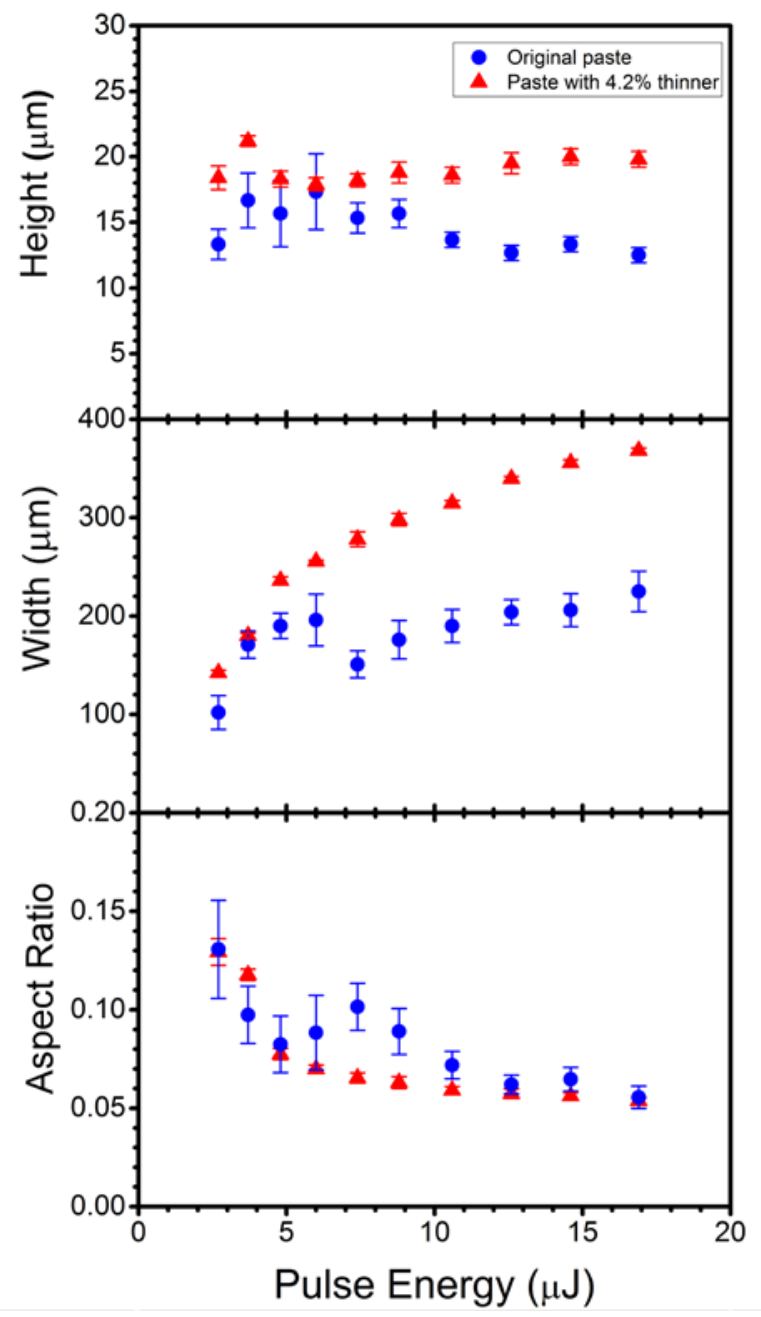

Figure 5.12: The voxel dimension represented by Height, width and aspect ratio of the transferred dots as a function of laser peak fluence with respect to original paste and the paste with $4.2 \%$ with film thickness of $50 \mu \mathrm{m}$. 
From Fig. 5.12, it can be observed that with the $4.2 \%$ of the thinner, both width and height of the voxel are larger than the paste voxel of original paste. However, the aspect ratio of voxel with thinner is larger than the one with original paste. In addition, the transfer threshold energy of the paste with thinner is lower than the original one.

Fig. 5.13 shows the time-resolved images of the paste-thinner mixture for a $60-\mu \mathrm{m}$ thick film and with a pulse energy of $70 \mu \mathrm{J}$ without acceptor substrate. The images clearly illustrate the liquid-like behavior of ejecting collimated jet, similar to those observed with Newtonian fluids. It can be deduced from the behavior observed in the previous sections that when the acceptor substrate is added below, the protruded silver paste will stop disaggregation after several $\mu s$ and start wetting the acceptor surface. However, the thinner reduces the surface energy and yield stress of the original paste, resulting in a longer spreading time on the substrate surface. This finally leads to a larger width of the voxel. Moreover, when thinner is added, the transferred volume should increase since more paste will flow toward the depression area and to the donor so that the height of the voxel is also larger.

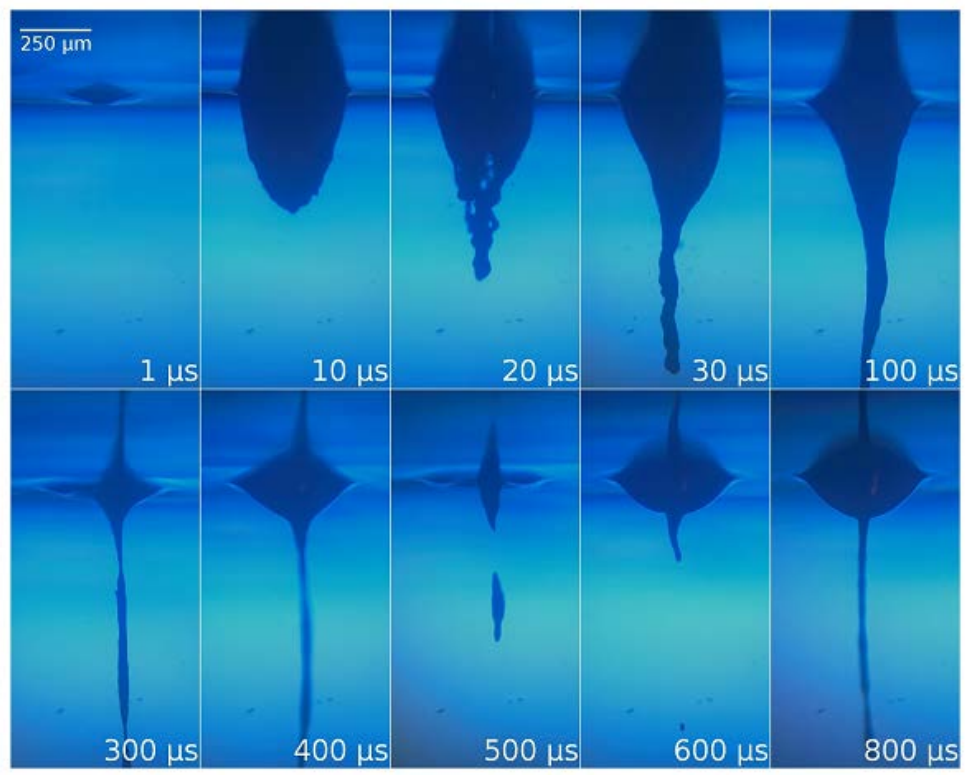

Figure 5.13: Time-resolved images of silver paste with thinner added $(4.2 \%$ by weight). The laser pulse energy is $70 \mu \mathrm{J}$, and the film thickness is about $60 \mu \mathrm{m}$ [104]. 


\subsection{LIFT line results}

At the first beginning the LIFT of line experiments were performed on the surface of texturized c-Si wafer. The lines are deposited by scanning the focused beam using scanner. According to the voxel printing study, it is known that for obtaining a high-aspect ratio voxel transfer the film thickness should be close or even larger than the gap distance in order to prevent splashing of the paste. Thus, for the donor preparation an $80-\mu \mathrm{m}$ thick film and $50-\mu m$ gap distance is chosen for the LIFT line printing. The laser repetition rate is fixed at $20 \mathrm{kHz}$. The variable parameters are the pulse energy and scan speed. After a process parameterization of the line, it is found that the optimal process parameters with laser pulse energies around $20 \mu \mathrm{J}$ and large speeds $(2 \mathrm{~m} / \mathrm{s})$ in the LIFT line printing. Then the pulse energy is slightly varied for finding the best result.

Fig. 5.14 shows the photograph of LIFT line with $18 \mu \mathrm{J}$ of pulse energy. It can be seen that although the line can be transferred to acceptor substrate most parts of the line are discontinuous. Fig. 5.15 illustrates the confocal images of these LIFT lines. The width of the line varies from 85 to $120 \mu \mathrm{m}$ which means the transferred parts of the line is not homogenous. This defect can be caused by the non-uniformity of the silver paste during the coating procedure or the non-uniformity of the mechanical force that acting on the donor when lifting the donor substrate manually.
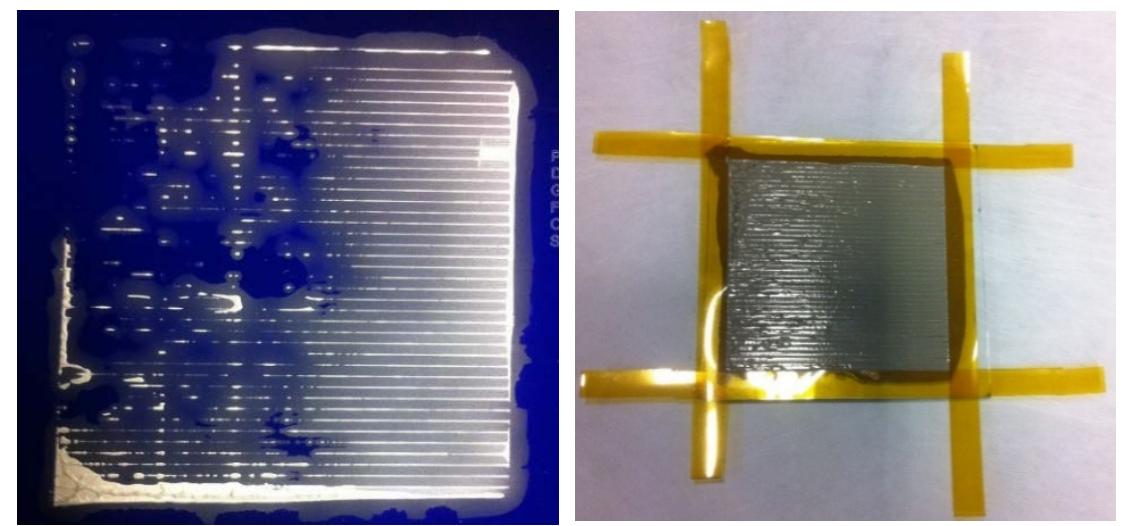

Figure 5.14: LIFT lines of silver paste on a texturized c-Si wafer and its donor substrate. obtained with these process parameters: Paste thickness: $80 \mu \mathrm{m}$; Gap: $50 \mu \mathrm{m}$; pulse energy: $18 \mu$; Frequency: 20 kHz; Speed: $2.0 \mathrm{~m} / \mathrm{s}$. 

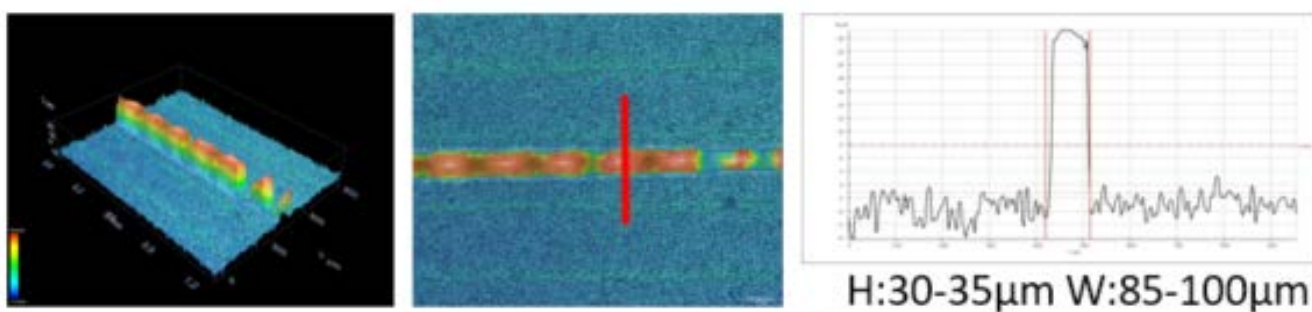

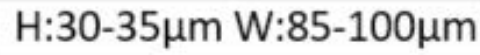
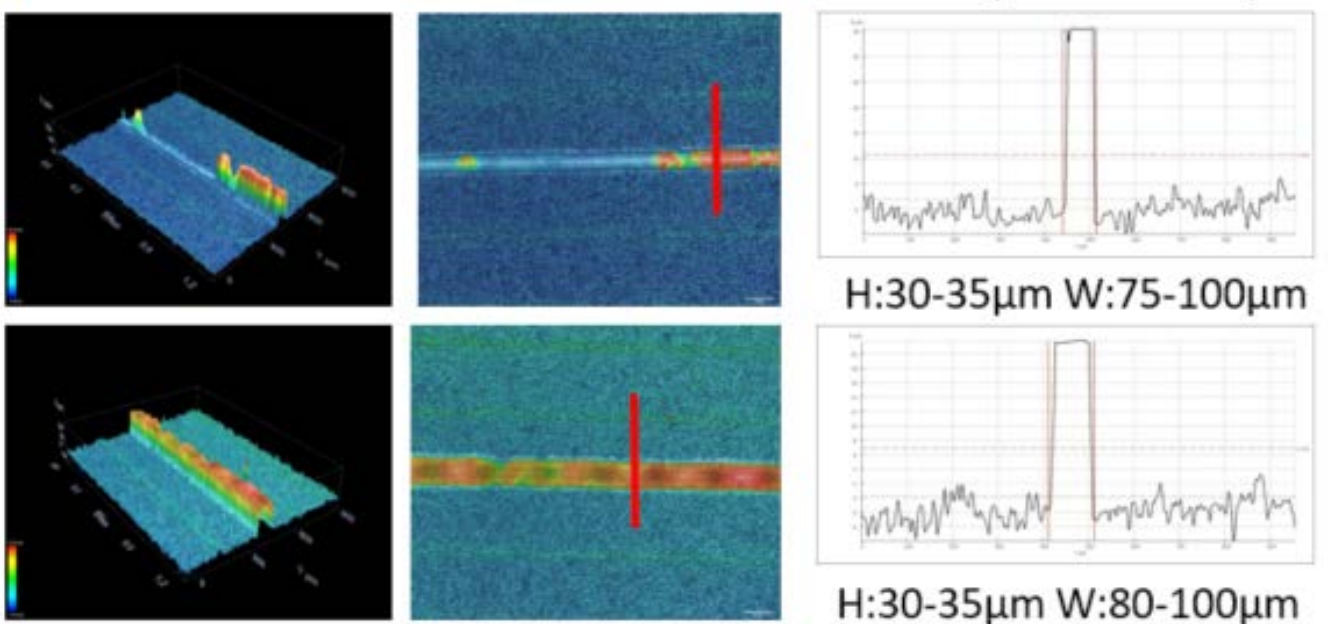

$\mathrm{H}: 30-35 \mu \mathrm{m}$ W:75-100 $\mathrm{mm}$
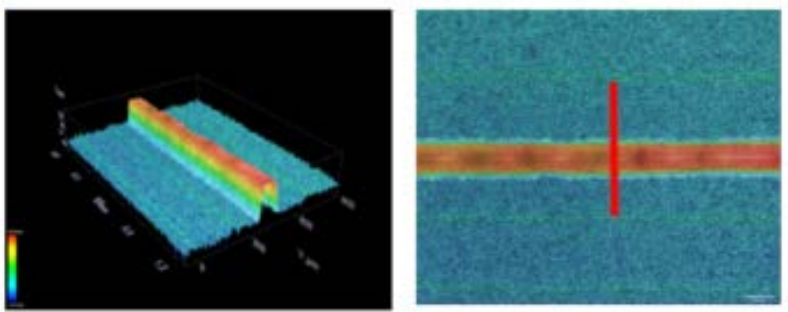

$\mathrm{H}: 30-35 \mu \mathrm{m}$ W:80-100 $\mu \mathrm{m}$

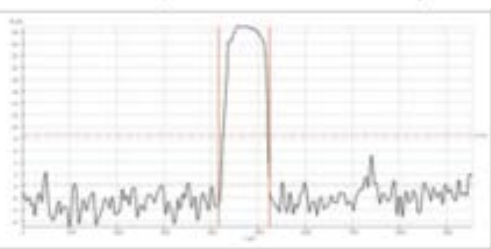

$\mathrm{H}: 30-35 \mu \mathrm{m}$ W:100-120 $\mu \mathrm{m}$

Figure 5.15: Confocal images of the LIFT lines obtained with these process parameters: Paste thickness: $80 \mu \mathrm{m}$; Gap: $50 \mu \mathrm{m}$; pulse energy: $18 \mu$ j; Frequency: $20 \mathrm{kHz}$; Speed: 2.0 $\mathrm{m} / \mathrm{s}[13]$

Fig. 5.16 shows the photograph of LIFT line with $20 \mu \mathrm{J}$ of pulse energy. It can be seen that the line can be transferred to acceptor substrate most parts of the line are continuous. Fig. 5.17 illustrates the confocal images of these LIFT lines. The width of the line varies from 80 to $140 \mu \mathrm{m}$ which means the transferred parts of the line is not homogenous. The line is formed by overlapping adjacent two transferred voxels together. The part of line with largest height (peak) should be the center of the printed voxel and 
in the connecting point between two peaks the height is the lowest (valley). The largest height in the line is $65 \mu \mathrm{m}$ and the lowest height is $40 \mu \mathrm{m}$.
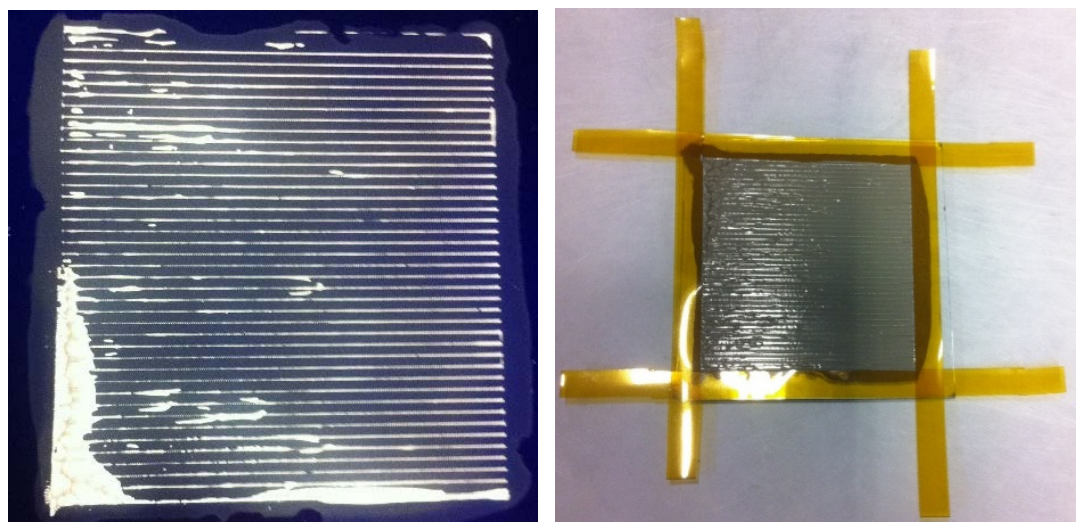

Figure 5.16: LIFT lines of silver paste on a texturized c-Si wafer obtained with these process parameters: Paste thickness: $80 \mu \mathrm{m}$; Gap: $50 \mu \mathrm{m}$; pulse energy: $18 \mu \mathrm{J}$; Frequency: $20 \mathrm{kHz}$; Speed: $2.0 \mathrm{~m} / \mathrm{s}$.
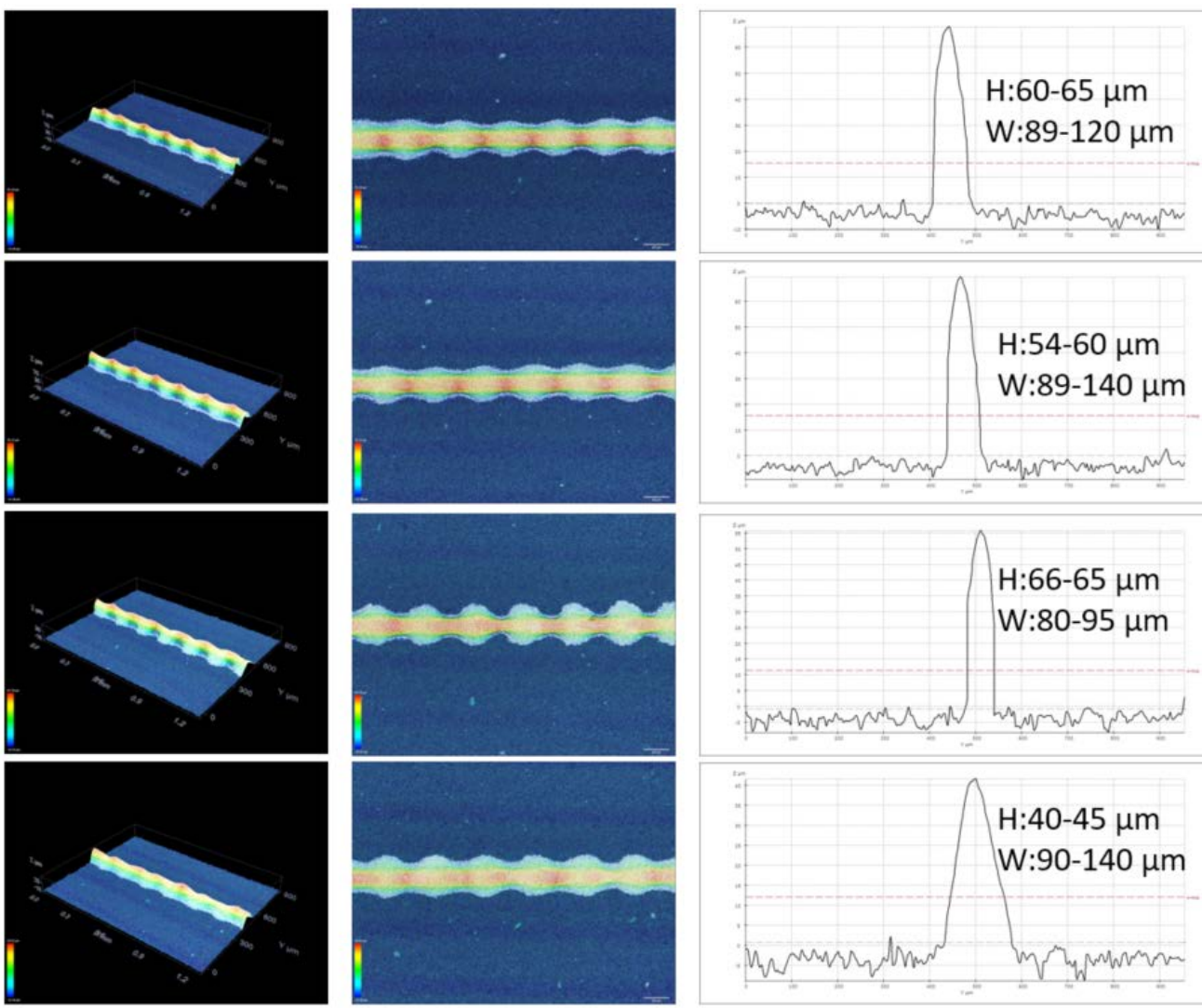

Figure 5.17: Confocal images of the LIFT lines obtained with these process parameters: Paste thickness: $80 \mu \mathrm{m}$; Gap: $50 \mu \mathrm{m}$; pulse energy: $18 \mu \mathrm{J}$; Frequency: $20 \mathrm{kHz}$; Speed: 2.0 $\mathrm{m} / \mathrm{s}[13]$. 

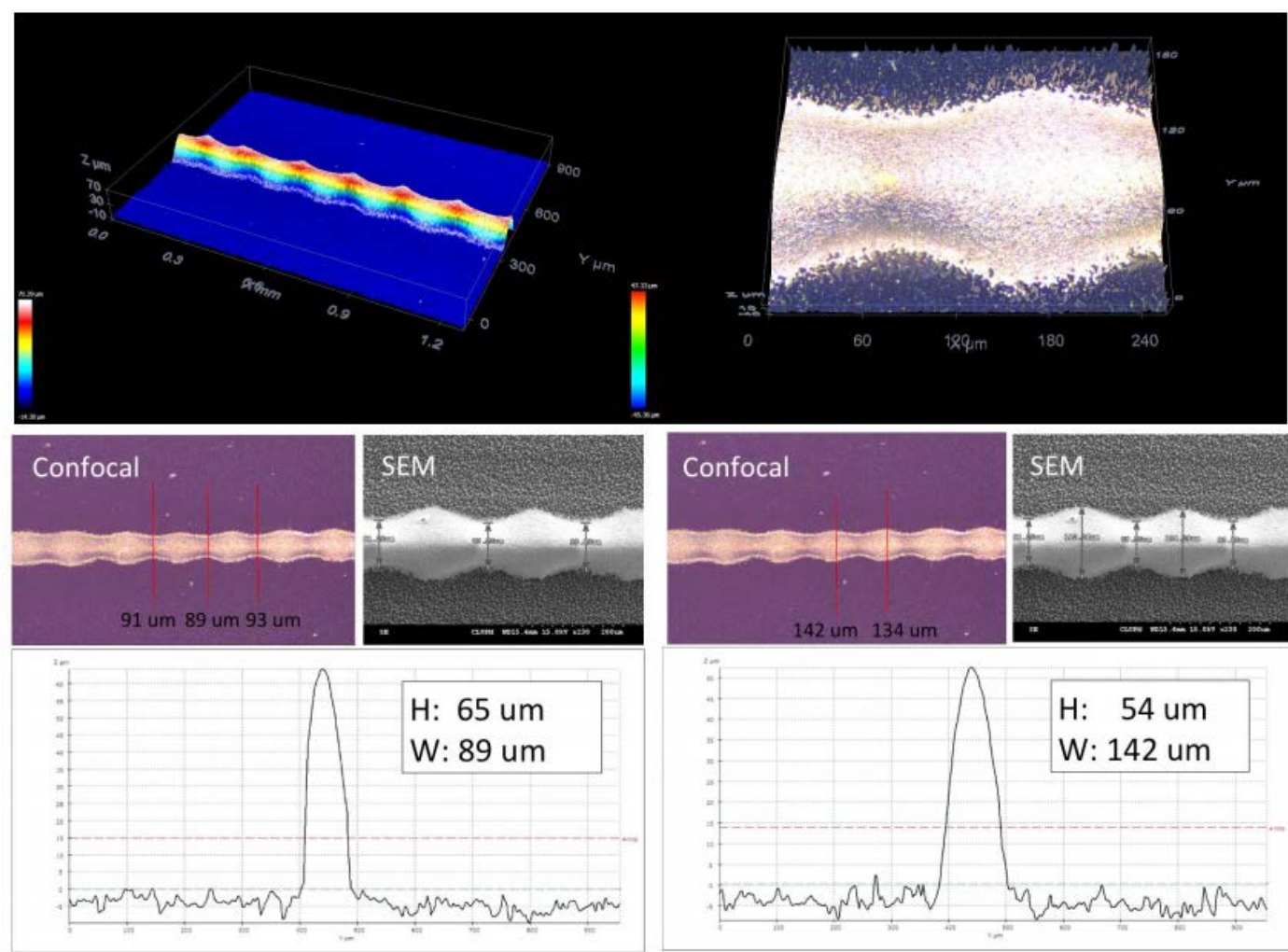

Figure 5.18: Different LIFT lines confocal and SEM profiles obtained with these process parameters: Paste thickness: $80 \mu \mathrm{m}$; Gap: $50 \mu \mathrm{m}$; pulse energy: $20 \mu \mathrm{J}$; Frequency: $20 \mathrm{kHz}$; Speed: $2.0 \mathrm{~m} / \mathrm{s}[13]$.

The largest height in the line is $65 \mu \mathrm{m}$ and the lowest height is $40 \mu \mathrm{m}$. Thus, it is possible to transfer lines with high aspect ratios: 0.36 - 0.61 (width: 90-150 $\mu \mathrm{m}$, height: $55 \mu \mathrm{m}$ ) and without discontinuities (Fig. 5.18 and Fig. 5.19). This approach is able to transfer large volume voxels, larger than $300 \mathrm{pL}$. The mechanism of this transfer process is discussed in the previous section. One of the advantages of LIFT technique over the traditional screen printing for silver paste finger line printing is the ability of obtaining large height line (more than $30 \mu \mathrm{m}$ ). This result proved the feasibility of LIFT process for printing of finger line with high aspect ratio in an industrial process speed on solar cell. 

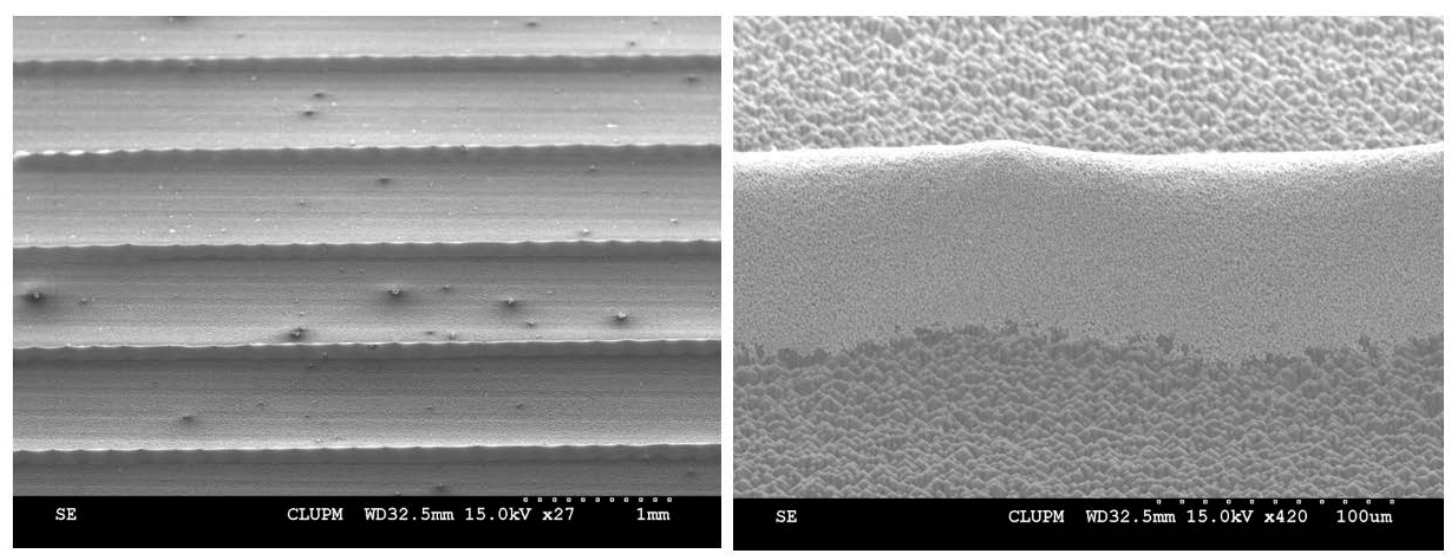

Figure 5.19: SEM images of LIFT lines confocal obtained the optimal process parameters: Paste thickness: $80 \mu \mathrm{m}$; Gap: $50 \mu \mathrm{m}$; pulse energy:20 $\mu$ J; Frequency: 20 kHz; Speed: 2.0 $\mathrm{m} / \mathrm{s}$.

\subsection{Influence of the wetting with different acceptor in LIFT}

It has also been found that if the acceptor substrate is changed the wettability is changed and the morphology of the voxel or line is also changed. This is especially clear when LIFT is done using as acceptor a CIGS solar cell; with the same processing parameters, the line width is always wider and the aspect ratio is smaller than the case of texturized c-Si.

\begin{tabular}{|c|c|}
\hline Processing Parameters & Values \\
\hline Laser frequency & $20 \mathrm{KHz}$ \\
\hline Laser spot size & $20 \mu \mathrm{m}$ \\
\hline Pulse duration & $15 \mathrm{~ns}$ \\
\hline Paste & DuPont 17F+thinner 9450(4.2 wt\%) \\
\hline Paste thickness & $50 \pm 5 \mu \mathrm{m}$ \\
\hline GAP & $50 \mu \mathrm{m}$ \\
\hline Receiving substrate & Polished c-Si, textured c-Si, CIGS \\
\hline Focus position & On focus \\
\hline
\end{tabular}

Table 5.1: LIFT processing parameters of voxel printing on different substrate. 
Clearly, the substrate produces an effect on the morphology of the printed voxel. Therefore, voxel printing experiments on three different substrates (textured c-Si, polished c-Si, CIGS with TCO film surface) were performed using same LIFT process parameter which is shown in Table. 5.1.

Voxels and lines are printed onto three different substrates (textured c-Si, polished c-Si and CIGS with a TCO film on top) using LIFT technique. The LIFT experimental set-up is described elsewhere [19]. As donor film a commercial micro-sized silver paste (DuPont Solamet PV17F) is used. In order to avoid the degradation of paste by aging and reduce the paste viscosity, a $4.2 \mathrm{wt} \%$ of thinner (DuPont 9450) is added to the paste. The final viscosity is $54 \pm 3 \mathrm{~Pa} \cdot \mathrm{s}$ (measured as described in reference 3 ). The thickness of the donor layer was $50 \pm 10 \mu \mathrm{m}$. The gap distance between donor and acceptor (50 $\mu \mathrm{m})$ was controlled by sticking Kapton tapes. Voxels are printed at different pulses energies, from 2.7 to $16.9 \mu \mathrm{J}$ (peak fluences $F_{0}$ from 1.1 to $6.9 \mathrm{~J} / \mathrm{cm}^{2}$ ). Lines are printed with a pulse energy of $2.7 \mu \mathrm{J}\left(F_{0}=1.1 \mathrm{~J} / \mathrm{cm}^{2}\right)$ and scan speed of $1.8 \mathrm{~m} / \mathrm{s}$. The morphology of voxels and lines was characterized using confocal microscopy and SEM.

Fig. 5.20 shows the voxel morphology (height, width and aspect ratio) as a function of the laser pulse energy for the different acceptors. Each data point in the figure represents the average of 5 measurements, with error bars denoting one standard deviation. As it can be seen in the figure, the voxel width on the polished c-Si is the largest among the three, while on the textured c-Si substrate the voxel width has its minimum value. In both three cases, the width of the voxels increases as a function of pulse energy, while the aspect ratio with pulse energy shows a decreasing trend. Therefore, the minimum pulse energy $(2.7 \mu \mathrm{J})$ for obtaining the voxel with the largest aspect ratio is selected for printing lines. Aspect ratio of the lines on polished c-Si has the minimum value of 0.10 , while on textured c-Si substrate the aspect ratio is 0.3 , and on CIGS substrate it has an intermediate value of 0.18 (see Fig. 5.21-23). 
In principle, the transferred voxel volume, i.e. the amount of paste pushed from the donor, should be same when using the same pulse energy. However, experimental results show that there are strong differences depending on the nature of the substrate. Those differences could be related to the wetting properties of the substrates. In order to investigate this effect, the surface morphologies of the acceptors are examined by Confocal microscopy. The mean roughness $R_{a}$ of the three substrates are $90 \pm 30 \mathrm{~nm}$ (polished c-Si), $490 \pm 30 \mathrm{~nm}$ (CIGS) and 1040 $\pm 30 \mathrm{~nm}$ (textured c-Si).

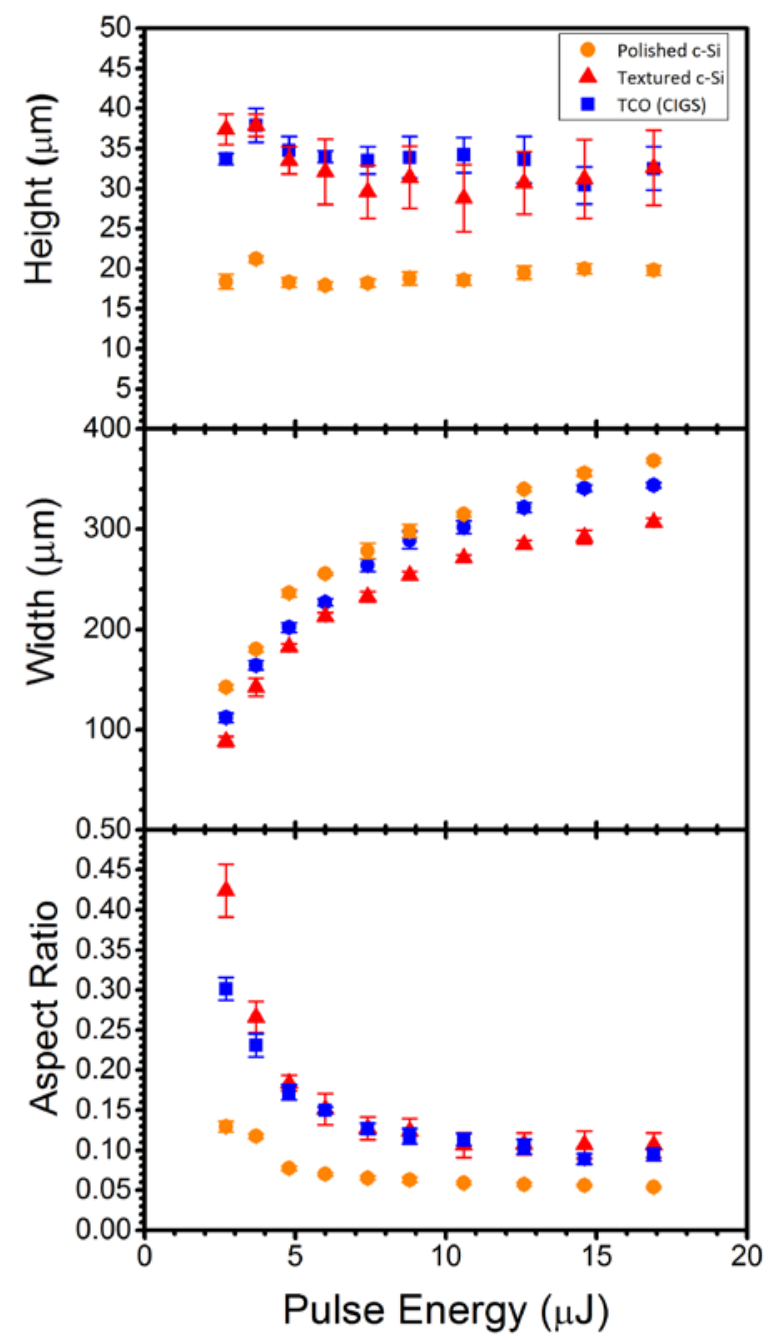

Figure 5.20: Height, width and aspect ratio of the transferred dots with different substrate (Polished c-Si, Textured c-Si and TCO film of CIGS) as a function of laser peak fluence with respect to two different paste thicknesses ( $30 \mu \mathrm{m}$ and $50 \mu \mathrm{m})$. 
Fig. 5.24 shows SEM images of the cross-section of lines printed using a pulse energy of $2.7 \mu \mathrm{J}$ onto the three substrates. The contact angles $\left(\theta_{c}\right)$ between the paste and the surface are $20^{\circ}$ (polished c-Si), $30^{\circ}$ (TCO film on CIGS), and $75^{\circ}$ (textured c-Si). The differences on the roughness of surface are also illustrated: polished c-Si and CIGS substrate with TCO layer have a flatter surface than the textured c-Si substrate, the latter having a small pyramid pattern in the order of microns.

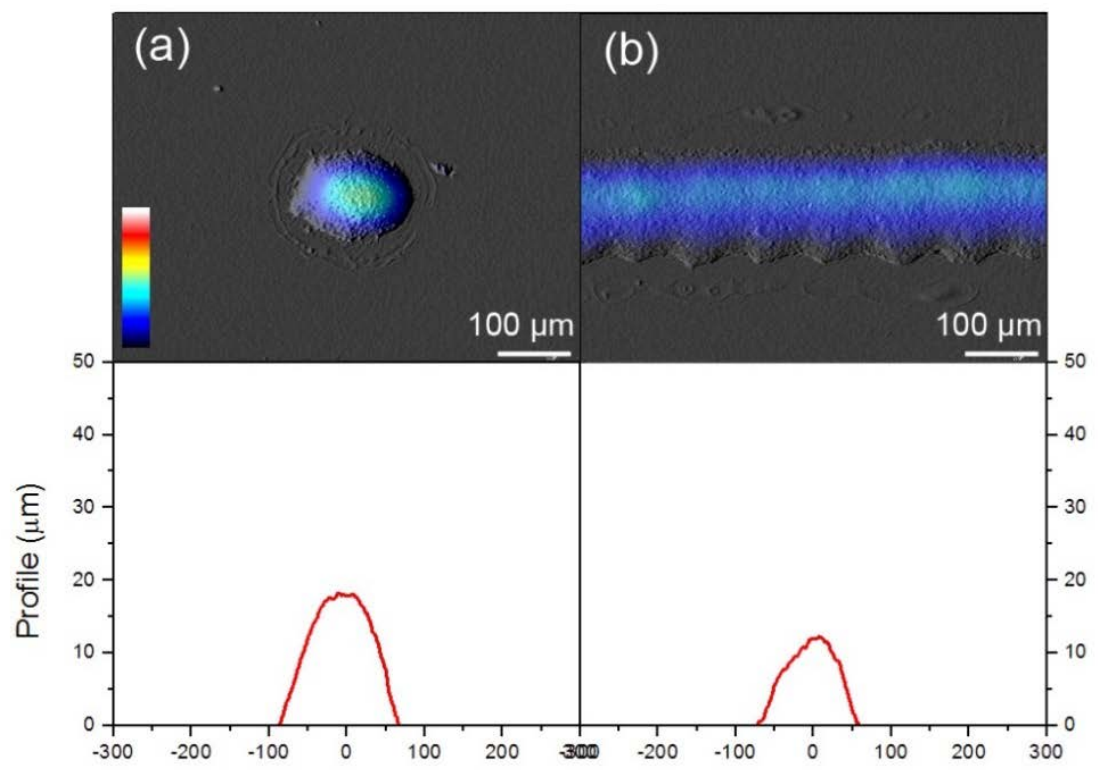

Figure 5.21: Confocal images of the voxel and line printed on polished c-Si substrate with laser pulse energy of $2.7 \mu \mathrm{J}$ : (a) voxel, (b) line.

Before it has been known that the concrete-dot transfer of high viscosity micronsized pastes involves a process in which the transferred material touches the acceptor and forms a stable bridge between donor and acceptor substrate [19]. The force of adhesion is enhanced by filling the silver paste into the pattern of substrates as a wetting process, which is favorable for bonding each other with a high mechanical interlocking that increases the contact area between the fluid and solid surface. That favors the formation of the stable bridge between donor and acceptor, and thus concrete-dot transfer of single voxels without debris. Moreover, the pyramid patterns affect the mean 
contact angle of the voxel by impeding the silver paste from spreading along the surface. Both effects lead to a larger aspect ratio of line when compared to the smother substrates.

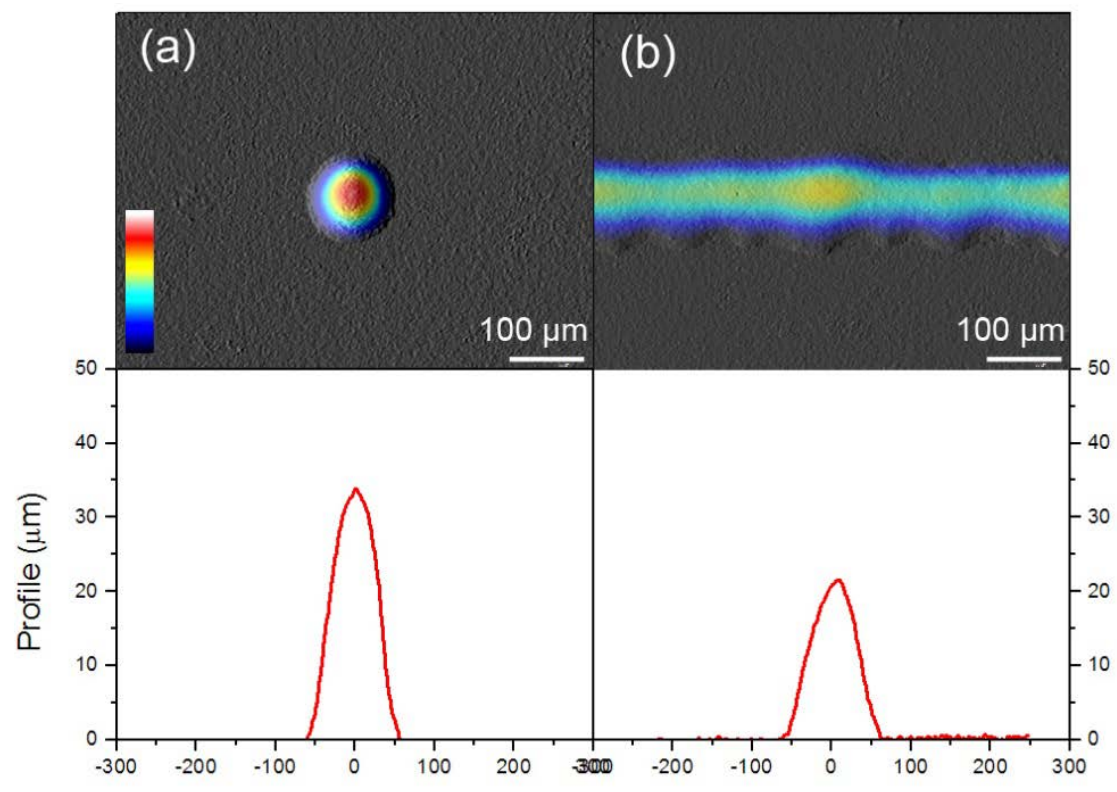

Figure 5.22: Confocal images of the voxel and line printed on CIGS substrate with laser pulse energy of $2.7 \mu \mathrm{J}$ : (a) voxel, (b) line.

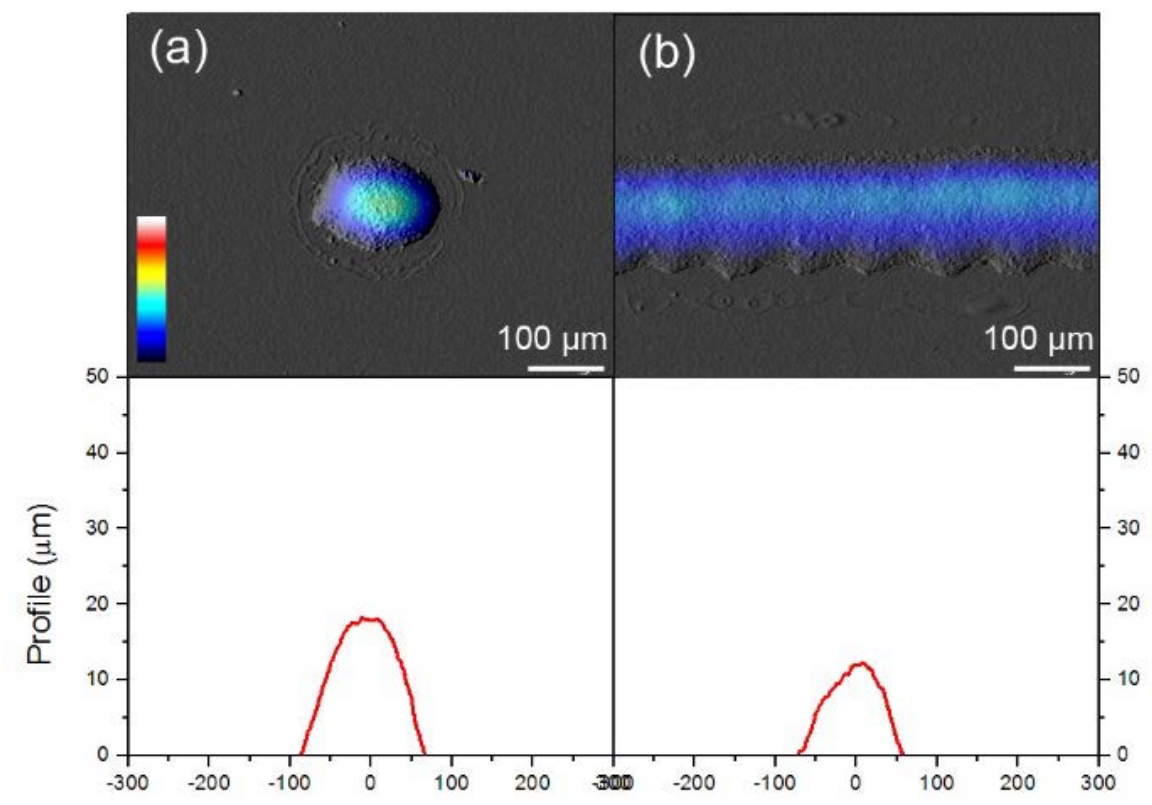

Figure 5.23: Confocal images of the voxel and line printed on textured c-Si substrate with laser pulse energy of $2.7 \mu \mathrm{J}$ : (a) voxel, (b) line. 

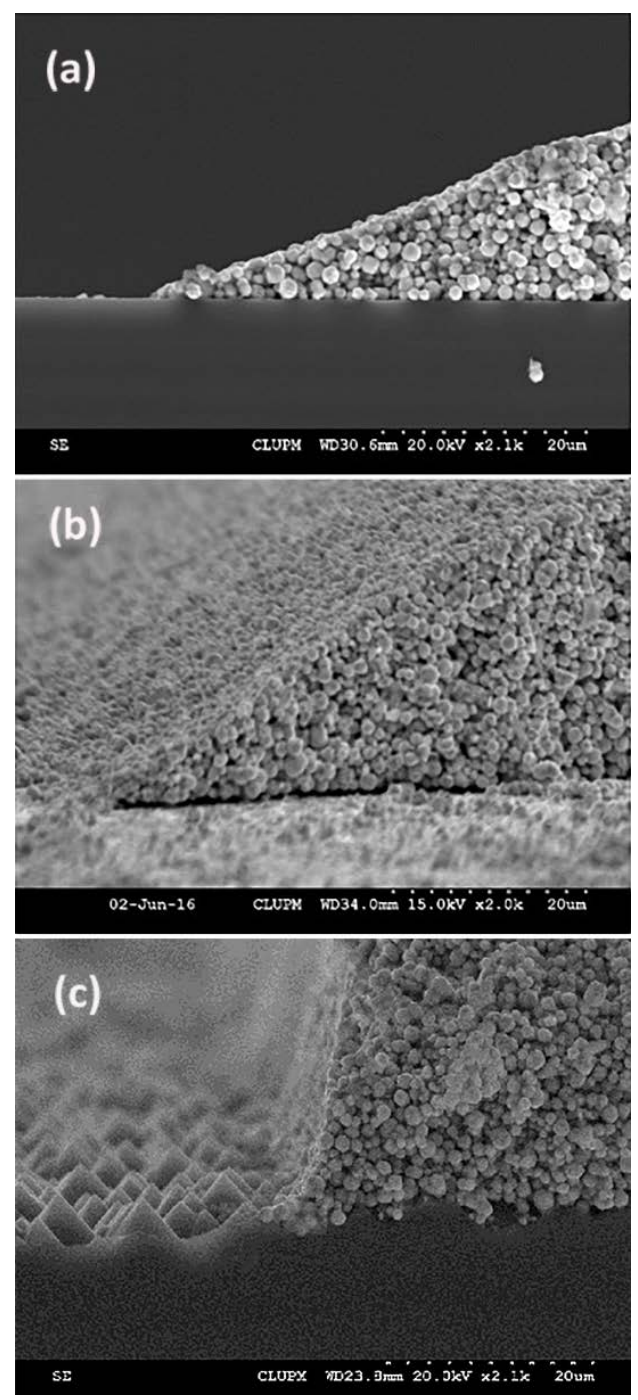

Figure 5.24: SEM micrographs of cross-section of lines printed using the same experimental conditions onto different substrates: (a) polished c-Si (b) TCO film on CIGS, (c) textured c-Si. The contact angles between the paste and the substrate surface are respectively $20^{\circ}, 30^{\circ}$, and $75^{\circ}$.

It can be concluded that in order to increase the adhesion force, the liquid should highly wet the surface of the substrate. Increasing the surface roughness by texturing is an efficient method for solving this problem. When using a highly textured surface, the increased contact area provides a high adhesion force that prevents the paste from 
spreading along the surface, which shapes the voxel with a smaller width and larger height, i.e. an enhanced aspect ratio.

\subsection{Large area and design flexibility of LIFT process}

The use of renewable energy into architectural design is a field of the greatest importance to fulfill the present and future requirements of reduction of fossil fuels consumption. Under this scenario, an undeniable opportunity of developing and optimizing existing low carbon technologies emerges. Until now, PV technologies have been focused in the development of solar farms that do not take all the advantages and possibilities of this renewable source, and in most cases has been treated as a financial product due to the high incentives. Currently, big energy markets like Europe or USA require other business models. Nowadays, in the case of PV, the efforts should be focused in on-site energy generation as DER (distributed energy resource). In other words to bring the energy where it is demanded: the buildings. In this sense, the first approach has been carried out by means of BAPV (Building Applied Photovoltaics) leading critical main limitations: a) it requires an additional investment within the building budget, b) aesthetical does not fulfil the requirements of most of the building stakeholders showing poor acceptability and c) does not comply with key building codes limiting its application. Therefore, it is easy to understand that BIPV has the chance to become the most attractive market for PV technologies. This is based on the capacity of providing multifunctional solutions to buildings throughout active and passive properties allowing on-site RES (Renewable Energy Sources) energy production, thermal envelope benefits and daylighting entrance at the same time. At present, BIPV systems still represent a small share of the PV market mainly due to the mistaken belief that BIPV integrations are more expensive than conventional roof installations and that the strong incentives given in the past were associated only with "utility" products and not-distributed generation. Under this scenario, it is mandatory to carry out an effort in developing and breaking into the market of multifunctional BIPV solutions capable to satisfy the demands of main stakeholders in 
building sector in a holistic manner (cost, aesthetic, technical and structural needs) taking into account the benefits associated to the building users. In this context laser technology appears as key enable technology to fulfill two of the cited requirements: cost and aesthetic.

In fact lasers have been used in microelectronic and photovoltaic (PV) industry for decades. More recently, laser technology has appeared as a key enabling technology to improve efficiency and to reduce production costs. This tool is present not only in standard c-Si PV factories but it is becoming a fundamental technology to develop new concepts both in HE (High Efficiency) solar cells fabrication and in most of the TF (Thin film) photovoltaic technologies. In addition, laser technology, especially LIFT technology, which can be used to print freeform front grids without the need of any screen, could be a fundamental tool to solve a key problem in BIPV: the customization of standard PV devices to fulfil the cost and aesthetic criteria imposed by the architectural design [105].

Figure 5.25 shows the possibilities of this technique, with an $18 \mathrm{~cm}^{2}$ hexagonal pattern cell design. This allows the printing of different grids just changing the program of the scanner or the motorized stage, giving a great flexibility to the metallization process. Moreover, we proved that LIFT could be made in large areas while most research is done in small areas with a bigger focus on precision. And LIFT is a very versatile printing technique, allowing any freeform design for the solar cells personalization (see Fig. 5.26). 


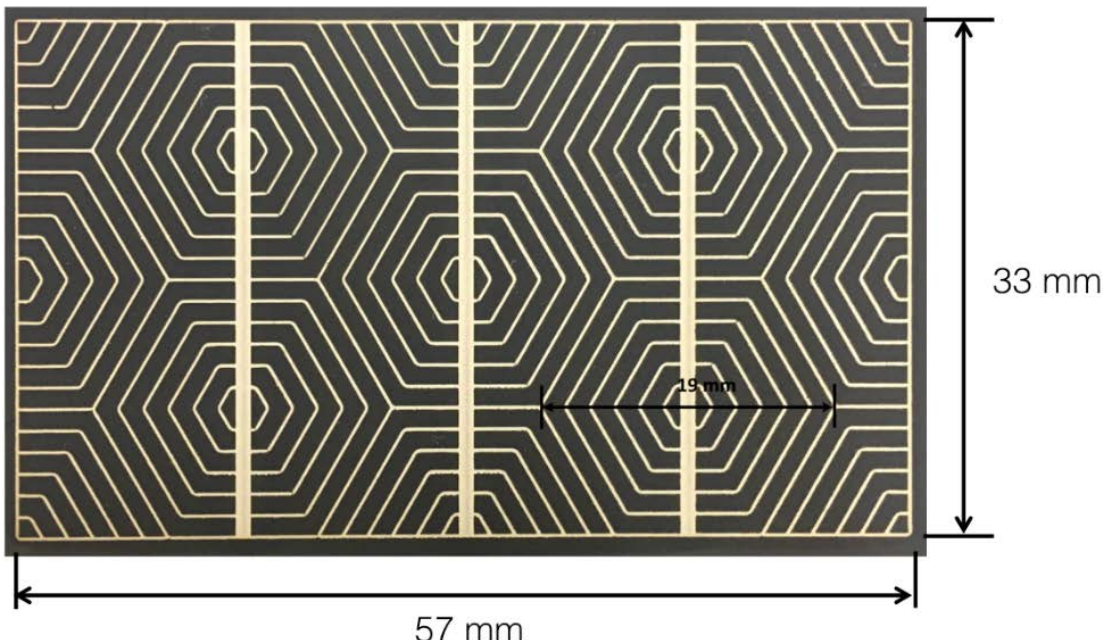

Figure 5.25: Front grid printed with LIFT on a c-Si cell.
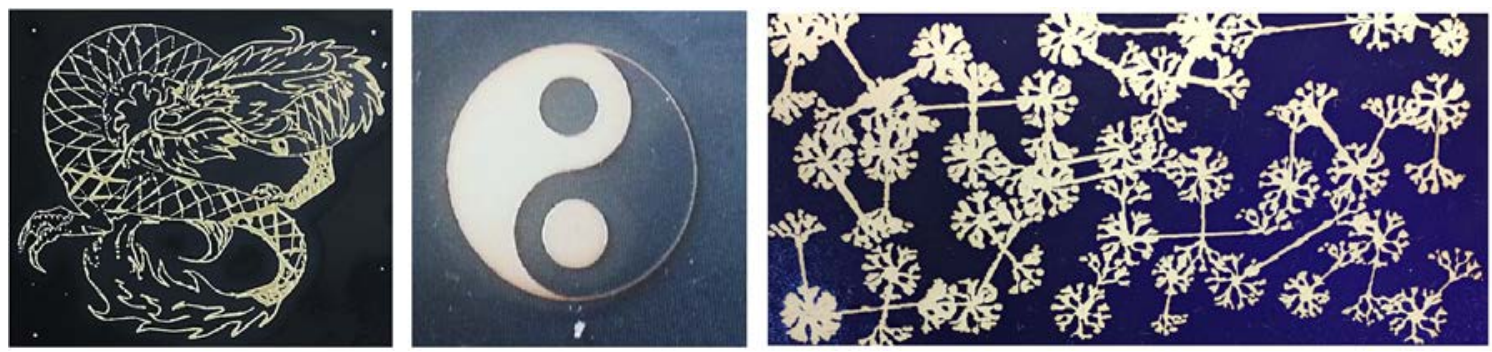

Figure 5.26: Some freeform patterns printed with LIFT on texture c-Si cell. 


\section{Picosecond LIFT results}

Two kind of ps laser were employed in the present LIFT study. One is a high power DPSS ps-laser (Ekspla Atlantic 355-60), emitting in the $532 \mathrm{~nm}$ harmonic and with pulse duration of 13 ps. The other is Onefive, Katana-10 HP (serial number 826), emitting in $1064 \mathrm{~nm}$ wavelength. The optical path comprises a $5 \times$ beam expander (Sill Optics) and a fixed focusing lens (Linos Focus-Ronar) with focal length of $58 \mathrm{~mm}$. Laser beam is focused (beam waist: $\sim 10 \mu \mathrm{m}$ ) onto the interface between the transparent donor substrate and the film of the material to be transferred. A computer-controlled $X-Y$ linear translation stage allows displacing both donor and acceptor substrates respect to the laser beam.

In the LIFT experiment the same commercial silver paste (DuPont Solamet PV17F) was applied as the donor material. The preparation is the same as discussed in the Chapter 3. The paste thickness was $35 \pm 10 \mu \mathrm{m}$ and it was measured in each donor sample before LIFT experiments in the border of the film, and after LIFT experiments in the hole left on the film by the laser pulse. A c-Si wafer is used as acceptor substrate. Donor and acceptor substrate where separated at a controlled distance by means of sticking Kapton tapes onto the donor substrate. The morphology of the transferred paste and the holes left on the donor substrate after LIFT process has been characterized using a confocal microscope (Leica DCM3D).

Initially, we studied the transfer of single dots of paste or voxels using single laser pulses. The main experimental parameters in the LIFT process are the laser fluence, the film thickness and the gap distance. In the present work, the film thickness is fixed $(35 \pm 10$ $\mu \mathrm{m})$ and two different gap distance have been studied (50 and $100 \mu \mathrm{m}$ ) in a wide range of laser pulse energy, from 1.7 to $57.3 \mu \mathrm{J}$. 


\subsection{Printing of single voxel}

\subsubsection{Transfer threshold energy}

In the second experiment, we use different range of pulse energy to find out the transfer threshold energy and to investigate the influence of pulse energy with varying attenuation value from $20 \%$ to $100 \%$, with respect to laser pulse energy from $8.3 \mu \mathrm{J}$ to $57.3 \mu \mathrm{J}$ (see Fig. 6.1). The GAP distance in this experiment is $100 \mu \mathrm{m}$.

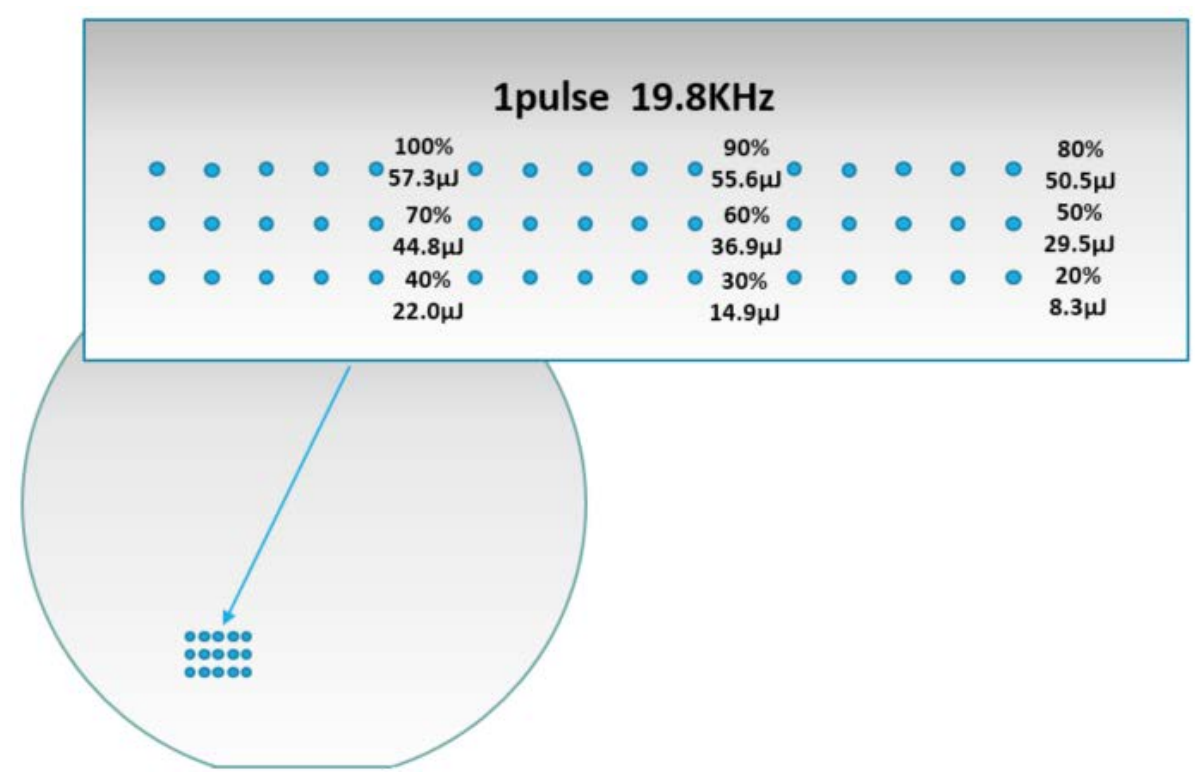

Figure 6.1: The schematic of voxel printing with ps-pulsed laser. The laser pulse energy from $8.3 \mu \mathrm{J}$ to $57.3 \mu \mathrm{J}$ (a) width of the hole, (b) depth of the hole, (c) width of the voxel, (d) height of the voxel.

The results demonstrate that both widths of the hole and the voxel increase as a function of the laser pulse energy (Fig. 6.2), which is in accord with the results of the nanosecond LIFT experiment. The depth of the hole in the donor keeps almost the same value, which is equal to the film thickness. It indicates that within this pulse energy ranges the laser cleans off the hole. With $20 \%$ of the attenuation of the laser pulse energy $(8.3$ $\mu J)$ the silver paste voxel can be transferred. Therefore, the transfer threshold energy should be around this value. 

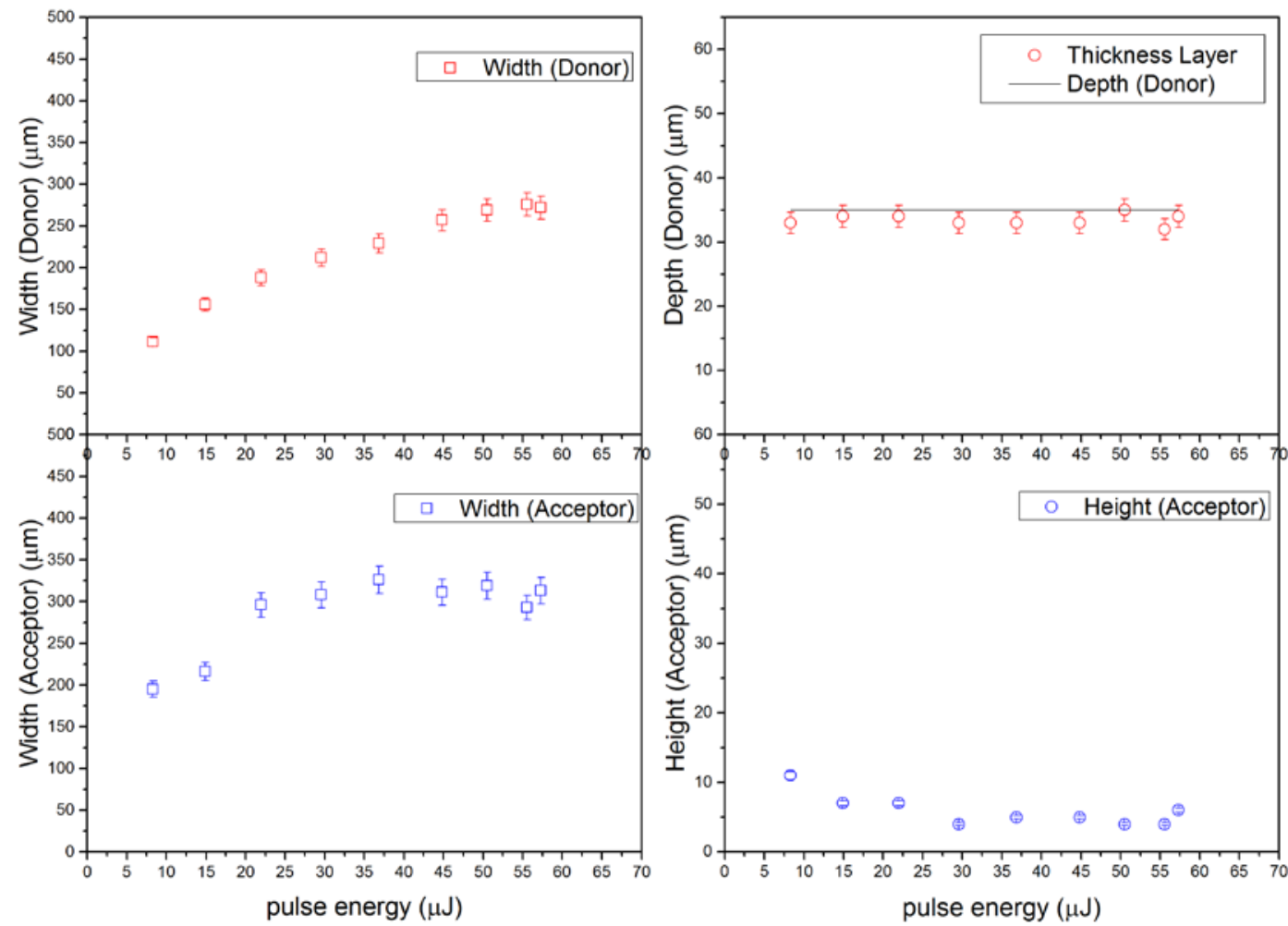

Figure 6.2: The dimension of the hole left in the donor and the voxel deposited on the acceptor as a function of laser pulse energy from $8.3 \mu \mathrm{J}$ to $57.3 \mu \mathrm{J}(\mathrm{a})$ width of the hole, (b) depth of the hole, (c) width of the voxel, (d) height of the voxel.

Then pulse energy was varied again by adjusting different attenuation value from $20 \%$ $(8.3 \mu \mathrm{J})$ to $4 \%(0.7 \mu \mathrm{J})$ with reducing $2 \%$ of each step to find out the precise threshold value since this threshold value will be used to make multiple pulse LIFT experiment.

The results show that below $18 \%$ of the attenuation value $(6.9 \mu \mathrm{J})$ there is nothing printed on the acceptor substrate (see Table 6.1). Therefore, the transfer threshold energy value in this case should be around $6.9 \mu \mathrm{J}$. From the donor, it is observed that the widths of the hole minimize as a function of the laser pulse energy while the depth of the hole increase. 


\begin{tabular}{lllll}
\hline & Acceptor (Voxel) & & \multicolumn{2}{c}{$\begin{array}{l}\text { Donor } \\
\text { (hole) }\end{array}$} \\
\hline Attenuation(\%) & Width $(\mu \mathrm{m})$ & Height $(\mu \mathrm{m})$ & Width $(\mu \mathrm{m})$ & Depth $(\boldsymbol{\mu m})$ \\
20 & little printed & little printed & 100 & 41 \\
18 & little printed & little printed & 73 & 41 \\
16 & nothing printed & nothing printed & 101 & 14 \\
14 & nothing printed & nothing printed & 159 & 6.4 \\
12 & nothing printed & nothing printed & 162 & 4.9 \\
10 & nothing printed & nothing printed & little & little printed \\
& & & printed & \\
8 & nothing printed & nothing printed & nothing & nothing \\
& & & printed & printed \\
6 & nothing printed & nothing printed & nothing & nothing \\
& & & printed & printed \\
& nothing printed & nothing printed & nothing & nothing \\
& & & printed & printed \\
\hline
\end{tabular}

Table 6.1: The dimension of the hole left in the donor and the deposit on the acceptor with varying pulse energy.

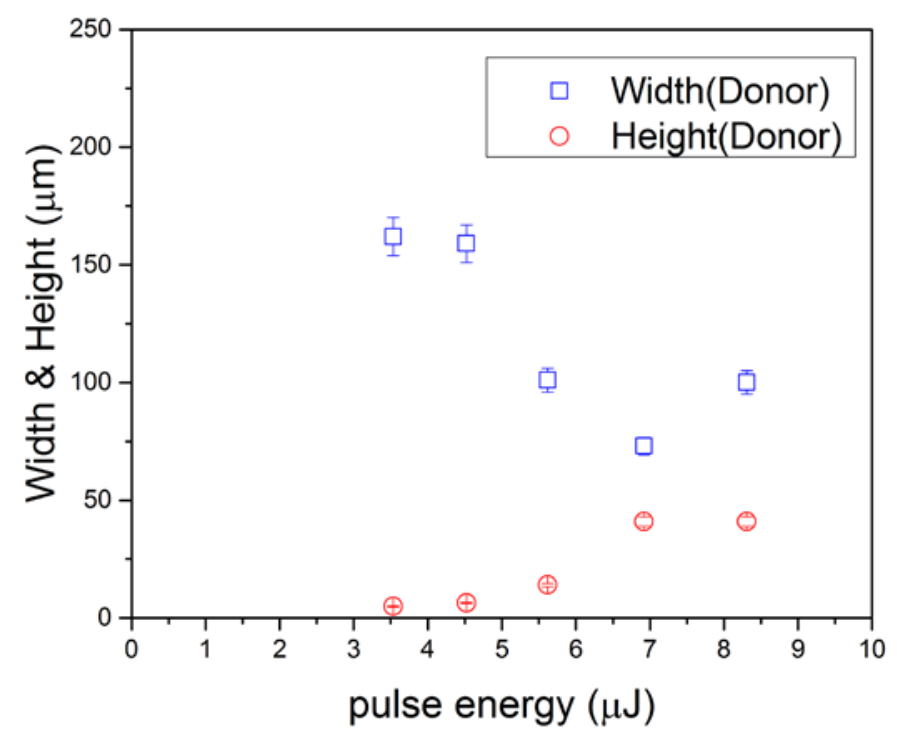

Figure 6.3: The dimension of the hole left in the donor with pulse energy varying from $0.7 \mu \mathrm{J}$ to $8.3 \mu \mathrm{J}$. 
In the nanosecond LIFT experiment, we have discussed the transfer mechanism with respect to different transfer regimes. For a given donor film thickness $(h)$, when the laser pulse fluence $F_{t h}$ is below the transfer threshold energy value $F_{t h}(h)$, the laser-induced bubble collapses and a blister forms in the paste. In the present experiment, instead of a bulge blister we observed the shallow depression region left on the donor. This indicates that when the balance between the internal and external force is broken after the bubble collapse the ambient pressure and surface tension dominate the flow direction. Therefore, the paste recoils and forms a depression in the donor. And the wider and shallower size of the hole is attributable to the surface tension of the paste.

The multiple pulse dotting has been studied in the nanosecond LIFT single voxel printing experiment. And the results demonstrate that due to the high viscosity nature of the silver paste, the silver paste has not fully recover the depression area. In the fourth test, we repeat the same idea with the ps laser using $6.9 \mu \mathrm{J}$ and $5.6 \mu \mathrm{J}$ of the pulse energy, which are around the threshold energy values, firing at the same location with a burst of pulses of which burst count is from 1 to 10 . The results are studied with the dimensions of the hole and the voxel on both donor and acceptor substrates.

\begin{tabular}{ccccc}
\hline & Acceptor (Voxel) & & \multicolumn{2}{c}{$\begin{array}{c}\text { Donor } \\
\text { (hole) }\end{array}$} \\
\hline $\begin{array}{c}\text { Number } \\
\text { of pulse }\end{array}$ & Width $(\boldsymbol{\mu m})$ & Height $(\mu \mathrm{m})$ & $\begin{array}{c}\text { Width } \\
(\boldsymbol{\mu m})\end{array}$ & Depth $(\boldsymbol{\mu m})$ \\
1 & nothing printed & nothing printed & 93 & 9 \\
2 & nothing printed & nothing printed & 94 & 10 \\
5 & little printed & little printed & 98 & 26 \\
10 & ablation & ablation & 94 & 35 \\
\hline
\end{tabular}

Table 6.2: The dimension of the hole left in the donor and the deposit on the acceptor with pulse energy of $6.9 \mu \mathrm{J}$. 


\begin{tabular}{ccccc}
\hline \multicolumn{3}{c}{ Acceptor(Voxel) } & \multicolumn{3}{c}{ Donor(hole) } \\
\hline Number of pulse & Width $(\mu \mathrm{m})$ & Height $(\mu \mathrm{m})$ & Width $(\mu \mathrm{m})$ & Height $(\mu \mathrm{m})$ \\
1 & nothing printed & nothing printed & 97 & 5 \\
2 & nothing printed & nothing printed & 98 & 5 \\
5 & little printed & ablation & 97 & 5 \\
10 & 97 & ablation & 100 & 37 \\
\hline
\end{tabular}

Table 6.3: The dimension of the hole left in the donor and the deposit on the acceptor with pulse energy of $5.6 \mu \mathrm{J}$.
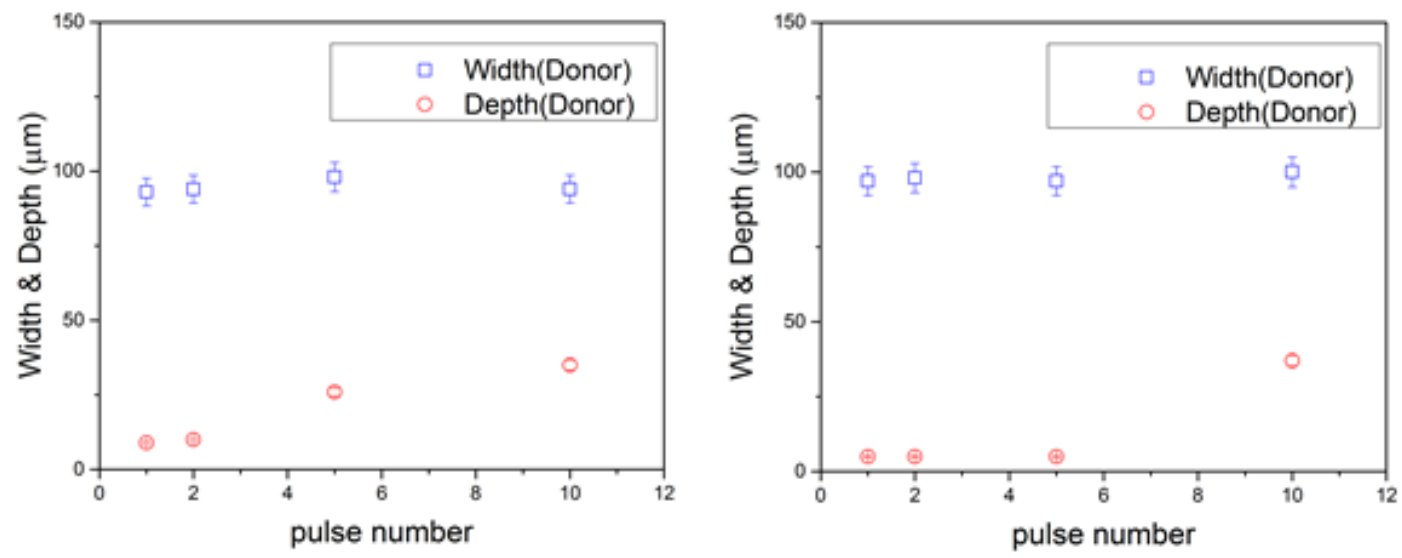

Figure 6.4: The width of the hole left in the donor with pulse energy of 5.6 (left) and 6.9 (right) $\mu \mathrm{J}$.

From Table 6.2 and Table 6.3 it is observed that with the threshold energy and burst count from 1 to 5 , there is almost no paste transferred to the acceptor and from the burst of 10 pulses the laser starts to ablate the acceptor. This result is coincident with the one obtained with nanosecond laser. And Fig. 6.4 shows the sizes of the holes left on the donor. The width decreases as a function of the pulse number and finally the value remains constant, while the depth slightly increases with pulse number. The constant value of the dimension of the hole indicates that the laser has cleaned off the hole. On the other hand, a circular region formed by a large quantity of droplets is observed on the acceptor substrate. The diamter of the circular area increases with pulse numbers. Single 
laser pulse does not make the paste transfer since the protrusion front of the blister doesn't touches the acceptor. The consequent pulses vaporize more organic components in the paste and push a portion of paste toward the acceptor. Then the following pulses will disaggregate the dropping fragments of paste and finally make the explosive transfer (Fig. 6.5).
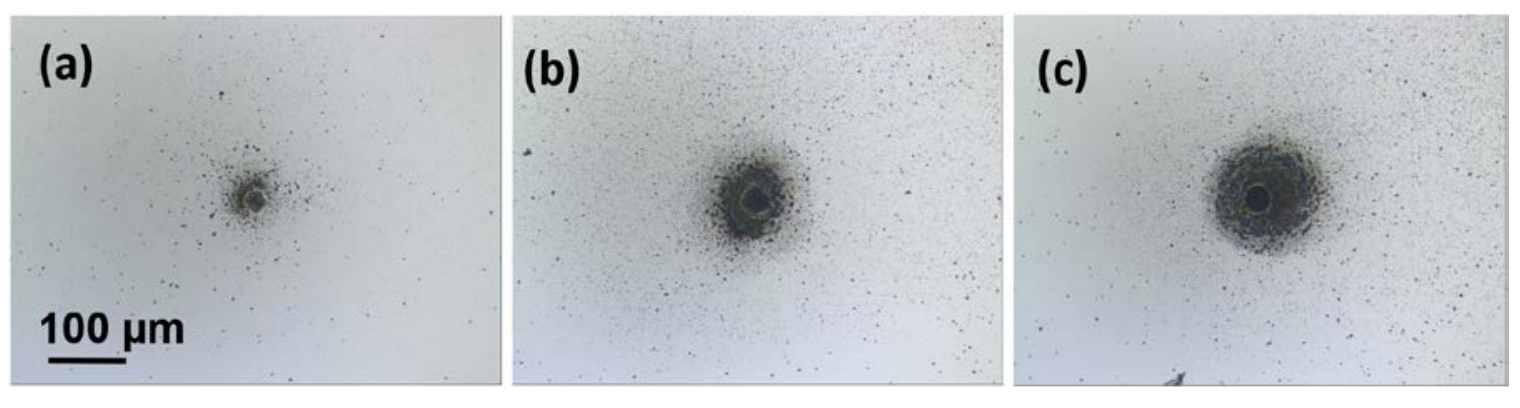

Figure 6.5: Microscope images of picosecond LIFT silver paste with different burst counts: (a) 10, (b) 50, (c) 500 .

The results indicated that with a burst of pulses of which energies below the threshold value the transferred silver paste spreads explosively and cannot form a concrete dot.

\subsubsection{Comparison with different Gap distance}

Now the GAP distance is reduced to $50 \mu \mathrm{m}$ and made a comparison with the 100- $\mu \mathrm{m}$-gap experimental results.

Fig. 6.6 shows microscopic images of dots transferred with three different laser fluences and two gap distances. When using $100 \mu \mathrm{m}$ gap leads to a larger transferred area which covered by disaggregated droplets on the acceptor substrate. At the low energy $(8.3 \mu \mathrm{J})$ the cluster transfer is observed, in which the voxel comprises non-continuous clusters of paste. However, as increasing the laser fluence, the dispersion area in which the transferred voxels exhibits splashing behavior with a great number of droplets strongly increases. 
As a comparison, when using a smaller gap $(50 \mu \mathrm{m})$, there is less dispersion of the paste. This leads to the concrete-dot transfer, which can produce a single, well-defined paste dot using the threshold fluence $(8.3 \mu \mathrm{J})$. With this transfer mechanism, larger aspect ratio voxel and uniform line can be printed by LIFT. Whereas, as increasing the fluence, the voxel size enlarges remaining a central concrete part with small dispersions at the surroundings.

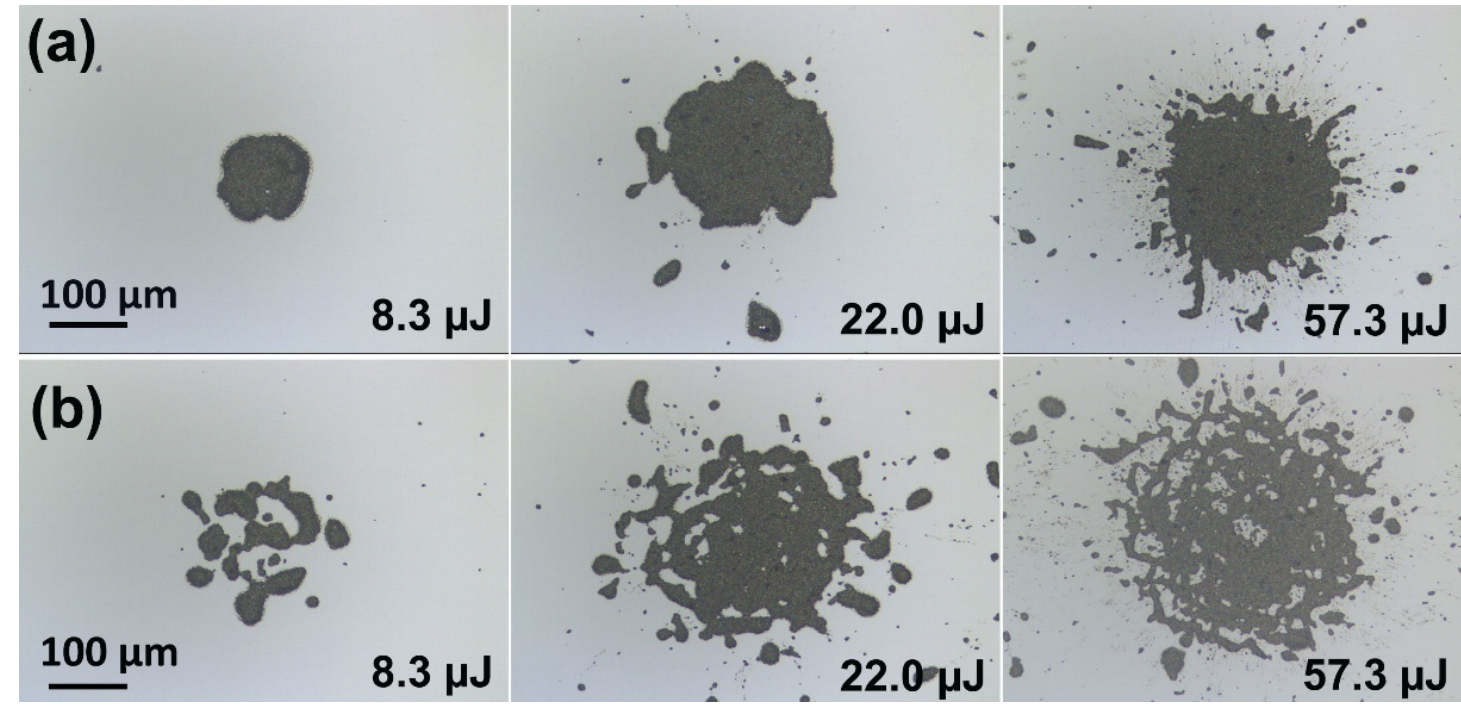

Figure 6.6: Microscope images of LIFT dots transferred using single shots of ps laser and with fluence varying from 8.3 to $57.3 \mu \mathrm{J}$ with two different gap distance: (a) $50 \mu \mathrm{m}$ and (b) $100 \mu \mathrm{m}$.

Fig. 6.7 shows the transferred voxel size (height, width and aspect ratio) as a function of the fluence for the two gap distances. Each data point represents the average of measuring 5 different voxels. The width of the voxel is defined as the diameter of the compact circular-like region excluding the splashed small droplets, and the height is defined as the average value of the measured profile. With both gap distance, the width of the voxels increases as a function of laser pulse energy in a range between 8.3 and 57.3 $\mu \mathrm{J}$ and finally saturates, whereas the height shows a downward trend. In the case of the $50 \mu \mathrm{m}$ gap, the transferred dots are higher and narrower. Fig. $6.7 \mathrm{c}$ shows the aspect ratio of the dots transferred using both gaps. The aspect ratio of the dots transferred using 50 
$\mu \mathrm{m}$ is larger than those with longer gap distance $(100 \mu \mathrm{m})$. Moreover, it increases as the pulse energy decreases, up to 0.09 when using the lowest pulse energy.

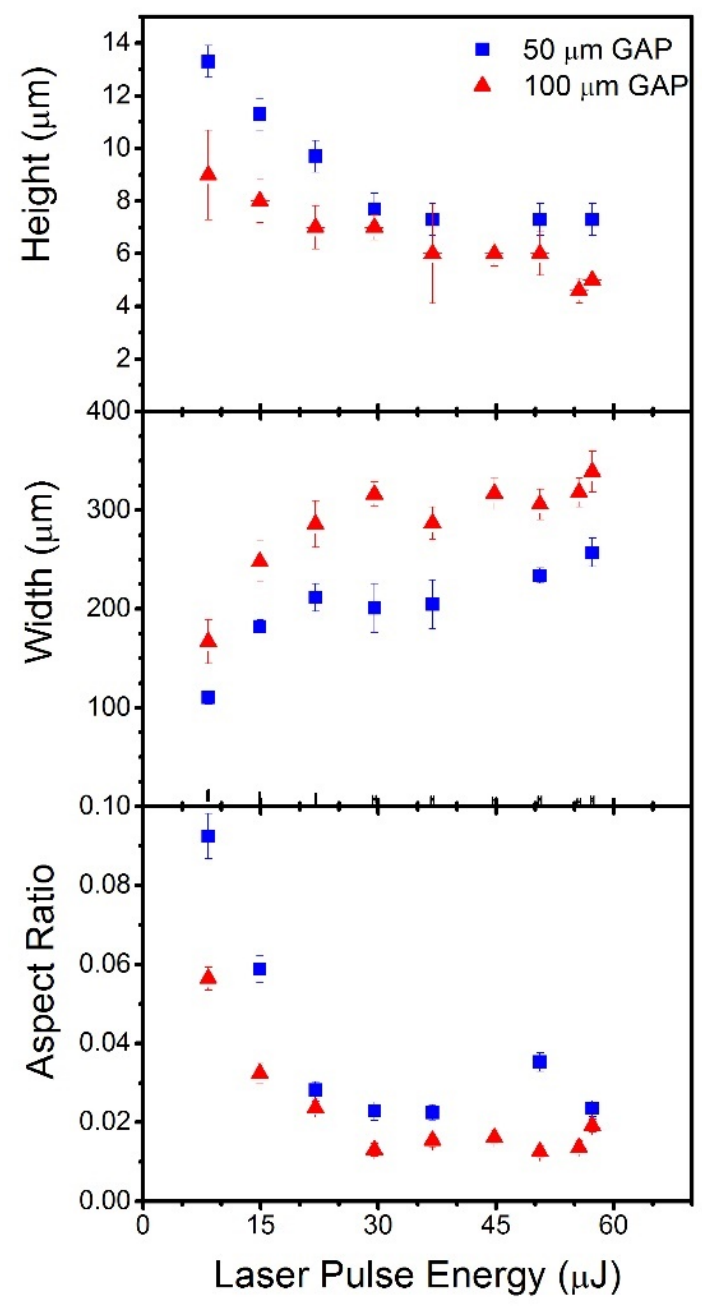

Figure 6.7: Height, width and aspect ratio of the transferred dots as a function of fluence varying from 0.7 to $57.3 \mu$ with respect to two different gap distance (50 $\mu \mathrm{m}$ and 100 $\mu \mathrm{m})$.

Fig. 6.8 demonstrates the dimension of the hole left in the donor and the voxel deposited on the acceptor with GAP distance of $50 \mu \mathrm{m}$. It can be seen that both widths of the hole and the voxel increase as a function of the laser pulse energy, which is in 
agreement with the results obtained with the GAP distance of $100 \mu \mathrm{m}$. And the depths of the hole remain a constant of the layer thickness, which indicates that the laser cleaned off the hole when using the pulse energy above the transfer threshold value.
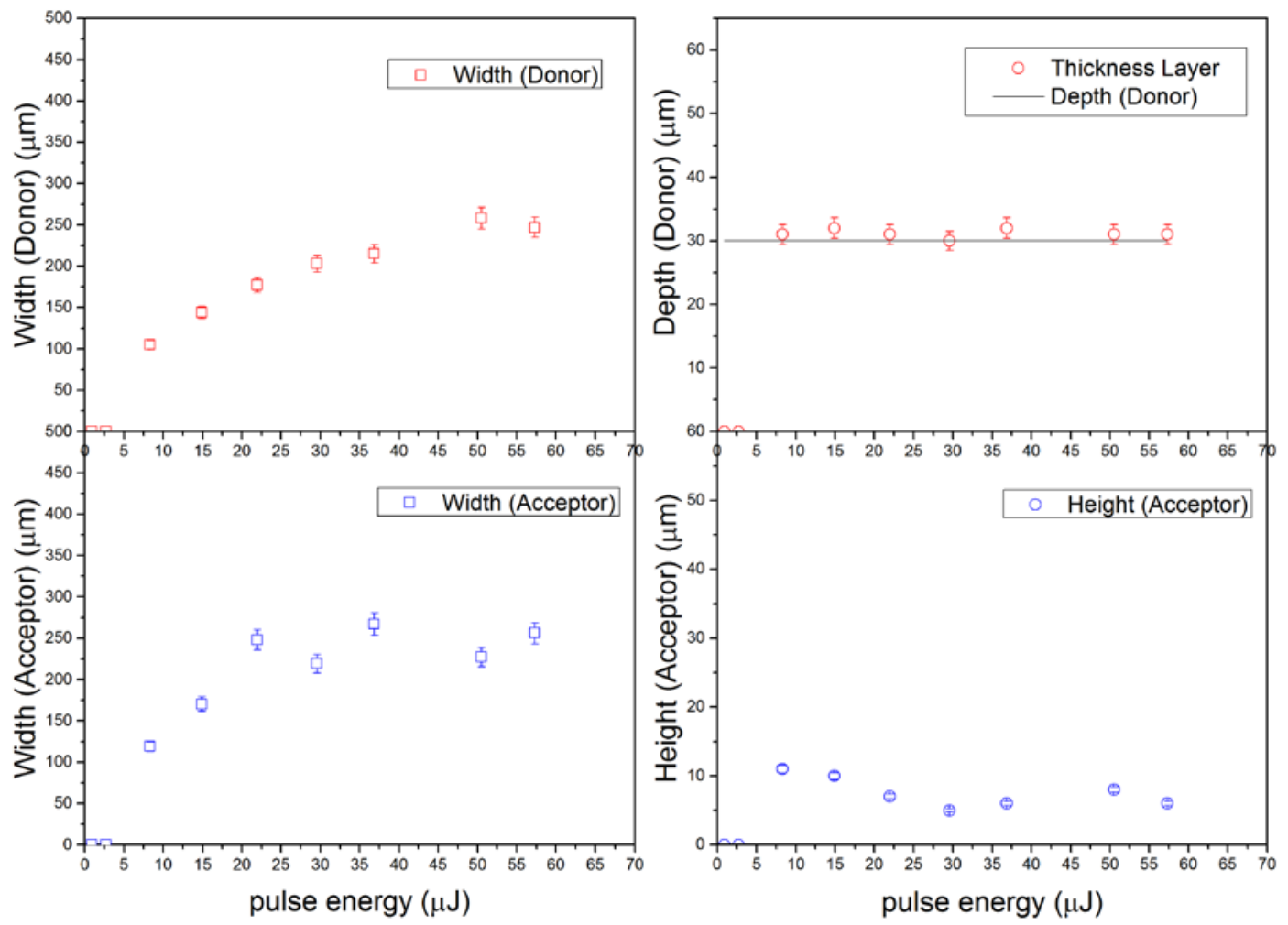

Figure 6.8: The dimension of the hole left in the donor and the voxel deposited on the acceptor with GAP distance of $50 \mu \mathrm{m}$.

Fig. 6.9 shows the morphology of the best voxel obtained, printed using the thinner donor film thickness $(50 \mu \mathrm{m})$ and pulse energy just above transfer threshold $(8.3 \mu \mathrm{J})$. These voxels consist of single well define dot of paste, without any splashing or disaggregated cluster of paste. The aspect ratio (height divided by width) is also the largest obtained (0.09). Since the application of this paste is intended for flexible electronics and solar cell metallization, the figure-of-merit of the process should be the aspect ratio, defined as the ratio between the height and the width: the bigger the height 
the lower the electrical resistance of a transferred line, while the narrower the width the smaller the area occupied by the line in the final device.

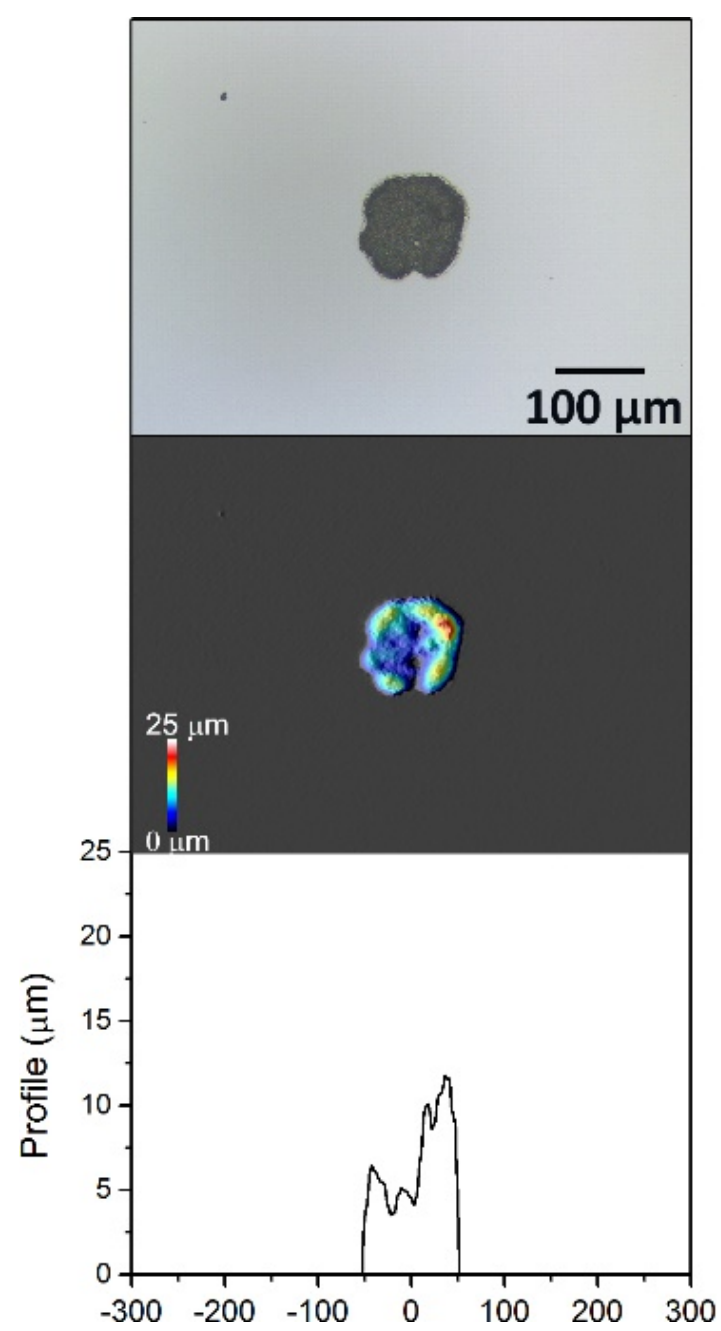

Figure 6.9: The morphology of the best voxel using a donor film thickness of $50 \mu \mathrm{m}, 50$ $\mu \mathrm{m}$ gap distance and pulse energy threshold of $8.3 \mu \mathrm{J}$.

\subsection{Picosecond LIFT lines}

The previous experimental parameters are the starting point for printing lines. Continuous lines or more complex 3D structures can be printed with these parameters by either scaninng the laser beam or moving the sample for overlapping different voxels. In this work, the beam position has been fixed and the sample has been moving using a 
motorized $X-Y$ stage. The stage moving speed has been fixed at $60 \mathrm{~mm} / \mathrm{s}$ and the laser repetition rate were varied from 400 to $2000 \mathrm{~Hz}$. Hence, the pitch distance studied was from $30 \mu \mathrm{m}$ to $150 \mu \mathrm{m}$. The sample started moving prior to fire the laser, avoiding any accelaration effect in the beginning or end of the line.

In the line printing section, we fixed the energy at $8.3 \mu \mathrm{J}$ and made a line test with different spot separation distance related with different repetition frequency values. The result showed that with $100 \mu \mathrm{m}$ of separation distance the morphology is the best one. Then we fixed the spot separation distance at $100 \mathrm{um}$ and varied the energy from $24 \%$ to $16 \%$ attenuation of the full range energy to make lines of $3 \mathrm{~cm}$. The best results were obtained with an attenuation of $11.2 \mu \mathrm{J}$.

\subsubsection{Influence of the pitch distance}

In this section the influence of the pitch distance on the LIFT process is presented. Here the pitch distance is defined by the distance between single successive laser pulses. And this pulse-to-pulse distance is directly determined by dividing the stage moving speed and the laser pulse repetition rate. It is also regarded as one of the key parameter for the succesful formation of lines and patterns of LIFT of silver paste since the line is constructed by overlapping each single voxel with a certain range of distance. And this heart-to-heart distance between two succesive voxel is related with the pitch distance.

Fig. 6.10 shows the false color height map of both donor (Fig. 6.10b) and acceptor substrate (Fig. 6.10d) and the corresponding cross-section profile (Fig. 6.10c) for different pulse overlapping distances. Fig. 6.10a shows the scheme of the temporal pulse train of laser pulses, i.e. when and how many laser pulses have been fired. The dash lines in Fig. $6.10 \mathrm{c}$ indicates the interface between the silver paste and the glass slide.

In the case of lines with pitch distance of $150 \mu \mathrm{m}$ or $100 \mu \mathrm{m}$, laser pulses cleaned off all the paste from the donor substrate, leaving a hole being its bottom the surface of the glass slide and its depth is the real thickness of the paste film in that point. The depth of the remaining hole decreases as the overlapping distance is reduced. In the case of a 60 
$\mu \mathrm{m}$ pitch distance, the holes are hardly observed. The size of the bulge decreases also as the pitch distance decreases. Those bulges are due to the displacement of silver paste, which is not transfer but recoil to the border of the hole.

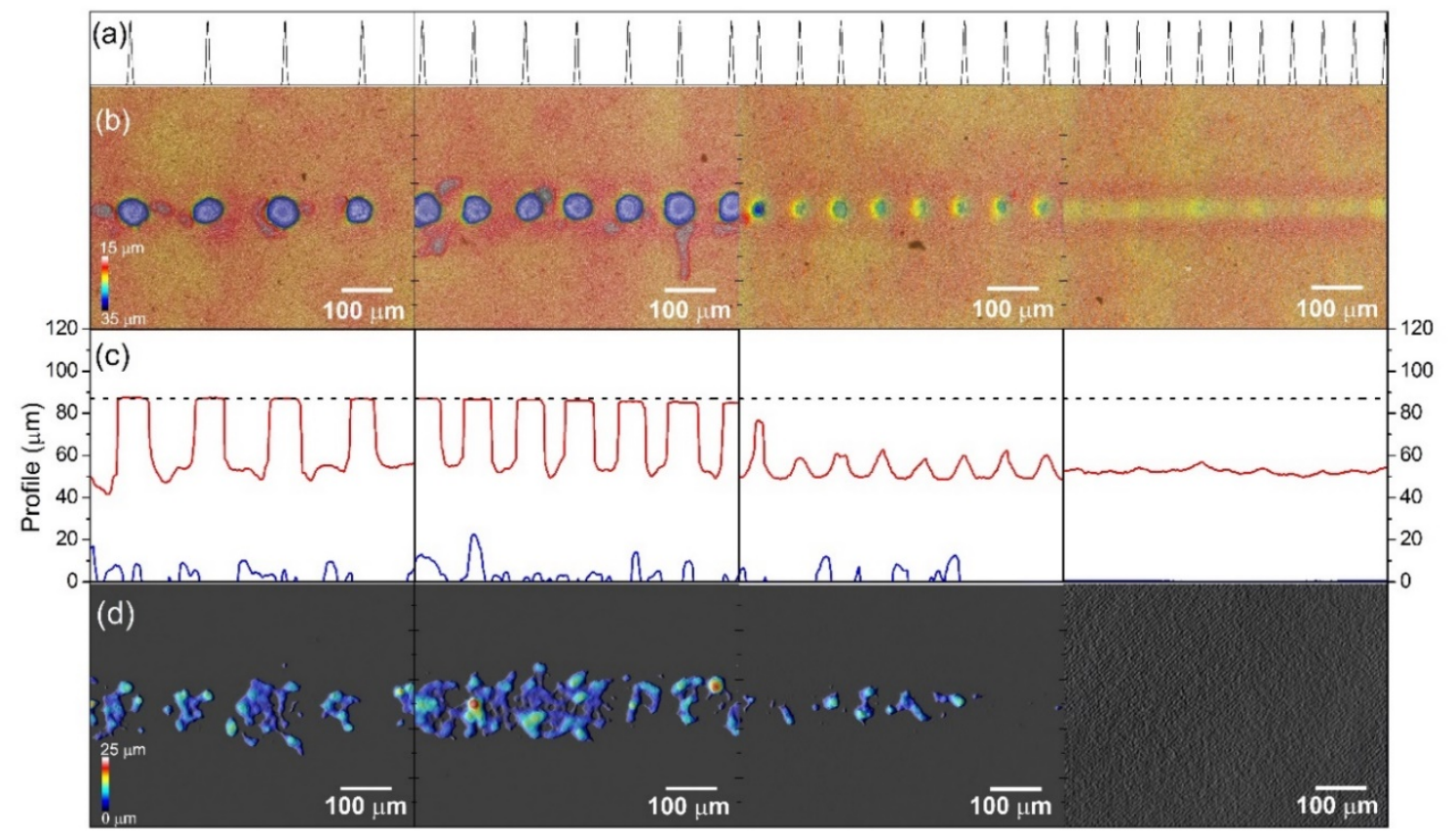

Figure 6.10: Confocal images of the middle part of (d) transferred line and (b) donor films with $8.3 \mu \mathrm{J}$ laser pulses, $35 \mu \mathrm{m}$ film thickness, $50 \mu \mathrm{m}$ gap distance and different pitch distances: $150 \mu \mathrm{m}, 100 \mu \mathrm{m}, 80 \mu \mathrm{m}$ and $60 \mu \mathrm{m}$ (from left to right). Laser pulse and crosssectional profile of both holes left in the donor and the transferred pastes are shown in (a) and (c).

The morphology of the transferred line is also strongly affected by the pitch distance. When using a large pitch $(150 \mu \mathrm{m})$ there is no overlapping and the voxels are clusters of dispersed droplets instead of a concrete and well-defined dot, which is transferred in the case of the single pulse experiment. When reducing the pith distance to $100 \mu \mathrm{m}$ the voxels starts to overlap and a line, although not yet continuous, is formed. However, decreasing the pith distance below $100 \mu \mathrm{m}$ yields a much less quantity of transferred paste. At the shortest pitch distance $(60 \mu \mathrm{m})$ there is even no paste transferred at all. That result was 
unexpected as it was supposed that with more number of laser pulses given to the film, i.e. larger energy, more paste should be transferred, although the morphology of the line could be worse. It is observed that successive pulses overlapped at a close distance to each other affects the transfer mechanisms in such a way that the transfer is ceased and hence there is a maximum pitch distance that acts as transfer threshold for line printing.

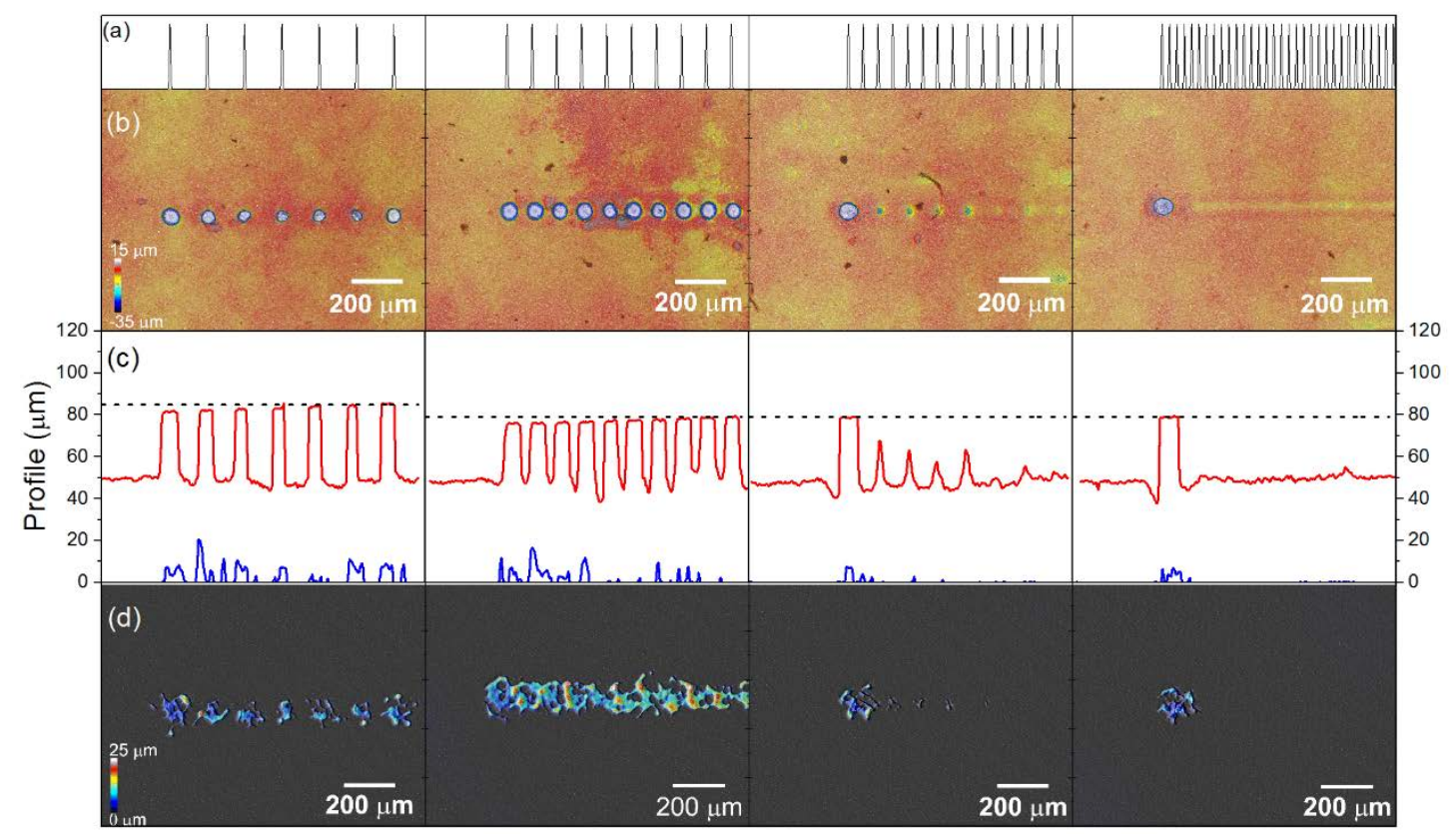

Figure 6.11: Confocal images of the beginning part of the line (a) Laser pulse (b) donor and (d) transferred line and (c) profiles in pairs of donor and acceptor with $8.3 \mu \mathrm{J}$ pulse energy with different pitch distances: $150 \mu \mathrm{m}, 100 \mu \mathrm{m}, 60 \mu \mathrm{m}$ and $30 \mu \mathrm{m}$ (from left to right). Laser beam scanning direction is from right to left.

In order to investigate the effect of overlapping on the transfer mechanism, the beginning part of each line was also characterized by confocal microscopy. The same scheme of Fig. 6.10 is used in Fig. 6.11 illustrating the temporal train of laser pulses (Fig. 6.11a), the false color height map of both donor (Fig. 6.11b) and acceptor substrate (Fig. 6.11d) and the corresponding cross-section profile (Fig. 6.11c) for different pitch distances. 
The hole left in the donor substrate and the voxel transferred by the very first laser pulse is approximately the same in every pitch distance. That is because this first laser in each line pulse found fresh material, not affected by previous pulses. Lines printed with a pitch distance of 150 and $100 \mu \mathrm{m}$ show the same trend in the beginning than in the middle of the line (Fig. 6.10). However, when using a pitch distance of $60 \mu \mathrm{m}$, the depth of the holes in the donor starts to decrease along the direction of the scanning laser pulse trace after the first pulse, while the size of the transferred voxel decreases dramatically along that direction. In the case of the shortest pitch distance shown in Fig. $6.11(30 \mu \mathrm{m})$ there is only one hole in the donor substrate, followed by a shallow groove, and only the voxel corresponding to the first pulse is transferred.

\subsubsection{Influence of the laser pulse energy}

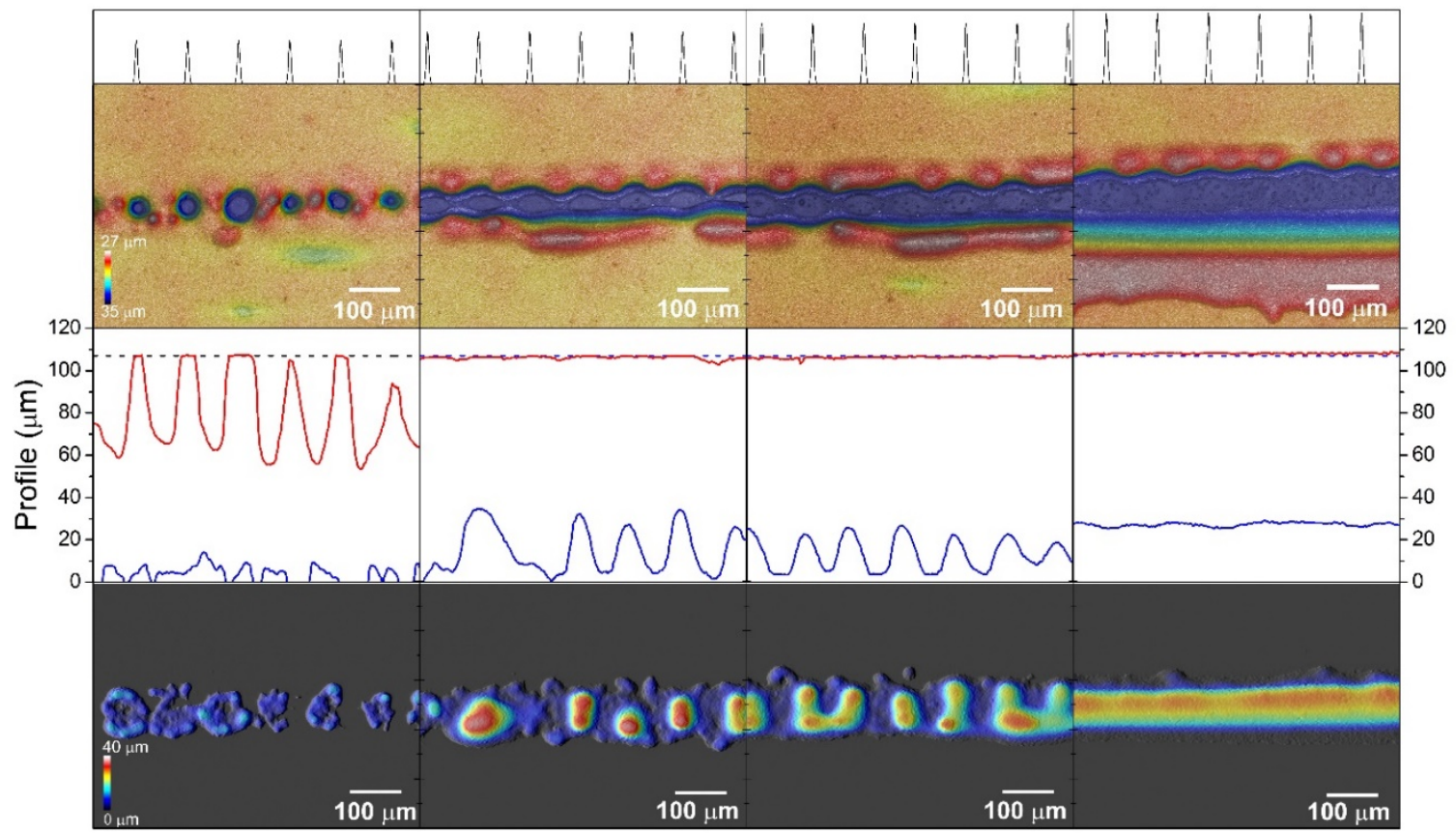

Figure 6.12: Confocal images of the middle part of the line (a) Laser pulse (b) donor and (d) transferred line and (c) profiles of both holes left in donor and transferred line on the acceptor with pitch distance of $100 \mu \mathrm{m}$ and different laser pulse energies: 5.6, 8.3, 9.7 and $11.2 \mu \mathrm{J}$ (from left to right). 
The overlapping of the voxels is not only determined by the pitch distance but also by the size of the transferred voxel. According to the results shown above the best pitch distance is $100 \mu \mathrm{m}$, in which almost continuous lines are printed. Therefore, we slightly vary the pulse energy and increase the film thickness to $40 \mu \mathrm{m}$ in order to increase the voxel size and to improve the morphology of the line. Fig. 6.12 shows the false color height map of both donor (Fig. 6.12b) and acceptor substrate (Fig. 6.12d) and the corresponding cross-section profile (Fig. 6.12c) of four lines printed using pulse energies from 5.6 to 11.2 $\mu \mathrm{J}$.

In the case of the lowest laser fluence, $5.6 \mu \mathrm{J}$, the pulses are separated and neither the holes left in the donor nor the transferred voxels are overlapped. As the energy increases the size of the hole increases, forming a continuous, paste clean groove. The width of the groove increases as the pulse energy increases. Voxels increases their height but not their width in the transversal direction to the line as the pulse energy increases. The continuity of the line also improves until it is finally possible to transfer a several cmlong continuous line when using a laser fluence of $11.2 \mu \mathrm{J}$.

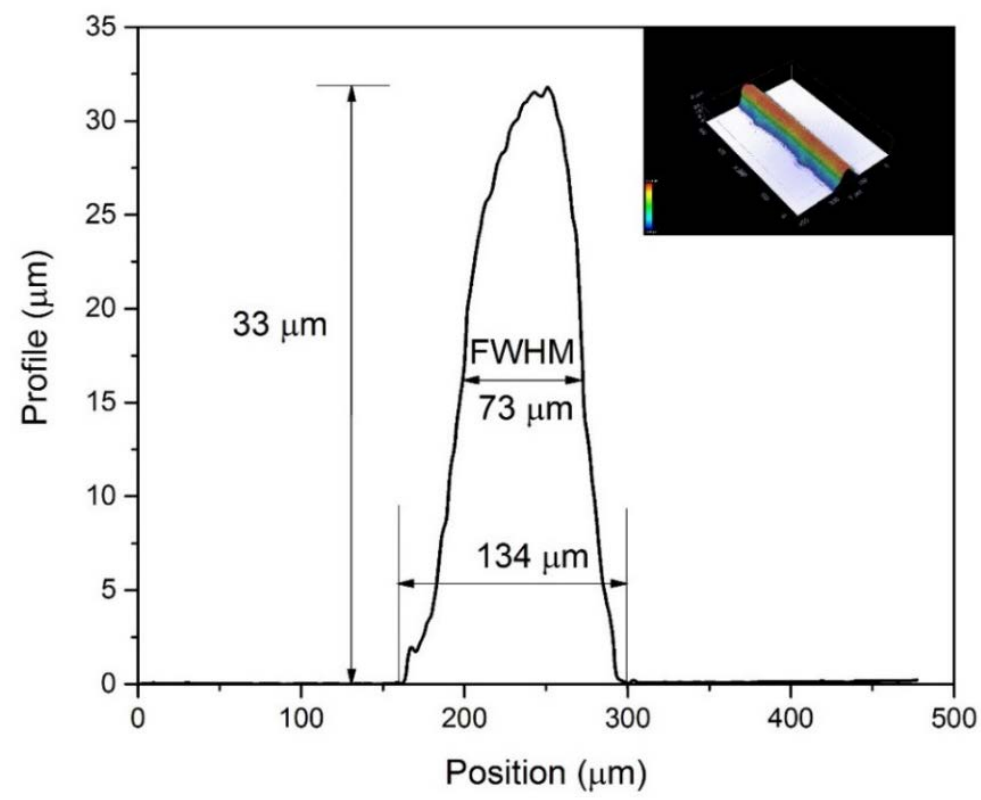

Figure 6.13: Cross-section profile of the printed line with $40 \mu \mathrm{m}$ film thickness, $50 \mu \mathrm{m}$ gap distance and laser pulse energy of $11.2 \mu$. 
Fig. 6.13 represents the Gaussian shape cross-section profile of the optimum line. It has a large aspect ratio of 0.25 with a cross-section area of $2425 \mu \mathrm{m}^{2}$. The width of the line is $134 \pm 2 \mu \mathrm{m}$ and the height of the line is $33 \pm 1 \mu \mathrm{m}$ (each value represents the average of 5 measurements with an error denoting one standard deviation). With such a profile, the ps LIFT lines can provide better conductivity for the front contact metallization.

\subsubsection{Lines drawn using polygon scanner}

In order to test the LIFT process in very high scanning speed, the EKSPLA Atlantic laser is equipped with the polygon scanner. Parameterization have been done by directly write the lines with fixing the gap distance $(50 \mu \mathrm{m})$ and the focal position. A repetition rate of $598 \mathrm{kHz}$ and a scanning speed of $59.8 \mathrm{~m} / \mathrm{s}$, which leads to the optimum pitch distance of $100 \mu \mathrm{m}$. Fig. 6.14 illustrates the lines transferred using different pulse energies. The optimum one is the line transferred using $18.1 \mu \mathrm{J}$, which has an aspect ratio of 0.12 (Fig. $6.15)$.

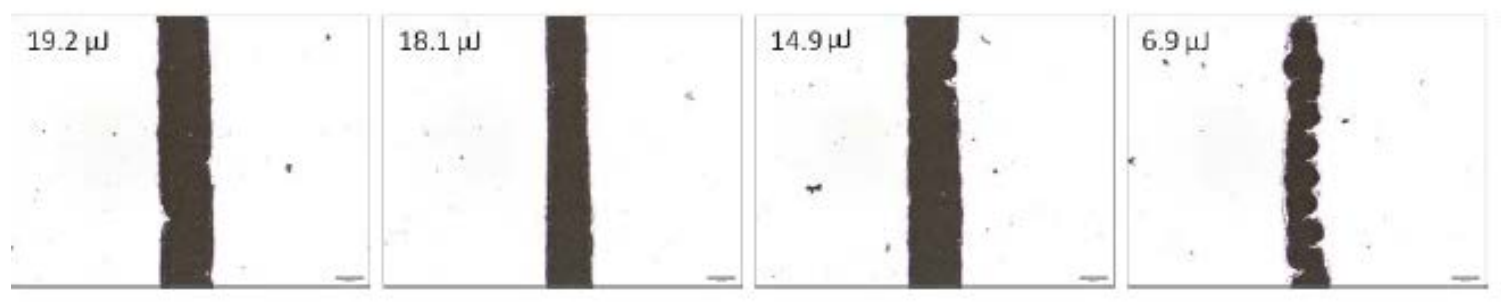

Figure 6.14: Microscope images of LIFT line transferred with different pulse energies by using polygon scanner.

\subsubsection{Influence of the laser wavelength}

In this section, a ps laser with $1064 \mathrm{~nm}$ was employed to have a comparison with the $532 \mathrm{~nm}$ results.

The first experiment is to fix the laser energy at $8.8 \mu \mathrm{J}$ and vary the scan speed from 100 to $10000 \mathrm{~mm} / \mathrm{s}$ of which pitch distance is from 2 to $200 \mu \mathrm{m}$. The gap distance and 
film thickness is the same as used in the last section. The LIFT process parameters are listed in Table. 6.4.

\begin{tabular}{|c|c|}
\hline LIFT parameters & Value \\
\hline Laser Repetition Rate & $50 \mathrm{Khz}$ \\
\hline Pulse width & $32 \mathrm{ps}$ \\
\hline Numbers of line & 9 \\
\hline Scanning speed & $100-10000 \mathrm{~mm} / \mathrm{s}$ \\
\hline Pitch & $2-200 \mu \mathrm{m}$ \\
\hline GAP & $50 \mu \mathrm{m}$ \\
\hline film thickness & $40 \mu \mathrm{m}$ \\
\hline Focus position & $6.8 \mathrm{~mm}$ \\
\hline Line length & $3 \mathrm{~cm}$ \\
\hline Pulse energy & $5.8-13.6 \mu \mathrm{J}$ \\
\hline
\end{tabular}

Table 6.4: LIFT process parameters with ps-pulsed laser at $1064 \mathrm{~nm}$.

Fig. 6.15 represents the influence of the pitch distance using $1064 \mu \mathrm{m}$ ps laser. It can be seen that at $2 \mu \mathrm{m}$ pitch distance there is a trace of explosive powders on the acceptor. From 10 to $40 \mu \mathrm{m}$, there is rarely no paste transfer except for the beginning part. And above $80 \mu \mathrm{m}$ the cluster-dot transfer starts to appear. When the pitch distance increased to $200 \mu \mathrm{m}$, the concrete-dot transfer is observed. This pitch distance effects on the LIFT process using $1064 \mu \mathrm{m}$ are the same as those observed with the $532 \mu \mathrm{m}$ ps laser. 


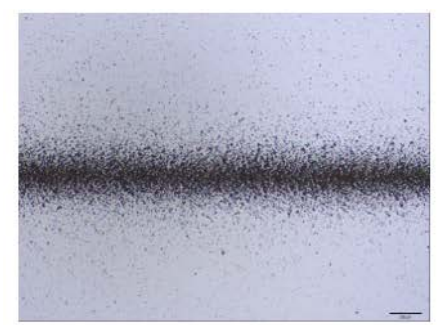

$100 \mathrm{~mm} / \mathrm{s}(2 \mathrm{um})$

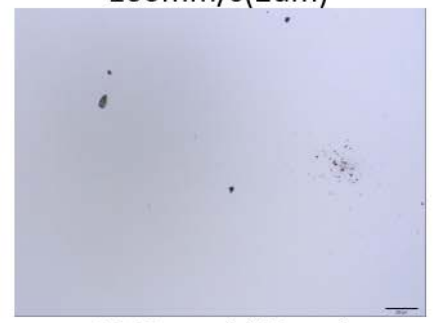

$2000 \mathrm{~mm} / \mathrm{s}(40 \mathrm{um})$

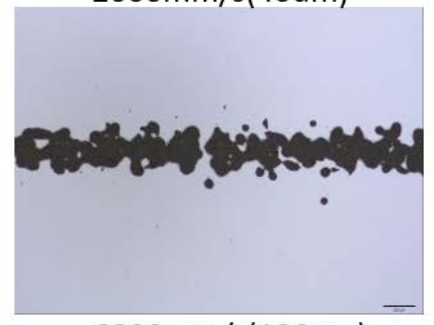

$6000 \mathrm{~mm} / \mathrm{s}(120 \mathrm{um})$

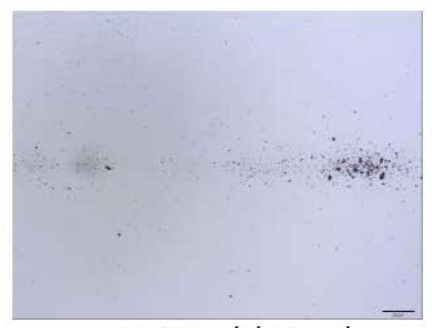

$500 \mathrm{~mm} / \mathrm{s}(10 \mathrm{um})$

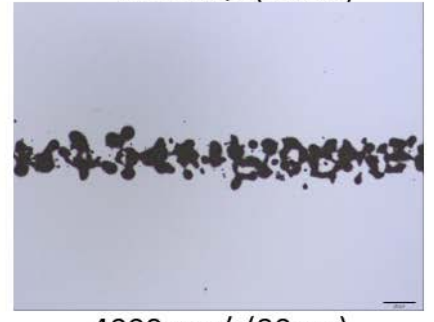

$4000 \mathrm{~mm} / \mathrm{s}(80 \mathrm{um})$

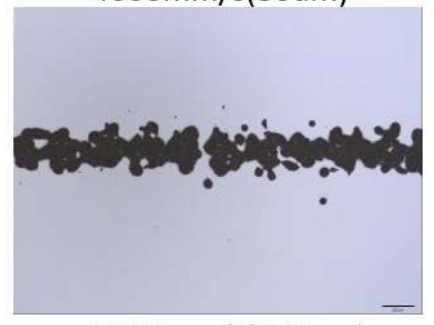

$7000 \mathrm{~mm} / \mathrm{s}(140 \mathrm{um})$

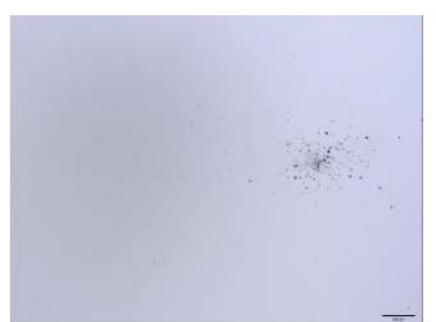

$1000 \mathrm{~mm} / \mathrm{s}(20 \mathrm{um})$

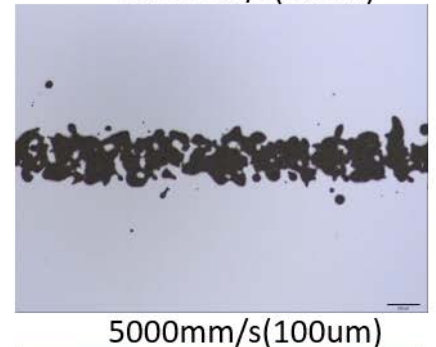

$5000 \mathrm{~mm} / \mathrm{s}(100 \mathrm{um})$

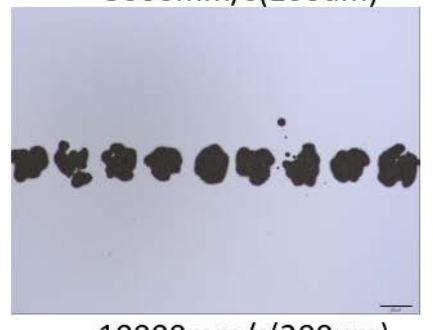

$10000 \mathrm{~mm} / \mathrm{s}(200 \mathrm{um})$

Figure 6.15: Microscope images of LIFT line transferred using $1064 \mu \mathrm{m}$ ps laser with 8.8 $\mu \mathrm{J}$ pulse energy with varied pitch distances from 2 to $200 \mu \mathrm{m}$.
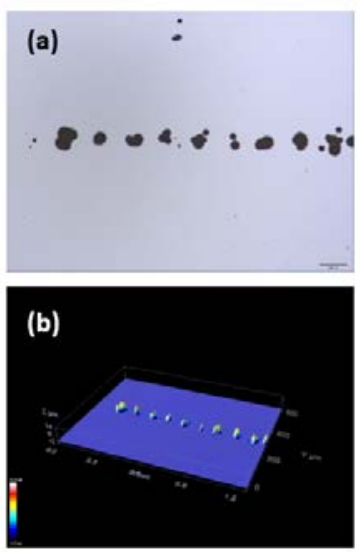

$5.8 \mu$
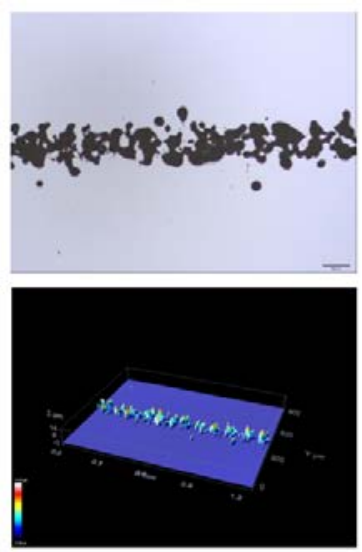

$8.8 \mu$
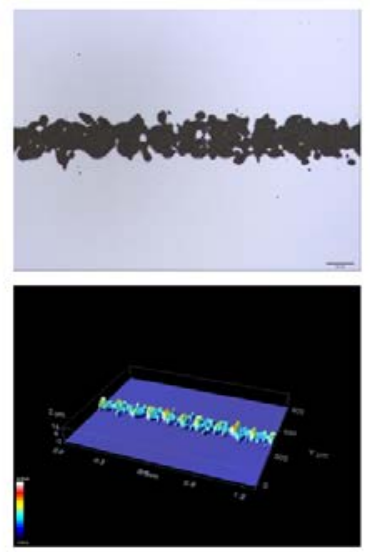

$9.9 \mu$
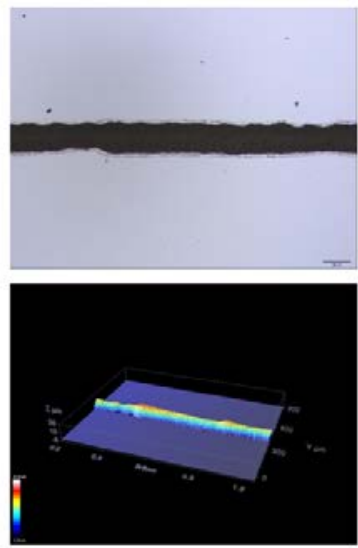

$11.4 \mu$

Figure 6.16: Microscope and Confocal images of the middle part of the transferred line on the acceptor with pitch distance of $100 \mu \mathrm{m}$ and different laser pulse energies: 5.8, 8.8, 9.9 and $11.2 \mu \mathrm{J}$ (from left to right). 
As it has been shown before, the optimum typical voxel overlapping distance is of the order of $100 \mu \mathrm{m}$, which depends on the voxel size. Fig. 6.16 shows the transferred lines on the acceptor with $100 \mu \mathrm{m}$ and different laser pulse energies $(5.8,8.8,9.9$ and $11.2 \mu \mathrm{J})$. In the case of lines printing using a fix optic, the sample is scanned by means of moving it using the $X Y$ motorized axis. Even using the minimum Repetition rate of the Katana laser $(30 \mathrm{kHz})$, the axis should move at speeds in the order of $\mathrm{m} / \mathrm{s}$. Such fast speeds become a problem that can be hardly overcome.

In LIFT, the donor substrate simply lies over the acceptor substrate and it is not fixed in any way, because it is necessary to easily remove it after LIFT processing. Thus, the largest speed in which is possible to move the system donor - acceptor substrates is in the order of $100 \mathrm{~mm} / \mathrm{s}$. Larger speeds displace the donor substrate from its position over the acceptor, ruining the overlapping and the printed line. Therefore, we have test the printing of a line using the fix focusing lens, the lowest Repetition rate possible $(30 \mathrm{kHz})$ and the largest $X Y$-axis speed without affecting the donor position $(100 \mathrm{~mm} / \mathrm{s})$.

When moving at $100 \mathrm{~mm} / \mathrm{s}$ and firing the laser at $30 \mathrm{kHz}$, the overlapping is only 3 $\mu \mathrm{m}$, what implies that a large number of pulses are fired in almost the same position. This causes the paste to be transferred in an explosive way, pulverizing it and avoiding the printing a line with good shape (Fig. 6.17). Therefore, it is necessary to use an optical scanner for moving fast the beam (at $\mathrm{m} / \mathrm{s}$ ) while keeping the donor and acceptor substrate standing still.

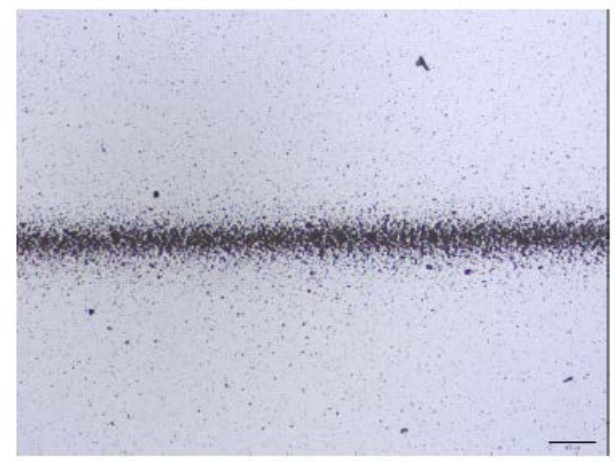

Figure 6.17: Microscope and Confocal images of the middle part of the transferred line on the acceptor with pitch distance of 100. 


\subsubsection{Discussion}

The transfer mechanisms of LIFT are quite different when using as a donor Newtonian liquids or low viscosity inks than when using a paste with a large viscosity and comprising $\mathrm{Ag}$ particles with sizes in the order of several $\mu \mathrm{m}$ [19]. In the latter case, it has been shown that the transfer mechanisms depend on the relation between laser pulse energy, donor film thickness and gap distances. When using too large energies explosive transfer occur and the paste splashes on the acceptor. If the energy is lower, typically the resulting voxel consist on small clusters of paste and only when the donor film thickness is similar to the gap and with a pulse energy near the transfer threshold is possible to transfer welldefined single dot of paste with large aspect-ratio (concrete-dot transfer). In that situation, the protruding jet touches the acceptor substrate before disaggregation allowing the formation of a stable paste pillar between donor and acceptor.

In the present work, the optimum transfer, i.e. the dot with the largest aspect ratio (0.09), the donor film thickness is slightly smaller than the gap distance. The voxel consists of a single dot of paste without disaggregation in several clusters. However, the distribution of paste is not homogenous. Thus, the transfer mechanism should in the limit of the conditions for the formation of a stable pillar. Using larger or lower pulse energies lead to respectively cluster-transfer or no-transfer. These conditions have been selected as starting point for optimization of printing of lines.

In the case of lines, a strong influence of the separation of the subsequent dots (pitch distance) on the morphology of the line has been shown. It is worth to note that the size of the laser focused beam $(\sim 10 \mu \mathrm{m})$ is one order of magnitude smaller than the diameter of the hole remaining and that of diameter of the voxel transferred (both in the order of $100 \mu \mathrm{m})$ and smaller than the pitch distance $(50-150 \mu \mathrm{m})$. So when the term overlapping is used, it refers to the overlapping of the imprint left in the donor (hole or not) or to the overlapping of the printed voxels. 
In the case of the largest pitch distance $(150 \mu \mathrm{m})$, it should be large enough to avoid any interaction between subsequent dots and then the voxels should be similar to the static process. However, the voxels consist of several clusters instead of a well-define single dot of paste. In the case of the shortest pitch distances (shorter than $30 \mu \mathrm{m}$ ) the interaction effect is even more accused: the shorter the pitch distances the less amount of material transferred. The beginning of the line shows that the very first pulse is the same independent of the pitch distance. These first voxels have always the same cluster structure.

When printing lines with Newtonian liquids by LIFT, the shift of pitch distance (center to center distance) also affects the formation of the line uniformity and continuity [106, 107]. When the pitch distance is shorter than the diameter of the droplet, coalesce appears which results in a dashed line. With even more shorter pitch distance, bulges are observed in the line but with good continuity.

\section{$\underline{\text { Explanation of cluster transfer }}$}

In the case of the high viscosity paste, the situation could be understood as an intermediate one. When the first laser pulse is fired, the pillar is formed between donor film and acceptor substrate. There is a flow of the surrounding paste but there is a hollow remaining in the donor film.

Time between pulses is in the order of several ms, longer than the typical times needed to the displacement of the paste, the formation of the protruding jet or the formation of the stable pillar (in the order of tens of $\mu \mathrm{s}$ ) [19]. Once one pillar is formed ant the next laser pulse is fired, the expanding vapor bubble affects the delicate equilibrium of the previous system bubble-pillar, breaking the stable pillar, and projecting fragments of paste onto the acceptor in the shape of small clusters. 


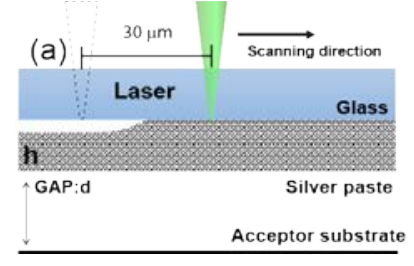

(b)

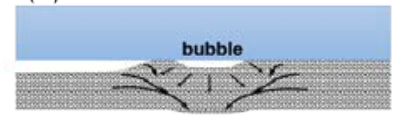

(c)

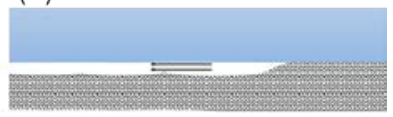

Figure 6.18: Schematic of the laser pulse overlapping effect with short pitch distance on the donor substrate in the LIFT process.

\section{Effect of short pitch distance}

If the pitch distance is shorter than the hole size, the second pulse is fired in a region without paste because the previous shot has cleaned off the paste. Logically, if there is not any paste to ablate, the gas bubble is not generated and there is no transfer of material (see Fig. 6.18). When the pitch distance is slightly larger, the bubble is generated. However, the paste wall between the bubble and the previous hole is very thin. The large pressure generated inside the bubble could break that wall, reducing the pressure. Therefore, the amount of material pushed to the acceptor substrate is strongly decreased (in the case of a pitch distance of $60 \mu \mathrm{m}$ ) or even leading to no transfer at all (at a pitch distances of $30 \mu \mathrm{m})$. Although there is no transfer there is bubble generation and movement of paste inside the film, leaving a void volume inside the film. The gas bubbles induced by subsequent pulses will reduce their pressure in the same way and enlarge the void volume, forming a draught tunnel along the laser track in the film.

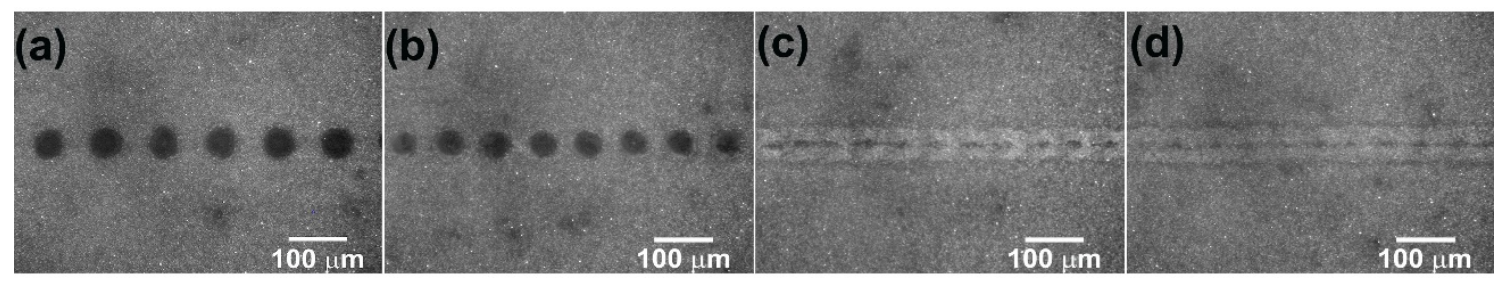

Figure 6.19: Top view microscope images through the glass of the middle parts of laser scanning trace with pitch distance of $100 \mu \mathrm{m}, 80 \mu \mathrm{m}, 60 \mu \mathrm{m}$ and $30 \mu \mathrm{m}$ (from left to right). 
To verify the proposed mechanism, we characterize the donor again by using microscope through the glass slide focusing on the film-glass interface. Figure 6.19 illustrates the top view of microscope images in the middle part of laser scanning traces left in the donors with pitch distance of $100 \mu \mathrm{m}, 80 \mu \mathrm{m}, 60 \mu \mathrm{m}$ and $30 \mu \mathrm{m}$.

In the case with pitch distance of $100 \mu \mathrm{m}$ (Fig. 6.19a), individual holes are observed. When pitch distance reduced to $80 \mu \mathrm{m}$ (Fig. 6.19b), instead of holes, craters appear near the film-glass interface, i.e., the crater formed on the paste surface and the crater left on the film-glass interface are not connected. And the diameter of these holes is smaller than the ones with pitch distance of $100 \mu \mathrm{m}$. This can be caused by the overlapping of the two successive generated bubbles. Hence, the pressure is decreased in each expanding direction so the bubble cannot penetrate through the film in the perpendicular direction neither enlarge the crater diameter in the horizontal direction. With pitch distance of 60 $\mu \mathrm{m}$ and $30 \mu \mathrm{m}$ (Figs. 6.19c and 6.19d), the overlapping effect becomes more evident. The shallow groove formed on the film-glass interface implies the overlapping of the bubbles, which finally forms the tunnel in the donor.

This interaction effect can be also understood as a reduction of the effective energy used for moving the paste, since part of the gas pressure is used to forward transfer the paste, and part escapes through the drought channel formed.

\subsection{Comparison of ns and ps LIFT}

In this thesis study, two different diode-pumped solid state laser sources have been used in the present work: a ns-pulsed laser (Spectra Physics Explorer) and a high-power industrial ps-laser (EKSPLA Atlantic). Table 6.5 summarizes the main parameters of both lasers.

From this study it has been shown that both ns- and ps-lasers are suitable for printing lines of high viscosity silver paste. The transfer process has been parameterized by means of printing single paste voxels. It has been observed that the minimum energy required 
to transfer the paste increases with the thickness of the paste. The paste thickness and the gap are identified as key variables in the transfer of high viscosity pastes. Gaps with lengths similar or smaller that the paste thickness are required for forming a paste column between donor and acceptor substrate and leading to concrete transfer.

\begin{tabular}{|c|c|c|}
\hline Laser Source & $\begin{array}{c}\text { Spectra Physics } \\
\text { Explorer }\end{array}$ & $\begin{array}{l}\text { EKSPLA } \\
\text { Atlantic }\end{array}$ \\
\hline Wavelength (nm) & 532 & 532 \\
\hline Pulse duration & $<15 \mathrm{~ns}$ & $<13 \mathrm{ps}$ \\
\hline Power (W) & $>2$ & $>35$ \\
\hline Repetition rate (kHz) & $20-150$ & $400-1000$ \\
\hline Max. pulse energy ( لر) & 70 & 85 \\
\hline Pulse energy stability & $<3$ & $<2.2 \%$ \\
\hline$M^{2}$ & $<1.3$ & $<1.3$ \\
\hline Focusing optics & Optical scanner & Fixed lens \\
\hline Pulse energy ( $\mu \mathrm{J})$ & 14.4 & 10.6 \\
\hline Fluence $\left(\mathrm{J} / \mathrm{cm}^{2}\right)$ & 9.2 & 12.0 \\
\hline Paste thickness ( $\mu \mathrm{m})$ & 80 & 40 \\
\hline Gap distance $(\mu \mathrm{m})$ & 50 & 50 \\
\hline Scanning speed (m/s) & 2 & 0.06 \\
\hline Line height $(\mu \mathrm{m})$ & 55 & 35 \\
\hline Line width $(\mu \mathrm{m})$ & $90-150$ & 130 \\
\hline Aspect ratio & $0.36-0.61$ & 0.25 \\
\hline $\begin{array}{l}\text { Voxel transferred per } \\
\text { laser pulse }(p L)\end{array}$ & $495-825$ & 430 \\
\hline
\end{tabular}

Table 6.5: Comparison of ns- and ps- LIFT process parameters and line results.

Figure 6.20 shows false-color confocal images and profiles of LIFT lines printed with the optimum experimental parameters and the ns-laser (Fig. 6.20a) and the ps-laser (Fig. 
6.20b). Fig. 6.20c shows the transversal profiles of both lines. Table II summarizes the main experimental parameters used and the resulting morphological characterization.

Typical height of an optimum voxel is around $10 \mu \mathrm{m}$ while the height of lines is several times larger, while the width only slightly increases. This effect is related to an accumulative effect in the amount of the transferred paste when overlapping. Lines printed using the ns-laser are higher and narrower than those printed with ps-laser. The aspect ratios obtained using LIFT of this silver paste are better than those obtain with the same paste and standard screen-printing techniques.

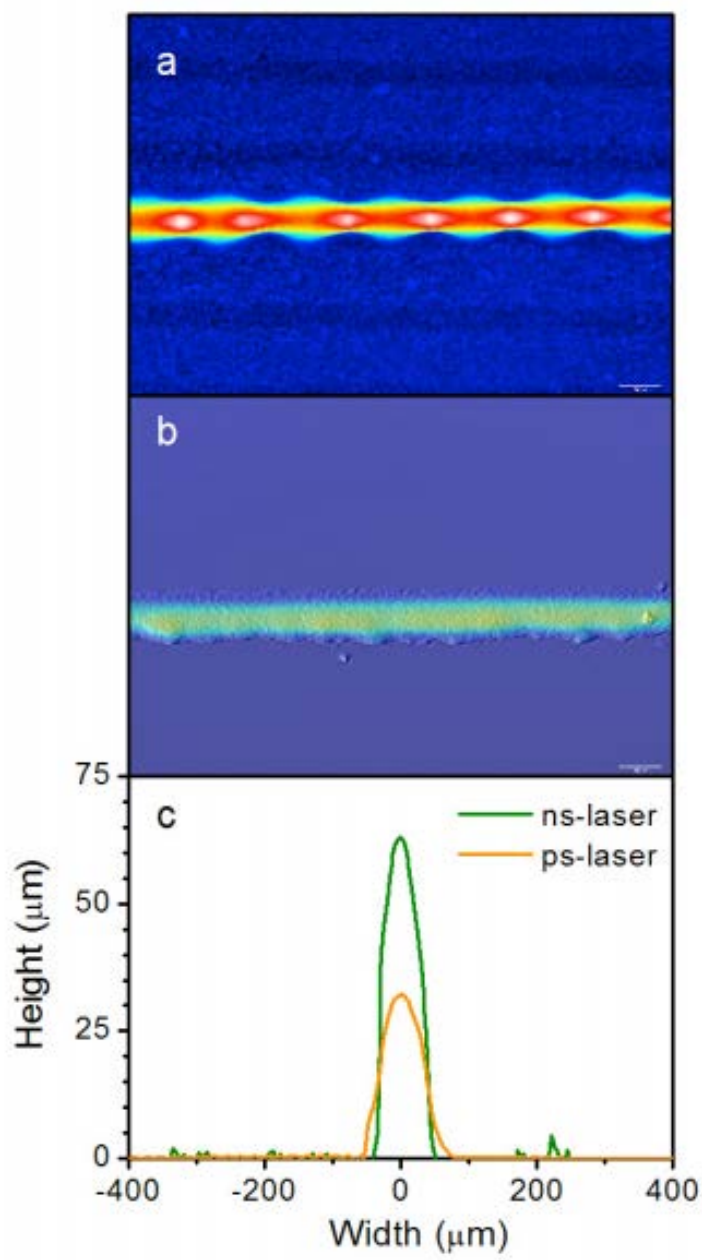

Figure 6.20: False-color confocal images of LIFT lines printed using (a) ns- and (b) ps-laser (c) cross-sectional profiles of the lines [86]. 


\section{Laser curing/sintering/melting of the silver paste}

The metallization process of the front contact of a solar cell consists of a pre-metallization process [27]. In this process, the metallic front electrodes are built with various techniques. However, in the case of printed silver paste, the metal particles surrounded by organic components give rise to a significant resistive performance, which increases the series resistance and affects the cell efficiency. Therefore, subsequent processes including a series of thermal treatment including evaporating the organic solvents (curing), compacting the metal particles (sintering) and etching the anti-reflective coating layer and electrically contacting the paste and the substrate (firing) [108], are needed for functionalizing the cell in the typical crystalline silicone solar cell fabrication process.

Laser sintering/melting is an additive manufacturing process, which utilizes laser beam irradiation scanning the powder bed layer by layer to create 3D structures. The materials involved in this method are normally metal or polymer powders. However, in the present study the silver paste is a mixture system consisting of metal powders, glass frits and organic components. Therefore, the term of curing of the silver paste involves evaporation of organic shell of the silver particles to allow the direct physical contact between particles. This process increases the conductance of the silver paste lines. The temperature for the curing process of silver paste is relatively low and mainly determined by the organic components of the paste. Whereas in the sintering process all organic component of the paste will be burned off and the silver particles must to bond with each other, resulting in a denser microstructure and significant enhancement of the conductance [109].

The temperature needed for sintering is higher than the temperature for the curing process, typically higher than $750^{\circ} \mathrm{C}$ for the micro-sized silver paste, but lower than the melting point of the silver particle (around $950^{\circ} \mathrm{C}$ ) (see Table 7.1). Nevertheless, the high sintering temperature may thermally damage some flexible substrate, such as CIGS thin film solar cell, which contains a series of thin film layers. 
One viable alternative to the conventional thermal treatments using furnace is to use laser. The pre-deposited line can be cured or sintered selectively by using continuous wave (CW) laser sources, without affecting the performance of the electronic device. Although the temperatures obtained can be high, the heating is local and most of the device is left unaffected by the laser. This is the reason why this process can be considered a low-temperature process. Laser curing have been studied in the case of nano-inks, nano-pastes and screen-printed micro-paste [110-113]. Compare to the furnace curing, the interaction time of silver paste and laser is short. In this work, it has been proved that with the optimal process parameters silver paste can be cured at high speeds.

\begin{tabular}{|l|l|l|l|}
\hline & Curing & Sintering & Melting \\
\hline Temperature $\left({ }^{\circ} \mathrm{C}\right)$ & $200-300$ & $750-950$ & 950 \\
\hline & evaporation of & Organic shell & Silver particles \\
& & burned off, silver & melted, a molten \\
& & particles bond \\
& & together, \\
& & microstructure get & \\
& & track forms & \\
\hline
\end{tabular}

Table 7.1: The different temperature and process effect in the curing, sintering and melting.

In order to develop a two-step, all-laser based, low-temperature process, laser curing/sintering process of silver paste transferred by LIFT was studied in this work. Different process parameters, such as laser power and scanning speed, were experimentally studied and the results obtained were discussed in terms of morphology of the line, adhesion of the track to the substrate after the curing process and enhancement of the electrical conductivity. 


\subsection{Experimental}

A Nd:YVO ${ }_{4} \mathrm{CW}$ laser (Spectra-Physics Millennia) with a $532 \mathrm{~nm}$ wavelength and output power up to $15 \mathrm{~W}$ was employed in the present work. Laser beams were scanned on top of DuPont PV17F silver paste printed lines using LIFT [13] by means of a galvanometer scanner (SCANLAB HurrySCAN II) (Fig. 7.1). The focused beam spot size was $35 \mu \mathrm{m}$. Sample morphology was analyzed using a Leica DCM3D confocal microscope and a scanning electron microscope (Hitachi S-3000N). The electrical response, namely the resistivity of 1-cm lines, was measured using a multimeter (Fluke 189 TRUE RMS).

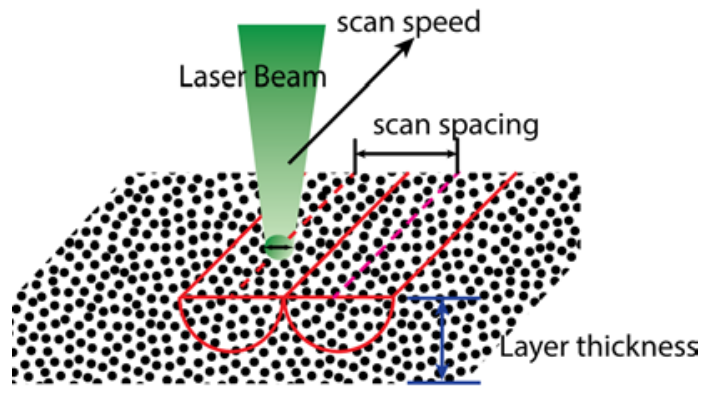

Figure 7.1: Schematic diagram showing sintering processing parameters.

In the first experiment, a total of 8 parallel passes along the line with a scan spacing of $20 \mu \mathrm{m}$ were made to assure the whole surface of the line was going to be treated. Laser power was varied from $2 \mathrm{~W}$ to $6 \mathrm{~W}$ and scanning speed from $4 \mathrm{~mm} / \mathrm{s}$ to $90 \mathrm{~mm} / \mathrm{s}$.

The second experiment was a two-step processes, which comprises a fast pre-heating process with laser power of $1.5 \mathrm{~W}$ and scan speed of $70 \mathrm{~mm} / \mathrm{s}$ and the second is a sintering process with laser power varied from $1.5 \mathrm{~W}$ to $10 \mathrm{~W}$ and scan speed of $10 \mathrm{~mm} / \mathrm{s}$. This experiment is applied on 11 lines with $1 \mathrm{~cm}$ of length.

\subsection{Results and discussions}

In order to compare the sintering processing condition, laser energy density is regarded as a key factor for evaluating the curing/sintering process results. 
In the present study, a CW laser is used and laser power and scan speed are selected as the variable parameters to investigate the process regime. Therefore, when the laser spot size and the scan spacing constant, the main parameter is the energy per unit length, which is defined as the ratio of laser average power and scan speed.

$$
Q_{s}=\frac{P}{v}
$$

Where $\mathrm{P}$ is the laser power $(\mathrm{W})$ and $v$ is the scan speed $(\mathrm{mm} / \mathrm{s})$.

Fig. 7.2 shows the microstructure of an as-deposited line, before any laser treatment. The silver grains can be clearly identified with spherically or near-spherically shaped particles. Table 1 presents the processing parameters in the experiments and the corresponding energy per unit length of each scan path.

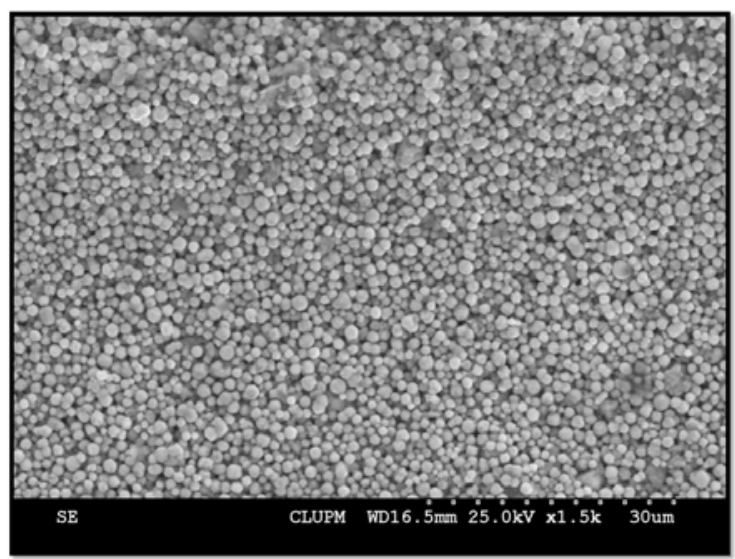

Figure 7.2: SEM image of the surface of as-deposited line silver paste without laser treatment.

The sintering experiment first starts with a fixed laser power of $2.7 \mathrm{~W}$ and the scan speeds were varied from $40 \mathrm{~mm} / \mathrm{s}$ to $90 \mathrm{~mm} / \mathrm{s}$. The geometry of the line does not change after the laser treatment, but the microstructure of the silver particles has been modified. 


\begin{tabular}{|c|c|c|c|c|}
\hline $\begin{array}{c}\text { Line } \\
\text { number }\end{array}$ & $\begin{array}{l}\text { P set } \\
\text { (W) }\end{array}$ & $\begin{array}{l}\text { Scan } \\
\text { speed } \\
\text { (mm/s) }\end{array}$ & $\begin{array}{c}\text { Energy per } \\
\text { unit } \\
\text { length }(\mathrm{J} / \mathrm{m})\end{array}$ & $\begin{array}{c}\text { Scan } \\
\text { Path } \\
\text { number }\end{array}$ \\
\hline 1 & 5.8 & 4 & 1450 & 1 \\
\hline 2 & 3.1 & 4 & 775 & 1 \\
\hline 3 & 4.7 & 40 & 118 & 1 \\
\hline 4 & 4.4 & 40 & 110 & 1 \\
\hline 5 & 3.9 & 40 & 98 & 1 \\
\hline 6 & 3.5 & 40 & 88 & 1 \\
\hline 7 & 3.1 & 40 & 78 & 1 \\
\hline 8 & 2.7 & 40 & 68 & 1 \\
\hline 9 & 2.7 & 50 & 54 & 1 \\
\hline 10 & 2.7 & 60 & 45 & 1 \\
\hline 11 & 2.7 & 70 & 38 & 1 \\
\hline 12 & 2.7 & 80 & 34 & 1 \\
\hline 13 & 2.7 & 90 & 30 & 1 \\
\hline 14 & 2.7 & 70 & 38 & 5 \\
\hline
\end{tabular}

Table 7.2: Process parameters for selective laser curing/sintering.

When the laser irradiates the line using scan speeds less than $90 \mathrm{~mm} / \mathrm{s}$, and energy per unit length above $30 \mathrm{~J} / \mathrm{m}$, not only curing and sintering but also a large region of melting took place. Molten structures of silver particles appear at the surface along the scan track where silver particles fused and agglomerate (Fig. 7.3). With one scan path, the laser tracks were sintered, while the morphology of the rest of the particles remained similar to the as-deposited line but should have been cured. This is due to the Gaussian distribution of the laser beam that the peak energy is centralized along the scan track while in the border the energy is lower and to the heat conduction to the surrounding 
area. It was found that the width of the molten track increases as the scan speed decreases (Fig. 7.4) as the heat has more time to travel to nearby areas.
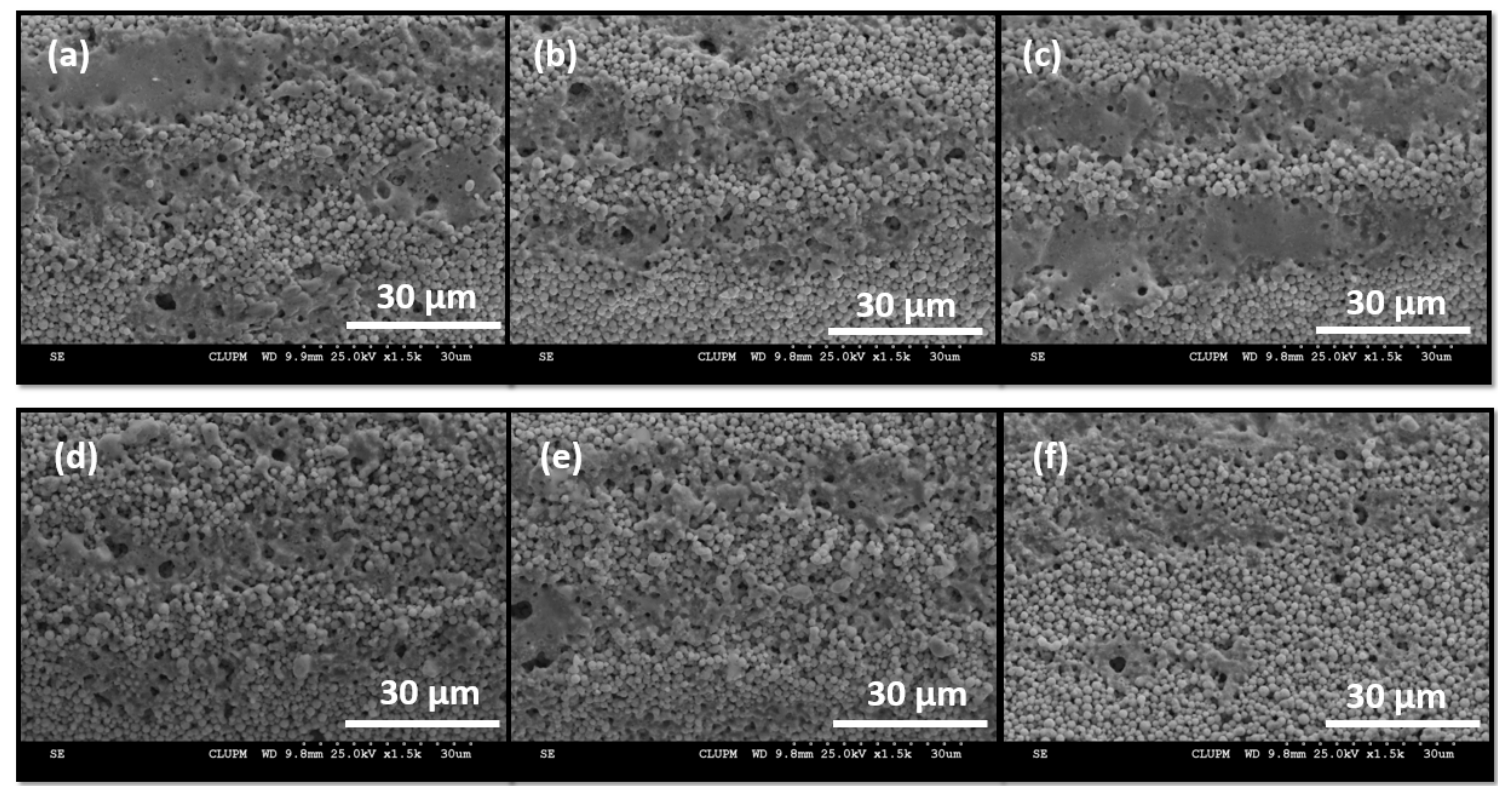

Figure 7.3: SEM images of the sintered region on silver paste surface with laser power of $2.7 \mathrm{~W}$ and varied speed: (a) $40 \mathrm{~mm} / \mathrm{s}$, (b) $50 \mathrm{~mm} / \mathrm{s}$, (c) $60 \mathrm{~mm} / \mathrm{s}$, (d) $70 \mathrm{~mm} / \mathrm{s}$, (e) 80 $\mathrm{mm} / \mathrm{s}$, (f) $90 \mathrm{~mm} / \mathrm{s}$.

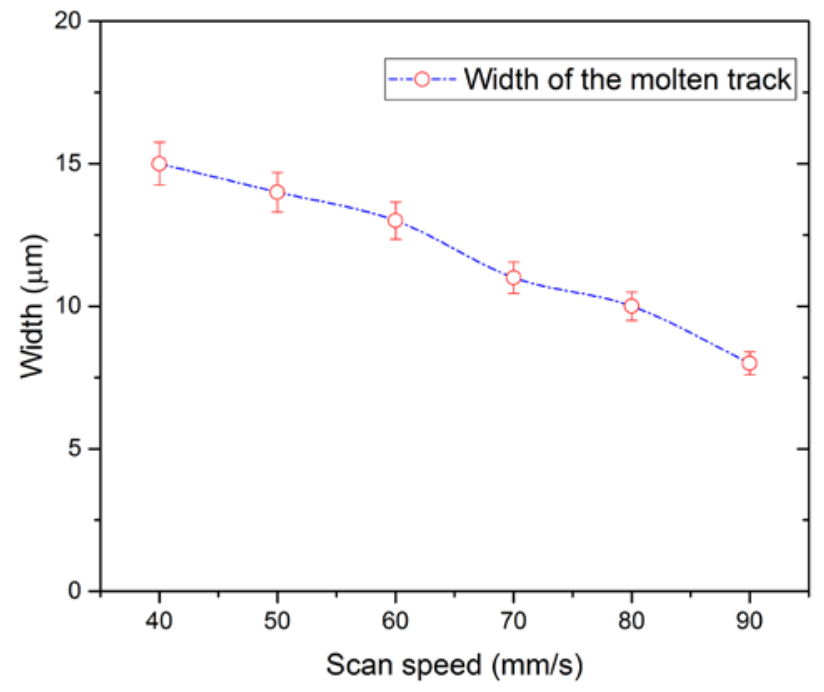

Figure 7.4: Width of the molten zone vs scan speed at laser power of $2.7 \mathrm{~W}$. 
With the laser beam moving on the surface of the silver paste, the received heat by the silver particles depends on the overlapping of laser spot. When increasing the scan speed, there is a reduction in the overlap area and the laser exposure time. Therefore, the energy input for the silver particles is reduced and the melt pool dimensions are also reduced. The particle size of the silver paste also affects the sintering process. The smaller particle size increases the driving force and makes the sintering process occur at a lower temperature [114].

Then laser irradiated tracks were produced by the scan speed of $40 \mathrm{~mm} / \mathrm{s}$ at different laser powers ranging from $2.7 \mathrm{~W}$ to $4.7 \mathrm{~W}$ to study the influence of the laser power in the sintering. Fig. 7.5 demonstrates the influence of the laser power in the sintering process of silver paste. It is observed that all the laser scanned tracks show a molten region as in the previous results. The increased power doesn't affect surface temperature of metal particles [115]. The width of the molten area increases as a function of laser power (Fig. 7.6). Moreover, the widths of these regions are wider than the ones obtained in the previous experiment. As seen in the Fig. 7.7, it is clear that the width of the molten region increases as a function of energy per unit length. With energy per unit length $Q_{s}>775 \mathrm{~J} / \mathrm{m}$, in the context of using a lower speed of $4 \mathrm{~mm} / \mathrm{s}$ and laser power of $3.1 \mathrm{~W}$ and $5.8 \mathrm{~W}$ respectively, it is observed a large area of liquefied silver particles agglomerated and consolidated (Fig. 7.8). The porosity is significantly decreased during this process. 

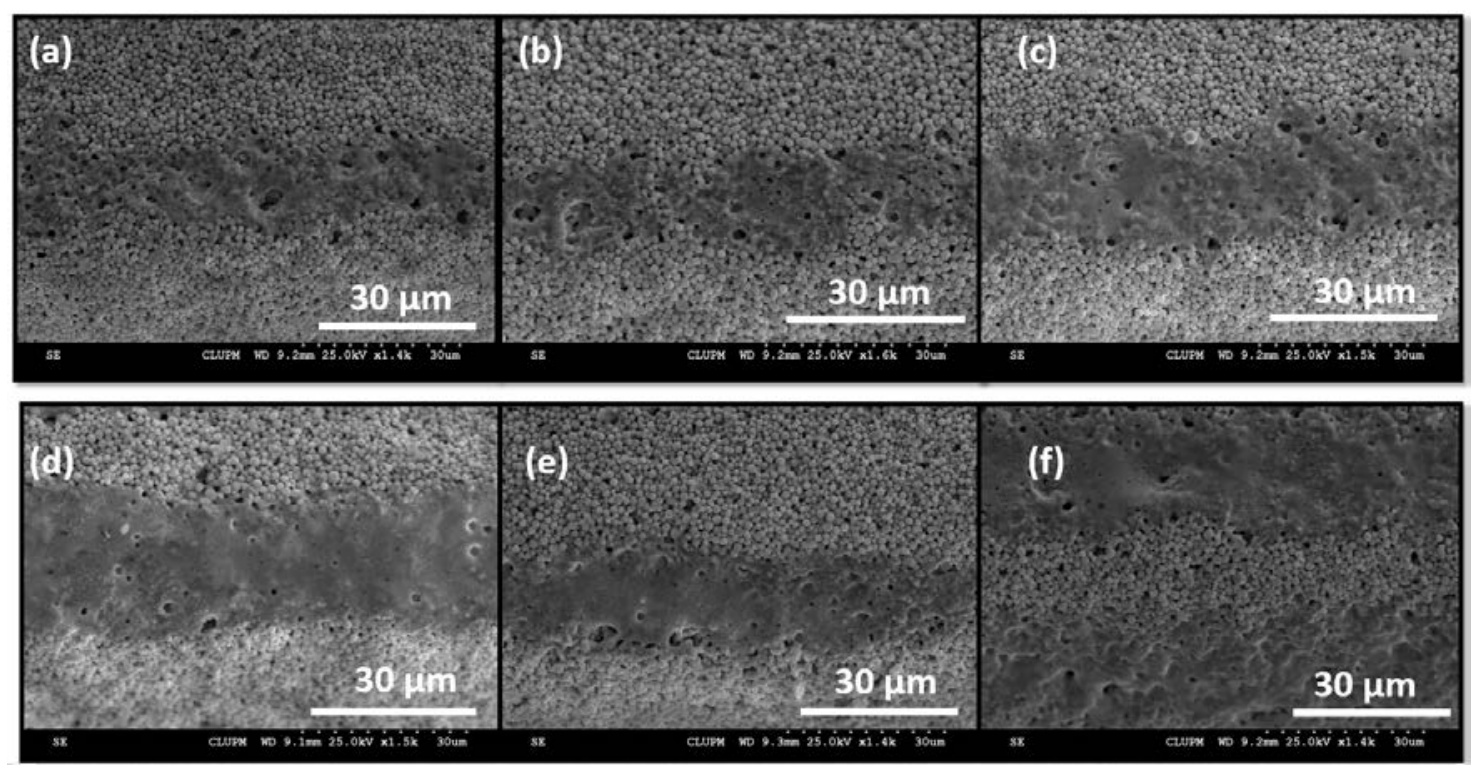

Figure 7.5: SEM images of the sintered region on silver paste surface with fixed scan speed of $40 \mathrm{~mm} / \mathrm{s}$ and varied laser power: (a) $2.7 \mathrm{~W}$, (b) $3.1 \mathrm{~W}$, (c) $3.5 \mathrm{~W}$, (d) $3.9 \mathrm{~W}$, (e) $4.4 \mathrm{~W}$, (f) $4.7 \mathrm{~W}$.

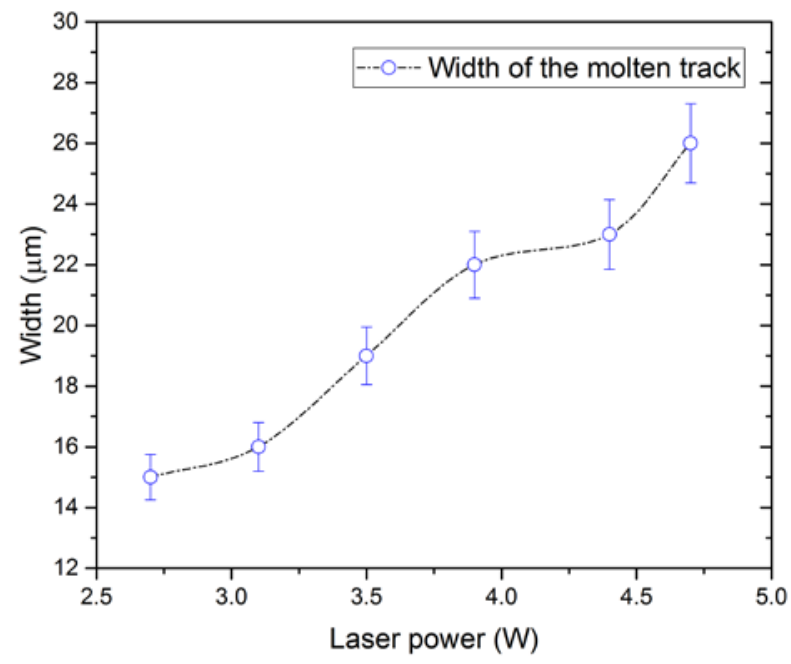

Figure 7.6: Influence of laser power on the width of the molten zone of silver particles at scan speed of $40 \mathrm{~mm} / \mathrm{s}$. 


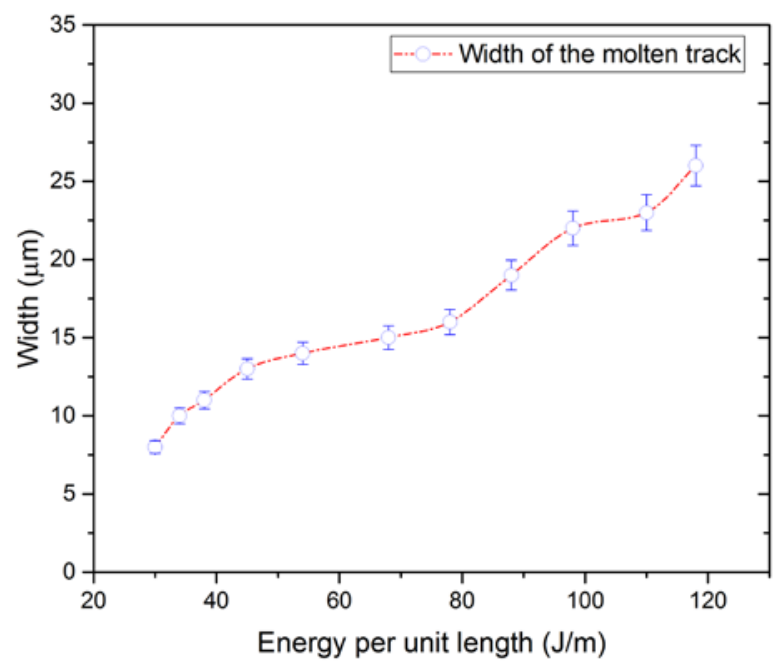

Figure 7.7: Influence of energy per unit length on the width of the molten zone of silver particles.

However, laser heating affects not only the microstructure but also the adherence of the line to the substrate. All the lines scanned with scan speed less than $70 \mathrm{~mm} / \mathrm{s}$, or laser energy per unit length above $38 \mathrm{~J} / \mathrm{m}$, peel off from the substrate and broke off.

Therefore, it is necessary to heat up the line with fast speed and low power, with energy per unit length $\mathrm{Q}_{\mathrm{s}}<38 \mathrm{~J} / \mathrm{m}$, in order to realize low temperatures thermal treatment which is similar to those reported for drying the paste in a furnace. Fig. 7.9 illustrates the effect of increasing numbers of scanning path. When scanning 5 paths with laser power of $2.7 \mathrm{~W}$ and scan speed of $40 \mathrm{~mm} / \mathrm{s}$, necking of the particles occurred significantly without liquefying. The observed microstructure indicates that the sintering take place with this process window.

Despite of detaching from the substrate, the line was sintered well since the resistivity decreases two orders of magnitude when compared to the as-deposited line, from $1170 \pm 50 \mu \Omega \cdot \mathrm{cm}$ to $26 \pm 5 \mu \Omega \cdot \mathrm{cm}$. 


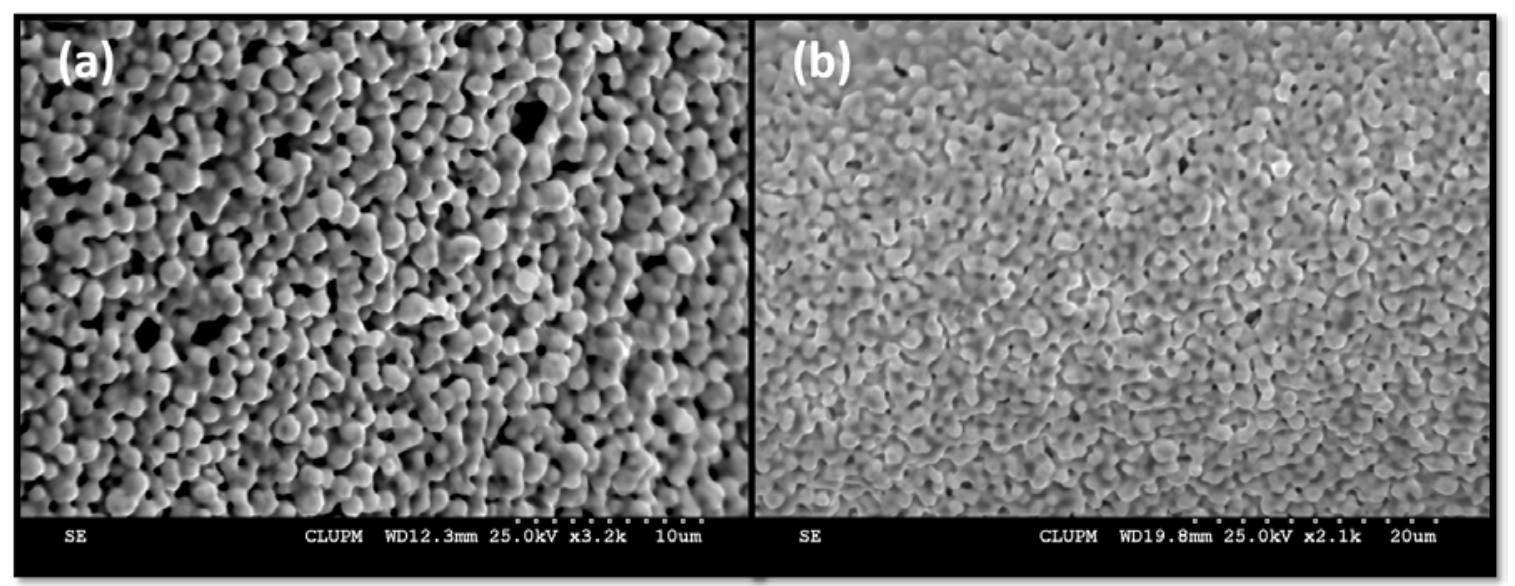

Figure 7.8: SEM images of the sintered region on silver paste surface with scan speed of $4 \mathrm{~mm} / \mathrm{s}$ and two different laser power: (a) 3.1W, (b) $5.8 \mathrm{~W}$.

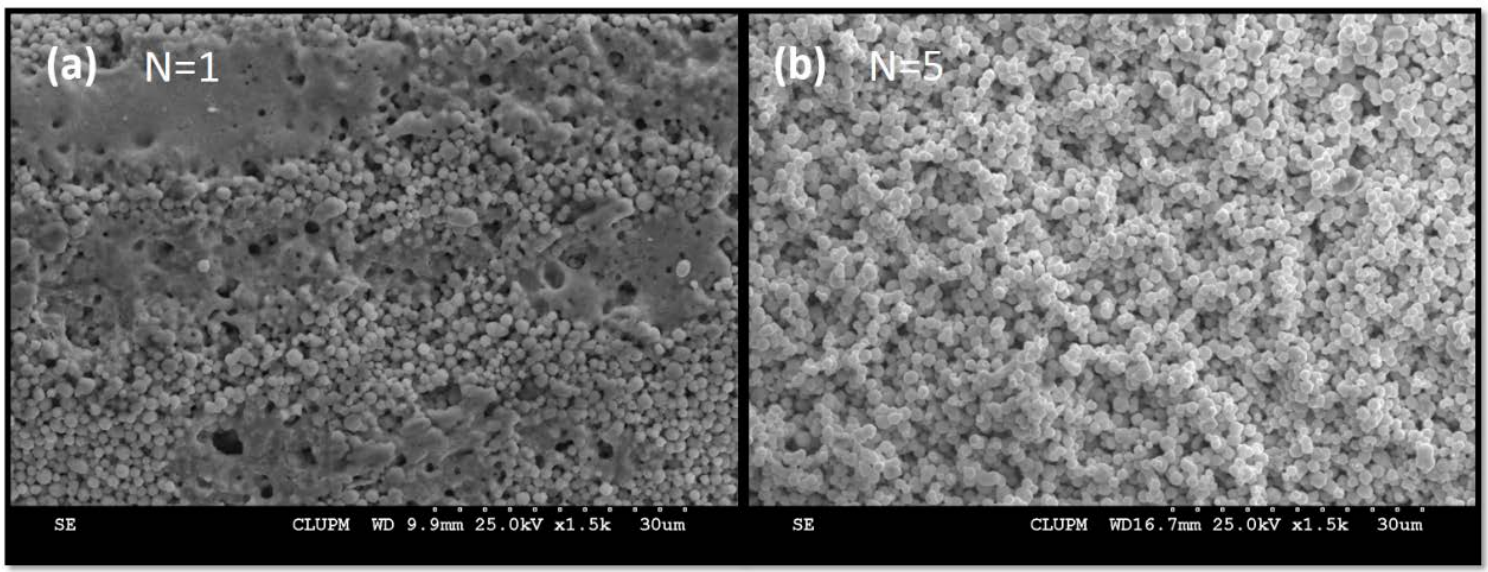

Figure 7.9: SEM images of the sintered region on silver paste surface with scan speed of $4 \mathrm{~mm} / \mathrm{s}$, laser power of $2.7 \mathrm{~W}$ and two scan path numbers: (a) $\mathrm{N}=1$, (b) $\mathrm{N}=5$. 


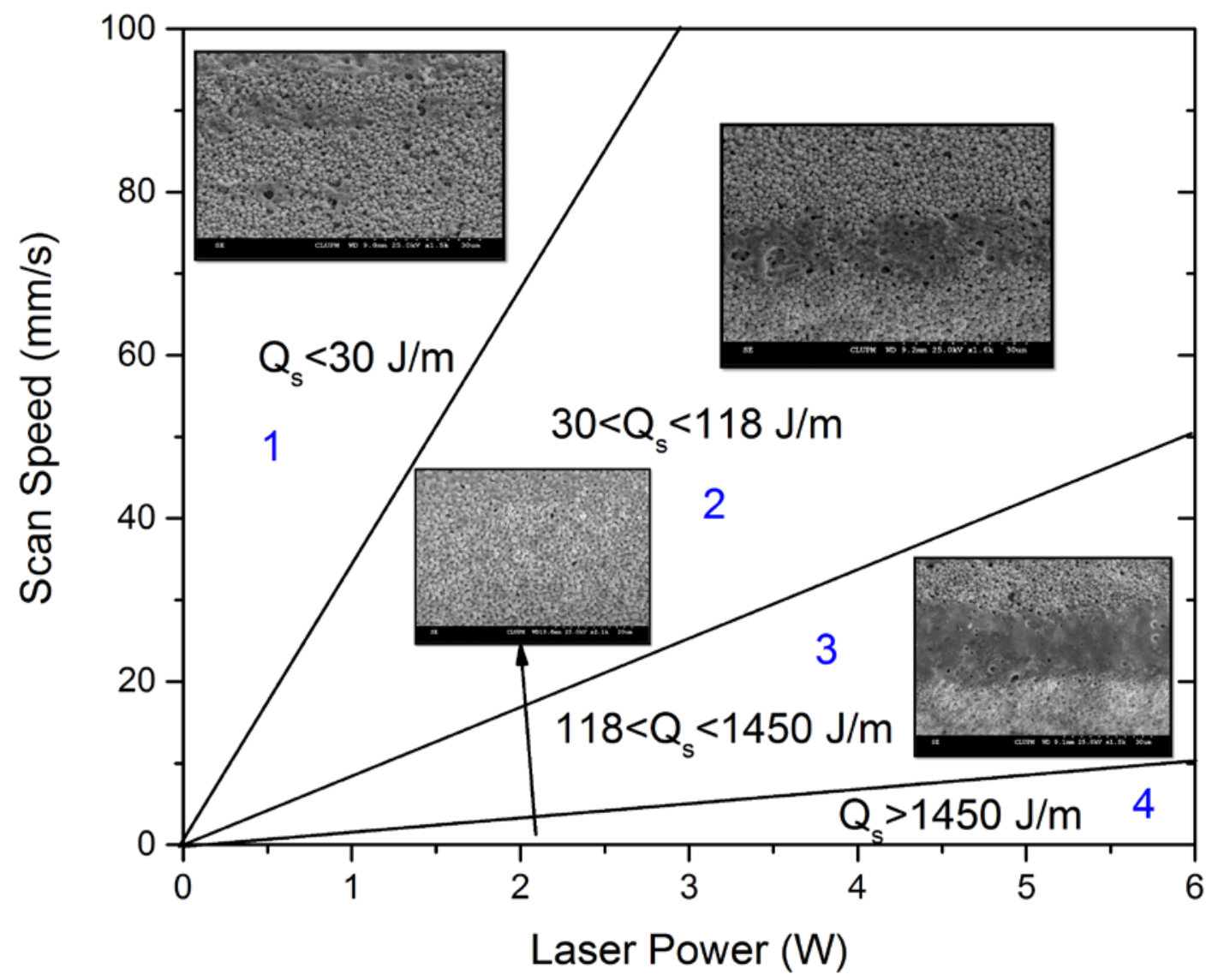

Figure 7.10: Processing window for single path scan on silver paste surface. Processing conditions are scan speed (4-90mm/s), laser power (2.7 - 5.8 W) and scan spacing of 20 $\mu \mathrm{m}$.

Fig. 7.10 summarizes the processing window and differences in the sintered surface of the different regions and shows characteristics morphology of the silver paste surface.

- With $\mathrm{Q}_{s}<30 \mathrm{~J} / \mathrm{m}$ (region 1), although there is no apparent melting in sight, the curing process can take place involving the evaporation of the organic solvent.

- When using energy per unit length $30<\mathrm{Q}_{\mathrm{s}}<118 \mathrm{~J} / \mathrm{m}$ (region 2), the silver particles are partial melted. Despite of the occurrence of a shallow single molten track, agglomeration of the particles can be observed beside the track. 
- With $118<\mathrm{Q}_{\mathrm{s}}<1450 \mathrm{~J} / \mathrm{m}$ (region 3), it is observed an enlarged molten track and coherently bonded particles at the boundary which is favorable for the laser sintering of silver paste. The completely molten track is considered to be an excessive heating effect.

- Finally, for $\mathrm{Q}_{\mathrm{s}}>1450 \mathrm{~J} / \mathrm{m}$ (region 4) the silver particles necked with each other and shows good consolidation which is similar to the sintered area beside the molten track in region 3.

With the knowledge of these results, a two-step laser curing/sintering experiment was performed to investigate the effect of the pre-heating. Fig. 7.11 summarizes the SEM images of the sintered region on the silver paste surface layer with the two-step laser treatment. Necking occurred with $Q_{s}=58 \mathrm{~J} / \mathrm{m}$, i.e. laser power of $0.6 \mathrm{~W}$ and scan speed of $10 \mathrm{~mm} / \mathrm{s}$ and molten silver particles appear on the surface layer. With increasing the laser power, the molten area increases which is in agreement with the previous observation.

Moreover, energy per unit length of first pre-heating scan path is only $8.3 \mathrm{~J} / \mathrm{m}$, which is smaller than the threshold energy for peeling off the line. However, with assistance of this pre-heating process, the lines didn't detach from the substrate although the second path with irradiation of energy per unit length above $38 \mathrm{~J} / \mathrm{m}$ and reaching up to $392 \mathrm{~J} / \mathrm{m}$. This enabled the measurement of the resistivity of the lines. Table 7.3 represents the electrical performance of the two-step laser curing/sintering lines. R1 is the average resistance before the laser treatment and $\mathrm{R} 2$ is the value after treatment. $r$ is the resistivity of the line after laser treatment. A corresponds to the average cross-sectional area of each line.

Several laser tracks are experimentally needed to cure the whole line. The temperature distribution obtained with the different laser tracks is considered to be independent of previous tracks, because of the small temperature increasing, the fast cooling due to heat diffusion and the elapsed time between two tracks in the same section. 
The largest scanning speeds lead to an exposure time in the order of several tens of ms. Experimental results show that large exposure time allows the silver grains to melt and sinter, but with extremely bad adherence to the substrate performance, since the line peel off when it is manipulated.

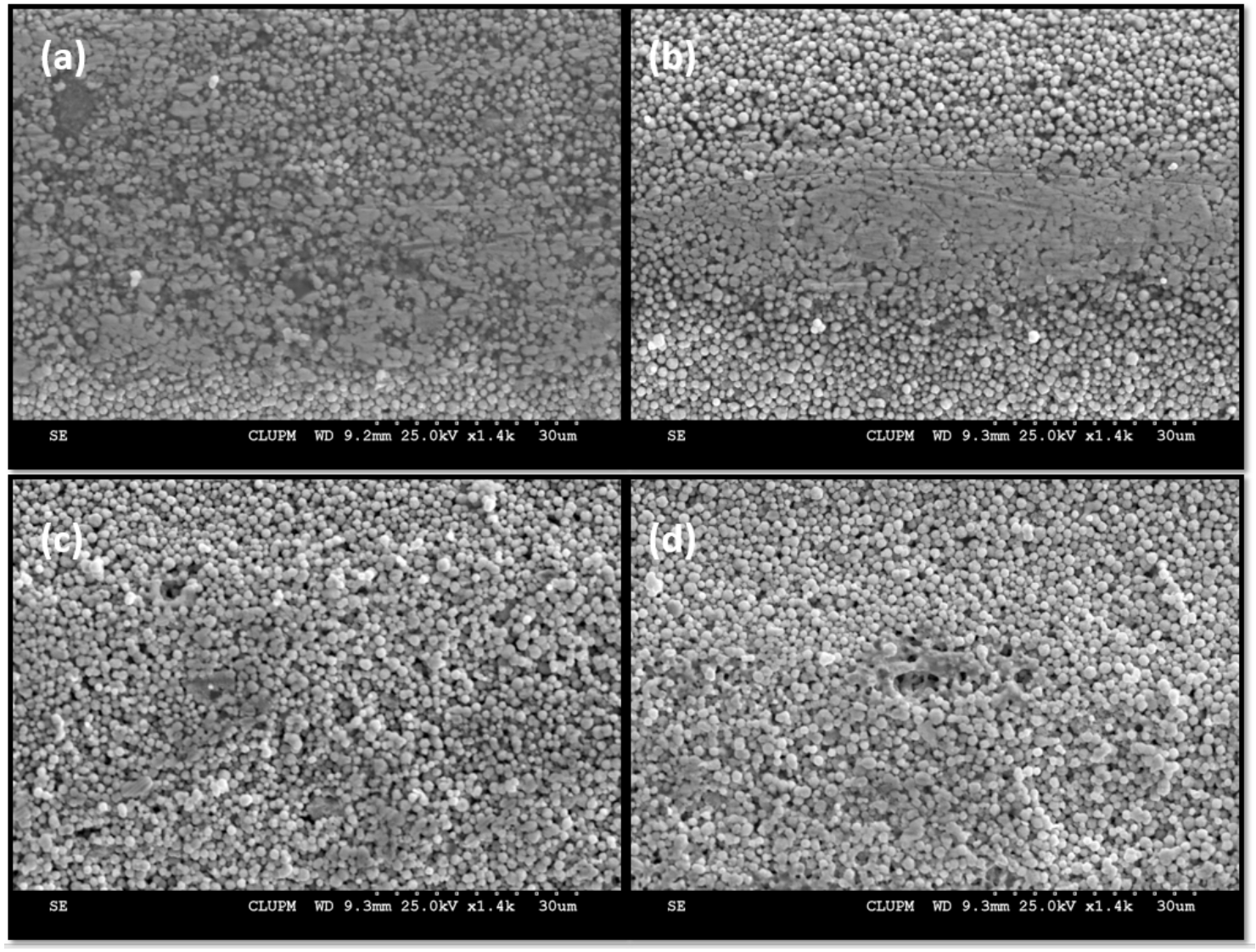

Figure 7.11: SEM images of the sintered region on silver paste surface with two-step treatment, first preheating with scan speed of $70 \mathrm{~mm} / \mathrm{s}$, laser power of $1.5 \mathrm{~W}$; second scan path with scan speed of $10 \mathrm{~mm} / \mathrm{s}$ and different laser power: (a) $3.1 \mathrm{~W}$, (b) $1.9 \mathrm{~W}$, (c) 1.2 W, (d) $0.6 \mathrm{~W}$.

Several lines have been treated completely by overlapping different laser tracks. The temperature distribution obtained with the different laser tracks is considered to be independent of previous tracks, because of the small temperature increasing, the fast cooling due to heat diffusion and the elapsed time between two tracks in the same section. 
The largest scanning speeds lead to an exposure time in the order of several tens of ms. Experimental results show that large exposure time allows the silver grains to melt and sinter, but with extremely bad adherence to the substrate performance, since the line peel off when it is manipulated.

\begin{tabular}{ccccccccc}
\hline $\begin{array}{c}\text { Line } \\
\text { number }\end{array}$ & Power & $\begin{array}{c}\text { Energy } \\
\text { per unit } \\
\text { length of } \\
\text { first } \\
\text { path(J/m) }\end{array}$ & $\begin{array}{c}\text { Energy } \\
\text { per unit } \\
\text { length of } \\
\text { second } \\
\text { path(J/m) }\end{array}$ & $\mathbf{R 1}(\Omega)$ & $\mathbf{R 2}(\Omega)$ & $\Delta \mathbf{R}$ & $\begin{array}{c}\mathbf{A} \\
\left(\boldsymbol{\mu} \mathbf{m}^{\mathbf{2}}\right.\end{array}$ & $\begin{array}{c}\mathbf{r} \\
(\boldsymbol{\mu} \Omega \cdot \mathbf{c m})\end{array}$ \\
\hline $\mathbf{1}$ & 0.6 & 8.3 & 58 & 6.4 & 2.0 & 4.4 & 5902 & 118.0 \\
\hline $\mathbf{2}$ & 0.8 & 8.3 & 78 & 5.8 & 1.1 & 4.7 & 4925 & 54.2 \\
\hline $\mathbf{3}$ & 1.0 & 8.3 & 98 & 5.2 & 1.0 & 4.2 & 5011 & 50.1 \\
\hline $\mathbf{4}$ & 1.2 & 8.3 & 118 & 4 & 1.0 & 3 & 6447 & 64.5 \\
\hline $\mathbf{5}$ & 1.4 & 8.3 & 137 & 5.5 & 1.0 & 4.5 & 6088 & 60.9 \\
\hline $\mathbf{6}$ & 1.6 & 8.3 & 157 & 6.6 & 1.0 & 5.6 & 6453 & 64.5 \\
\hline $\mathbf{7}$ & 2.0 & 8.3 & 196 & 4.3 & 0.7 & 3.6 & 8242 & 57.7 \\
\hline $\mathbf{8}$ & 2.4 & 8.3 & 235 & 5.5 & 0.6 & 4.9 & 7557 & 45.3 \\
\hline $\mathbf{9}$ & 2.8 & 8.3 & 274 & 2.9 & 0.9 & 2 & 7625 & 68.6 \\
\hline $\mathbf{1 0}$ & 3.1 & 8.3 & 314 & 7 & 0.4 & 6.6 & 7689 & 30.8 \\
\hline $\mathbf{1 1}$ & 3.9 & 8.3 & 392 & 6 & 0.3 & 5.7 & 8032 & 24.1 \\
\hline
\end{tabular}

Table 7.3: The electrical properties of the laser treated lines.

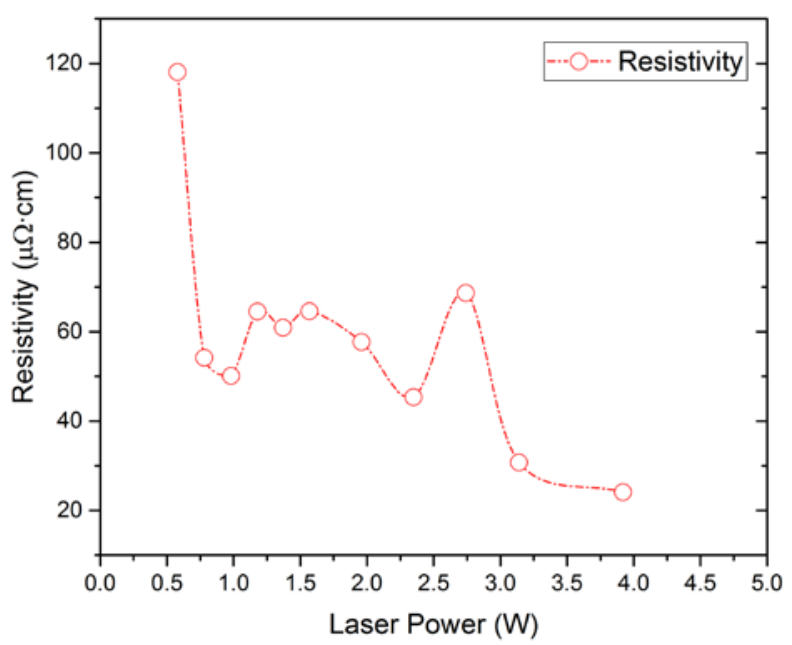

Figure 7.12: The bulk resistivity of finger line as a function of laser power with two-step sintering. 
SOA Furnace Laser

$\begin{array}{llll}\text { Resistivity }(\mu \Omega \cdot \mathrm{cm}) & 30 & 40 & 24\end{array}$

Table 7.4: The resistivity of the silver paste lines sintered using different method.

From Fig. 7.12, it is concluded that the bulk resistivity of the finger lines decreases as a function of laser power, which indicates that the larger laser power makes more evaporation of the organic components and strongly necking the silver particles. And Table 7.4 shows the resistivity of the silver paste lines treated using different method and the state of art (SOA) result. It can be seen that with laser curing/sintering treatment, the line obtains the best sintering results.

Laser curing/sintering has been shown that can be used as a low temperature process due to its capability to produce local heat suitable for the process but leaving the rest of the cell almost at room temperature.

In this chapter, it is proved that laser curing and sintering of silver paste lines printed using LIFT can be achieved at different laser powers and scanning speeds, but there is a small parametric window for obtaining the desired properties (increased electrical conductivity and good mechanical adhesion), making it a very sensitive process.

It has been observed that a pre-heating process at fast scanning speed and with low laser power, which can be also regarded as a curing process, is needed to reduce the damage to the silver line and to have the best adhesion results. After that pre-heating process the printed lines can be irradiated even at higher values of energy per length and more scanning paths can be applied to neck all the silver particles to obtain the best sintering performance without compromising the adhesion of the line. In that case, the electrical resistivity has been decreased by two orders of magnitude with a good morphology after laser sintering. 
The geometry of the line is not affected by the laser curing and sintering when the lines remain on the substrate. This preserves the aspect ratio obtained when printing lines using LIFT. Therefore, it is proved that the selective laser curing and sintering can be combined with other laser direct writing printing techniques and be implemented in future photovoltaic industrial applications for developing an all-laser based metallization process. 


\section{Proof of concept in a final device}

In chapter 4, we have discussed the influence of the acceptor on the morphology of the printed line. In the CIGS substrate surface, it is hardly to obtain a well-defined line by using the original silver paste. And there is almost no transfer when using a near-threshold energy. On the other hand, the morphology of the line is sacrificed with continuity when using high energy range (Fig. 8.1). If the laser spot is defocused, some part of the line can be transferred with a good morphology but losing continuity (Fig. 8.2). Nevertheless, with adding a certain quantity of thinner, the morphology of the line will be improved.
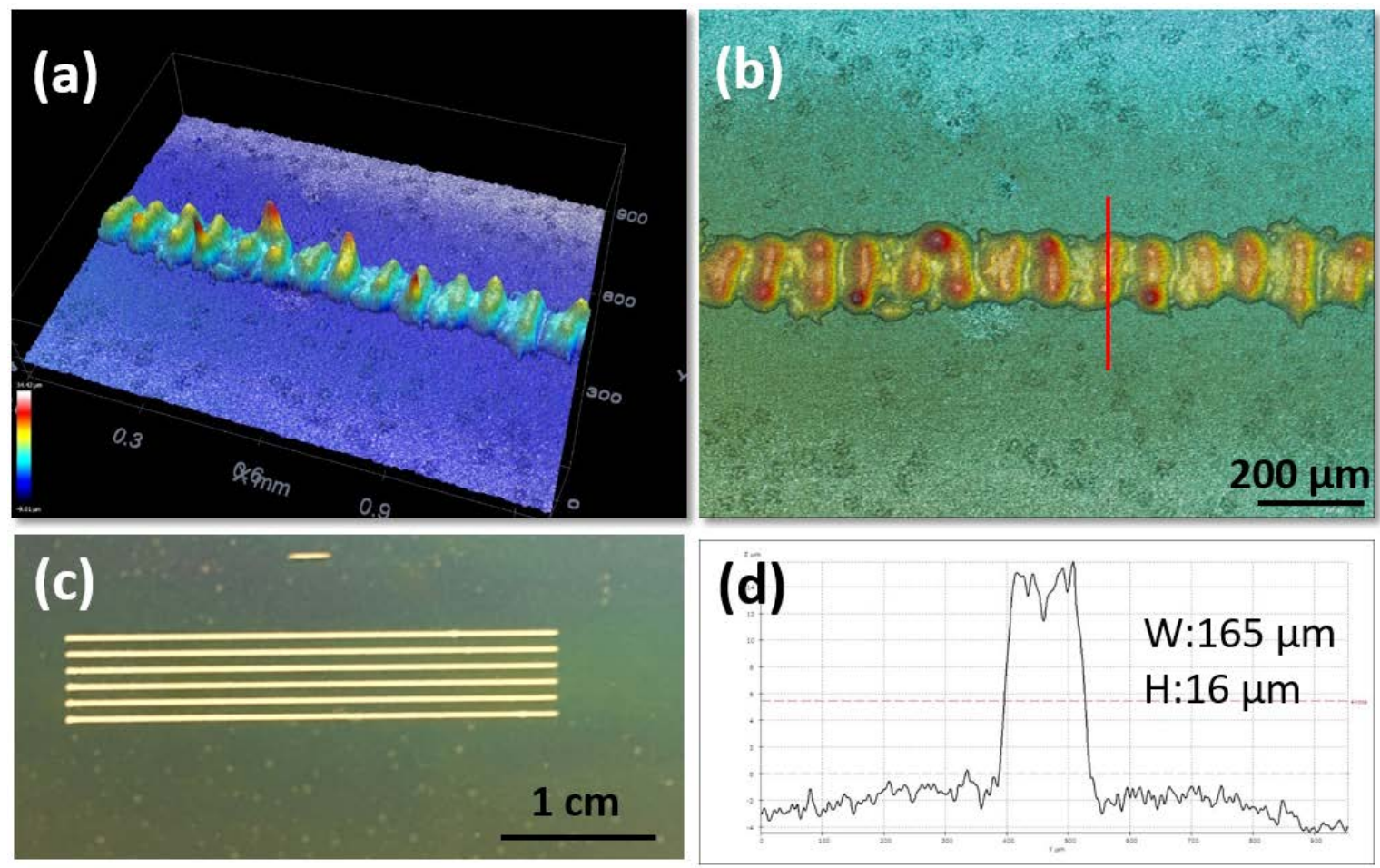

Figure 8.1: The confocal images of LIFT line shows good continuity but bad morphology with process parameters: pulse energy $(11 \mu \mathrm{J})$, scan speed $(1800 \mathrm{~mm} / \mathrm{s})$, laser frequency (20 kHz), film thickness (55 $\mu \mathrm{m})$. 


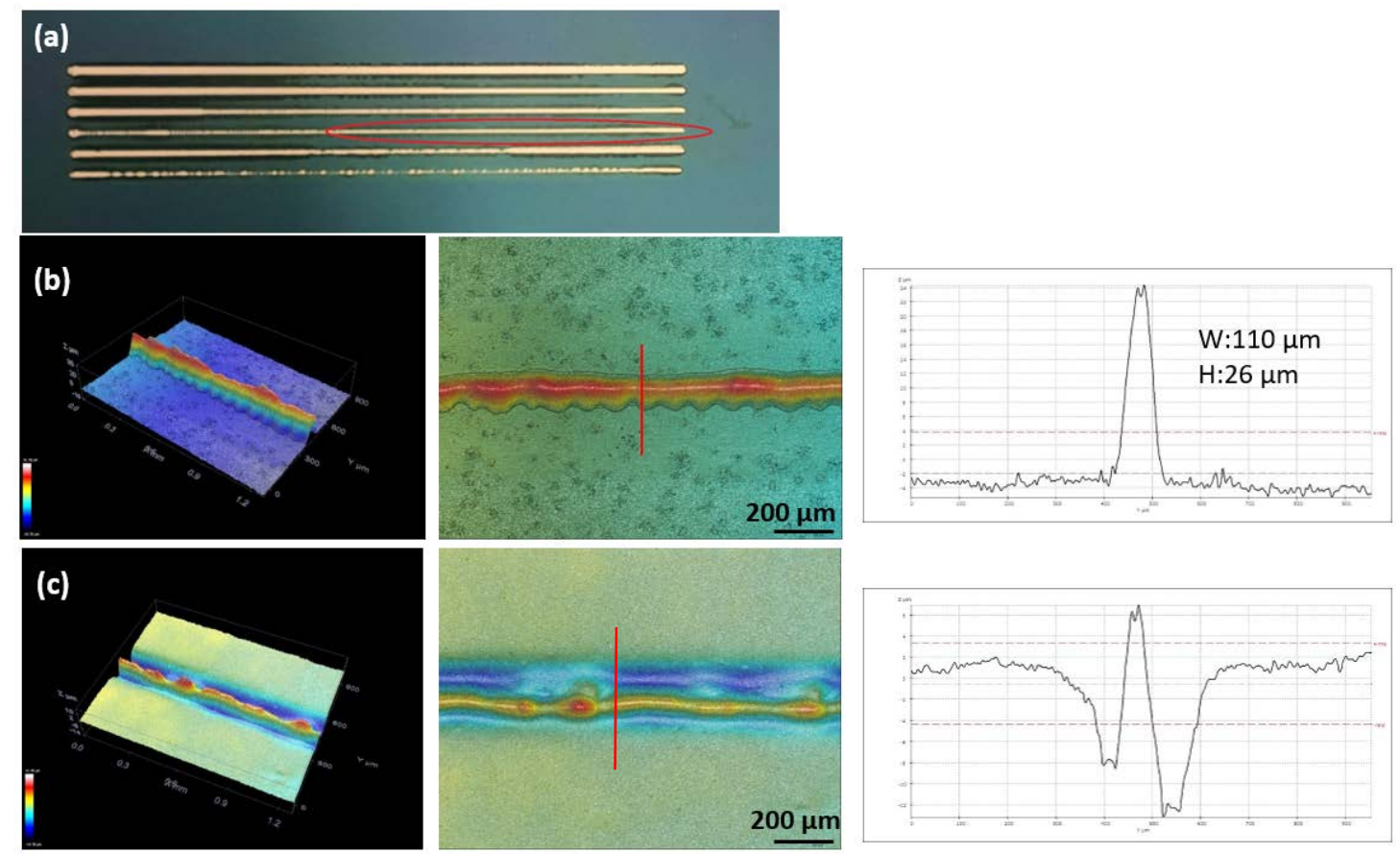

Figure 8.2: The confocal images of LIFT line shows good morphology but bad continuity with process parameters: pulse energy $(11 \mu \mathrm{J})$, scan speed $(1800 \mathrm{~mm} / \mathrm{s})$, laser frequency (20 kHz), film thickness (55 $\mu \mathrm{m})$; (a) The line deposited by LIFT on the CIGS substrate, (b) Morphology of the printed line, (c) The morphology of the groove left in the donor.

\subsection{LIFT line with thinner on CIGS substrate}

In order to find an intermediate performance between line continuity and morphology, $4.2 \mathrm{wt} \%$ of thinner is added for changing the fluid properties reducing viscosity, yield stress and surface tension of the silver paste. The silver paste gets more fluid and easy to wet the CIGS surface. The best morphology of the line (Fig. 8.3) was obtained with the following process parameters: pulse energy (11 $\mu)$, scan speed $(1800 \mathrm{~mm} / \mathrm{s})$, laser frequency $(20 \mathrm{kHz})$, film thickness $(55 \mu \mathrm{m})$. 

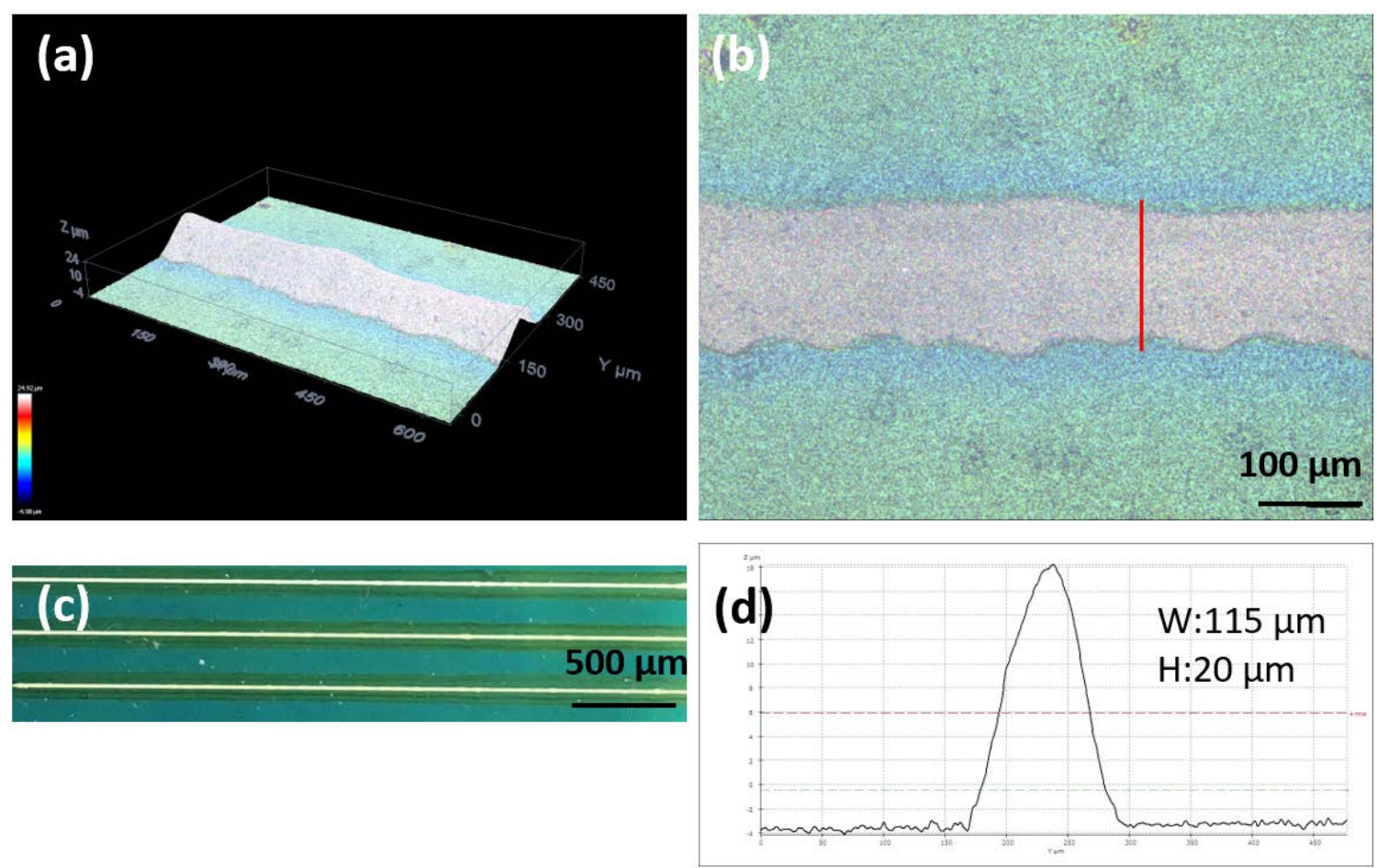

Figure 8.3: The confocal images of LIFT line obtained by salver paste with $4.2 \mathrm{wt} \%$ of thinner. Process parameters: pulse energy $(11 \mu \mathrm{J})$, scan speed $(1800 \mathrm{~mm} / \mathrm{s})$, laser frequency $(20 \mathrm{kHz})$, film thickness $(55 \mu \mathrm{m})$; (a) Topography of the printed line in 3D vision, (b) Microscope image of the printed line, (c) The line deposited by LIFT on the CIGS substrate showing continuity, (d) The transverse profile of the line.

Fig. 8.4 illustrates the transverse profiles of the line deposited by ns-pulsed green laser with optimal process parameters respectively on textured c-Si and CIGS substrates. It is clearly observed that the line deposited on textured c-Si has larger height and smaller width than the one printed on CIGS substrate. These results are in agreement with the voxel printing using single laser pulse. 


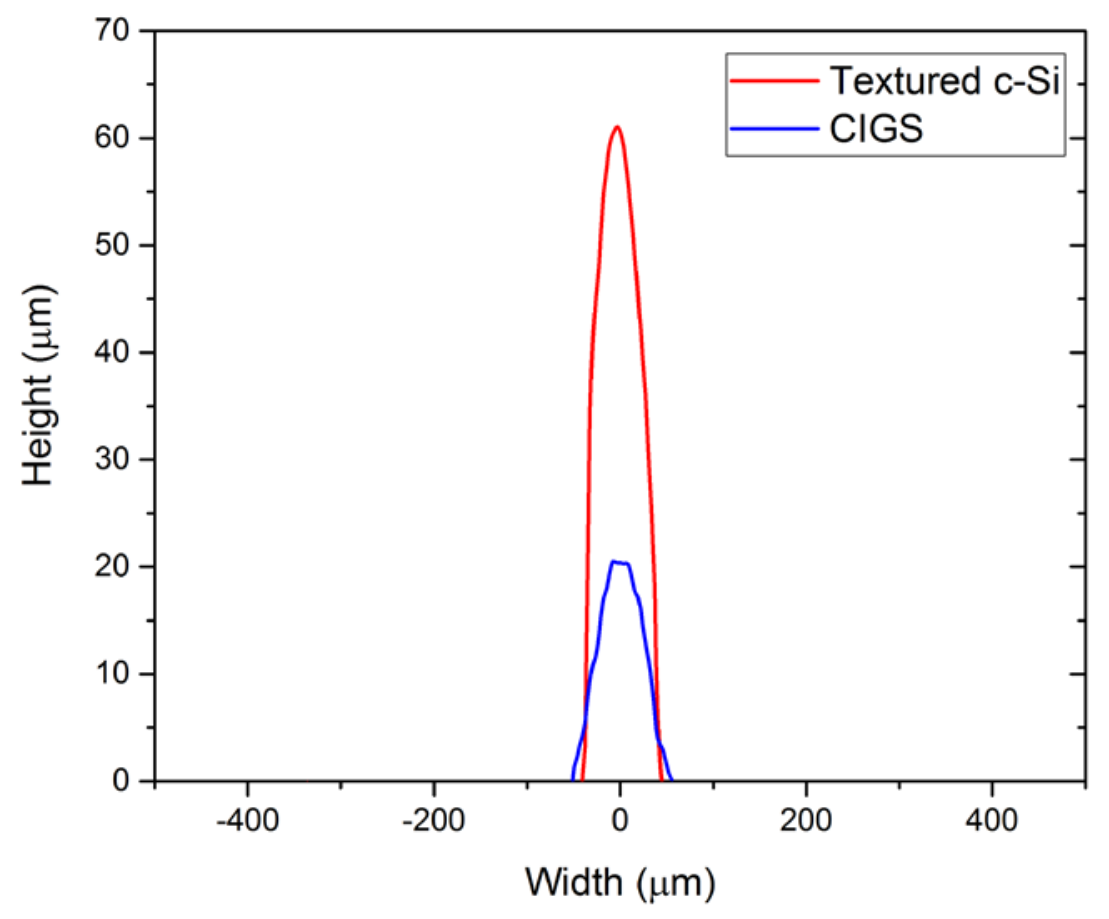

Figure 8.4: The transverse profiles of LIFT line obtained with optimal process parameters of the ns-pulse green laser on both textured c-Si and CIGS substrates.

\subsection{Mechanical properties of printed lines}

The mechanical behaviour of the printed lines has been characterized by means of studying the flexibility of the lines. The test cell is wrapped against several cylinders with different radius (Fig. 8.5): Metallic lines are adjusted then to a sector of a coaxial circumference with the cylindrical axis. After being bended, a visual inspection has performed to study the adhesion of the line. The minimum bending radius that can withstand the lines before peeling off determine the flexibility of the sample. Bending cylinders with radius $55 \mathrm{~mm}, 45 \mathrm{~mm}, 35 \mathrm{~mm}, 25 \mathrm{~mm}, 20 \mathrm{~mm}, 3 / 4$ " (19,05 mm), and 1/2" $(12.7 \mathrm{~mm}$ ) have been used. Lines do withstand bending down to the minimum radius, without peeling off. 

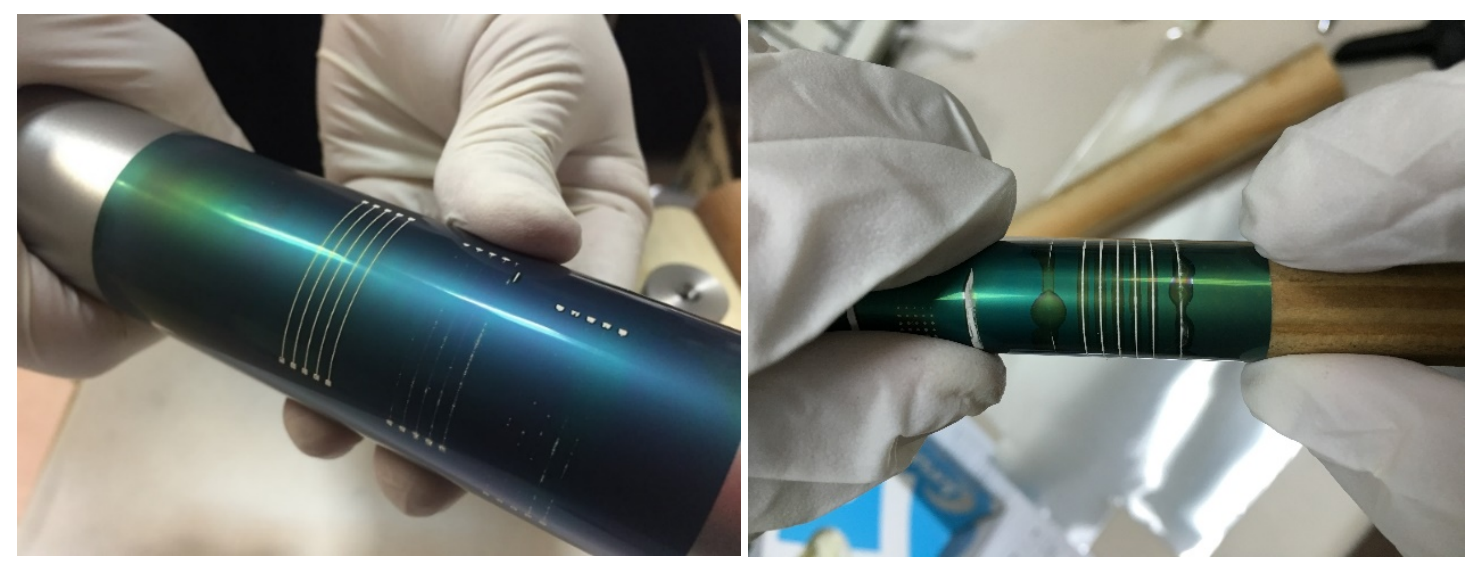

Figure 8.5: LIFT printed lines bended using a test cylinder with radius $3 / 4 "$.

\subsection{Metallization of solar cells}

To verify the concept of the two-step laser-based metallization process for solar cell, a simple design of front contact is carried out with three fingers and a $1 \times 1 \mathrm{~cm}^{2}$ pad connecting the end of the fingers for collecting current (Fig. 8.6). The pattern was then printed by LIFT process with the nanosecond green laser with the optimal process parameters obtained. In the present work, a $4.2 \mathrm{wt} \%$ of thinner was added to the silver paste for obtaining a continuous line with good morphology. The process parameters are listed in the Table. 8.1.

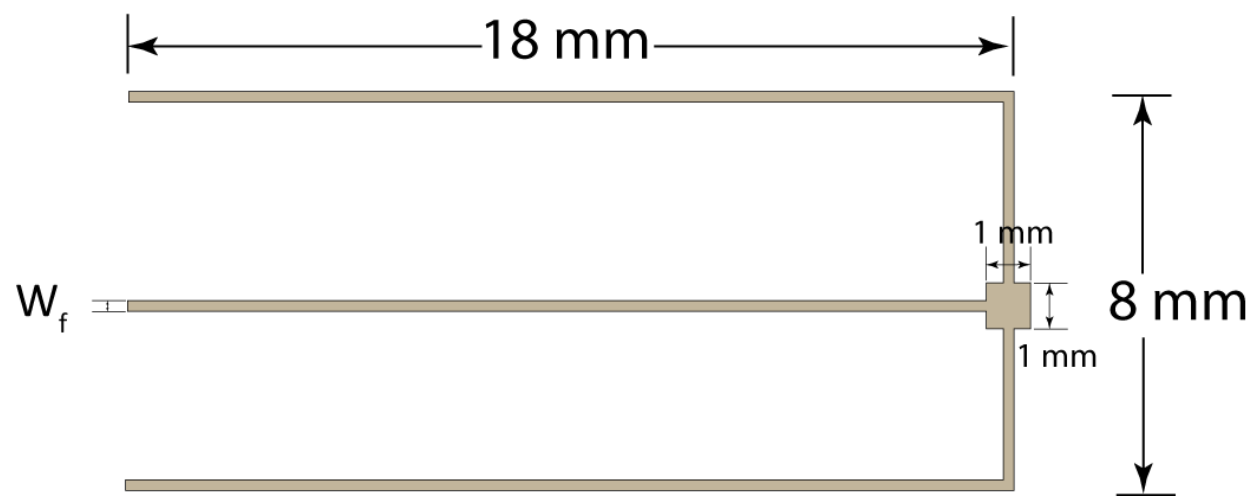

Figure 8.6: Schematic of front contact design in the final device. 


\begin{tabular}{cc}
\hline Processing Parameters & Values \\
\hline Laser frequency & $20 \mathrm{KHz}$ \\
\hline Scan speed & $2 \mathrm{~m} / \mathrm{s}$ \\
\hline Focus position & On Focus \\
\hline Paste thickness & $55 \pm 5 \mu \mathrm{m}$ \\
\hline GAP & $50 \mu \mathrm{m}$ \\
\hline Thinner & $4.2 \mathrm{wt} \%$ \\
\hline Spot size & $26 \mu \mathrm{m}$ \\
\hline Acceptor substrate & CIGS solar cell with TCO on the top layer \\
\hline Pulse energy & $11 \mu \mathrm{J}$ \\
\hline Geometry of LIFT line & $150-160 \mu \mathrm{m}$ \\
\hline width & $23-26 \mu \mathrm{m}$ \\
\hline height
\end{tabular}

Table 8.1: Process parameters and geometry of the front contacts printed by LIFT on CIGS solar cell.

Once the front contact pattern printed on CIGS cell by LIFT, the two sample (1 and 2) solar cells was cured in the furnace at $200^{\circ} \mathrm{C}$ for 1 hour. Then the pattern is isolated by laser for the electrical test (see Fig. 8.7). The solar cell characterization was performed with a mask of $2.1 \mathrm{~cm}^{2}$ with $2.1 \mathrm{~cm}$ in length and $1 \mathrm{~cm}$ in width. And in order to have a comparison, a solar cell device without sintering in the furnace was also characterized. The results are shown in the Table 8.2. The measured I-V curve and the corresponding results is shown in Fig. 8.8.

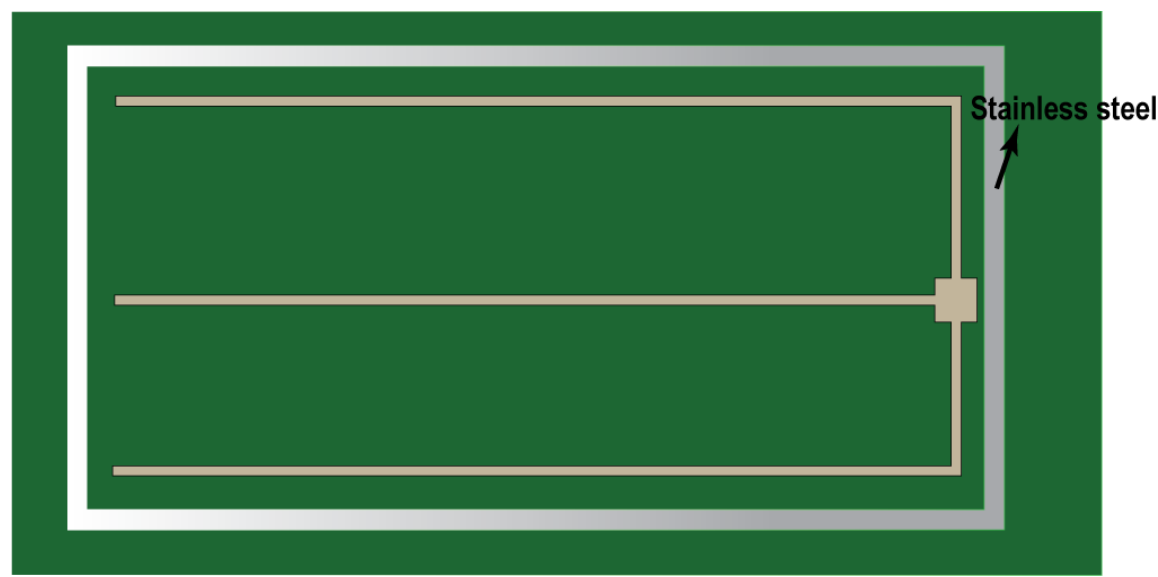

Figure 8.7: Schematic of the isolated solar cell. 
From the measured results, it can be seen that the solar cell without sintering gives a very bad result with very low short-circuit current, which indicates a very high series resistance. The sintered solar samples have good performance with an efficiency around $11-12 \%$. This results has proved the functionization of the LIFT line by furnace curing process.

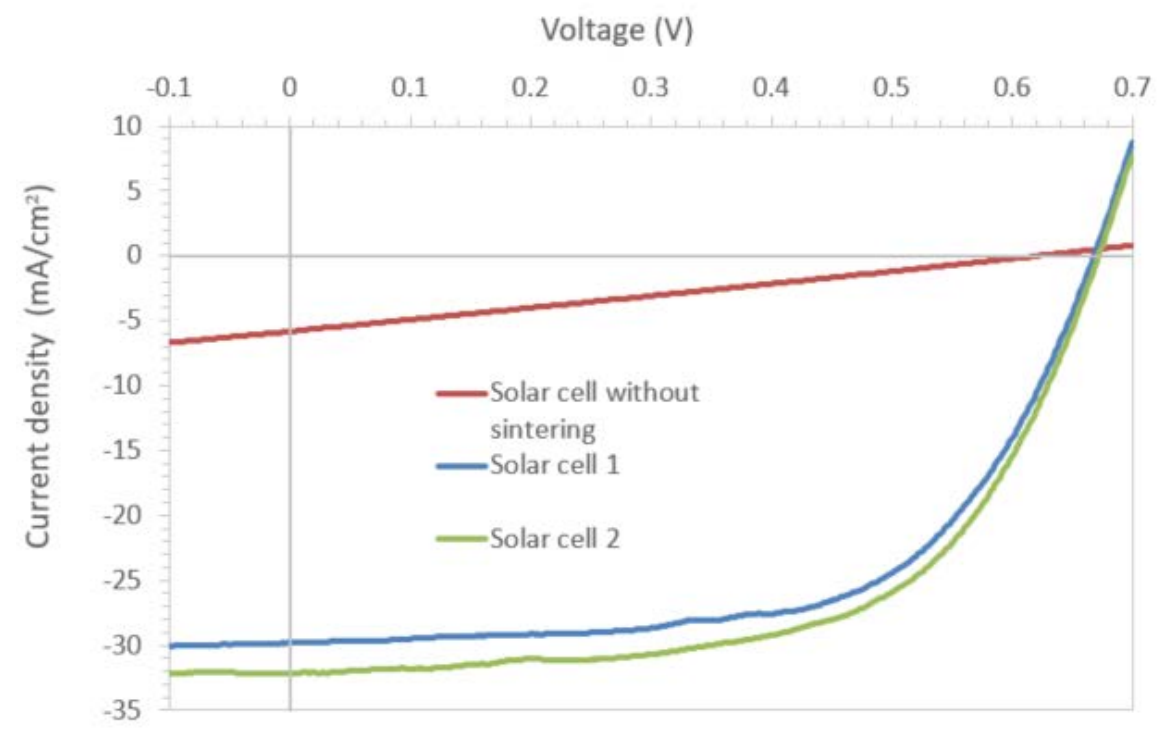

Figure 8.8: I-V curve of the CIGS solar cell sintered in the furnace.

\begin{tabular}{|c|c|c|c|c|c|c|c|c|}
\hline $\begin{array}{c}\text { Solar } \\
\text { cell } \\
\text { sample }\end{array}$ & $I_{s c}(A)$ & $\begin{array}{c}J_{s c} \\
\left(\mathrm{~mA} / \mathrm{cm}^{2}\right)\end{array}$ & $V_{o c}(\mathrm{~V})$ & $I_{m}(A)$ & $V_{m}(V)$ & $\begin{array}{c}P_{\max } \\
(W)\end{array}$ & $\begin{array}{c}F F \\
(\%)\end{array}$ & $\begin{array}{c}\text { Efficiency } \\
(\%)\end{array}$ \\
\hline 1 & 0.063 & 29.8 & 0.67 & 0.052 & 0.0494 & 0.0257 & 61.6 & 11.11 \\
\hline 2 & 0.067 & 32.1 & 0.67 & 0.054 & 0.0498 & 0.0272 & 60.2 & 11.78 \\
\hline
\end{tabular}

Table 8.2: CIGS solar cell characterization results with solar simulator.

The second test is to cure the solar cell (sample 3) contacts by CW laser. The CW laser employed in this process was the Millenia green laser with a spot size around $35 \mu \mathrm{m}$. Since the spot size of the laser is smaller than the line width (about $150 \mu \mathrm{m}$ ). The LIFT line was cured step by step with a low power and low scan speed in order to avoid the misalignment of the laser scan path and the damage to the CIGS solar cell substrate. The first step is to cure only the square pad, and then all the three finger lines were cured one by one. The next step is to cure the cure the two-connection line between the finger line 
on two sides and the square pad. After the first curing of the full contact area. This contact area was fully cured for another 3 times. And after curing the full cell for the fourth time, the sample was put in the furnace for a further treatment at $200^{\circ} \mathrm{C}$ for 1 hour. The curing process parameters and geometry of the LIFT lines are listed in Table 8.3.

The solar cell characterization was performed after each step of curing process. The measured I-V curve of after each laser curing process and the furnace treatment results is shown in Fig. 8.9. After the last step of furnace curing treatment, the ultimate solar cell characterization results are listed in Table 8.4.

\begin{tabular}{cc}
\hline Curing Parameters & Values \\
\hline \hline Laser power & $0.5 \mathrm{~W}$ \\
\hline Scan speed & $5 \mathrm{~mm} / \mathrm{s}$ \\
\hline Focus position & On Focus \\
\hline Spot size & $35 \mu \mathrm{m}$ \\
\hline Acceptor substrate & CIGS solar cell with TCO on the top layer \\
\hline Geometry of LIFT lines & \\
\hline width & $150-160 \mu \mathrm{m}$ \\
\hline height & $23-26 \mu \mathrm{m}$ \\
\hline
\end{tabular}

Table 8.3: Process parameters and geometry of the front contacts printed by LIFT on CIGS solar cell.

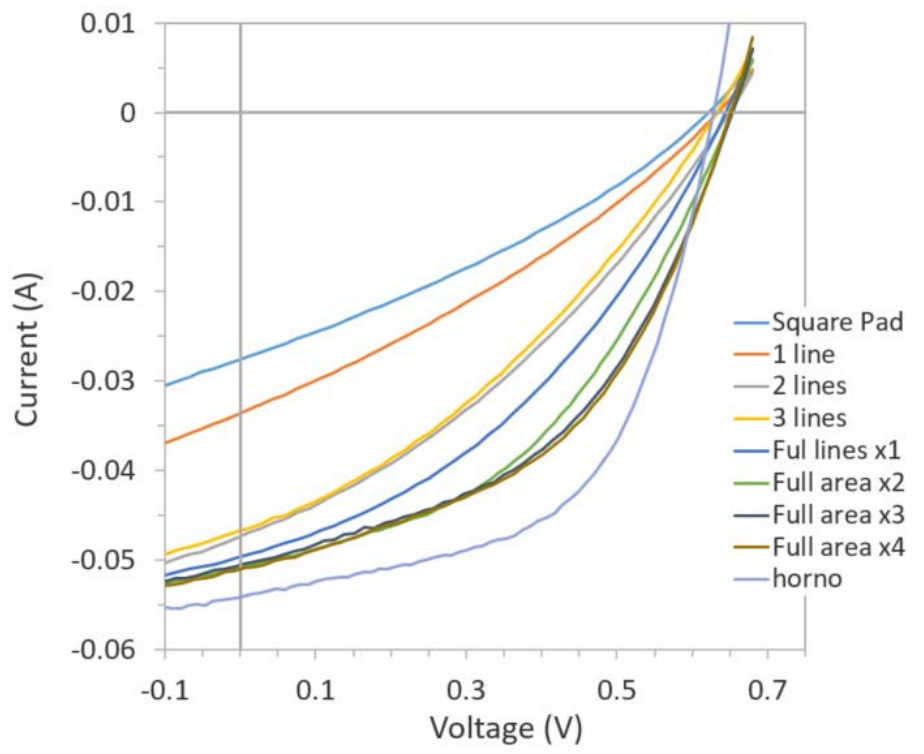

Figure 8.9: I-V curve of the CIGS solar cell sintered with CW laser step by step and finally in the furnace 


\begin{tabular}{|c|c|c|c|c|c|c|c|c|}
\hline $\begin{array}{c}\text { Solar } \\
\text { cell } \\
\text { sample }\end{array}$ & $I_{s c}(A)$ & $\begin{array}{c}J_{s c} \\
\left(\mathrm{~mA} / \mathrm{cm}^{2}\right)\end{array}$ & $\mathrm{V}_{\mathrm{oc}}(\mathrm{V})$ & $\mathrm{I}_{\mathrm{m}}(\mathrm{A})$ & $\mathrm{V}_{\mathrm{m}}(\mathrm{V})$ & $\begin{array}{c}\mathrm{P}_{\max } \\
(\mathrm{W})\end{array}$ & $\begin{array}{c}\mathrm{FF} \\
(\%)\end{array}$ & $\begin{array}{c}\text { Efficiency } \\
(\%)\end{array}$ \\
\hline 3 & 0.055 & 25.1 & 0.63 & 0.052 & 0.0494 & 0.0257 & 55 & 7.86 \\
\hline
\end{tabular}

Table 8.4: CIGS solar cell characterization results with solar simulator.

From Fig. 8.9 it can be seen that after each laser curing step, the short-circuit current value increases, which indicates the series resistance of the solar cell reduces. The shortcircuit current value increases a lot after the curing treatment of the third contact line which indicates the activation of the all finger lines for collecting current. However, after the fourth full area curing step, the IV curve doesn't change much. Since the furnace curing process provides a full contact area thermal treatment rather than the local surface curing by the laser, after the furnace curing treatment, the IV curve shifts again with increasing the short-circuit current and decreasing the open circuit voltage, which indicates that the series resistance of the solar cell reduces. It also indicates that there are more contact areas which is not been cured by laser and this laser curing process can be improved if a more precise vision system for positioning the laser beam on the surface of the contact is employed. Although the fill factor and cell efficiency of the third cell sample is lower than the other two sample ( 1 and 2 ), which can be caused by the cell itself, the contact structure on the cell or the uncured parts of the cell, the solar cell device has been functionalized. The concept of front contact metallization using the two-step laser processing (LIFT and selective laser curing) applied on the solar cell device has been proved. 


\section{Conclusion}

In this thesis, a variety of issues involving laser-induced forward transfer of high viscosity silver paste have been investigated from voxel printing to line printing using morphologically and experimental techniques. The new concept of front contact metallization combining laser-induced forward transfer (LIFT) and laser curing/sintering has been proved.

The influence of various process parameters such as laser fluence or pulse energy, gap distance, film thickness, and rheological properties of the silver paste to the voxel deposited on the acceptor were studied in chapter 5 . The paste contains micron-sized particles, and relatively thick donors were used in order to print voxels with high aspect ratios, leading to transfer mechanisms differing from those previously seen using silver nanopastes. These transfer mechanisms are studied through the morphology of transferred paste dots obtained with confocal microscopy and by time-resolved imaging of the transfer process.

Four transfer regimes are observed and defined: non-dot transfer, when the laser fluence is below the thickness-dependent transfer threshold; cluster-dot transfer, in which the transferred paste fragments into a small number of large clusters and the dot size increases with laser fluence; concrete-dot transfer, where the paste is thick enough and the gap is small enough to allow the protruding jet to touch the acceptor substrate before fragmentation; and explosive transfer, where high laser pulse energies cause a bursting transfer of paste that splashes on the acceptor. Concrete-dot transfer is the most useful for printing high-aspect-ratio dots, and the highest aspect ratios were achieved with pulse energies just above the threshold for transfer. By this coated method the coated silver paste thickness uncertainty is $10 \mu \mathrm{m}$. However, in the LIFT process, the film thickness tolerance should be within $5 \mu \mathrm{m}$. It is the first time to reveal the concrete-dot transfer mechanism of "bridge connection" in forming the voxel of silver paste.

In the ns-LIFT line printing section, it has been proved that with adequate parameterization process concerning the identification of laser pulse energy, spot size, 
overlapping of pulse, paste thickness and gap distance between donor and acceptor, long printed lines with high aspect radio (above 0.5 ) has been obtained using standard ns or ps laser sources integrated in state-of-the-art laser machines. It is also demonstrated that LIFT can be used to print freeform front grids in large areas without the need of any screen, which could be a fundamental tool to solve a key problem in BIPV: the customization of standard PV devices to fulfil the cost and aesthetic criteria imposed by the architectural design.

It has been also found that the wetting of the silver paste on the acceptor substrate is influenced by the roughness of the acceptor substrate surface. In order to increase the adhesion force, the liquid should highly wet the surface of the substrate. Increasing the surface roughness by texturing is an efficient method for solving this problem. When using a highly textured surface, the increased contact area provides a high adhesion force that prevents the paste from spreading along the surface, which shapes the voxel with a smaller width and larger height, i.e. an enhanced aspect ratio.

The transfer mechanism and regimes of ps-LIFT are observed to be the same as using ns-pulsed lasers in chapter 6. The voxel printing by ps-pulsed LIFT with different gap distance also reveals that the large gap distance leads to cluster when using laser pulse energies just above the transfer threshold value and the explosive transfer occurs when using higher energies. Concrete-dot transfer cannot be formed with large gap distance since the transferred silver paste cluster disaggregates into fragments before touch the acceptor surface.

In the line printing section, it is found that the pitch distance for overlapping the voxels in line scanning condition exists a threshold value above which the transfer will be ceased due to the escaped pressure through the laser-induced tunnel. Moreover, the wavelength of laser has weak influence on the silver paste line printing by LIFT. In addition, it has been the first time to demonstrate the feasibility of silver paste line printing with very high-speed (60 m/s or even higher) by using polygon scanner, which is favorable for the industrial fabrication. 
In chapter 7, it has been proved that laser curing/sintering of silver paste lines printed using LIFT can be achieved at different laser powers and scanning speeds, but there is a small parametric window for obtaining the desired properties (increased electrical conductivity and good mechanical adhesion), making it a very sensitive process.

It is demonstrated that fast scanning speeds with low laser power is need as a preheating process which can be also regarded as a curing process, to reduce the damage to the silver line and to have the best adhesion results then sintering. Lines need to be irradiated with short heating time but more scanning paths to neck all the silver particles in the solid state to obtain the best sintering performance. In that case, the electrical resistivity has been decreased by two orders of magnitude with a good morphology after laser sintering.

The geometry of the line is not affected by the laser curing and sintering when the lines are remained on the substrate, which preserves the aspect ratio obtained when printing lines using LIFT. It is proved that the selective laser curing and sintering can be combined with other laser direct writing printing techniques and be implemented in future photovoltaic industrial applications for developing an all-laser based metallization process.

A new method of metallization with two-step laser processing, i.e. laser-induced forward transfer (LIFT) and laser curing/sintering, has been proved on a final solar cell device in chapter 8 . Moreover, it is demonstrated that there is more space for the enhancement of the solar cell efficiency if the vision system for precisely positioning the laser scan track is improved. 


\section{Lines of Future Development}

After all the works developed in this thesis, several important lines of future development would be realized. One of the important line is to improve some LIFT set-ups in the processing system. In the donor preparation procedure, since the roughness of silver paste in the whole area of the donor substrate should be controlled precisely, the coating precision can be further improved by film applicator. A motorized Gap set-up can be substituted for sticking Kapton tapes manually in order to lifting the donor substrate with equilibrium force in two side, which can improve the homogeneity in the LIFT voxels or lines. The time-resolved imaging system can be added to LIFT system for the further transfer mechanism study. Moreover, a precision vision system can be added in the laser curing/sintering step for improving the positioning quality of the laser beam on the surface of the LIFT lines.

Secondly, the application of the knowledge acquired in the front grid metallization by the two-step laser processing combining LIFT and laser curing/sintering techniques applied on the CIGS thin film layers can be also applied to the crystalline silicon photovoltaic technologies.

Another alternative for future is to capitalize the results obtained in LIFT analysis and the degree of knowledge acquired during the same, opening the door to their application in the bio-LIFT application, whose study has a significant growth in the recent few years

Lastly, the degree of customization achieved in the LIFT process allows to propose novel applications of the photovoltaic devices in the field of BIPV, especially of those which fulfill the aesthetic criteria imposed by the architectural design.

It is important to highlight that the research group to which the author belongs has already initiated works on all these areas. 


\section{List of Publications}

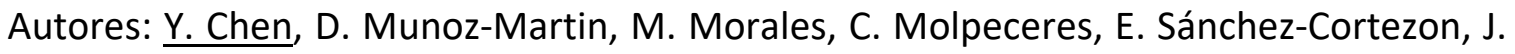
Murillo-Gutierrez

Título: Laser Induced Forward Transfer of High Viscosity Silver Paste for New Metallization Methods in Photovoltaic and Flexible Electronics Industry

Revista: Physics Procedia, 83 (2016) 204-210.

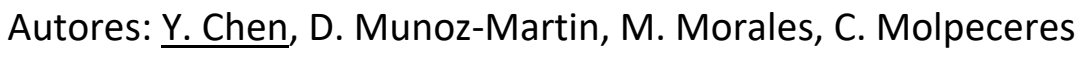

Título: Influence of the acceptor roughness on the aspect ratio of silver paste lines printed by Laser-induced forward transfer

Revista: Results in Physics, in preparation.

Autores: D. Munoz-Martin, C.F. Brasz, Y. Chen, M. Morales, C.B. Arnold, C. Molpeceres Título: Laser-induced forward transfer of high-viscosity silver pastes

Revista: Applied Surface Science, 366 (2016) 389-396.

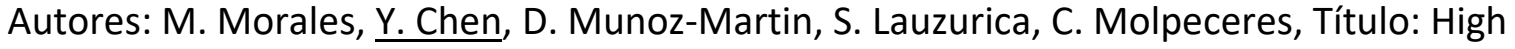
volume transfer of high viscosity silver pastes using laser direct-write processing for screen printing of c-Si cells

Revista: Proc. SPIE 9351 (2015) 93510B

\section{Conferences}

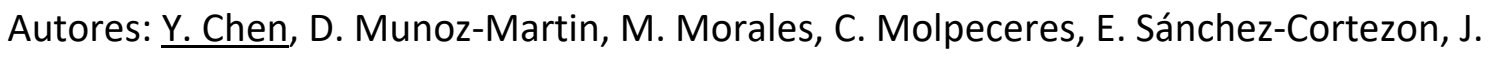
Murillo-Gutierrez

Congreso: 9th International Conference on Photonic Technologies LANE 2016

Ciudad: Fürtz (Alemania) Fechas: 19-22 Sep (2016)

Comunicación: Oral

Título: Laser induced forward transfer of high viscosity silver paste for new metallization methods in photovoltaic and flexible electronics industry

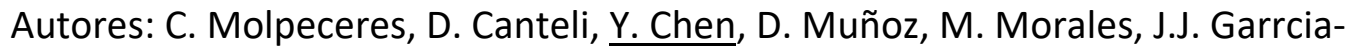

Ballesteros, S. Lauzurica

Congreso: CLEO: Applications and Technology 2016

Ciudad: San Jose, California (EEUU) Fechas: 5-10 June 2016

Título: Laser based Processes for Product Customization in Building Integrated Photovoltaics 
Comunicación: Oral

Publicación: Proceedings Conference on Lasers and Electro-Optics (2016) Paper:

ATU1K.5. ISBN: 978-1-943580-11-8

Autores: D. Munoz-Martin, C. F. Brasz, Y. Chen, M. Morales, C. B. Arnold, and C.Molpeceres

Congreso: Photonics West 2016

Lugar: San Francisco (EE.UU.) Fechas: 13-18 Feb (2016)

Comunicación: Oral

Título: Characterization of transfer regimes of high-viscosity silver pastes printed by LIFT

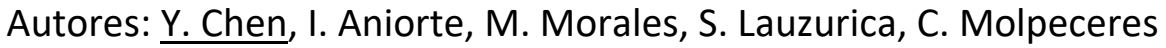

Congreso: 29st European Photovoltaic Solar Energy Conference and Exhibition EUPVSEC 2015

Lugar: Amsterdam (Holanda) Fecha: 22-24 septiembre 2014

Comunicación: Poster

Título: Laser Induced Forward Transfer of Silver Pastes for Printing of Fingers in c-Si Cells Publicado: Proceedings of the international Conference held in Amsterdam, Holand Paper 2CV.4

Autores: D. Munoz-Martin, Y. Chen, M. Morales, C. Molpeceres, M. Grishin Congreso: 31st European Photovoltaic Solar Energy Conference and Exhibition EUPVSEC 2015

Lugar: Hamburgo (Alemania) Fecha: 14-18 septiembre 2015

Comunicación: Poster

Título: Metallization of silicon solar cells using ps- and ns-pulsed lasers

Publicado: Proceedings of the international Conference held in Hamburg, Germany

Paper 2CV.4.20

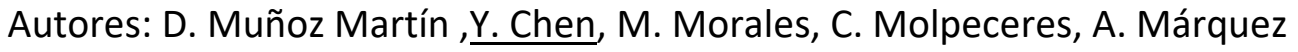

Título: Laser printing and curing/sintering of silver paste lines for solar cell metallization Congreso: Lasers in Manufacturing Conference 2015.

Lugar: Munich (Alemania). Fecha: 22-25 junio 2015

Publicado en Actas: Lasers in Manufacturing Proceedings 2015 http://www.wlt.de/lim/. 


\section{Reference}

[1] J. Bartsch, A. Mondon, B. Godejohann, M. Hörteis, S. Glunz, Advanced front side metallization for crystalline silicon solar cells based on a fully plated contact, in.

[2] H. Ishihara, W. Chen, Y.-C. Chen, S. Sarang, N. De Marco, O. Lin, S. Ghosh, V. Tung, Electrohydrodynamically Assisted Deposition of Efficient Perovskite Photovoltaics, Advanced Materials Interfaces, (2016) n/a-n/a.

[3] D.-Y. Shin, S.-S. Yoo, H.-e. Song, H. Tak, D. Byun, Electrostatic-Force-Assisted Dispensing Printing to Construct High-Aspect-Ratio of 0.79 Electrodes on a Textured Surface with Improved Adhesion and Contact Resistivity, Scientific Reports, 5 (2015) 16704.

[4] X. Baomin, K. Littau, J. Zesch, D. Fork, Front side metallization of crystalline silicon solar cells using selectively laser drilled contact openings, in: Photovoltaic Specialists Conference (PVSC), 2009 34th IEEE, 2009, pp. 000517-000522.

[5] A. Mette, P.L. Richter, M. Hörteis, S.W. Glunz, Metal aerosol jet printing for solar cell metallization, Progress in Photovoltaics: Research and Applications, 15 (2007) 621-627.

[6] A. Ebong, C. Nian, Metallization of crystalline silicon solar cells: A review, in: High Capacity Optical Networks and Enabling Technologies (HONET), 2012 9th International Conference on, 2012, pp. 102-109.

[7] C.B. Arnold, P. Serra, A. Piqué, Laser Direct-Write Techniques for Printing of Complex Materials, MRS Bulletin, 32 (2007) 23-31.

[8] T.L. M. Nagel, Laser-induced forward transfer for the fabrication of devices, in: Nanomaterials: Processing and Characterization with Lasers, Wiley-VCH, 2012, pp. 255-316. [9] G. Hennig, T. Baldermann, C. Nussbaum, M. Rossier, A. Brockelt, L. Schuler, G. Hochstein, Lasersonic ${ }^{\circledR}$ LIFT Process for Large Area Digital Printing, Journal of Laser Micro/Nanoengineering, 7(3) (2012) 289-305.

[10] T.C. Roder, E. Hoffmann, J.R. Kohler, J.J.H. Werner, $30 \mu \mathrm{m}$ wide contacts on silicon cells by laser transfer, in: Photovoltaic Specialists Conference (PVSC), 2010 35th IEEE, IEEE, 2010, pp. 003597-003599.

[11] S.A. Mathews, N.A. Charipar, R.C. Auyeung, H. Kim, A. Piqué, Laser forward transfer of solder paste for microelectronics fabrication, in, 2015, pp. 93510Y-93510Y-93519.

[12] E. Breckenfeld, H. Kim, R.C.Y. Auyeung, N. Charipar, P. Serra, A. Piqué, Laser-induced forward transfer of silver nanopaste for microwave interconnects, Applied Surface Science, 331 (2015) 254-261.

[13] M. Morales, Y. Chen, D. Munoz-Martin, S. Lauzurica, C. Molpeceres, High volume transfer of high viscosity silver pastes using laser direct-write processing for screen printing of c-Si cells, in: Proc. SPIE 9351, 2015, pp. 93510B-93510B-93517.

[14] H. Kim, G.P. Kushto, C.B. Arnold, Z.H. Kafafi, A. Piqué, Laser processing of nanocrystalline TiO2 films for dye-sensitized solar cells, Applied Physics Letters, 85 (2004) 464-466.

[15] M. Feinaeugle, A.P. Alloncle, P. Delaporte, C.L. Sones, R.W. Eason, Time-resolved shadowgraph imaging of femtosecond laser-induced forward transfer of solid materials, Applied Surface Science, 258 (2012) 8475-8483.

[16] M. Duocastella, J.M. Fernández-Pradas, P. Serra, J.L. Morenza, Jet formation in the laser forward transfer of liquids, Appl. Phys. A, 93 (2008) 453-456.

[17] M. Colina, M. Duocastella, J.M. Fernández-Pradas, P. Serra, J.L. Morenza, Laser-induced forward transfer of liquids: Study of the droplet ejection process, Journal of Applied Physics, 99 (2006) 084909. 
[18] D.-Y. Shin, J.-Y. Seo, H. Tak, D. Byun, Bimodally dispersed silver paste for the metallization of a crystalline silicon solar cell using electrohydrodynamic jet printing, Solar Energy Materials and Solar Cells, 136 (2015) 148-156.

[19] D. Munoz-Martin, C.F. Brasz, Y. Chen, M. Morales, C.B. Arnold, C. Molpeceres, Laserinduced forward transfer of high-viscosity silver pastes, Applied Surface Science, 366 (2016) 389396.

[20] J.L. Gray, The Physics of the Solar Cell, in: Handbook of Photovoltaic Science and Engineering, John Wiley \& Sons, Ltd, 2011, pp. 82-129.

[21] M.A. Green, Solar cell fill factors: General graph and empirical expressions, Solid-State Electronics, 24 (1981) 788-789.

[22] D. Erath, A. Filipović, M. Retzlaff, A.K. Goetz, F. Clement, D. Biro, R. Preu, Advanced screen printing technique for high definition front side metallization of crystalline silicon solar cells, Solar Energy Materials and Solar Cells, 94 (2010) 57-61.

[23] M. Galiazzo, A. Voltan, E. Bortoletto, M. Zamuner, M. Martire, O. Borsato, M. Bertazzo, D. Tonini, Fine Line Double Printing and Advanced Process Control for Cell Manufacturing, Energy Procedia, 67 (2015) 116-125.

[24] D. Buzby ${ }^{1}$, A. Dobie, Fine line screen printing of thick film pastes on silicon solar cells.

[25] B.R. Olaisen, A. Holt, E.S. Marstein, E. Sauar, A. Shaikh, K. McVicker, J. Salami, H. Miranda, S.S. Kim, Hot-melt screen-printing of front contacts on crystalline silicon solar cells, in:

Photovoltaic Specialists Conference, 2005. Conference Record of the Thirty-first IEEE, 2005, pp. 1084-1087.

[26] J. Zhao, A. Wang, P.P. Altermatt, S.R. Wenham, M.A. Green, 24\% efficient perl silicon solar cell: Recent improvements in high efficiency silicon cell research, Solar Energy Materials and Solar Cells, 41-42 (1996) 87-99.

[27] P.S. Aakella, S. Saravanan, S.S. Joshi, C.S. Solanki, Pre-metallization processes for c-Si solar cells, Solar Energy, 97 (2013) 388-397.

[28] J. Zhao, A. Wang, M.A. Green, 24.5\% Efficiency silicon PERT cells on MCZ substrates and $24.7 \%$ efficiency PERL cells on FZ substrates, Progress in Photovoltaics: Research and Applications, 7 (1999) 471-474.

[29] M.A. Green, C.M. Chong, F. Zhang, A. Sproul, J. Zolper, S.R. Wenham, 20\% efficient laser grooved, buried contact silicon solar cells, in: Photovoltaic Specialists Conference, 1988., Conference Record of the Twentieth IEEE, 1988, pp. 411-414 vol.411.

[30] J.A. Jensen, P. Møller, T. Bruton, N. Mason, R. Russell, J. Hadley, P. Verhoeven, A. Matthewson, Electrochemical deposition of buried contacts in high-efficiency crystalline silicon photovoltaic cells, Journal of the Electrochemical Society, 150 (2003) G49-G57.

[31] G. Yao, J. Zhao, J. Cotter, Metal stencil-printed selective emitter silicon solar cell fabrication on CZ wafer, in: Proceedings of the 20th European Photovoltaic Solar Energy Conference, 2005, pp. 749-752.

[32] A. Mette, New Concepts for Front Side Metallization of Industrial Silicon Solar Cells, Verlag Dr. Hut, 2007.

[33] P. Hahne, I.E. Reis, E. Hirth, D.M. Huljic, R. Preu, Pad printing - a novel thick-film technique of fine-line printing for solar cells, in: 2nd World Conference on Photovoltaic Solar Energy Conversion 1998; Proceedings of the international conference, Luxembourg: Office for Official Publications of the European Communitie, 1998. 
[34] M.Y. Ghannam., J. Poortmans., J.F. Nijs., Quantitative comparison between the performance of buried contact and of screenprinted contact industrial silicon solar cells, Kuwait Journal of Science and Engineering, 25 (1998) 363-377.

[35] K. Murata, Super-fine ink-jet printing for nanotechnology, in: MEMS, NANO and Smart Systems, 2003. Proceedings. International Conference on, 2003, pp. 346-349.

[36] K.F. Teng, R.W. Vest, Application of ink jet technology on photovoltaic metallization, IEEE Electron Device Letters, 9 (1988) 591-593.

[37] Y.T. Gizachew, L. Escoubas, J.J. Simon, M. Pasquinelli, J. Loiret, P.Y. Leguen, J.C. Jimeno, J. Martin, A. Apraiz, J.P. Aguerre, Towards ink-jet printed fine line front side metallization of crystalline silicon solar cells, Solar Energy Materials and Solar Cells, 95, Supplement 1 (2011) S70-S82.

[38] M.F.A.M.v. Hest, S.E. Habas, J.M. Underwood, R.M. Pasquarelli, P. Hersh, A. Miedaner, C.J. Curtis, D.S. Ginley, Direct write metallization for photovoltaic cells and scaling thereof, in: Photovoltaic Specialists Conference (PVSC), 2010 35th IEEE, 2010, pp. 003626-003628. [39] J.-U. Park, M. Hardy, S.J. Kang, K. Barton, K. Adair, D.k. Mukhopadhyay, C.Y. Lee, M.S. Strano, A.G. Alleyne, J.G. Georgiadis, P.M. Ferreira, J.A. Rogers, High-resolution electrohydrodynamic jet printing, Nat Mater, 6 (2007) 782-789. [40] T. Mattle, J. Shaw-Stewart, C.W. Schneider, T. Lippert, A. Wokaun, Laser induced forward transfer aluminum layers: Process investigation by time resolved imaging, Applied Surface Science, 258 (2012) 9352-9354.

[41] A. Piqué, D.B. Chriseya, J.M. Fitz-Geralda, R.A. McGilla, R.C.Y. Auyeunga, H.D. Wua, S. Lakeoua, V. Nguyena, R. Chunga, M. Duignana, Direct writing of electronic and sensor materials using a laser transfer technique, Journal of Materials Research, 15 (2000) 1872-1875.

[42] R. Modi, H.D. Wu, R.C.Y. Auyeung, C.M. Gilmore, D.B. Chrisey, Direct writing of polymer thick film resistors using a novel laser transfer technique, Journal of Materials Research, 16 (2011) 3214-3222.

[43] A. Piqué, D.B. Chrisey, R.C.Y. Auyeung, J. Fitz-Gerald, H.D. Wu, R.A. McGill, S. Lakeou, P.K. $\mathrm{Wu}, \mathrm{V}$. Nguyen, M. Duignan, A novel laser transfer process for direct writing of electronic and sensor materials, Appl. Phys. A, 69 (1999) S279-S284.

[44] D.B. Chrisey, A. Piqué, R.A. McGill, J.S. Horwitz, B.R. Ringeisen, D.M. Bubb, P.K. Wu, Laser Deposition of Polymer and Biomaterial Films, Chemical Reviews, 103 (2003) 553-576.

[45] H. Fukumura, Y. Kohji, K.-i. Nagasawa, H. Masuhara, Laser Implantation of Pyrene Molecules into Poly(methyl methacrylate) Films, Journal of the American Chemical Society, 116 (1994) 10304-10305.

[46] H. Fukumura, H. Uji-i, H. Banjo, H. Masuhara, D.M. Karnakis, N. Ichinose, S. Kawanishi, K. Uchida, M. Irie, Laser implantation of photochromic molecules into polymer films: a new approach towards molecular device fabrication, Applied Surface Science, 127-129 (1998) 761766.

[47] Z. Lin, L.V. Zhigilei, V. Celli, Electron-phonon coupling and electron heat capacity of metals under conditions of strong electron-phonon nonequilibrium, Physical Review B, 77 (2008) 075133.

[48] C.F. Brasz, C.B. Arnold, H.A. Stone, J.R. Lister, Early-time free-surface flow driven by a deforming boundary, Journal of Fluid Mechanics, 767 (2015) 811-841.

[49] Z. Kántor, Z. Tóth, T. Szörényi, Metal pattern deposition by laser-induced forward transfer, Applied Surface Science, 86 (1995) 196-201. 
[50] V. Schultze, M. Wagner, Laser-induced forward transfer of aluminium, Applied Surface Science, 52 (1991) 303-309.

[51] H. Yamada, T. Sano, T. Nakayama, I. Miyamoto, Optimization of laser-induced forward transfer process of metal thin films, Applied Surface Science, 197-198 (2002) 411-415.

[52] S. Lätsch, H. Hiraoka, W. Nieveen, J. Bargon, Interface study on laser-induced material transfer from polymer and quartz surfaces, Applied Surface Science, 81 (1994) 183-194.

[53] B. Tan, K. Venkatakrishnan, K.G. Tok, Selective surface texturing using femtosecond pulsed laser induced forward transfer, Applied Surface Science, 207 (2003) 365-371.

[54] H. Esrom, J.-Y. Zhang, U. Kogelschatz, A.J. Pedraza, New approach of a laser-induced forward transfer for deposition of patterned thin metal films, Applied Surface Science, 86 (1995) 202-207.

[55] C.W. Visser, R. Pohl, C. Sun, G.-W. Römer, B. Huis in 't Veld, D. Lohse, Toward 3D Printing of Pure Metals by Laser-Induced Forward Transfer, Advanced Materials, 27 (2015) 4087-4092.

[56] B. Thomas, A.P. Alloncle, P. Delaporte, M. Sentis, S. Sanaur, M. Barret, P. Collot, Experimental investigations of laser-induced forward transfer process of organic thin films, Applied Surface Science, 254 (2007) 1206-1210.

[57] D. Young, R.C.Y. Auyeung, A. Piqué, D.B. Chrisey, D.D. Dlott, Plume and jetting regimes in a laser based forward transfer process as observed by time-resolved optical microscopy, Applied Surface Science, 197-198 (2002) 181-187.

[58] J.A. Barron, H.D. Young, D.D. Dlott, M.M. Darfler, D.B. Krizman, B.R. Ringeisen, Printing of protein microarrays via a capillary-free fluid jetting mechanism, PROTEOMICS, 5 (2005) 41384144.

[59] C. Unger, M. Gruene, L. Koch, J. Koch, B.N. Chichkov, Time-resolved imaging of hydrogel printing via laser-induced forward transfer, Appl. Phys. A, 103 (2011) 271-277.

[60] M. Duocastella, A. Patrascioiu, V. Dinca, J.M. Fernández-Pradas, J.L. Morenza, P. Serra, Study of liquid deposition during laser printing of liquids, Applied Surface Science, 257 (2011) 5255-5258.

[61] J.M. Cheny, K. Walters, Rheological influences on the splashing experiment, Journal of NonNewtonian Fluid Mechanics, 86 (1999) 185-210.

[62] T. Mao, D.C.S. Kuhn, H. Tran, Spread and rebound of liquid droplets upon impact on flat surfaces, AIChE Journal, 43 (1997) 2169-2179.

[63] M. Rein, Phenomena of liquid drop impact on solid and liquid surfaces, Fluid Dynamics Research, 12 (1993) 61-93.

[64] I.V. Roisman, R. Rioboo, C. Tropea, Normal impact of a liquid drop on a dry surface: model for spreading and receding, Proceedings of the Royal Society of London. Series A: Mathematical, Physical and Engineering Sciences, 458 (2002) 1411-1430.

[65] D.B. van Dam, C. Le Clerc, Experimental study of the impact of an ink-jet printed droplet on a solid substrate, Physics of Fluids, 16 (2004) 3403-3414.

[66] M. Brown, N. Kattamis, C. Arnold, Time-resolved dynamics of laser-induced micro-jets from thin liquid films, Microfluid Nanofluid, 11 (2011) 199-207.

[67] M. Duocastella, H. Kim, P. Serra, A. Piqué, Optimization of laser printing of nanoparticle suspensions for microelectronic applications, Appl. Phys. A, 106 (2012) 471-478.

[68] S.A. Mathews, R.C.Y. Auyeung, H. Kim, N.A. Charipar, A. Piqué, High-speed video study of laser-induced forward transfer of silver nano-suspensions, Journal of Applied Physics, 114 (2013) 064910. 
[69] M. Colina, P. Serra, J.M. Fernández-Pradas, L. Sevilla, J.L. Morenza, DNA deposition through laser induced forward transfer, Biosensors and Bioelectronics, 20 (2005) 1638-1642.

[70] M. Baum, H. Kim, I. Alexeev, A. Piqué, M. Schmidt, Generation of transparent conductive electrodes by laser consolidation of LIFT printed ITO nanoparticle layers, Appl. Phys. A, 111 (2013) 799-805.

[71] J. Shaw-Stewart, T. Mattle, T. Lippert, M. Nagel, F. Nüesch, A. Wokaun, The optimisation of the laser-induced forward transfer process for fabrication of polyfluorene-based organic lightemitting diode pixels, Applied Surface Science, 278 (2013) 341-346.

[72] C. Constantinescu, A.K. Diallo, A. D'Aleo, F. Fages, C. Videlot-Ackermann, P. Delaporte, A.-P. Alloncle, Laser-induced forward transfer of a bis-pyrene compound for OTFTs, Applied Surface Science, 336 (2015) 133-137.

[73] L. Rapp, C. Constantinescu, Y. Larmande, A.K. Diallo, C. Videlot-Ackermann, P. Delaporte, A.P. Alloncle, Functional multilayered capacitor pixels printed by picosecond laser-induced forward transfer using a smart beam shaping technique, Sensors and Actuators A: Physical, 224 (2015) 111-118.

[74] M. Colina, A. Morales-Vilches, C. Voz, I. Martín, P. Ortega, A. Orpella, G. López, R. Alcubilla, Laser Induced Forward Transfer for front contact improvement in silicon heterojunction solar cells, Applied Surface Science, 336 (2015) 89-95.

[75] A. Piqué, H. Kim, R.C.Y. Auyeung, I. Beniam, E. Breckenfeld, Laser-induced forward transfer (LIFT) of congruent voxels, Applied Surface Science.

[76] M.I. Sanchez-Aniorte, B. Mouhamadou, A.P. Alloncle, T. Sarnet, P. Delaporte, Laser-induced forward transfer for improving fine-line metallization in photovoltaic applications, Appl. Phys. A, 122 (2016) 1-5.

[77] T.C. Röder, E. Hoffmann, B. Konrad, J.R. Köhler, Low Temperature Laser Metallization for Silicon Solar Cells, Energy Procedia, 8 (2011) 552-557.

[78] D.-H. Kim, S.-S. Ryu, D. Shin, J.-H. Shin, J.-J. Jeong, H.-J. Kim, H.S. Chang, The fabrication of front electrodes of Si solar cell by dispensing printing, Materials Science and Engineering: B, 177 (2012) 217-222.

[79] M. Neidert, W. Zhang, D. Zhang, A. Kipka, Screen-printing simulation study on solar cell front side AG paste, in: Photovoltaic Specialists Conference, 2008. PVSC '08. 33rd IEEE, 2008, pp. 1-4.

[80] M. Makrygianni, I. Kalpyris, C. Boutopoulos, I. Zergioti, Laser induced forward transfer of Ag nanoparticles ink deposition and characterization, Applied Surface Science, 297 (2014) 40-44. [81] M. Duocastella, J.M. Fernández-Pradas, J.L.M.P. Serra, Sessile droplet formation in the laser-induced forward transfer of liquids: A time-resolved imaging study, Thin Solid Films, 518 (2010) 5321-5325.

[82] M. Duocastella, J.M. Fernández-Pradas, J. Domínguez, P. Serra, J.L. Morenza, Printing biological solutions through laser-induced forward transfer, Appl. Phys. A, 93 (2008) 941-945. [83] M.S. Brown, C.F. Brasz, Y. Ventikos, C.B. Arnold, Impulsively actuated jets from thin liquid films for high-resolution printing applications, Journal of Fluid Mechanics, 709 (2012) 341-370. [84] J. Wang, R.C.Y. Auyeung, H. Kim, N.A. Charipar, A. Piqué, Three-Dimensional Printing of Interconnects by Laser Direct-Write of Silver Nanopastes, Advanced Materials, 22 (2010) 44624466.

[85] Y. Chen., M.I.S. Aniorte., M. Morales., S. Lauzurica., C. Molpeceres., Laser Induced Forward Transfer of Silver Pastes for Printing of Fingers in c-Si Cells, in: 29th European Photovoltaic Solar Energy Conference and Exhibition, Amsterdam (Holanda), 2014, pp. 1406 - 1409. 
[86] D. Munoz-Martin., Y. Chen., M. Morales., C. Molpeceres., M. Grishin., Metallization of Silicon Solar Cells Using ps- and ns-Pulsed Lasers, in: 31st European Photovoltaic Solar Energy Conference and Exhibition, 2015, pp. 962 - 964.

[87] J.M. Liu, Simple technique for measurements of pulsed Gaussian-beam spot sizes, Opt. Lett., 7 (1982) 196-198.

[88] Q. Che, H. Yang, L. Lu, Y. Wang, A new environmental friendly silver front contact paste for crystalline silicon solar cells, Journal of Alloys and Compounds, 549 (2013) 221-225.

[89] Z. Dong, J. Moyer, Z. Weiming, Front contact pastes with increased aspect ratio to achieve higher efficiency on screen printed solar cells, in: Photovoltaic Specialists Conference (PVSC), 2009 34th IEEE, 2009, pp. 001321-001324.

[90] C.-H. Lin, S.-P. Hsu, W.-C. Hsu, Silicon solar cells: structural properties of Ag-Contacts/SiSubstrate.

[91] J. Qin, W. Zhang, S. Bai, Z. Liu, Study on the sintering and contact formation process of silver front side metallization pastes for crystalline silicon solar cells, Applied Surface Science, 376 (2016) 52-61.

[92] J. Zhou, N. Xu, H. Yang, Q. Zhang, Effect of Ag Powder and Glass Frit in Ag Paste on Front Contract of Silicon Solar Cells, Procedia Engineering, 94 (2014) 1-5.

[93] DuPont, Photovoltaic metallization pastes, in, <http://www.dupont.com/products-andservices/solar-photovoltaic-materials/photovoltaic-metallization-pastes.html>.

[94] A. Rosidah, M.S. Sabrina, Rheological Behaviors and Their Correlation with Printing

Performance of Silver Paste for LTCC Tape, in: D.J.D. Vicente (Ed.) Rheology, 2012.

[95] H.-W. Lin, C.-P. Chang, W.-H. Hwu, M.-D. Ger, The rheological behaviors of screen-printing pastes, Journal of Materials Processing Technology, 197 (2008) 284-291.

[96] H.A. Barnes, Thixotropy-a review, Journal of Non-Newtonian Fluid Mechanics, 70 (1997) 133.

[97] M.S. Brown, N.T. Kattamis, C.B. Arnold, Time-resolved study of polyimide absorption layers for blister-actuated laser-induced forward transfer, Journal of Applied Physics, 107 (2010) 083103.

[98] D.K. Schroder, Contact Resistance and Schottky Barriers, in: Semiconductor Material and Device Characterization, John Wiley \& Sons, Inc., 2005, pp. 127-184.

[99] P. Serra, M. Duocastella, J.M. Fernández-Pradas, J.L. Morenza, Liquids microprinting through laser-induced forward transfer, Applied Surface Science, 255 (2009) 5342-5345.

[100] V. Dinca, M. Farsari, D. Kafetzopoulos, A. Popescu, M. Dinescu, C. Fotakis, Patterning parameters for biomolecules microarrays constructed with nanosecond and femtosecond UV lasers, Thin Solid Films, 516 (2008) 6504-6511.

[101] M. Duocastella, J.M. Fernández-Pradas, J.L. Morenza, P. Serra, Time-resolved imaging of the laser forward transfer of liquids, Journal of Applied Physics, 106 (2009) 084907.

[102] J. Yan, Y. Huang, C. Xu, D.B. Chrisey, Effects of fluid properties and laser fluence on jet formation during laser direct writing of glycerol solution, Journal of Applied Physics, 112 (2012) 083105.

[103] N.T. Kattamis, M.S. Brown, C.B. Arnold, Finite element analysis of blister formation in laser-induced forward transfer, Journal of Materials Research, 26 (2011) 2438-2449.

[104] C.F. Brasz, Liquid jet formation in laser-induced forward transfer, in: Mechanical and aerospace engineering, Princeton university, 2015, pp. 206. 
[105] J.J. García-Ballesteros, S. Lauzurica, M. Morales, T. del Caño, D. Valencia, L. Casado, J.L. Balenzategui, C. Molpeceres, Silicon PV module customization using laser technology for new BIPV applications, in, 2014, pp. 91800H-91800H-91811.

[106] C. Florian, F. Caballero-Lucas, J.M. Fernández-Pradas, R. Artigas, S. Ogier, D. Karnakis, P. Serra, Conductive silver ink printing through the laser-induced forward transfer technique, Applied Surface Science, 336 (2015) 304-308.

[107] C. Florian, F. Caballero-Lucas, J.M. Fernández-Pradas, S. Ogier, L. Winchester, D. Karnakis, R. Geremia, R. Artigas, P. Serra, Printing of silver conductive lines through laser-induced forward transfer, Applied Surface Science, 374 (2016) 265-270.

[108] I. Tobías, C. del Cañizo, J. Alonso, Crystalline Silicon Solar Cells and Modules, in: Handbook of Photovoltaic Science and Engineering, John Wiley \& Sons, Ltd, 2011, pp. 265-313.

[109] Y. Lin, E. Van Kerschaver, K. Cabanas-Holmen, Laser sintering of screen-printed silver paste for silicon solar cells, in: 2013 IEEE 39th Photovoltaic Specialists Conference (PVSC), IEEE, 2013, pp. 3445-3447.

[110] B.J. Perelaer, A.W.M. de Laat, C.E. Hendriks, U.S. Schubert, Inkjet-printed silver tracks: low temperature curing and thermal stability investigation, Journal of Materials Chemistry, 18 (2008) 3209-3215.

[111] A.J. Lopes, I.H. Lee, E. MacDonald, R. Quintana, R. Wicker, Laser curing of silver-based conductive inks for in situ 3D structural electronics fabrication in stereolithography, Journal of Materials Processing Technology, 214 (2014) 1935-1945.

[112] K. Tae-Yeop, H. Jun-Young, M. Seung-Jae, Laser Curing of the Silver/Copper Nanoparticle Ink via Optical Property Measurement and Calculation, Japanese Journal of Applied Physics, 49 (2010) 05EA09.

[113] K. Maekawa, K. Yamasaki, T. Niizeki, M. Mita, Y. Matsuba, N. Terada, H. Saito, Laser sintering of silver nanoparticles for electronic use, in: Materials Science Forum, Trans Tech Publ, 2010, pp. 2085-2090.

[114] Z. Zhang, Processing and Characterization of Micro-scale and Nanscale Silver Paste for Power Semiconductor Device Attachment, in, Virginia Polytechnic Institute and State University, 2005.

[115] G. Römer, J. Meijer, Metal surface temperature induced by moving laser beams, Optical and quantum electronics, 27 (1995) 1397-1406.

[116] P. Coussot, Yield stress fluid flows: A review of experimental data, Journal of NonNewtonian Fluid Mechanics, 211 (2014) 31-49.

[117] S.D. Holdsworth, Rheological models used for the prediction of the flow properties of food products: a literature review, Food and bioproducts processing : transactions of the Institution of Chemical Engineers, Part C., 71 (1993) 139-179.

[118] P. Coussot, Q.D. Nguyen, H.T. Huynh, D. Bonn, Viscosity bifurcation in thixotropic, yielding fluids, Journal of Rheology, 46 (2002) 573-589. 


\section{Annex}

\section{Brief introduction of fluids}

The Newtonian fluid, defined as a fluid in which the shear stress is linearly proportional to the local strain rate (shear rate), gives the simplest mathematical model of fluid. In the Newtonian fluid, the viscosity (specifically, the dynamic viscosity) is regarded as a constant, saying the resistance to gradual deformation by shear stress or tensile stress doesn't change. The shear stress $\tau$, which causes the friction loss in Newtonian fluid, depends on the viscosity $\mu$ of the fluid and the shear rate $\dot{\gamma}$ :

$$
\tau=\mu \cdot \dot{\gamma}=\mu \cdot \frac{d v}{d y}
$$

The non-Newtonian fluid like high viscous metal inks, hydrogel, silver nanopaste, metal paste and solder paste etc. present not only very different rheological behaviors compared to those low viscosity Newtonian fluids in the LIFT process but also have a different deposition and formation mechanism which affect the final morphology of the voxel or line. For the printing process in the thick-film metallization of solar cell, the paste like silver paste or solder paste must have a time-dependent shear thinning property, which is thixotropic.

For the metal paste, its viscosity reduces as a function of shear rate since the long chain molecules change the orientation in the paste vehicle. This rheological behavior can be described using Ostwals-de Waele's Power-Law for an approximation:

$$
\begin{gathered}
\mu(\dot{\gamma})=c \cdot \dot{\gamma}^{m-1} \\
\tau(\dot{\gamma})=c \cdot \dot{\gamma}^{m}
\end{gathered}
$$


where $\mu$ is the viscosity, the power-law index $\mathrm{m}<1$ indicates the shear thinning behaviors. These pastes are high viscous under static conditions and will become thin (less viscosity) when apply a shear stress over time, and finally reach an equilibrium value (plateau value). Some of them take a fixed time to return to a more viscous state and there is also some thixotropic fluid return to a gel state almost instantly, which is sometimes called nonNewtonian pseudoplastic fluid with time-dependent change in viscosity. The typical thixotropic behavior of shearing in short and long recovery time is illustrated in Fig. A.1 The silver paste and the solder paste are thixotropic fluids.

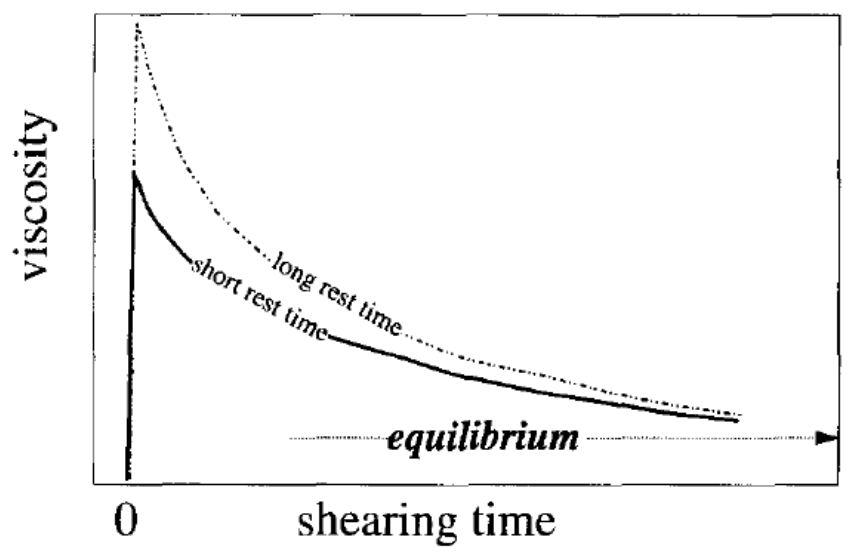

Figure A.1: Typical thixotropic behavior of shearing in short and long recovery time [96].

In particular, silver paste is a thixotropic yield stress fluid which features a fluid behavior (plastic or viscous deformation) only if they are above a critical stress value and when the stress is removed it will soon recover to its original "soft-solid" state without sacrificing its intrinsic mechanical properties [116]. For a weakly thixotropic yield stress fluid, the rheological behavior in steady state simple shear can be described by the Herschel-Bulkley model [117] as follows:

$$
\tau<\tau_{c} \Rightarrow \dot{\gamma}=0 \text { (solid regime) }
$$




$$
\tau<\tau_{c} \Rightarrow \tau=\tau_{c}+k \dot{\gamma}^{n} \text { (liquid regime) }
$$

where $\tau$ is the shear stress amplitude, $\dot{\gamma}$ is the shear rate amplitude, $\tau_{c}$ is the yield stress which associated with the transient from solid regime to liquid regime of the paste, and $k$ and $n$ values related with material property. The $n$ is normally lies in the range [0.3;0.5]. And the typical yield stress values lie between 1 and $100 \mathrm{~Pa}$. Particularly, when $n=1$, the model is changed to Bingham model followed by Newtonian behavior.

For fitting the experimental data, $\mathrm{S}$. Thibert et al. use a derived model as follows:

$$
\begin{gathered}
\tau<\tau_{c} \Rightarrow \mu=\rightarrow \infty(\text { solid regime }) \\
\tau>\tau_{c} \Rightarrow \mu=\mu_{c}\left(\frac{\dot{\gamma}}{\dot{\gamma}_{c}}\right)^{(m-1)}(\text { liquid regime })
\end{gathered}
$$

where $\mu_{c}$ and $\dot{\gamma}_{c}$ are the corresponding critical viscosity and shear rate, respectively. However, this model doesn't describe the aspect of the transient behavior of the material including the elastic and viscous properties in the solid regime. For a paste in the solid regime, the behavior can be represented by a solid viscoelastic model,

$$
\gamma<\gamma_{c}, \tau=G \gamma+\bar{\mu} \dot{\gamma}
$$

where $G$ is the elastic modulus and $\bar{\mu}$ is the viscosity of the paste in the solid regime. The solid-liquid transition occurs at above a critical stress which is the described as the yield stress. For an approximation, the yield stress, defined as the minimum stress required to reach $\gamma_{c}$, 


$$
\tau_{c}=G \gamma_{c}
$$

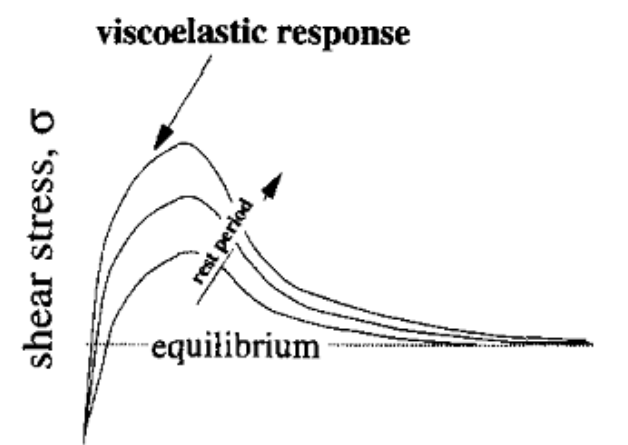

shearing time, $\mathrm{t}$

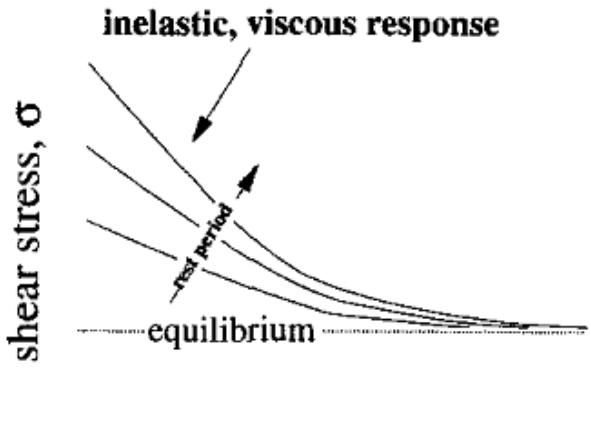

shearing time, $\mathrm{t}$

Figure A.2: Different kinds of thixotropic behavior on startup of shear after various degrees of rest [96].

Coussot et al. [118] used a simple toy model ( $\lambda$--model) based on the thixotropic behavior which is the phenomena of reversible ageing and shear rejuvenation.

$$
\eta=\eta_{0}(1+\beta \lambda)^{n}
$$

and

$$
\frac{d \lambda}{d t}=\frac{1}{\tau}-\alpha \dot{\gamma} \lambda
$$

where $\beta$ and $n$ are parameters indicating how strongly the microstructure influence the viscosity. $\eta_{0}$ is the limiting viscosity at high shear rates and $\tau$ is the characteristic ageing time for building up of the microstructure, $\alpha$ is the rate at which the microstructure is broken down under shear. The figure of merit of this model is that when $d \lambda / d t$ is not zero, it can quantitively describe avalanche behavior and viscosity bifurcation. 